\title{
Bose-Einstein Condensation of Trapped Atomic Gases
}

\author{
Ph.W. Courteille*, V.S. Bagnato*, V.I. Yukalov*,** \\ * Instituto de Física de São Carlos, Universidade de São Paulo, \\ P.O. Box 369, São Carlos, SP 13560-250, Brazil \\ ** Bogolubov Laboratory of Theoretical Physics, \\ Joint Institute for Nuclear Research, Dubna 141980, Russia \\ e-mail: yukalov@thsun1.jinr.ru
}

February 1, 2008 


\begin{abstract}
This article reviews recent investigations on the phenomenon of Bose-Einstein condensation of dilute gases. Since the experimental observation of quantum degeneracy in atomic gases, the research activity in the field of coherent matter-waves literally exploded. The present topical review aims to give an introduction into the thermodynamics of Bose-Einstein condensation, a general overview over experimental techniques and investigations, and a theoretical foundation for the description of bosonic many-body quantum systems.
\end{abstract}




\section{Contents}

1 Introduction 1

2 Basic Notions $\mathbf{5}$

2.1 Bose-Einstein Condensation of Ideal Gas . . . . . . . . . . . . . . . . . . . . . . . . . . . . . . . 6

2.2 Thermodynamics of Ideal Confined Bose-Gas . . . . . . . . . . . . . . . . . . . . . . . . . . . . . 7

2.3 Low-Dimensional Systems . . . . . . . . . . . . . . . . . . . . . . . . . . . . . . . . . . 9

2.4 Semiclassical Density Distribution . . . . . . . . . . . . . . . . . . . . . . . . . 10

2.5 Finite Number of Particles . . . . . . . . . . . . . . . . . . . . . . . . . . . . . . . . 11

2.6 Atomic Interactions in Nonideal Confined Bose-Gas . . . . . . . . . . . . . . . . . . . . . . . . . 12

2.6 .1 Semiclassical Approximation . . . . . . . . . . . . . . . . . . . . . . . . 13

2.6 .2 Attractive Interactions . . . . . . . . . . . . . . . . . . . . . . . . . . . . . . 14

2.7 Classification of Phase Transitions . . . . . . . . . . . . . . . . . . . . . . . . . . . . . . . . . . 14

3 Making and Probing Bose-Einstein Condensates 17

3.1 Techniques for Cooling and Trapping . . . . . . . . . . . . . . . . . . . . . . . . . . 18

3.1.1 Magneto-Optical Traps . . . . . . . . . . . . . . . . . . . . . . . . . . . . . 19

3.1.2 $\quad$ Far-off Resonance Dipole Traps . . . . . . . . . . . . . . . . . . . . . . . . . . . . . . . . . 19

3.1.3 Magnetic Traps . . . . . . . . . . . . . . . . . . . . . . . . . . . . . . . . . . . 20

3.1.4 Evaporative Cooling . . . . . . . . . . . . . . . . . . . . . . . . . . . . 21

3.2 Realization of Bose-Einstein Condensation . . . . . . . . . . . . . . . . . . . . . . . . . . . . . . . 22

3.2.1 BEC in Alkalis . . . . . . . . . . . . . . . . . . . . . . . . . . . . 23

3.2 .2 BEC in Hydrogen . . . . . . . . . . . . . . . . . . . . . . . . . . . . . . . . . 24

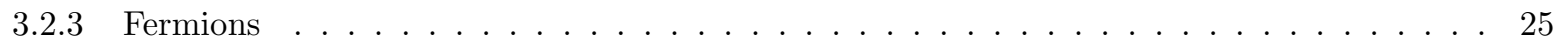

3.3 Imaging Techniques . . . . . . . . . . . . . . . . . . . . . . . . . 25

3.3.1 Time-of-Flight Imaging . . . . . . . . . . . . . . . . . . . . . . . . . . . . . 26

3.3.2 In-Situ Imaging . . . . . . . . . . . . . . . . . . . . . . . . . . . . . . 27

3.4 Measurements on Condensate Equilibrium Thermodynamics . . . . . . . . . . . . . . . . . . . . . 28

4 Experiments on Condensate Dynamics 29

4.1 Wavepacket Dynamic . . . . . . . . . . . . . . . . . . . . . . . . . . . . . . . 29

4.2 Multicomponent Condensates . . . . . . . . . . . . . . . . . . . . . . . . . . . . . . . . 29

$4.2 .1 \quad$ Double Species Condensates in ${ }^{87} \mathrm{Rb}$. . . . . . . . . . . . . . . . . . . . . . . . . . . 29

4.2 .2 Spinor Condensates in ${ }^{23} \mathrm{Na}$. . . . . . . . . . . . . . . . . . . . . . . . 30

4.3 Collective Excitations . . . . . . . . . . . . . . . . . . . . . . . . . . 31

4.3.1 Elementary Excitations . . . . . . . . . . . . . . . . . . . . . . . 31

4.3 .2 Non-Circulating Topological Modes . . . . . . . . . . . . . . . . . . . . . . . . . . . . . . . 32

4.3 .3 Superfluid Flow . . . . . . . . . . . . . . . . . . . . . . . . . . . 32

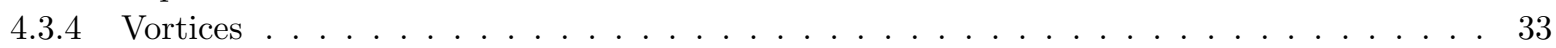

4.3 .5 Matter-Wave Solitons . . . . . . . . . . . . . . . . . . . . . . . 36 
\begin{tabular}{|ll}
5 Atom Optics with Bose-Einstein Condensates & 39
\end{tabular}

5.1 Conventional Atom Optics . . . . . . . . . . . . . . . . . . . . . . . . . . . 40

5.1 .1 Atom Optical Devices . . . . . . . . . . . . . . . . . . . . . . . . 40

5.1 .2 Atom Interferometers . . . . . . . . . . . . . . . . . . . . . . . . 41

5.2 Atom Laser . . . . . . . . . . . . . . . . . . . . . . . . . . . 4 41

5.2 .1 Bosonic Stimulation and Evaporation . . . . . . . . . . . . . . . . . . . . . . . . . . . . . 42

5.2 .2 Coherence and Interference . . . . . . . . . . . . . . . . . . . . . . . . . . . . . . . . 42

5.2 .3 Output Coupling . . . . . . . . . . . . . . . . . . . . . . . . 43

5.3 Atom Interferometry . . . . . . . . . . . . . . . . . . . . . . . . . . . . . . . . . . 44

$5.3 .1 \quad$ Double Species Interferometer and Phase Measurements . . . . . . . . . . . . . . . . . . . 44

5.3 .2 Quantum Transport and Josephson Tunneling . . . . . . . . . . . . . . . . . . . . . . . . . 45

5.3 .3 Bragg Diffraction . . . . . . . . . . . . . . . . . . . . . . . . 46

5.4 Nonlinear Atom Optics . . . . . . . . . . . . . . . . . . . . . . . . . . . . . 47

5.4 .1 Self-Defocussing . . . . . . . . . . . . . . . . . . . . . . . . 48

5.4 .2 Dispersion . . . . . . . . . . . . . . . . . . . . . . . . . . . . . 48

5.4 .3 Second Harmonic Generation . . . . . . . . . . . . . . . . . . . . . . . . . . . . . . . . . . 49

5.4 .4 Four-Wave Mixing and Phase Conjugation. . . . . . . . . . . . . . . . . . . . . . . . . 50

5.4 .5 Spin Mixing . . . . . . . . . . . . . . . . . . . . . . 51

5.4 .6 Dielectric Properties of Bose-Einstein Condensates . . . . . . . . . . . . . . . . . . . . . . 51

5.5 Coherent Coupling of Optical Fields and Matter-Waves . . . . . . . . . . . . . . . . . . . . . . . 52

5.5 .1 Superradiant Rayleigh Scattering . . . . . . . . . . . . . . . . . . . . . . . . . 52

5.5 .2 Matter-Wave and Light Amplification . . . . . . . . . . . . . . . . . . . . . . . . . . . . 54

5.5 .3 Quantum Optics with Atoms . . . . . . . . . . . . . . . . . . . . . . 55

6 Collision Resonances 5

6.1 Feshbach Resonances in ${ }^{85} \mathrm{Rb}$ and ${ }^{23} \mathrm{Na}$. . . . . . . . . . . . . . . . . . . . . . . . . . 57

6.2 Bose-Einstein Condensation in ${ }^{85} \mathrm{Rb}$. . . . . . . . . . . . . . . . . . . . . . . . . . . . . . . 58

6.3 Molecular Bose-Einstein Condensates . . . . . . . . . . . . . . . . . . . . . . . . . . . 58

7 Criteria of Bose-Einstein Condensation 61

7.1 Einstein Criterion of Condensation . . . . . . . . . . . . . . . . . . . . . . . . . . . . . 61

7.2 Penrose Criterion of Condensation . . . . . . . . . . . . . . . . . . . . . . . . . . . 63

7.3 Off-Diagonal Long-Range Order . . . . . . . . . . . . . . . . . . . . . . . . . . . . . . . . . . . . 64

7.4 Broken Gauge Symmetry $\ldots$. . . . . . . . . . . . . . . . . . . . . . . . . . . . . . . . . . . . 64

7.5 Condensation in Confined Systems . . . . . . . . . . . . . . . . . . . . . . . . . . . . . . . . . . 66

8 Coherent Atomic States 69

8.1 Definition and Main Properties . . . . . . . . . . . . . . . . . . . . . . . . . . . . . 69

8.2 Stationary Coherent States . . . . . . . . . . . . . . . . . . . . . . . . . . . . . . . . . 71

8.3 Quantum Coherent Averages . . . . . . . . . . . . . . . . . . . . . . . . . . 73

8.4 Statistical Coherent Averages . . . . . . . . . . . . . . . . . . . . . . . . . . . . . . . 75

8.5 Correlation Functions and Coherence . . . . . . . . . . . . . . . . . . . . . . . . . . . . . . . . 76

9 Meaning of Gross-Pitaevskii Equation 79

9.1 Coherent Wave Function . . . . . . . . . . . . . . . . . . . . . . . . . . . . . . . . 79

9.2 Condensate Order Parameter . . . . . . . . . . . . . . . . . . . . . . . . . . . . . . . . 80

9.3 General Anisotropic Case . . . . . . . . . . . . . . . . . . . . . . . . . . . . . . . . . . 81

9.4 Cylindrically Symmetric Trap . . . . . . . . . . . . . . . . . . . . . . . . . . . . . . . . . . . . 82

9.5 Thomas-Fermi Ground State . . . . . . . . . . . . . . . . . . . . . . . . . . 83 


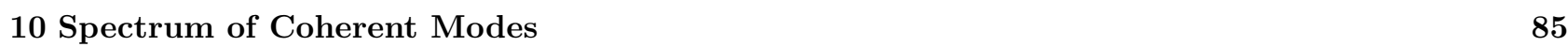

10.1 Optimized Perturbation Theory $\ldots \ldots \ldots \ldots \ldots \ldots$. . . . . . . . . . . . 85

10.2 Isotropic Ground State . . . . . . . . . . . . . . . . . . . . . . . . . 87

10.3 Anisotropic Excited State $\ldots \ldots \ldots$. . . . . . . . . . . . . . . . . . . . . . 89

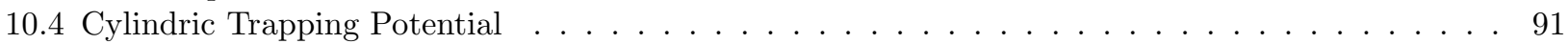

10.5 Cloud Shape and Lifetime $\ldots \ldots \ldots \ldots$. . . . . . . . . . . . . . . . . 94

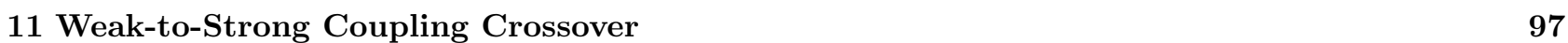

11.1 Self-Similar Crossover Approximants $\ldots \ldots \ldots$. . . . . . . . . . . . . . . . . . . 97

11.2 One-Dimensional Confined System $\ldots \ldots \ldots$. . . . . . . . . . . . . . . . . . . . 99

11.3 Spherically Symmetric Trap . . . . . . . . . . . . . . . . . . . . . . . . . . . . . 101

11.4 Traps of Cylindrical Shapd . . . . . . . . . . . . . . . . . . . . . . . . . . . . . 103

11.5 Strong-Coupling and Thermodynamic Limits . . . . . . . . . . . . . . . . . . . 105

\begin{tabular}{|r|r}
12 Vortices in Trapped Condensates & 109
\end{tabular}

12.1 Vortex Transition Frequencies $\ldots$. . . . . . . . . . . . . . . . . . . . . . . . . . . . . . 109

12.2 Effective Radial Equation $\ldots \ldots \ldots$. . . . . . . . . . . . . . . . . . . 110

12.3 Vortex Wave Function $\ldots \ldots \ldots \ldots$. . . . . . . . . . . . . . . . . . 112

\begin{tabular}{|r|r|}
\hline 13 Elementary Collective Excitations & 117
\end{tabular}

13.1 Linearization of Gross-Pitaevskii Equation . . . . . . . . . . . . . . . . . . . . 117

13.2 Linearization of Hydrodynamic Equations $\ldots \ldots \ldots \ldots \ldots \ldots$

13.3 Lagrangian Variational Technique $\ldots \ldots \ldots \ldots$. . . . . . . . . . . . . . . 120

\begin{tabular}{|r|r}
\hline 14 Multicomponent Bose Mixtures & 123
\end{tabular}

14.1 Coherent States of Mixtures . . . . . . . . . . . . . . . . . . . . . . . . 123

14.2 Branching of Excitation Spectrum . . . . . . . . . . . . . . . . . . . . . 125

14.3 Dynamic and Thermodynamic Stability . . . . . . . . . . . . . . . . . . . 126

14.4 Stratification of Moving Components . . . . . . . . . . . . . . . . . . . . . . . 129

14.5 Mixing by Feshbach Resonance $\ldots \ldots \ldots$. . . . . . . . . . . . . . . . . 131

\begin{tabular}{|r|r|}
15 Topological Coherent Modes & 135
\end{tabular}

15.1 Resonance Field Modulation . . . . . . . . . . . . . . . . . . . . . . . . . 135

15.2 Critical Dynamic Effects . . . . . . . . . . . . . . . . . . . . . . . . . . . . . . . 139

15.3 Spatio-Temporal Evolution of Density $\ldots \ldots \ldots$. . . . . . . . . . . . . . . . 142

15.4 Resonance Formation of Vortices . . . . . . . . . . . . . . . . . . . . . . . . . . 144

15.5 Problems in Resonance Excitation $\ldots \ldots \ldots \ldots$. . . . . . . . . . . . . 145

16 Coherence and Atom Lasers 149

16.1 Interference and Josephson Effect . . . . . . . . . . . . . . . . . . . . . . . . . . 149

16.2 Conditions on Atom Laserd $\ldots \ldots \ldots \ldots$. . . . . . . . . . . . . . . 151

16.3 Nonadiabatic Dynamics of Atoms . . . . . . . . . . . . . . . . . . . . 153

16.4 Scale Separation Approach $\ldots \ldots \ldots \ldots$. . . . . . . . . . . . . . 156

16.5 Magnetic Semiconfinement of Atoms f . . . . . . . . . . . . . . . . . . 158

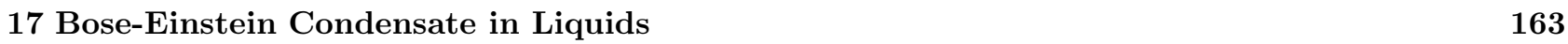

17.1 Differences between Liquids and Gases . . . . . . . . . . . . . . . . . . . . . . . . 163

17.2 Definition of Superfluid Density $\ldots \ldots \ldots \ldots \ldots$. . . . . . . . . . . . . 165

17.3 Spectrum of Collective Excitations $\ldots \ldots \ldots \ldots$. . . . . . . . . . . . 170

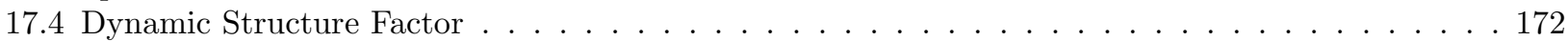

17.5 Measurement of Condensate Fraction $\ldots \ldots \ldots$. . . . . . . . . . . . . . . 174 


\section{Chapter 1}

\section{Introduction}

In classical physics, light is a wave and matter consists of particles. At the beginning of the twentieth century, new experiments like the discovery of the photo effect shattered the common view of life. Those observations could only be explained by the assumption that light consists of quantized energy packets, similar to particles. The feature that light sometimes appears as a wave and sometimes as a particle seemed incompatible. This duality of light was understood within the framework of the newly developed quantum theory which benefitted from important contributions from scientists including Max Planck, Niels Bohr, Werner Heisenberg and Albert Einstein. Together with Einstein's theory of relativity the quantum theory today constitutes the fundamental pillar of modern physics. Louis de Broglie applied the duality principle also to material particles. According to him, very cold particles should under certain conditions behave like waves whose wavelengths increase as their velocity drops. The particle is delocalized over a distance corresponding to the de Broglie wavelength. These features were soon discovered experimentally and are today even used commercially, e.g. in electron microscopes.

The laser was discovered in 1956. In a laser, light particles are forced to oscillate synchronously, i.e. coherently. By analogy, we may now raise the question if a similar phenomenon can occur for material particles, and if it should in principle be possible to construct an atom laser. Such a device would emit coherent matterwaves just like the laser emits coherent light. When a gas is cooled down to very low temperatures, the individual atomic de Broglie waves become very long and, if the gas is dense enough, eventually overlap. If the gas consists of a single species of bosonic particles all being in the same quantum state, the de Broglie waves of the individual particles constructively interfere and build up a huge coherent matter-wave. The matter-wave is described by a single quantum mechanical wavefunction exhibiting long range order and having a single phase. If this wavefunction is formed in a trap, all the atoms pile up in its ground state. The transition from a gas of individual atoms to the mesoscopic quantum degenerate many-body state occurs as a phase transition and is named after Bose and Einstein who calculated the effect as early as 1924 [1], 2] Bose-Einstein condensation (BEC).

The vast interest in Bose-Einstein condensation arises partly from the fact that this phenomenon touches several physical disciplines thus creating a link between them: In thermodynamics BEC occurs as a phase transition from gas to a new state of matter, quantummechanics view BEC as a matter-wave coherence arising from overlapping de Broglie waves of the atoms and draw an analogy between conventional and "atom lasers", quantumstatistics explain BEC as more than one atom sharing a phase space cell, in the quantum theory of atomic traps many atoms condense to the ground state of the trap, in quantum field theory BEC is closely related to the phenomenon of spontaneous breaking of the gauge symmetry.

The experimental verification of Bose-Einstein condensation has been a long cherished dream in physics. On one hand, several phenomena have been related to BEC in the past, e.g. the phenomenon of superfluidity in liquid helium and the superconductivity. On the other hand, those strongly interacting systems are not pure enough to clearly identify the role of the Bose-condensation. A few years ago, however, Bose-Einstein condensation in weakly interacting confined atomic gases was achieved in experiments [3]- 6]. The observation of Bose-Einstein condensation has now been confirmed by more than twenty groups worldwide and triggered 
an enormous amount of theoretical and experimental work on the characterization of Bose-condensed gases. While the early work focussed on the equilibrium thermodynamics of condensates close to the phase transition, very soon the dynamical response of the condensate wavefunction to perturbations was subject of thorough investigations. Subsequently, the general attention turned to the study of the superfluid characteristics of BECs, phenomena of quantum transport and the interaction of BECs with light. Meanwhile, exotic states like multiple species condensates [7, 8] and vortices [9, 10] have been created, Feshbach collision resonances have been found [11]-13] various kinds of atom lasers have been constructed [14]- 18] BEC interferometers have been realized [19], diffraction experiments have been carried out with BECs [20], nonlinear matter-wave interactions [21] and matter-wave amplification [22]-224] have been observed.

One of the most exciting features is the possibility to construct atom lasers. The technical advances made in the past few years in controlling and manipulating matter-waves have raised a new field called atom optics. Nearly all optical elements which are used to manipulate light beams have found their atom-optical counterpart within the past ten years, including mirrors, lenses, waveguides, acousto-optical modulators, and so on. The occurrence of large-scale coherent quantum objects like BECs and atom lasers will definitively lead to a modernization of the fields of atomic interferometry, holography, lithography and microscopy. Collisions between atoms add a rich variety of phenomena to the field of coherent matter-wave optics where they play a role similar to the role played by atom-photon interactions in quantum and nonlinear optics. Since the experimental observation of matter-wave four-wave mixing [21] the field of nonlinear matter-wave optics [25] is evolving at very high speed.

The characteristics (shape, stability, quantum depletion,...) and the dynamics (superfluidity, nonlinear excitations,...) of BECs are largely governed by interactions between the atoms. The importance of atomic collisions for BEC turns them into interesting subject for studies. Low-energy scattering phenomena, like the recently found Feshbach collision resonances [11, 12] may be used to coherently couple a bound state of two atoms to the unbound continuum [26]. This is particularly interesting for the development of techniques capable of producing ultracold molecules right inside a trap (ultracold chemical engineering), or even to produce molecular BECs.

Finally, the field of atomic quantum optics is being launched with many interesting theoretical predictions and ideas. Atomic quantum optics could be defined as the matter-wave counterpart of quantum optics with light fields. In analogy, one might expect the possibility of building up "nonclassical" quantum correlations, e.g. Schrödinger cat like quantum states in a truly mesoscopic quantum system (expanded BEC wavefunctions may easily range up to millimeter sizes) [27]. Those states have been studied in various quantum optical systems. But even more important is the possibility of coherently coupling the optical, motional, and internal degrees of freedom and therefore the entanglement of the related modes. In such systems, quantum optics of laser modes (Cavity QED) and matter-wave optics will merge. There are already several ideas about the implementation of mutual coherent quantum control between optical and matter-wave modes [28], and an ultracold version of the Correlated Atomic Recoil Laser (CARL), an atomic analogue of the Free-Electron Laser (FEL), may play the role of an interface between optical and matter-wave fields [29].

This topical review is organized as follows. The introduction into the basic notions of the thermodynamics of Bose-Einstein condensation (Chapter 2) is brief, since many excellent papers and textbooks have been published on this subject. Chapter 3 reviews experimental approaches to BEC and points out the essential techniques to achieve and probe condensates. These have been covered by several review articles as well, so that we just give a short overview. Ever since the first achievement of BEC in a dilute gas, the experimental progress has been very fast. Almost every month a new milestone-experiment is published and any attempt of writing a review is hence outdated at the time of publication. Nevertheless, we believe that a review of the recent experiments is helpful to show the state of the art in BEC manipulation and to point out the challenges which still lie ahead. We devoted Chapters 6 , 5 and 6 to this subject.

There are two good reviews discussing the physics of trapped Bose-Einstein condensates [30, 31. The theoretical part of our review differs from the latter in several aspects. First of all, we thoroughly investigate those principal notions, whose discussion is rarely met in literature but which are crucial to answer such basic questions as: What is Bose-Einstein condensation actually and what are the mathematically correct criteria for this phenomenon? What is the relation of Bose condensation to coherence and to gauge symmetry breaking? What is the true meaning of the famous Gross-Pitaevskii equation? Is it possible to produce non-groundstate 
condensates?

Trapped atoms compose a nonlinear nonuniform system, whose description is essentially more complicated than that of uniform systems. We explain in more details than usually done mathematical techniques helping to treat such nonlinear and nonuniform problems. This especially concerns those original methods that have been developed recently and which cannot be found in other reviews.

Our paper contains some fresh topics that have not been reviewed earlier. Among these are: the stratification of condensate components moving with respect to each other; resonance formation of topological coherent modes and critical effects that can arise during this resonance process; non-adiabatic dynamics of trapped atoms and their escape from a trap.

Finally, in Chapter 17, the problems of describing and measuring Bose-Einstein condensate in quantum liquids, such as superfluid helium, are discussed. This makes it possible to better understand analogies and differences between liquids and gases. 


\section{Chapter 2}

\section{Basic Notions}

The canonical approach to statistical mechanics starts with Boltzmann's probabilistic analysis of the velocity distribution of an ideal gas. For a gas composed of particles of mass $m$ at temperature $T$, the velocity distribution is given by the well-known Maxwell-Boltzmann (MB) law [32]

$$
g(\mathbf{v})=\left(\frac{m}{\sqrt{2 \pi k_{B} T}}\right)^{3} \exp \left(-\frac{m \mathbf{v}^{2}}{2 k_{B} T}\right)
$$

where $k_{B}$ is the Boltzmann constant. The Maxwell-Boltzmann law was first experimentally tested by Otto Stern in 1920 using a primitive atomic beam and a simple time-of-flight technique based on a velocity-selective rotating drum. With the advent of laser spectroscopy, the MB law and its limitations could be tested with highly improved precision. This law describes well the behavior of weakly interacting atoms at high temperatures. Deviations from it are insignificant until quantum mechanical effects assert themselves, and this does not occur until the temperature becomes so low that the atomic de Broglie wavelength becomes comparable to the mean distance between particles. For a gas in equilibrium the characteristic wavelength is

$$
\lambda_{d B}=\sqrt{\frac{2 \pi \hbar^{2}}{m k_{B} T}},
$$

where $\hbar=h / 2 \pi$ is Planck's constant. For a general system with density $n$, the mean distance between particles is $n^{-1 / 3}$. Quantum effects are expected to show up for $n^{-1 / 3} \sim \lambda_{d B}(T)$, so that the boundary to this regime is defined by

$$
k_{B} T(n)=\frac{2 \pi \hbar^{2}}{m} n^{2 / 3} .
$$

For example, an atomic gas at $900 \mathrm{~K}$ and $n \sim 10^{16} \mathrm{~cm}^{-3}$ is safely within the classical regime, since $n^{-1 / 3} \sim$ $10^{6} \mathrm{~cm} \gg \lambda_{d B}=10^{-9} \mathrm{~cm}$. To witness quantum effects one needs atoms at low temperature and relatively high density. For most gases, lowering the temperature or increasing density promotes the system to liquidity before the quantum regime is reached. Well-known exceptions are spin-polarized hydrogen $(\mathrm{H} \uparrow)$ which does not get liquid at all and helium which exhibits effects of quantum degeneracy in the liquid phase, although those effects are rather complex due to strong interparticle forces.

All particles of the quantum world are either bosons with integer spin or fermions with half-integer spin. Fermions do not share a quantum state, because they must follow Pauli's exclusion principle. They obey a quantum statistical distribution called Fermi-Dirac distribution. In contrast, bosons enjoy sharing a quantum state and even encourage other bosons to join them in a process called bosonic stimulation. Bosons follow a quantum statistical distribution called Bose-Einstein distribution (BE). In this article, we will mainly focus on the Bose-Einstein distribution. The basic difference between MB statistics and BE statistics is that the former applies to identical particles that nevertheless are distinguishable from one another, while the latter describes identical indistinguishable particles. For Bose-Einstein statistics, one can derive [33] the Bose-Einstein 
distributed occupation number for a non-degenerate quantum state at energy $\varepsilon$ when the system is held at temperature $T$,

$$
f(\varepsilon)=\frac{1}{e^{\beta(\varepsilon-\mu)}-1},
$$

where we used the short-hand notation $\beta \equiv 1 / k_{B} T$. The chemical potential $\mu$ is an important parameter of the system, which helps normalizing the distribution $f(\varepsilon)$ to the total number of particles,

$$
N=\sum_{\varepsilon} f(\varepsilon) .
$$

Similarly, the total energy of the system is given by

$$
E=\sum_{\varepsilon} \varepsilon f(\varepsilon)
$$

A very remarkable effect occurs in a bosonic gas at a certain characteristic temperature: below this temperature a substantial fraction of the total number of particles occupies the lowest energy state, while each of the remaining states is occupied by a negligeable number of particles. Above the transition temperature the macroscopic observables of the gas, like pressure, heat capacity, etc., receive contributions from all states with a certain statistical weight but without favouring the lowest energy state. Below the transition temperature, the observables are altered by the macroscopic occupation of the ground state, which results in dramatic changes in the thermodynamic properties. The phase transition is named after Shandrasekar Bose [] and Albert Einstein [2] Bose-Einstein Condensation (BEC).

\subsection{Bose-Einstein Condensation of Ideal Gas}

One of the keys to understand BEC is the behaviour of the chemical potential $\mu$ at very low temperatures. The chemical potential is responsible for the stabilization of the large number of atoms in the ground state $N_{0}$. A system of a large number $N$ of noninteracting bosons condenses to the ground-state as the temperature goes to zero, $N_{0} \rightarrow N$. The Bose-Einstein distribution function (2.4) gives the ground state population, $\varepsilon=0$, in the zero-temperature limit, $N=\lim _{T \rightarrow 0}\left(e^{-\beta \mu}-1\right)^{-1}=-1 / \beta \mu$, or in terms of the fugacity $Z=e^{\beta \mu}$,

$$
Z \sim 1-1 / N
$$

Note that the chemical potential in a bosonic system must always be lower than the ground-state energy, in order to guarantee non-negative occupancy $f(\varepsilon)$ of any state. $Z \sim 1$ denotes macroscopic occupation of the ground state. We define the critical temperature for Bose-Einstein condensation via the occupancy of the ground state. Above this temperature the occupancy of the ground state is not macroscopic, below this point it is.

For a noninteracting Bose gas with $N$ particles of mass $m$ confined in a hard-wall box of volume $V=L^{3}$ the critical temperature for BEC can be calculated by equation (2.3). The boundary conditions require that the momenta satisfy $p_{j}=2 \pi \hbar l_{j} / L$, where $j=x, y$ or $z$ and $l_{j}$ are integers. Each state is labeled by a set of three integers $\left(l_{x}, l_{y}, l_{z}\right)$. In the thermodynamic limit, the sum over all quantum states may be converted to an integral over a continuum of states,

$$
\sum_{\mathbf{p}} \stackrel{N \rightarrow \infty}{\longrightarrow} \frac{V}{h^{3}} \int d^{3} \mathbf{p}
$$

For a free gas with energy $\varepsilon=p^{2} / 2 m$, we can derive the density of states $\rho(\varepsilon)$ from the normalization of the phase space,

$$
\begin{aligned}
1 & =h^{-3} \iint d^{3} \mathbf{r} d^{3} \mathbf{p} \\
& =2 \pi \sqrt{2 m}^{3} \frac{V}{h^{3}} \int_{0}^{\infty} \sqrt{\varepsilon} d \varepsilon \equiv \int_{0}^{\infty} \rho(\varepsilon) d \varepsilon .
\end{aligned}
$$

The density of states basically depends on the geometry of our system. For a homogeneous system we find $\rho(\varepsilon)=2 \pi \sqrt{2 m}^{3} V / h^{3} \sqrt{\varepsilon}$, but we can easily extend this result to inhomogeneous systems (Section 2.2). We 
should, however, keep in mind that the density-of-states approach is an approximation which is not valid for experiments with limited numbers of atoms (Section 2.5). Using the occupation number $f(\varepsilon)$ for the BoseEinstein distribution (2.4), in the thermodynamic limit, we calculate the total number of particles,

$$
\begin{aligned}
N & =N_{0}+h^{-3} \iint f(\varepsilon(\mathbf{r}, \mathbf{p})) d^{3} \mathbf{r} d^{3} \mathbf{p} \\
& =N_{0}+\int_{0}^{\infty} f(\varepsilon) \rho(\varepsilon) d \varepsilon=N_{0}+2 \pi \sqrt{2 m}^{3} \frac{V}{h^{3}} \int_{0}^{\infty} \frac{\varepsilon^{1 / 2} d \varepsilon}{e^{\beta(\varepsilon-\mu)}-1},
\end{aligned}
$$

where the ground state population $N_{0}$ is explicitly retained. In the process of converting the sum into an integral (2.8) the density of states goes to zero approaching the ground state. This error is corrected by adding a contribution $N_{0}$ to the integral. At this point, we introduce the Bose-function that will help to simplify the notation by

and its integral representation

$$
g_{\eta}(z)=\sum_{t=1}^{\infty} \frac{z^{t}}{t^{\eta}}
$$

$$
g_{\eta}(z)=\frac{z r^{\eta}}{\Gamma(\eta)} \int_{0}^{\infty} \frac{x^{\eta-1} d x}{e^{r x}-z}
$$

where $\Gamma(\eta)$ denotes the Gamma function. With this definition, equation (2.10) reads

$$
N=N_{0}+\frac{V}{\lambda_{d B}^{3}(T)} g_{3 / 2}\left(e^{\beta \mu}\right) .
$$

We can use equation (2.13) to calculate the critical temperature $T_{c}^{0}$, defined through $N_{0} \rightarrow 0$ and $\mu \rightarrow 0$. Above the phase transition, $T>T_{c}^{0}$, the population is distributed over all the states, each state being weakly occupied. Below $T_{c}^{0}$ the chemical potential is "pinned" at $\mu=0$ and the number of particles occupying the excited states is

$$
N_{\text {therm }}=\frac{V}{\lambda_{d B}(T)^{3 / 2}} g_{3 / 2}(1)=N\left(\frac{T}{T_{c}^{0}}\right)^{3 / 2}
$$

with $g_{3 / 2}(1)=2.612$. Since $N_{0}+N_{\text {therm }}=N$, the number of particles in the ground state becomes

$$
\frac{N_{0}}{N}=1-\left(\frac{T}{T_{c}^{0}}\right)^{3 / 2}
$$

which is the fraction of the atomic cloud being condensed in the ground state. The abrupt occurrence of a finite occupation in a single quantum state at $T_{c}^{0}$ indicates a spontaneous change in the system and a thermodynamic phase transition. We will come back to this in Section 2.7 .

\subsection{Thermodynamics of Ideal Confined Bose-Gas}

If the atoms are confined in a spatially varying potential, the critical temperature $T_{c}^{0}$ can be significantly altered. The critical temperature depends on the general shape and on the steepness of the potential. We consider $N$ particles of an ideal Bose-gas distributed over various quantum states of an arbitrary potential. The occupation number $f(\varepsilon)$ of particles in an energy level $\varepsilon$ is still given by (2.4), the ground state energy is set to zero. In the thermodynamic limit, the relation between the chemical potential and the total number of particles is given by generalization of equation (2.10), with the appropriate density of states $\rho(\varepsilon)$. The density of state for an arbitrary confining potential $U(\mathbf{r})$ can be found by a generalization of the calculation for the free gas. The volume in phase space between the surfaces of energy $\varepsilon$ and $\varepsilon+d \varepsilon$ is proportional to the number of states in that energy interval. However, the external potential limits the space available to the gas. The density of states is calculated in analogy to equation (2.9) and yields 34, 35.

$$
\rho(\varepsilon)=2 \pi \sqrt{2 m}^{3} \frac{1}{h^{3}} \int_{V^{*}(\varepsilon)} \sqrt{\varepsilon-U(\mathbf{r})} d^{3} \mathbf{r}
$$


where $V^{*}(\varepsilon)$ is the available space for particles with energy $\varepsilon$. We assume a generic power-law potential confining an ideal Bose-gas in $\alpha$ dimensions,

$$
U(\mathbf{r})=\sum_{i=1}^{\alpha}\left|\frac{x_{i}}{a_{i}}\right|^{t_{i}}
$$

and define a parameter describing the confinement power of the potential,

$$
\eta=\frac{\alpha}{2}+\sum_{i=1}^{\alpha} \frac{1}{t_{i}}
$$

Although the temperature is the basic thermodynamical state variable, the system needs to be characterized by other variables. Heat is not a state variable, because the amount of heat required to raise the temperature of the system depends on the way the heat is transferred. The heat capacity quantifies the ability of the system to retain energy. In conventional systems, the heat capacity is typically either given at constant volume or at constant pressure. With this specification heat capacities are extensive state variables. When crossing a phase transition, the temperature dependence of the heat capacity measures the degree of changes in the system above and below the critical temperature and provides valuable informations about the general type of phase transitions.

The total energy of the system is given by:

$$
E(T)=\int_{0}^{\infty} \varepsilon f(\varepsilon) \rho(\varepsilon) d \varepsilon .
$$

For a confined gas, volume and temperature are connected, and the concept of pressure is somewhat vague. In this case we cannot refer to heat capacity at constant volume or constant pressure. However, we may define the heat capacity at a fixed number of particles,

$$
C(T)=\frac{\partial E(T)}{\partial T}
$$

Keeping the implicit temperature dependencies of the thermodynamic variables in mind, we can evaluate (2.19):

$$
C(T)=\beta \int_{0}^{\infty} \varepsilon f(\varepsilon)^{2} \rho(\varepsilon)\left[\mu^{\prime}(T)+\frac{\varepsilon-\mu}{T}\right] e^{\beta(\varepsilon-\mu)} d \varepsilon,
$$

where the derivative of the chemical potential from above $T \rightarrow T_{c}^{0}$ is

$$
\mu^{\prime}\left(T_{c}^{+}\right)=-\frac{1}{T} \frac{\int_{0}^{\infty} \varepsilon f(\varepsilon)^{2} \rho(\varepsilon) e^{\beta \varepsilon} d \varepsilon}{\int_{0}^{\infty} f(\varepsilon)^{2} \rho(\varepsilon) e^{\beta \varepsilon} d \varepsilon} .
$$

It is especially interesting to compare the discontinuity of the heat capacity and of its derivative $\partial C(T) / \partial T$ for various potential power laws and dimensions of confinement, since this may clarify the nature of the phase transition. The thermodynamic quantities take a particularly simple form for power law potentials. The calculations are analogous to those carried out for homogeneous Bose-gases (last section), and we restrict ourselves here to giving the general results for the thermodynamic quantities [35, 36], e.g. internal energy $E$ and heat capacity $C$ :

$$
\begin{aligned}
\frac{N_{0}}{N} & =1-\left(\frac{T}{T_{c}}\right)^{\eta} \frac{g_{\eta}(Z)}{g_{\eta}(1)} \\
\frac{E}{N k_{B} T} & =\eta \frac{g_{\eta+1}(Z)}{g_{\eta}(Z)} \\
\frac{C_{T>T_{c}}}{N k_{B}} & =\eta(\eta+1) \frac{g_{\eta+1}(Z)}{g_{\eta}(Z)}-\eta^{2} \frac{g_{\eta}(Z)}{g_{\eta-1}(Z)} \quad, \quad \frac{C_{T<T_{c}}}{N k_{B}}=\eta(\eta+1) \frac{g_{\eta+1}(1)}{g_{\eta}(1)} \\
\frac{\Delta C_{T_{c}}}{N k_{B}} & =\frac{C_{T_{c}^{-}}-C_{T_{c}^{+}}}{N k_{B}}=\xi^{2} \frac{g_{\eta}(1)}{g_{\eta-1}(1)}
\end{aligned}
$$


The Bose-functions at zero chemical potential are just the familiar Rieman zeta functions, $g_{\eta}(1)=\zeta(\eta)$. The expression for the critical temperature for $N$ particles confined in a generic power-law potential in $\alpha$ dimensions reads

$$
T_{c}^{0}=k_{B}^{-1}\left[\frac{h^{\alpha}}{(2 \pi m)^{\alpha / 2}} \frac{N}{2^{\alpha}} \frac{1}{g_{\eta}(1) \prod_{i=1}^{\alpha} a_{i} \Gamma\left(t_{i}^{-1}+1\right)}\right]^{1 / \eta} .
$$

To evaluate the temperature dependence of the thermodynamic variables, we calculate the fugacity $Z(T)=e^{\beta \mu}$ from the second equation of (2.22) [32]. For $T>T_{c}$ we derive the fugacity as the root of $g_{\eta}(Z)=g_{\eta}(1)\left(T_{c} / T\right)^{\eta}$ and for $T<T_{c}$ the fugacity is simply $Z=1$.

Let us give two examples for three-dimensional confinement, $\alpha=3$. The homogeneous 3D box potential inside a volume $V$ is obtained from the power-law formula by setting $t_{i} \longrightarrow \infty$ so that $\eta \equiv 3 / 2$. Evaluating the density of states (2.15), we find $N=N_{0}+g_{3 / 2}(Z) V / \lambda_{d B}^{3}$.

For an anisotropic harmonic potential, $U(\mathbf{r})=\frac{m}{2} \omega_{x}^{2} x^{2}+\frac{m}{2} \omega_{y}^{2} y^{2}+\frac{m}{2} \omega_{z}^{2} z^{2}$, we have $\eta \equiv 3$. We introduce the geometrically averaged secular frequency $\omega_{\text {trap }} \equiv\left(\omega_{x} \omega_{y} \omega_{z}\right)^{1 / 3}$, and the size of the ground state $a_{\text {trap }}=$ $\sqrt{\hbar / m \omega_{\text {trap }}}$. Evaluating the density of states (2.15), we find $N=N_{0}+\left(k_{B} T / \hbar \omega_{\text {trap }}\right)^{3} g_{3}(Z)$. The values for confinement power, critical temperatures, heat capacity and its discontinuity at the phase transition for several potential configurations are shown in Figure 1 and summarized in Table 1 .

Table 1 shows that steeper potential wells (i.e. smaller $a, b$, and $c$ ) give higher values for $T_{c}^{0}$. The critical temperature also depends on the confinement power of the potential

$$
\eta=-\frac{T_{c}^{0}}{N}\left(\frac{d N_{0}}{d T}\right)_{T=T_{c}^{0}}
$$

Larger values of the confinement power result in higher $T_{c}^{0}$. A strongly confining potential can lead to quantum degeneration at much higher critical temperatures and greatly facilitate experimental efforts to achieve BEC. At a given temperature, a strongly confining potential reduces the minimum number of trapped particles required for condensation.

It is also interesting to note, that from the values presented in Table 1, the changes in heat capacity at the phase transition are larger for any power-law potential than for a rigid wall container. This is due to the fact that increasing the energy of the gas requires to work against the confining potential.

\subsection{Low-Dimensional Systems}

The trapping potentials can technically be designed to be very anisotropic, reaching almost two-dimensional pancake-shaped or one-dimensional needle-shaped configurations. The thermodynamics for such systems can easily be formulated as limiting cases of the general formulae presented in Section 2.2 [36, 37]. We will first discuss a Bose-gas confined in a one-dimensional power law potential, $U(x)=\left(\frac{x}{a}\right)^{t}$. In this case, the confinement power (2.17) reads $\eta=1 / t+1 / 2$. From the general formula for the critical temperature (2.23) we get

$$
k_{B} T_{c}^{1 D}=\left(\frac{h}{(2 \pi m)^{1 / 2}} \frac{N}{2 a} \frac{1}{\Gamma(\eta+1 / 2) g_{\eta}(1)}\right)^{1 / \eta} .
$$

According to the properties of the zeta function, $g_{\eta}(1)=\varsigma(\eta)$ is finite only if $t<2$. Therefore, the onedimensional confined gas will exhibit BEC only if the potential power is less than 2, i.e. only if the external potential is more confining than a parabolic potential.

For a two dimensional power-law potential which is symmetric in both directions, $U(x, y)=\left(\frac{x}{a}\right)^{t}+\left(\frac{y}{a}\right)^{t}$, equation (2.17) reads $\eta=2 / t+1$ and the critical temperature is

$$
k_{B} T_{c}^{2 D}=\left(\frac{h^{2}}{2 \pi m} \frac{N}{4 a^{2}} \frac{1}{\Gamma(\eta / 2+1 / 2)^{2} g_{\eta}(1)}\right)^{1 / \eta} .
$$

Unlike in the $1 D$-case, $g_{\eta}(1)$ remains finite for all positive values of $t$. Consequently, for a confined $2 D$ system, $\mathrm{BEC}$ can in principle occur, except for homogeneous systems where $t \rightarrow \infty$. 


\subsection{Semiclassical Density Distribution}

An effect of the inhomogeneous confining potential is a spatial compression of the cloud during cooling and crossing $T_{c}^{0}$. The behavior of this spatial compression can be and has been used as a signature for the occurrence of BEC (Section 3.2.1). In the following, we will calculate the temperature dependence of the spatial density profile near the critical point. We start with 38

$$
n(\mathbf{r})=\left\{\begin{array}{ccc}
\sum_{\varepsilon=0}^{\infty} f(\varepsilon)\left|\psi_{\varepsilon}(\mathbf{r})\right|^{2} & \text { for } & T>T_{c}^{0} \\
N_{0}\left|\psi_{0}(\mathbf{r})\right|^{2}+\sum_{\varepsilon_{1}}^{\infty} f(\varepsilon)\left|\psi_{\varepsilon}(\mathbf{r})\right|^{2} & \text { for } & T<T_{c}^{0}
\end{array}\right\} .
$$

The temperature dependent occupation numbers $f(\varepsilon)$ and the wavefunctions $\psi_{\varepsilon}(\mathbf{r})$ for all states have to be known. Furthermore, we must know $\mu(T)$, which is an important parameter for determining the occupation number $f(\varepsilon)$ for $T>T_{c}^{0}$. Therefore, equation (2.27) is quite difficult to evaluate analytically. There is, however, a different way to do this. The number of particles occupying a given phase space cell is

$$
d N=h^{-3} f(\varepsilon) d^{3} \mathbf{p} d^{3} \mathbf{r}
$$

where $f(\varepsilon)$ is

$$
f(\varepsilon(\mathbf{r}, \mathbf{p}))=\frac{1}{e^{\beta\left(\mathbf{p}^{2} / 2 m+U(\mathbf{r})-\mu\right)}-1} .
$$

The total density of the normal fraction in position space is found by integrating over momentum space

$$
n_{t h}(\mathbf{r})=h^{-3} \int f(\varepsilon) d^{3} \mathbf{p}=\lambda_{d B}^{-3} g_{3 / 2}\left[e^{\beta(\mu-U(\mathbf{r}))}\right]
$$

where we make use of the integral representation of the Bose-function. This formula holds for any trapping potential. If we may now for simplicity assume a harmonic oscillator, $U(\mathbf{r})=\frac{m}{2} \omega_{x}^{2} x^{2}+\frac{m}{2} \omega_{y}^{2} y^{2}+\frac{m}{2} \omega_{z}^{2} z^{2}$, we can similarly calculate the momentum distribution by integrating over position space

$$
\tilde{n}_{t h}(\mathbf{p})=h^{-3} \int f(\varepsilon) d^{3} \mathbf{r}=\hbar^{-3} \lambda_{d B}^{-3} a_{t r a p}^{6} g_{3 / 2}\left[e^{\beta\left(\mu-\mathbf{p}^{2} / 2 m\right)}\right]
$$

where $a_{\text {trap }}$ is the size of the ground state of the harmonic trap. Of course, by integrating the distributions (2.30) and (2.31) we recover the normalization (2.10):

$$
N=\int \tilde{n}_{t h}(\mathbf{p}) d^{3} \mathbf{p}=\int n_{t h}(\mathbf{r}) d^{3} \mathbf{r}
$$

When evaluating (2.30) using a semiclassical approach, we left out the ground-state contribution, which is in fact negligeable above the phase transition $T_{c}^{0}$. Below $T_{c}^{0}$, the contribution of the ground-state to the density (2.30) is given by $N_{0}\left|\psi_{0}\right|^{2}$, where $\psi_{0}$ describes the ground state of the trap. If we assume a harmonic oscillator potential $U(\mathbf{r})=\frac{m}{2} \omega^{2} \mathbf{r}^{2}$, we expect a Gaussian distribution for the ground state density

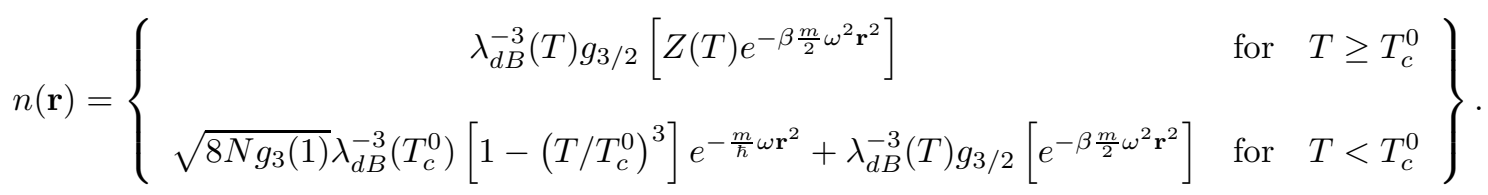

To obtain the evolution of $n(\mathbf{r})$ while the system is cooled down across the phase transition, it is necessary to know the fugacity $Z$ as a function of temperature. We can either numerically solve the second equation of (2.22) separately above and below the phase transition, or we can approximate the fugacity by a series as described below. Above $T_{c}^{0}$ the equation (2.5) can be written

$$
N=\sum_{\varepsilon} \frac{Z \mathbf{e}^{-\beta \varepsilon}}{1-Z \mathbf{e}^{-\beta \varepsilon}}=\sum_{\varepsilon} \sum_{j=1}^{\infty} Z^{j} \mathbf{e}^{-j \beta \varepsilon} .
$$


The sum can be transformed into an integral in the continuum-of-states approximation:

$$
N=\sum_{j=1}^{\infty} D_{j} Z^{j}
$$

where $D_{j}=\int_{0}^{\infty} \rho(\varepsilon) \mathbf{e}^{-j \beta \varepsilon} d \varepsilon$. This series relates the number of particles with the fugacity, where the coefficients $D_{j}$ carry all information about the external potential. The series can be inverted yielding values for the fugacity $Z$. For the harmonic oscillator, we obtain the explicit expression

$$
Z=1.200\left(\frac{T_{c}^{0}}{T}\right)^{3}-0.180\left(\frac{T_{c}^{0}}{T}\right)^{6}-0.010\left(\frac{T_{c}^{0}}{T}\right)^{9}+\text { higher orders. }
$$

We can now evaluate (2.33) at any temperature. For simplicity, we consider the density at $\mathbf{r}=0$. As a function of temperature, the peak density $n(0)$ exhibits a sudden jump, proportional to $N^{1 / 2}$, at the critical temperature (Fig. 2). This behavior is used as an experimental indication for the occurrence of BEC [3]. Larger total particle numbers $N$ make the identification easier. For small numbers, the density jump decreases and may eventually be washed out by fluctuations in the thermal distribution.

Intuitively, one expects Bose condensation to set on when the mean distance between the particles is approximately $\lambda_{d B}$. Indeed, the density distribution (2.33) takes a value of $n_{c}=\lambda_{d B}^{-3} g_{3 / 2}(1)$ at the critical point irrespective of the nature of the confining potential. The main effects of inhomogeneous trapping is to concentrate the density at a smaller region of space and to facilitate the formation of BEC in this region. The quantity $n(0) \lambda_{d B}^{3}=g_{3 / 2}(Z)$ is often called phase space density of the gas.

\subsection{Finite Number of Particles}

The condensates experimentally produced in alkali gases consisted of relatively small atom numbers between 1000 to $10^{7}$, so that the validity of the thermodynamic approximation and the use of the density-of-states approach has been questioned [39]. Furthermore, the decision whether to use the grand canonical, the canonical or the microcanonical ensemble for calculating the thermodynamic quantities noticeably influences the results. Herzog and Olshanii [40] have shown that for small atom numbers on the order of 100 the canonical and grand canonical statistics lead to predictions on the condensed fraction that differ by up to $10 \%$. On the other hand, they give the same results if the particle numbers are large. Which canonical statistics is more appropriate is not a trivial question and depends on the experimental setup and in particular on the time scale of the measurements. If we look at the sample for short times, the number of condensed atoms will be fixed, and we can assume a canonical ensemble. For longer times, however, the atom number may be an equilibrium parameter depending on the contact of the sample with a reservoir, and the grand canonical statistics is better suited.

Assuming grand canonical ensembles, we will now discuss the impact of finite atom numbers on the properties of a Bose-gas at the condensation threshold and, in particular, on the transition temperature and the heat capacity [39]- [43]. To illustrate this point, we numerically calculate the heat capacity of a Bose-gas confined in a three-dimensional isotropic harmonic trap. The energy eigenvalues $\varepsilon_{m}$ are:

$$
\varepsilon_{m}=m \hbar \omega .
$$

For a three-dimensional trap, we must take the degeneracy $\gamma_{m}$ for the levels into account,

$$
\gamma_{m}=\frac{1}{2}(m+1)(m+2) .
$$

We numerically integrate the expression for the number of atoms

$$
N=\sum_{m=0}^{\infty} \gamma_{m} f\left(\varepsilon_{m}\right),
$$

in order to extract the chemical potential $\mu(T)$ from the occupation number (2.4). We start using a certain limited number of levels $m$ and subsequently add more until the result converges. Knowing $\mu(T)$, we can easily estimate the other thermodynamical quantities. The total energy of the system is

$$
E(T)=\sum_{m=0}^{\infty} \gamma_{m} f\left(\varepsilon_{m}\right) \varepsilon_{m}
$$


and the heat capacity is derived from its definition (2.19) in analogy to the continuum-of-states formula $(2.20)$ 43.,

$$
C(T)=\beta \sum_{m=0}^{\infty} \frac{\gamma_{m} \varepsilon_{m} e^{\beta\left(\varepsilon_{m}-\mu\right)}}{\left[e^{\beta\left(\varepsilon_{m}-\mu\right)}-1\right]^{2}}\left(\beta\left(\varepsilon_{m}-\mu\right)+\frac{\partial \mu}{\partial T}\right),
$$

where

$$
\frac{\partial \mu}{\partial T}=-\frac{1}{T} \frac{\sum_{m=0}^{\infty} \gamma_{m}\left(\varepsilon_{m}-\mu\right) e^{\beta\left(\varepsilon_{m}-\mu\right)} f\left(\varepsilon_{m}\right)^{2}}{\sum_{m=0}^{\infty} \gamma_{m} e^{\beta\left(\varepsilon_{m}-\mu\right)} f\left(\varepsilon_{m}\right)^{2}} .
$$

Figure 3 shows the results of the numerical calculations of the heat capacity for different values of $N$. The critical temperature $T_{c}^{0}$ is defined at this discontinuity. If we define the critical temperature $T_{c}$ to coincide with the maximum heat capacity (where $\partial C / \partial T=0$ ), we find $T_{c} / T_{c}^{0}=0.813,0.898$ and 0.946 for $N=100,10^{3}$ and $10^{4}$, respectively. The lowering of the critical temperature for decreasing numbers of particles is due to the fact that smaller systems have larger available effective volume. In the thermodynamic limit $(N \rightarrow \infty)$ the discontinuity appears very clearly. As $N$ decreases, $C(T)$ gets smoother at the transition and the discontinuity disappears. Strictly speaking, the finite system does not undergo a phase transition. On the other hand, the deviation of the behavior of a large finite number system from the thermodynamic limit is reasonably small to justify talking about phase transition.

Grossmann and Holthaus 39] derived analytic expressions for grand canonical ensembles and harmonic traps with $\tilde{\omega}=\left(\omega_{x} \omega_{y} \omega_{z}\right)^{1 / 3}$ and $\bar{\omega}=\frac{1}{3}\left(\omega_{x}+\omega_{y}+\omega_{z}\right)$. For the condensed fraction and the critical temperature they found:

$$
\begin{aligned}
\frac{N_{0}}{N}=g_{2}(Z) \approx 1 & -\left(\frac{T}{T_{c}^{0}}\right)^{3} \frac{g_{3}(Z)}{g_{3}(1)}-\frac{3 \tilde{\omega}}{2 \bar{\omega} N^{1 / 3}}\left(\frac{T}{T_{c}^{0}}\right)^{2} \frac{g_{2}(Z)}{g_{3}(1)^{2 / 3}} \\
T_{c} & \approx T_{c}^{0}\left(1-\frac{\tilde{\omega}}{2 \bar{\omega} N^{1 / 3}} \frac{g_{2}(1)}{g_{3}(1)^{2 / 3}}\right) .
\end{aligned}
$$

\subsection{Atomic Interactions in Nonideal Confined Bose-Gas}

Until now, we only considered non interacting ideal gases. The thermodynamic behavior of such systems is solely governed by statistics or, at low temperatures, by quantum statistics. Real systems are always affected by particle interactions. Often particle interactions are so dominant that they blur the quantum effects. Interactions cause quantum depletion of the condensate phase even at zero temperature. In the case of superfluid ${ }^{4} \mathrm{He}$ only a small fraction, typically around $10 \%$, is in the ground state. However, far from being only a nuisance, atomic interactions enrich the multitude of physical phenomena. They give rise to nonlinear behavior of the de Broglie matter-wave exploited in nonlinear atom optics (Section 5.4), and the strength of the interactions can even be tuned close to so-called Feshbach collision resonances (Section 6.1).

The grand canonical many-body Hamiltonian of a trapped Bose-gas, interacting through the local $s$-wave collision potential, in second quantization reads

$$
\hat{H}=\int d^{3} \mathbf{r} \hat{\psi}^{\dagger}(\mathbf{r})\left[-\frac{\hbar^{2}}{2 m} \triangle+U_{\text {trap }}(\mathbf{r})-\mu+\frac{g}{2}|\hat{\psi}(\mathbf{r})|^{2}\right] \hat{\psi}(\mathbf{r})
$$

where $\hat{\psi}(\mathbf{r})$ denotes the bosonic field atomic annihilation operator and satisfies the Heisenberg equation of motion. The interaction strength $g=4 \pi \hbar^{2} a / m$ only depends on a single atomic parameter, the scattering length a. A common approximation is the Bogolubov prescription, where the field operators describing the condensate and thermal phase can be decomposed into a complex function $\psi_{0}(\mathbf{r}) \equiv<\hat{\psi}(\mathbf{r})>$ called condensate wavefunction which can be chosen as the order parameter of the system and into a small perturbation $\delta \hat{\psi}(\mathbf{r}) \equiv \hat{\psi}(\mathbf{r})-\psi_{0}(\mathbf{r})$ describing the thermal excitations. At zero temperature, we can neglect the thermal excitations 44 and our system is completely described by a single wavefunction $\psi_{0}(\mathbf{r}, t)$ that follows the Gross-Pitaevskii equation,

$$
\left[\frac{-\hbar^{2}}{2 m} \Delta+U_{\text {trap }}(\mathbf{r})+g\left|\psi_{0}(\mathbf{r}, t)\right|^{2}\right] \psi_{0}(\mathbf{r}, t)=i \hbar \frac{\partial}{\partial t} \psi_{0}(\mathbf{r}, t) .
$$




\subsubsection{Semiclassical Approximation}

If we additionally assume $\hbar \omega \ll k_{B} T$, we can apply the semiclassical WKB approximation, i.e. the atomic motion does not have to be quantized and the trap has a continuous energy spectrum. We can then replace coordinate and momentum operators by their expectation values and, with the abbreviations $\mathcal{L}(\mathbf{r}, \mathbf{p}) \equiv \mathbf{p}^{2} / 2 m+$ $U_{\text {trap }}(\mathbf{r})-\mu+2 g n(\mathbf{r})$ and $n(\mathbf{r})=n_{0}(\mathbf{r})+n_{\text {th }}(\mathbf{r})$, we get a set of two semiclassical Bogolubov equations

$$
\begin{aligned}
& \mathcal{L}(\mathbf{r}, \mathbf{p}) u(\mathbf{r}, \mathbf{p})-g n_{0}(\mathbf{r}, \mathbf{p}) v(\mathbf{r}, \mathbf{p})=\varepsilon(\mathbf{r}, \mathbf{p}) u(\mathbf{r}, \mathbf{p}), \\
& \mathcal{L}(\mathbf{r}, \mathbf{p}) v(\mathbf{r}, \mathbf{p})-g n_{0}(\mathbf{r}, \mathbf{p}) u(\mathbf{r}, \mathbf{p})=-\varepsilon(\mathbf{r}, \mathbf{p}) v(\mathbf{r}, \mathbf{p}),
\end{aligned}
$$

where the phonon creation amplitude $u(\mathbf{r}, \mathbf{p})$ and the phonon annihilation amplitude $v(\mathbf{r}, \mathbf{p})$ obey the normalization condition $u(\mathbf{r}, \mathbf{p})^{2}-v(\mathbf{r}, \mathbf{p})^{2}=1$. They relate the particle distribution function $F(\mathbf{r}, \mathbf{p})$ and the quasiparticle distribution function $f(\varepsilon)=\left(e^{\beta \varepsilon(\mathbf{r}, \mathbf{p})}-1\right)^{-1}$ by

$$
F(\mathbf{r}, \mathbf{p})=\left(|u(\mathbf{r}, \mathbf{p})|^{2}+|v(\mathbf{r}, \mathbf{p})|^{2}\right) f(\varepsilon) .
$$

The spatial distribution of the thermal density is calculated from

$$
n_{t h}(\mathbf{r})=h^{-3} \int F(\mathbf{r}, \mathbf{p}) d^{3} \mathbf{p}
$$

and analogously for the momentum distribution. The last equation represents a generalization of equation (2.30) for interacting particles at all excitation energies. The Bogolubov equations (2.46) yield a simple expression for the excitation spectrum

$$
\varepsilon(\mathbf{r}, \mathbf{p})=\sqrt{\mathcal{L}(\mathbf{r}, \mathbf{p})^{2}-g^{2} n_{0}^{2}(\mathbf{r})} .
$$

They can be solved numerically [45] or be approximated analytically. All thermodynamic quantities can be derived from the distribution functions and the spectrum. For example the entropy reads $S=k_{B} h^{-3} \int\left(\beta \varepsilon f(\varepsilon)-\ln \left(1-e^{-\beta \varepsilon}\right)\right) c$ the heat capacity is $C=T(\partial S / \partial T)_{N}$, and the total energy follows from $C=(\partial E / \partial T)_{N}$. For homogeneous systems, where the wavefunctions are plane waves, the energy spectrum takes the well-known local-density form of the Bogolubov dispersion relation,

$$
\varepsilon(\mathbf{r}, \mathbf{p})=\sqrt{\frac{\mathbf{p}^{2}}{2 m}\left(\frac{\mathbf{p}^{2}}{2 m}+2 g n_{0}(\mathbf{r})\right)},
$$

which has been used to calculate particle-like excitations, $\mathbf{p}^{2} / 2 m \gg g n(\mathbf{r})$, and phonon-like excitations, $\mathbf{p}^{2} / 2 m \ll$ $g n(\mathbf{r})$ (Sections 4.3.1 and 5.4.2).

Several interesting results can be obtained by restricting the analysis to energies that are much larger than the chemical potential, $\varepsilon(\mathbf{r}, \mathbf{p}) \gg \mu$. From the Bogolubov equations, we then derive a particularly simple Hartree-Fock type spectrum

$$
\varepsilon(\mathbf{r}, \mathbf{p})=\frac{\mathbf{p}^{2}}{2 m}+U(\mathbf{r})+2 g n(\mathbf{r}) .
$$

As a rough approximation, above $T_{c}$, we can neglect the influence of the interactions on the density distribution, plug the semiclassical expression (2.30) into the Hamiltonian and recalculate the thermodynamic potentials with the effective potential $U_{\text {eff }}(\mathbf{r})=U(\mathbf{r})+g \lambda_{d B}^{-3} g_{3 / 2}\left[e^{\beta(\mu-U(\mathbf{r}))}\right]$ [35]. In the case of a harmonic potential, the condensate fraction is

$$
\frac{N_{0}}{N}=1-\left(\frac{T}{T_{c}^{0}}\right)^{3}-4 \frac{a}{\lambda_{d B}}\left(\frac{T}{T_{c}^{0}}\right)^{7 / 2},
$$

where the $T_{c}^{0}$ is the critical temperature in the ideal gas limit (Table 1). The critical temperature modified by interactions is estimated from (2.52) by setting $N_{0}=0$. For positive scattering lengths, the phase transition occurs 
at lower temperatures. This can be understood intuitively, because the repulsive particle interaction associated with positive scattering lengths counteracts the density compression required for initiating the condensation process.

Giorgini et al. 45 numerically integrated the semiclassical Bogolubov equations and derived the density distributions and the main thermodynamic quantities for atoms trapped in harmonic potentials. Among other results, they found that repulsive interactions strongly enhance the thermal depletion of the condensate. They also confirmed the decrease of the transition temperature and noticed a smoothing of the temperature dependance of the heat capacity at the phase transition due to collisions. For ideal gases, we set $g \rightarrow 0$ in the Bogolubov equations (2.46) and recover the results of the previous sections. The excitation spectrum is simply,

$$
\varepsilon(\mathbf{r}, \mathbf{p})=\frac{\mathbf{p}^{2}}{2 m}+U(\mathbf{r})-\mu .
$$

\subsubsection{Attractive Interactions}

The atomic interaction potential decides on the value of the scattering length $a$ : A repulsive potential corresponds to a positive $a$. For a purely attractive potential that supports no bound state $a$ is negative, and for an attractive potential that supports bound states $a$ can be either positive or negative depending on the proximity of the last bound state to the dissociation limit.

A negative scattering length may, at first, seem desirable, because it rises the BEC threshold temperature according to equation (2.52). However, attractive interactions raise other problems. The interaction energy of a Bose-Einstein condensate is given by $4 \pi \hbar^{2} a n / m$ and, if the scattering length is negative, decreases with increasing density $n$. The condensate attempts to lower its interaction energy by increasing its density until it collapses [46] by inelastic two-body spin exchange processes or three-body recombination processes. This holds for homogeneous condensates. However, when confined in a trap, the zero-point energy exerts a kinetic pressure which balances the destabilizing influence of the interactions, so that small condensates are expected to be stable. Calculations for spherical traps predict [47] $N_{\text {min }} \approx 0.575 a_{\text {trap }} /|a|$, where $a_{\text {trap }}=\sqrt{\hbar / m \omega_{\text {trap }}}$.

\subsection{Classification of Phase Transitions}

Ehrenfest Classification. - Because of the huge variety of phase transition phenomena, a general classification is not easy. The first attempt was undertaken in 1933 by Ehrenfest. He proposed the following classification scheme founded on the thermodynamic properties of the phases. A phase transition is of $n^{t h}$ order if the $n^{t h}$ derivative with temperature of at least one of the state variables, e.g. chemical potential $\mu(T)$, internal energy $E(T)$ or entropy $S(T)$, is discontinuous at the transition point whereas all lower derivatives are continuous 48 . As an example: the liquid-gas phase transition is of first order, because $\partial \mu / \partial T$ is discontinuous.

In order to characterize the Bose-Einstein phase transition, we investigated the temperature dependence of the heat capacity in Section 2.2 (Table 1 and Figure 11). We saw that, depending on the type of the confining potential, the occurrence of a thermodynamic phase transition can be quite remarkable through a discontinuity of the heat capacity $C(T)=\partial E / \partial T$ at the critical temperature $T_{c}^{0}$. For a generic power law potential, the discontinuity depends on the confinement power $\eta$. If the confinement power is $\eta \leq 2$, for example for a homogeneous system (3D-box), the discontinuity of $C(T)$ disappears, but $\partial C / \partial T$ remains discontinuous. However, in any case the chemical potential exhibits an abrupt change of its temperature dependence at $T_{c}^{0}$, i.e. $\partial \mu / \partial T$ is discontinuous. This aspect is very similar to liquid-gas phase transitions. Therefore, adopting Ehrenfest's classification scheme, Bose-Einstein condensation of an ideal gas takes place as a first order phase transition regardless of the shape of the confining potential.

The Bose-Einstein condensation of a homogeneous system is often called a condensation in momentum space, because the phases do not separate 49 . It is important to note, that the phase separation is not an essential feature for BEC and does even occur in a homogeneous system under the influence of gravity [32], because the dense condensate has a negative buoyancy inside the normal fraction. In harmonically trapped gases, the condensed and thermal fractions spatially separate to a large extent, since the condensate nucleates at the 
center of the thermal cloud, where the density is highest. The process must then be considered a condensation in phase space.

We have seen in the Sections 2.4 and 2.6, that atomic interactions and finite ensemble sizes smooth out the discontinuities. As a result, in Ehrenfest's classification scheme first order transitions become second or higher order transitions [32]. The different classification suggests a fundamental change in the quality of the transition due to interactions. We should, however, keep in mind that the reason for the occurrence of the BEC phase transition is the symmetry of the bosonic single-particle wavefunction, and that forces between the particles rather tend to blur the quantum statistical nature of the process. In the case of the strongly interacting liquid ${ }^{4} \mathrm{He}$, the heat capacity changes smoothly and exhibits a $\lambda$-shaped peak at the critical point.

Ginzburg-Landau Classification. - Landau emphasized the role of symmetry in thermodynamics by introducing the notion of the order parameter [50], which he defined as a very general macroscopic measure for the amount of symmetry in a system. Symmetry considerations play an important role at phase transitions, and many types of phase transitions change the symmetry of the system. Typically the phase with the higher temperature is more symmetric. The order parameter is zero for this phase and non-zero for the less symmetric phase. An order parameter can also be defined, if the symmetry apparently does not change as it is the case for the phase transition from liquid to gas: Both phases are isotropic. Order parameters may be very different in nature, depending on the specific system and type of force driving the phase transition, e.g. they may be $c$-numbers, vectors or even many-body quantum fields. In the case of Bose-condensation, the condensate density is often taken as the order parameter. When crossing the phase transition from high to low temperatures, the system can spontaneously adopt a symmetry that its Hamiltonian does not have, i.e. the symmetry is broken, and the order parameter takes a value different from zero. For example: the transition from liquid to solid breaks the translational symmetry.

Landau labeled a transition first order, if there is a discontinuous change in the order parameter and continuous transition or critical phenomenon, if the order parameter goes smoothly to zero at $T_{c}$. Applying the Landau criterion to Bose-gases, we find that BEC is a second-order phase transition, because the temperature dependence of the order parameter is continuous at the critical point regardless of whether the gas interacts or not (Figure (1). The Landau classification seems therefore more appropriate for the Bose-Einstein phase transition. 


\section{Chapter 3}

\section{Making and Probing Bose-Einstein Condensates}

The first hint, that Bose-Einstein condensation was more than just a theoretical fantasy came from London 49] who connected the newly found phenomenon of superfluidity in ${ }^{4} \mathrm{He}$ to BEC. However, the interpretation of the $\lambda$ point in terms of BEC was not obvious because strong particle interaction blur the quantumstatistics, and the thermodynamic quantities exhibit divergences at $T_{c}$ rather than discontinuities as expected from ideal gas BECs. The occurrence of BEC is inferred from its influence on the bulk properties of the system. These uncertainties motivated intense search in other systems. In 1954, Schafroth pointed out that electron pairs can be viewed as composite Bosons and might Bose-condense at low temperatures [51]. In 1957, Bardeen, Cooper and Schrieffer developed the microscopic theory of superconductivity [52], a phenomenon that has been related to Bose-condensation of electron- or Cooper-pairs by other researchers including Blutt, Schaffrot, Fröhlich and Bogolubov.

Motivated by the need to test the concept of composite-particle or quasi-particle condensation in weakly interacting systems, in 1962, Blatt et al. proposed the investigation of BEC in exciton gases [53]. Excitons are bound electron-hole pairs that can form a weakly interacting gas in certain nonmetallic crystals. They are interesting because their small mass permits BEC at high temperatures, their density can be controlled over a wide range by modifying the optical excitation level, and they are destructible. Excitons were discovered in 1968 and the first evidence of Bose-Einstein condensation of biexciton-molecules in $\mathrm{CuCl}$ crystal dates from 1979 [54. One year later, the influence of Bose-Einstein statistics on orthoexcitons $(S=1)$ was observed by Hulin et al. in $\mathrm{CuO}_{2}$, and finally the condensation of paraexcitons $(S=0)$ in $2 \mu \mathrm{m}$ thick stressed $\mathrm{CuO}_{2}$ films by Lin et al. [55]. They achieved BEC at densities above $10^{19} \mathrm{~cm}^{-3}$ and transition temperatures close to $T_{c}=50 \mathrm{~K}$.

Hecht [56] suggested in 1959, followed by Stwalley and Nosanow [57] in 1976, that spin polarized atomic hydrogen would be a suitable candidate for BEC. In 1978 Greytak and Kleppner at the MIT started intensive efforts to form BECs in dilute hydrogen gases. In the nineties, advances in cooling atoms by laser light led to really low temperatures, and the invention of traps for neutral atoms allowed their confinement and the compression of their density. This initiated efforts to try to realize BEC in alkalis, which have an electronic level scheme that lends itself to optical cooling. Later, it turned out that the phase space density in optical traps is limited by optical rescattering effects. As a solution to this problem people started to trap the atoms by their magnetic dipole moment and to replace optical cooling by evaporative cooling. This was the final step towards BEC in alkali gases. The hydrogen experiment, that initially stimulated the alkali experiments, now taking advantage from their success has led to BEC, as well.

Today, hybrid optical plus evaporative cooling in alkali-metals has increased the phase space density by a factor of $10^{15}$. BEC has been achieved in rubidium, lithium, sodium and also in hydrogen. In the present chapter, we will outline the experimental progress that led to BEC in the alkalis by discussing the various techniques employed. 


\subsection{Techniques for Cooling and Trapping}

Light interacts in two different ways with the mechanical degrees of freedom of atoms [58]. One way is through the force

$$
\mathbf{F}=-\nabla_{r}(\mathbf{d} \cdot \mathbf{E})
$$

where $\mathbf{d}$ denotes the atomic dipole moment and $\mathbf{E}$ the electric field of the light. The dipole force arises from the interaction of the light with the dipole, which the light induces in the atom [59]. It can be understood as stimulated scattering of photons between the modes of the light field by the atoms. The force acts in the direction of the intensity gradient. It is a conservative spatially varying force and therefore interesting for realizing trapping potentials for atoms 60. A light field with intensity $I$, detuned from a resonance $\omega_{0}$ by $\Delta=\omega-\omega_{0}$, gives rise to the dipole force

$$
\mathbf{F}=d\left(\nabla_{r} E_{0}\right) \frac{2 \Omega \Delta}{\Gamma^{2}} \frac{\sigma(\Delta)}{\sigma_{0}}
$$

where the absorption profile is described by the optical cross-section $\sigma$,

$$
\sigma(\Delta)=\frac{\sigma_{0} \Gamma^{2}}{4 \Delta^{2}+2 \Omega^{2}+\Gamma^{2}}
$$

and $\sigma_{0}=3 \lambda^{2} / 2 \pi$ is the resonant optical cross-section on a transition whose Clebsch-Gordon factor is equal to one. Furthermore, the Rabi frequency is introduced by $\Omega \equiv \mathbf{d} \cdot \mathbf{E} / \hbar=\sqrt{\sigma_{0} \Gamma I / \hbar \omega}$. The force (3.2) can be expressed as the gradient of the conservative trapping potential

$$
U=\frac{\hbar \Delta}{2} \ln \left(1+\frac{2 \Omega^{2}}{4 \Delta^{2}+\Gamma^{2}}\right)
$$

The second force is called radiation pressure and comes from spontaneous scattering of photons at an atomic resonance. It was first observed as early as 1933 by Frisch 61. The absorption of a photon from the light field (wavevector $\mathbf{k}$ ) imparts a recoil momentum $\mathbf{p}=\hbar \mathbf{k}$ to the atom. The subsequent spontaneous emission is isotropic in the time-average, so that in the average over many emission processes no momentum is transferred to the atom. The radiation pressure is dissipative and has been used in optical cooling schemes 62, 63.

The radiation pressure is velocity dependent. The velocity dependence comes from the Doppler effect, which links the external degrees of freedom of the atom (its motion) to the internal ones (detuning between light and atomic resonance frequency): The frequency $\omega$ of a light field is increased or decreased in the inertial system of the atom, i.e. relative to the atomic resonance frequency, depending on whether the atom moves towards or away from the light propagation direction. In a red-detuned light field photons are only absorbed by counterpropagating atoms, while copropagating atoms are out of resonance. One can therefore use the radiation pressure to manipulate the velocity of the atoms and if need be decelerate them. Often the reduction in kinetic energy is accompanied by a reduction in kinetic energy spread. Those cases are called optical cooling. Radiation pressure has been used to decelerate atomic beams in Zeeman-slowers 64 and chirped-frequency slowers 65.

The radiation pressure force of a light field on a two-level atom (velocity $\mathbf{v}$, linewidth $\Gamma$ ) averaged over many absorption-spontaneous emission cycles, is 66.

$$
\mathbf{F}=\hbar \mathbf{k} \frac{I}{\hbar \omega} \sigma(\Delta-\mathbf{k} \cdot \mathbf{v})
$$

The cooling force is proportional to the laser intensity, as long as the transition is not saturated, $I / I_{s}=2 \Omega^{2} / \Gamma^{2}<$ 1. The smallest temperature that two-level atoms can attain by Doppler cooling is limited by diffusion of the momentum in phase space due to the stochastic process of spontaneous emission. Cooling and diffusion heating are leveled when the atom has the kinetic energy $\mathbf{p}^{2} / 2 m=\hbar \Gamma / 2$. 


\subsubsection{Magneto-Optical Traps}

A frequently used optical cooling scheme for low temperatures consists of irradiating the atoms with three orthogonal pairs of counterpropagating red-detuned laser beams. Radiation pressure slows down the atoms without confining them, and the atoms move like in a viscous medium, the so called optical molasses [67. Surprisingly, the temperatures measured in optical molasses were well below the Doppler limit. The responsible cooling mechanisms have been identified to be based on optical pumping between the Zeeman sublevels induced by polarization gradients 68, 69. These polarization gradients are also responsible for the low temperatures found in Magneto-Optical Traps (MOT). The MOT was invented by Dalibard and first realized by Raab et al. [70] and is presently the most commonly used trap for atoms. It consists of a magnetic field gradient produced by a quadrupole field and three pairs of circularly polarized, counterpropagating optical beams, detuned red from an atomic transition and intercepting at right angles in the position of the magnetic field zero. The MOT exploits the position-dependent Zeeman shifts of the electronic levels when the atom moves in the radially increasing magnetic field. The use of circularly polarized, slightly red-detuned light, $\Delta \approx \Gamma$, results in a spatially dependent transition probability whose net effect aims at producing a restoring force that pushes the atoms towards the origin. The force exerted by one of the laser beams (wavevector $\mathbf{k}=k \hat{\mathbf{e}}_{z}$ ) acts primarily on atoms with velocity $\mathbf{v}=v \hat{\mathbf{e}}_{z}$,

$$
F_{z}=\hbar k \frac{I}{\hbar \omega} \sigma\left(\Delta-k v_{z}-\hbar^{-1} \mu z \partial_{z} B\right)
$$

Analogous expressions hold for all other beams. For small displacements and velocities the total force can be linearized,

$$
m \ddot{z}=F_{z}+F_{-z}=-\alpha \dot{z}-\varkappa z,
$$

where $\alpha$ and $\varkappa$ denote the friction and the spring constant respectively, and describe a damped motion inside a harmonic potential,

$$
\varkappa=\frac{16 \hbar k \Gamma \Omega^{2} \Delta\left(\partial_{z} \omega_{Z e e m a n}\right)}{\left(4 \Delta^{2}+12 \Omega^{2}+\Gamma^{2}\right)^{2}} \quad, \quad \alpha=\varkappa \frac{k}{\partial_{z} \omega_{\text {Zeeman }}} .
$$

The dissipative character of the MOT makes it a very powerful and versatile tool: At the same time, the MOT traps up to $10^{9}$ atoms from the environment, cools them down to very low temperatures and then confines them in a potential at densities approaching $10^{10} \mathrm{~cm}^{-3}$. However, at such high density the atomic cloud gets so optically thick, that photons are scattered several times before they find their way out. This phenomenon is termed radiation trapping. The atomic repulsion induced by the photons at each absorption and emission blows up the cloud size 771. To overcome this radiation trapping, Ketterle et al. [72 proposed to keep the colder atoms that are close to the origin of the trap in a dark electronic state in order to prevent them from scattering light. His scheme, presently known as dark MOT or dark Spontaneous Force Optical Trap (dark SPOT), takes advantage of the large hyperfine splitting of the ${ }^{2} S_{1 / 2}$ ground state, which allows to selectively excite and pump both hyperfine substates.

Typically, dark MOTs capture up to $5 \cdot 10^{9}$ atoms at densities approaching $n=10^{11} \mathrm{~cm}^{-3}$ and temperatures below $100 \mu \mathrm{K}$. The phase space density for such clouds is $\rho=n \lambda_{d B}^{3}<10^{-6}$, which is still more than six orders of magnitudes away from BEC. Since it seems impossible to reach BEC in MOTs, alternative trapping schemes have been developed, the most promising of which are dipole force traps using laser light and magnetic traps operating without light beams at all.

\subsubsection{Far-off Resonance Dipole Traps}

For large detunings, the potential depth (or light-shift) estimated from equation (3.4) reads:

$$
U=\frac{\hbar \Omega^{2}}{4 \Delta}
$$

while the spontaneous light scattering rate $\gamma_{s}$ is proportional to $I / \Delta^{2}$. Heating of the atoms due to spontaneous scattering of photons can therefore be avoided by using intense, far-detuned laser beams. Such dipole force trapping potentials are called Far-off Resonance Traps (FORT) and can be engineered with various geometries. 
For example, one-, two- or three-dimensional configurations of red-detuned standing light waves give rise to arrays of potential valleys in the intensity antinodes called optical lattices [73, 74]. The simplest optical dipole trap (and the first that has been realized [60]) consists of a tightly focussed red-detuned laser beam, that confines the atoms at its waist. Atoms trapped in a crossed dipole beam trap have even been evaporatively cooled 75 . Alternatively, one can use blue-detuned FORTs, where the atoms are confined in local minima of the intensity profile and suffer less from spontaneous light scattering [76]. Finally, dipole beams can be used in conjunction with other trapping techniques (a blue-detuned FORT was used to repel atoms from the center of a magnetic quadrupole trap [4]) (Section 3.2.1), to manipulate Bose-Einstein condensates [77] (Section 4.3.1) and even to trap them [78] (Section 4.2.2).

The optical approach offers the advantage of high spatial definition and temporal control, e.g. the trap depth and location can easily be manipulated and modulated. The trap can be turned on and off very fast compared to magnetic traps and offers the advantage of being insensitive to magnetic fields, i.e. all magnetic substates can be trapped. Furthermore, optical subrecoil cooling schemes that do not work for magnetically trapped atoms, may be implemented in optical traps. Velocity Selective Coherent Population Trapping (VSCPT) led to extremely low temperatures in the nano-Kelvin regime [79] and Raman cooling led to very high phase-space densities [80]. Several groups attempt to attain BEC using all-optical methods, and it seems possible to cross the phase transition in the near future [81].

\subsubsection{Magnetic Traps}

Magnetic traps hold the atoms by their magnetic dipole moment $\boldsymbol{\mu}=\mu_{B} g_{F} \mathbf{F}$, where $\mu_{B}$ denotes the Bohr magneton and $g_{F}$ is the Landé $g$-factor for the total atomic spin $\mathbf{F}=\mathbf{J}+\mathbf{I}=(\mathbf{L}+\mathbf{S})+\mathbf{I}$. The symbol $\mathbf{I}$ denotes the nuclear spin, $\mathbf{S}$ is the electron spin, and $\mathbf{L}$ is the electron angular momentum. The Landé factor can be calculated from

$$
\begin{aligned}
g_{F} & \approx g_{J} \frac{F(F+1)+J(J+1)-I(I+1)}{2 F(F+1)}, \\
g_{J} & =1+\frac{J(J+1)+S(S+1)-L(L+1)}{2 J(J+1)} .
\end{aligned}
$$

If the spin adiabatically follows the magnetic field, the force that the magnetic field gradient exerts on an atom is

$$
\begin{aligned}
\mathbf{F}_{\text {magn }} & =-\nabla \mathbf{r} U, \\
U & =-\boldsymbol{\mu} \cdot \mathbf{B}=-g_{F} m_{F} \mu_{B} B .
\end{aligned}
$$

Thus, depending on the atom's magnetic sublevel $m_{F}$ (positive or negative), it is attracted towards or repulsed from a magnetic field extremum. Unfortunately, static magnetic field maxima are not possible, so that only lowfield seekers can be trapped in magnetic field minima. These have the disadvantage of not being the energetically lowest state. The spontaneous decay rate out of those metastable Zeeman states is only $\sim 10^{-10} \mathrm{~s}^{-1}$, but spinchanging collisions can induce decay and trap losses. Even in the lowest atomic state the trapped alkali gases are metastable: In three-body recombination processes two atoms can form an energetically more favorable dimer if a third atom is nearby to take away the excess energy. Since the event rate for three-body collisions scales with the third power of the density, this process becomes dominant at high densities [82], e.g. for Bose-Einstein condensates.

Dynamic traps based on time-dependent magnetic fields have been proposed for both low- and strong-field seekers by Lovelace et al. 83. The first demonstration of a static magnetic trap for neutral atoms 84 used an anti-Helmholtz coil configuration to produce an axially symmetric quadrupole field. The magnetic field geometry of a spherical quadrupole trap has a linear spatial dependence and provides the tightest confinement for atoms.

The assumption that the spin adiabatically follows the magnetic field, unfortunately does not hold in all cases. Especially, near zero magnetic field the Larmor frequency $g_{F} m_{F} \mu_{B} B / \hbar$ may get smaller than the rate of change of magnetic field amplitude experienced by the atom through its motion with velocity $v$, i.e. $v \partial_{r} B / B$. 
At this location, missing a quantization axis, the atoms are free to reorient themselves arbitrarily and undergo so called Majorana spin flip transitions to untrapped magnetic sublevels. This is exactly the case for a quadrupole trap. In this trap, atoms are lost due to Majorana spin flips as they pass near the trap center due to the sudden change of the magnetic field. The colder the atoms are, the more time they spend near the center "hole", and the situation is even worse.

The Time-Orbiting-Potential (TOP) trap was designed to suppress the Majorana loss by adding a rotating transverse bias field $B_{\text {bias }}$ to the quadrupole trap (gradient $\partial_{r} B_{\text {quad }}$ ) [85]. The bias field shifts the "hole" away from the region where atoms are trapped to a distance $r_{d}=B_{\text {bias }} / \partial_{r} B_{\text {quad }}$ from the center. The hole rotates in a so called death circle around the harmonic trap, fast enough for the atoms to only respond to the time-averaged potential. An alternative approach are Ioffe-Pritchard (IP) type magnetic field configurations. In the magnetic field minimum the field amplitude does not vanish, so that Majorana spin-flip transitions do not occur. The original IP trap consists of a combination between a quadrupole waveguide and a magnetic bottle [86, 87]. Variations of IP traps include the cloverleaf trap [88, the baseball trap [7, 89], the Ioffe bar trap [90, and the Quadrupole Ioffe Configuration (QUIC) trap [91].

Laser cooling in magnetic traps has the advantage over evaporative cooling (next section), that the heat dissipation does not rely on the irreversible removal of hot atoms and does not require high densities and collision rates. On the other hand, Doppler-cooling in magnetic traps is possible [92], but it meets its natural limit at temperatures in the milli-Kelvin range. Raman-cooling and VSCPT are not compatible with magnetic traps. However, other cooling schemes are possible or will probably be developed in the future. One example is the idea of gravitational Sisyphus cooling tested by Newbury et al. [93].

\subsubsection{Evaporative Cooling}

As we have seen earlier, laser cooling becomes ineffective when the density of the gas is high. We need another dissipative mechanism to cool atoms confined in magnetic traps. A new method called evaporative cooling has been proposed by Hess [94 for spin-polarized hydrogen $(\mathrm{H} \uparrow)$ and has been observed by Masuhara et al. [95]. It was later utilized for the alkali-metals [75, 85, 96]. A detailed review has been published by Ketterle and van Druten [97].

Evaporation always occurs when energetic particles leave a system with finite binding energy, taking away more than their share in mean energy per particle. We consider here the case of a magnetic trapping potential with a finite extension, i.e. the potential has an edge or a spout through which hot atoms with enough kinetic energy to reach that region can leave the trap. In the ideal case, this will lead to a complete truncation of the hot tail of the equilibrium Maxwell-Boltzmann velocity distribution. If the remaining system finds back to thermal equilibrium, it will do so at a lower temperature. The redistribution of kinetic energy among the atoms that ultimately leads to rethermalization, happens through elastic collisions. It takes about three collisions per atom to rethermalize a cloud 89, 98. The rate for elastic collisions between trapped atoms is

$$
\gamma_{\text {coll }}=n_{0} \sigma_{e l} \bar{v} \sqrt{2} \sim \rho^{3} N^{2 / 3},
$$

where $n_{0}$ is the peak density, $\sigma_{e l}$ is the elastic scattering collision cross-section, and $\bar{v}$ is the average relative velocity between two atoms [97]. Obviously, the evaporation process slows down when the cloud gets colder, unless the potential edge is lowered so that the hottest atoms of the colder cloud are evaporated. By continuously lowering the potential edge while the atomic cloud is rethermalizing (this procedure is called forced evaporation), very low temperatures in the nano-Kelvin regime can be reached and the phase space density can be increased by six orders of magnitude up to the threshold of Bose-Einstein condensation. This is of course only possible by sacrificing many hot atoms. Even in a well optimized evaporation ramp, only $1 \%$ may reach the condensate stage after about 500 collisions per atom.

Two aspects should be pointed out concerning the optimization of the evaporation path, i.e. the downramping of the truncation edge. The first aspect is, that inelastic collisions with atoms from the background vapour limit the trap lifetime. Therefore, the evaporation needs to be fast, which requires high elastic collision rates or a good vacuum. There is a trade-off between an efficiently slow evaporative cooling on one hand and avoiding the accumulation of trap losses on the other hand. The second aspect is, that the dimensionality of the evaporation edge determines the efficiency of evaporative cooling. The first demonstration of evaporation 
in $\mathrm{H} \uparrow$ ejected hot atoms over a saddle point. The saddle point constitutes a small region of space away from the trap origin, and only atoms that have enough kinetic energy in one direction $E_{z}>U_{\text {edge }}$ can leave the trap. The evaporation is then called 1-dimensional (1D). It is true, that ergodic mixing due to anharmonicities in the trapping potential will sooner or later drive all the atoms through this region, but this effect becomes less pronounced when the cloud cools down, since the atoms settle down in the harmonic (and therefore separable) region at the bottom of the trapping potential. This fact has inhibited efficient evaporation in $\mathrm{H} \uparrow$ below $120 \mu \mathrm{K}$ [99.

A second evaporation technique has been demonstrated in TOP traps [85]. As explained in Section 3.1.3, it is a feature of TOP traps to be surrounded by a death-circle that ejects the atoms passing through. This death-circle can act as a $2 \mathrm{D}$ evaporation surface if its radius $r_{d}$ is large enough. Under the influence of gravity the dimensionality is even reduced to 1D [97]. For small atomic clouds, typically less than $10^{8}$ atoms, a TOP trap only needs a moderate death circle radius. But a small death circle is an obstacle for the creation of Bose condensates with large numbers of atoms. For efficient evaporation, it is necessary for the death circle radius $r_{d}$ to be greater than 3 to 4 times the $r m s$ radius $r_{0}$ of the trapped atom cloud. On the other hand, it is also necessary that the elastic collision rate be very large. This is usually achieved in part with an adiabatic compression of the magnetic trapping potential, in which the trap frequency increases according to

$\omega_{r} \sim \partial_{r} B_{\text {quad }} / B_{\text {bias }}^{1 / 2}$. Thus, we wish to increase $\partial_{r} B_{\text {quad }}$ and decrease $B_{\text {bias }}$. However, this also shrinks the ratio $r_{d} / r_{0} \sim B_{\text {bias }}^{3 / 4} / B_{\text {quad }}{ }^{1 / 2}$. For large atom numbers the initial radius $r_{0}$ tends to be large and the ratio $r_{d} / r_{0}$ small unless the magnetic field strengths are very large. Thus, only modest compression can be achieved before the death circle loss sets in, and the elastic collision rate must already be large enough for efficient evaporation at this point. This means that for large clouds it is not possible to depend on a large compression of the density, and that the initial densities in the trap must not be too far from those required for efficient evaporation. This is achieved by efficient optical cooling and compression, and efficient transfer of the atoms into the TOP [6].

The most successful evaporation technique implemented so far is based on radiative coupling of trapped and untrapped states in an energy-selective way and is termed radiofrequency $(r f)$ evaporation. It originates from an idea proposed by Pritchard and coworkers [100], who already had some experience with rf-spectroscopy on magnetically trapped neutral atoms [101]. The spatially dependent Zeeman-splitting is an intrinsic feature of magnetic traps. Irradiation of a radiofrequency wave with a given frequency couples the trapped and untrapped Zeeman-substates at a well-defined distance from the trap origin. This gives rise to a 3D evaporation surface, where crossing atoms can undergo Landau-Zener transitions and be expelled from the trap. The technical advantages of this scheme are substantial: The magnetic trapping potential does not need to be manipulated, and the potential edge can easily be controlled by the radiofrequency. If the evaporation is forced by continuously lowering the radiofrequency and if the evaporation path is suitably chosen, the density will increase and therefore the collision rate. The rethermalization will speed up and initiate run-away evaporation. Rf-evaporation was first demonstrated by Ketterle and coworkers [96].

Another cooling mechanism based on collisions is sympathetic cooling. The technique originally used in ion traps was later applied to neutral atoms confined in magnetic traps. The idea consists of bringing the gas into thermal contact with a cold buffer gas. In some cases, the buffer gas can be optically or evaporatively cooled. Buffer gas loading of conservative traps, e.g. magnetic traps [102], is an alternative to the transfer from MOTs. Sympathetic cooling has been used in magnetic traps to create double condensates [7] (Section 4.2.1) and to cool fermions down to the regime of quantum degeneracy [103] (Section 3.2.3).

\subsection{Realization of Bose-Einstein Condensation}

In early 1995, several research groups were very close to the long pursued goal. Several improvements of the optical trap led to large atom numbers transferred to the quadrupole magnetic trap, evaporative cooling had been observed. The first observation of Bose-Einstein condensation in a dilute gas was made at JILA [3] in rubidium. It was followed by Rice [5] in lithium and MIT in sodium [4], and later by Han et al. [6] and Hau et al. [104, and has now been confirmed by more than twenty groups worldwide. This section will briefly relate the first three experiments. A remarkable achievement is the condensation of atomic hydrogen [99]. Other candidates for BEC are thoroughly investigated, like cesium [81, 105, 106], potassium [107], helium [108] and 
neon $[109$.

\subsubsection{BEC in Alkalis}

The JILA experiment led by Cornell and Wieman worked with a Rubidium vapour cell dark MOT. Operating at $10^{-11}$ torr it took $300 \mathrm{~s}$ to load $10^{7}$ atoms. In order to optimize the loading efficiency into a magnetic trap, the MOT gradient and laser frequency detuning were adjusted and a short pulse of circularly polarized laser light pumped the atoms in presence of a weak homogeneous magnetic field defining the quantization axis into the fully stretched $F=2, m_{F}=2$ Zeeman state. Then, all laser light was switched off and the quadrupole TOP trap quickly switched on. The effective time-averaged potential $\mu_{B} B$ was pancake-shaped with secular frequencies close to $\omega_{z}=\sqrt{8} \omega_{r}=2 \pi \times 120 \mathrm{~Hz}$. The TOP trap rotating frequency $\omega_{T O P}=2 \pi \times 7.5 \mathrm{kHz}$, was chosen to satisfy $\hbar \omega_{z} \ll \hbar \omega_{T O P} \ll \mu_{B} B$. The potential was adiabatically compressed by ramping up the quadrupole field gradient and then reducing the rotating bias field amplitude. This enhanced the collision rate to about three per second, which was (in view of the $70 \mathrm{~s}$ magnetic trap lifetime) enough to initiate run-away evaporative cooling. At this stage, the cloud consisted of $4 \times 10^{6}$ atoms at $90 \mu \mathrm{K}$ temperature and $2 \times 10^{10} \mathrm{~cm}^{-3}$ density. Death circle induced Majorana spin flips and $r f$-induced Landau-Zener transitions both acted as an edge to the potential and contributed to evaporative cooling. The $r$-scalpel was ramped down thus skimming off the hot atoms from the continuously rethermalizing cloud. The phase transition was crossed with the $r$ f-frequency at $3.6 \mathrm{MHz}$. With a $5 \mathrm{G}$ rotating bias field amplitude, this $r$-frequency made the trapping potential about $800 \mathrm{kHz}$ deep. Finally, after an equilibration time of two seconds, the released and ballistically expanded condensate was probed after $40 \mathrm{~ms}$ time-of-flight with a circularly polarized laser beam tuned to a cycling transition (Section 3.3.1). Figure 1 shows typical absorption pictures taken with that method. The signatures of BEC are 1) a bimodal density distribution with a sharp increase in peak density in the center of the cloud, 2) a critical dependence on the final $r$-ramp frequency, and 3) an anisotropic shape of the central condensed feature. As discussed later (Section 4.1), only the BEC wavepacket remembers the shape of the trap that confined it before being released.

Of course, the condensed atom number and quality of the results in general have been largely improved in subsequent experiments at JILA and in other groups. E.g. the loading of the optical trap from the background vapour has been replaced by a Zeeman-slower, a double MOT or an axicon trap configuration which permitted faster loading rates at a lower vapour pressure. Other beam and magnetic trap configurations have been used, and other imaging systems have been developed. Today, atom numbers higher than $10^{6}$ can routinely be condensed in TOP traps. However, the essential features of the method described above has been the same for all alkali BEC experiments.

The Rice group led by Hulet opted for lithium [5]. Lithium has a slightly negative scattering length $a=$ $-27.3 a_{B}$, for which only small condensates are expected to be stable (Section 2.6.2). The Rice group used a magnetic trap configuration made of permanent magnets in an arrangement that produces a harmonic potential with a magnetic field minimum offset by $823 \mathrm{G}$. This has the advantage of a simple experimental setup, but at the price of flexibility. The magnetic trap is directly loaded from a Zeeman-slower. A $10^{-12}$ torr background pressure corresponding to $10 \mathrm{mn}$ magnetic trap lifetime allows 1000 collisions per lifetime with an elastic scattering cross-section of $\sigma=5 \times 10^{-13} \mathrm{~cm}^{2}$. After typically $5 \mathrm{mn}$ evaporation, a sample of $10^{5}$ atoms reaches temperatures close to $300 \mathrm{nK}$. Because the magnetic field cannot be switched off, in-situ imaging of the dense condensed cloud is necessary. Near-resonant imaging of the optically thick cloud introduces lensing effects, which make the interpretation of the recorded images difficult 110 and first led to erroneous claims about the numbers of condensed atoms [5]. Later, the use of phase-contrast imaging which exploits the birefringence of the atoms in the strong magnetic field offset (Section 3.3.2) resolved this problem and resulted in the observation of limited atom numbers of about 1400 atoms. Subsequent experiments monitored the dynamics of collective collapse of lithium BECs as soon as a critical atomnumber is surpassed 111.

The MIT group led by Ketterle used sodium. Instead of using a vapour cell, they loaded their MOT from a Zeeman slower. Apart from this, their approach was similar to the JILA experiment, except for the method used for suppressing the Majorana spin flips. In their first experiment they used a far-off resonant optical beam, $\lambda=514 \mathrm{~nm}$ and $I=2 \times 10^{5} \mathrm{~W} / \mathrm{cm}^{2}$, causing $7 \mathrm{MHz}$ light shift to repel the atoms from the center of their

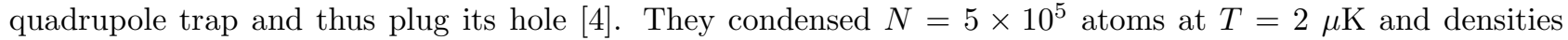
around $n=4 \times 10^{14} \mathrm{~cm}^{-3}$. In a modified setup, the MIT group replaced the quadrupole trap by a cloverleaf 
trap, which has a Ioffe-Pritchard type potential [88] and is free of the spin-flip problem (Section 3.1.3). In this trap, Mewes et al. produced condensates of $N=5 \times 10^{6}$ atoms. In contrast to the pancake-shaped fixed aspect ratio of TOP traps, cloverleaf traps are cigar-shaped and have a large tunable aspect ratio, which proved useful for a range of subsequent experiments.

\subsubsection{BEC in Hydrogen}

Hydrogen is a very interesting element to study BEC, because its small scattering length, $a_{s} \approx 1.23 a_{B}$, makes it an almost ideal gas. This has the advantage of small three-body losses allowing dense condensates. The small mass results in a high critical temperature. Its simple level structure allows precise calculations of the interaction potentials based on first principles, which may, in this way, be tested by experiments. The narrow $1.3 \mathrm{~Hz}$ wide $1 S-2 S$ (Lyman- $\alpha$ ) transition at $121.56 \mathrm{~nm}$ might be a good candidate for frequency standards in atomic fountains 112]. Narrow lasers with $0.6 \mathrm{~Hz}$ emission bandwidth, that would be able to exploit this narrow reference line have already been constructed in other wavelength regions 113 .

In 1978 Greytak and Kleppner started intensive efforts to form BECs in dilute hydrogen gases. Twenty years later they finally reached their goal [99]. The sequences of this difficult experiment recapitulate the advances in the historical development towards higher densities and lower temperatures. In this experiment, large numbers of hydrogen molecules were dissociated in a cryogenic discharge, spinpolarized and trapped in a magnetic Ioffe-Pritchard potential and confined in a cell with $120 \mathrm{mK}$ cold liquid ${ }^{4} \mathrm{He}$ coated walls. The atoms thermalized by collisions with the walls until they settled down in the $500 \mathrm{mK}$ deep trap and thermally disconnected from the wall. Because cryogenic cooling is limited to $40 \mathrm{mK}$, a new technique had to be invented, namely evaporative cooling [94] over a saddle point of the potential. The evaporation could be forced by lowering the trapping potential down to $1.1 \mathrm{mK}$. However, the evaporation surface is one-dimensional (Section 3.1.4) and becomes increasingly inefficient at temperatures below $120 \mu \mathrm{K}$, because rethermalizing collisions are rare due to the small scattering length. The problem was solved by applying the technique of radiofrequency evaporation, which had been developed for the alkali atoms and yields three-dimensional evaporation surfaces. Unfortunately, radiofrequency evaporation requires low-field seeking atoms which have the disadvantage of being in excited spin states and therefore metastable with respect to dipolar spinflips.

The phase transition was finally crossed at $50 \mu \mathrm{K}$ temperature and atomic densities of about $5 \times 10^{15} \mathrm{~cm}^{-3}$. The number of condensed atoms was $10^{9}$, corresponding to a limited condensed fraction of below $10 \%$. At higher condensed fractions and densities the losses due to dipolar heating overrule the gain from evaporative cooling of the thermal cloud [114], which is rather poor because of the small scattering length. The needle-shaped condensed cloud had $15 \mu \mathrm{m}$ radial and $5 \mathrm{~mm}$ axial extension.

The atomic sample was probed by ultra-high resolution two-photon Raman spectroscopy on the forbidden $1 S-2 S$ transition. The fluorescence photons could be observed by Stark-quenching the $2 S$ level via the rapidly decaying $2 P$ level. The spectrum shown in Figure 5 consists of a Doppler-sensitive and a Doppler-free part which result from photons being absorbed from the same laser beam or from counterpropagating beams, respectively. The Doppler-free narrow peak is red-shifted by cold collisions by an amount $\Delta \nu_{1 S-2 S}=-3.8 \mathrm{~Hz} \mathrm{~cm}^{3} \times n$, and its width of a few kilo-Hertz is mainly due to transit time broadening of the atoms flying through the small $50 \mu \mathrm{m}$ waist of the laser beam. The density dependence of the pressure shift is useful for studying the density distribution of the cloud. The Doppler-sensitive broad peak is blue-shifted by $6.7 \mathrm{MHz}$, i.e. twice the recoil-energy. It represents an in-situ measurement of the atomic momentum distribution and is in this respect complementary to imaging techniques that either measure the spatial distribution in-situ (Section 3.3.2) or the momentum distribution after a time-of-flight (Section 3.3.1).

The condensate leaves its imprints on the two-photon spectrum. The Doppler-free peak has a shoulder, that is red-shifted by $-0.9 \mathrm{MHz}$ and originates from a region in the trap where the density is significantly higher. This is a signature of BEC. The intrinsic width of the shoulder is determined by the position-momentum uncertainty, but is overruled by the broadening due to the very inhomogeneous density distribution of the BEC. The broad Doppler-sensitive peak develops a narrow structure that is qualitatively similar to the one of the Doppler-free peak. 


\subsubsection{Fermions}

Atoms are either bosons or fermions depending on whether their spin is integer or half-integer. At high phasespace densities the atoms have to sort out how they will organize their coexistence. Bosons encourage each other to occupy the same phase-space cell, in contrast to the reluctant fermions which follow the Pauli exclusion principle. The different behavior is described by different quantum statistics which settle how the phase-space (i.e. the available energy levels) has to be populated by the atoms. The Bose-Einstein distribution holds for bosons, the Fermi-Dirac distribution for fermions and both asymptotically approach the Boltzmann distribution at high temperatures. We have seen that bosons undergo a phase-transition and all condense in the ground state as the temperature is lowered. On the other hand, the fermions must organize their phase-space so that their energy levels are organized like a ladder. This has observable consequences at low temperatures. (1) The internal energy of a fermionic gas is a little higher than expected according to classical statistics, because the atoms drive each other out of the lowest energy levels. (2) In a confining trap, the cloud resists compression, its spatial density distribution is shaped by atoms pushing each other out of the trap center, where the potential energy is lower. (3) The collision rate is strongly suppressed, because the density cannot increase beyond a certain limit. This last point makes ultracold Fermi-gases interesting for frequency standard applications, because of the absence of pressure shifts.

It is, of course, very interesting to experimentally confirm the impact of quantum statistics on a cold Fermigas. There are various fermions among the alkali isotopes (see Table 2). DeMarco and Jin [103] carried out experiments on potassium. They loaded a magnetic cloverleaf trap from a MOT with $150 \mu \mathrm{K}$ hot atoms and initiated evaporative cooling. The problem with evaporative cooling of fermions is that at ultralow temperatures $s$-wave collisions between the spin-polarized identical fermions dominate, and those are forbidden by Pauli's exclusion law. Because $s$-wave collisions between distinguishable particles are possible, DeMarco and Jin could circumvent this problem by dividing the potassium cloud into two different internal energy states and simultaneously cooling them. The two energy states used were the $\left|F, m_{F}\right\rangle=|9 / 2,7 / 2\rangle$ and the $|9 / 2,9 / 2\rangle$ Zeeman sublevels of the ground state. Inside the magnetic trapping field the Zeeman states are split. In order to maintain a 50\% ratio during forced evaporation, the evaporation edge of both Zeeman states had to be controlled and ramped down separately and simultaneously by two microwave frequencies tuned between each of the Zeeman states and an untrapped level of the $F=7 / 2$ hyperfine state.

DeMarco and Jin cooled a two-component Fermi-gas of $7 \times 10^{5}$ potassium atoms down to $300 \mathrm{nK}$, which corresponded to $60 \%$ of the atoms populating energy levels below the Fermi-temperature $T_{F}=\hbar k_{B}^{-1}\left(6 \omega_{z} \omega_{r}^{2} N\right)^{1 / 3}$. Then they selectively removed the $|7 / 2\rangle$ atoms, took time-of-flight absorption pictures and analyzed the momentum distribution. The onset of the Fermi-statistical regime was observed as a barrier to evaporative cooling at temperatures lower than $0.5 T_{F}$ and also left its imprint on the momentum distribution. The shape of the distribution deviated from the Gaussian expected for classical gases, and the analysis of the second moment of the distribution (which is independent of any assumption concerning the exact statistical distribution) showed a higher total energy than classically expected.

A possible next step could be the attempt of inducing Cooper-pairing of fermions. Cooper-pairs are bosons and it might be possible to cool them down to a BEC phase transition. This effect is known from superconductivity, where Bose-condensed Cooper-paired electrons move through a metal without friction. The superfluidity of fermionic ${ }^{3} \mathrm{He}$ is also explained by Cooper-pairing. In dilute gases, however, this is most likely a very difficult task, due to the lack of efficient cooling mechanism at such low temperatures.

Table 2 lists the scattering lengths and transition frequencies for some bosonic and fermionic alkalis. The scattering lengths are either measured or calculated. Many calculations on scattering lengths and interaction potentials were carried out by Verhaar, Julienne, Greene, Stwalley and their respective coworkers.

\subsection{Imaging Techniques}

All methods of extracting information from a condensate used up to now are based on its interaction with light. However, the methods differ in the kind of information they yield. A detailed discussion can be found in reference [115]. We will restrict ourselves here to tracing the essential points. 
We can either use frequency-domain high-resolution Doppler-sensitive Raman-spectroscopy 99] or just take pictures of the spatial density distribution of the atomic sample. Pictures are taken by irradiating the light of a probe beam and either imaging the fluorescence of the atoms or the imprint of the atomic cloud on the intensity distribution of the probe beam. The fluorescence method does not reproduce the density distribution of optically dense clouds and is afflicted with a small light collection angle. The imprint method can be absorptive or dispersive. Absorptive imaging destroys the sample and only works for optically thin clouds. It is therefore commonly used in Time-of-Flight (TOF) schemes on ballistically expanded clouds. Dispersive imaging usually preserves the sample. It works for thick clouds and is commonly used in-situ on trapped samples with far-off resonant probe light. Several methods of dispersive imaging have been applied, notably dark-ground imaging, phase-contrast imaging and polarization-contrast imaging. It is worth noticing, that in order to get specific informations, one may manipulate the sample before imaging it. E.g. one can convert momentum into coordinate information by ballistic expansion or one can let a condensate interfere with a reference condensate and extract information about the matter-wave phase distribution.

\subsubsection{Time-of-Flight Imaging}

Time-of-flight (TOF) absorption images are taken after non-adiabatic removal of the trapping potential within a time much shorter than the trap oscillation period, $t_{\text {switch }} \ll \omega_{\text {trap }}^{-1}$, which is typically on the order of a milli-second. After a time long enough to allow the initial velocity distribution of the trapped BEC to convert into a spatial distribution of the expanded cloud that is large enough to neglect the initial spatial distribution, the cloud is irradiated with a weak resonant probe beam. The shadow imprinted by the cloud into the probe beam is imaged onto a CCD camera. Several reasons make TOF imaging well-suited for probing BECs. (1) The total number of atoms can be derived from the missing photons in the probe beam, (2) the velocity distribution directly reflects the temperature, (3) the expanded cloud is optically thin enough, not to saturate the probe beam shadow even if the probe frequency is on resonance, (4) the expanded cloud is large enough to be easily resolved with standard imaging systems and even to reveal structural details. E.g. the shapes and aspect ratios of the condensed and thermal parts are different and permit their visual separation. On the other hand, it is important for TOF imaging to guarantee the sudden and free expansion of the cloud, which is a technically demanding task.

The density of the thermal part $n_{t h}(\mathbf{r})$ of an ideal trapped Bose-gas has been estimated in the semiclassical limit (Section 2.4) by equation (2.30), while its momentum distribution $\tilde{n}_{t h}(\mathbf{p})$ follows equation (2.31). Switching off the trap suddenly removes all potential energy. While the gas ballistically expands, the self-interaction energy is adiabatically converted into kinetic energy [116]. If we wait sufficiently long, $t \gg \omega_{\text {trap }}^{-1}$, the expanded spatial density distribution $n_{T O F}(\mathbf{r}, t)$ just traces the original momentum distribution,

$$
n_{T O F}(\mathbf{r}, t)=m^{3} t^{-3} \tilde{n}_{t h}(m \mathbf{r} / t)=\left(\lambda_{d B} \omega_{t r a p}\right)^{-3} g_{3 / 2}\left(e^{\beta\left(\mu-m \mathbf{r}^{2} / 2 k_{B} T t^{2}\right)}\right) .
$$

In the far wings, $m \mathbf{r}^{2} / 2 t^{2} \gg \mu$, the density of the thermal cloud approaches a Gaussian distribution,

$$
n_{T O F}(\mathbf{r}, t) \approx \frac{N}{(2 \pi)^{3 / 2} r_{r m s}^{3}} e^{-\mathbf{r}^{2} / 2 r_{r m s}^{2}}
$$

where we used $k_{B} T \approx m \mathbf{r}_{r m s}^{2} / t^{2}$ and $N=\left(k_{B} T / \hbar \omega_{\text {trap }}\right)^{3} \exp \left(\mu / k_{B} T\right)$. The quantity $(2 \pi)^{3 / 2} r_{r m s}^{3}$ is sometimes called effective volume. If we assume thermal equilibrium between the thermal and condensed fraction, the temperature can easily be extracted from the far wings of the Gaussian (3.14), which are beyond the spatial extension of the BEC wavefunction. The generalization of the distribution (3.14) to nonisotropic potentials is straightforward. The ideal-gas approximation is valid, because the thermal fraction is very dilute compared to the condensed fraction.

While the thermal atomic cloud constitutes a statistical ensemble, the condensed part is described by a single complex wavefunction $\psi_{0}(\mathbf{r})$ that is a solution of the Gross-Pitaevskii equation (2.45). Let us assume a harmonic trapping potential $U_{\text {trap }}(\mathbf{r})$. In the absence of interactions, the ground state wavefunction is a Gaussian, and it stays a Gaussian during ballistic expansion although it changes its size and aspect ratio. In most experiments, however, the interactions are strong, $g\left|\psi_{0}(\mathbf{r}, t)\right|^{2} \gg \hbar \omega_{\text {trap }}$. In this so-called Thomas-Fermi 
limit, we can neglect the kinetic energy term in equation 2.45). The solution is a parabolically shaped density distribution, which preserves its shape during ballistic expansion [117] (Section 4.1). The chemical potential $\mu$ can be estimated in the Thomas-Fermi limit from the width of the expanded condensate $\mu \approx m \mathbf{r}_{r m s}^{2} / t^{2}$.

In summary, absorptive TOF imaging permits the unambiguous measurement of $N, N_{c}, T$, and $\mu$ and the derivation of $T_{c}$ from $N_{c}=g_{3}(1)\left(k_{B} T_{c} / \hbar \omega_{\text {trap }}\right)^{3}$. However, we still have to establish the relationship between the expanded cloud density (3.14) and the absorption profile that the cloud imprints into the probe beam.

Optical Density. - The influence of the atoms on the probe beam is described by the complex refraction index $n_{\text {ref }}$, which can be linked to the optical cross-section $\sigma(\Delta)$ and easily be calculated from the optical Bloch equations for a two-level atom in the rotating-wave approximation [118]:

$$
n_{\text {ref }} \approx 1+\frac{n(\mathbf{r}) \lambda \sigma(\Delta)}{4 \pi}\left(i-\frac{2 \Delta}{\Gamma}\right) .
$$

If the atomic cloud is small and dilute, the amplitude of the probe beam is locally attenuated and phase shifted:

$$
E=E_{0} \exp \left[\frac{2 \pi i}{\lambda} \int\left(n_{\text {ref }}-1\right) d z\right] \equiv E_{0} e^{-\alpha / 2} e^{i \delta} .
$$

The absorption coefficient or optical density $\alpha$ is the product of the column density $\int n(\mathbf{r}) d z$ and the optical cross-section. $z$ is chosen to be the imaging direction. Close to resonance, the interaction is well described by the Lambert-Beer law, $I=I_{0} e^{-\alpha}$, where

$$
\alpha(x, y)=\sigma_{0} \int n(\mathbf{r}) d z
$$

The CCD camera actually records the intensity distribution of the probe beam $I(x, y)$ that went through the atomic cloud. Inhomogeneities in the intensity distribution can be compensated by taking a reference picture without atoms and calculating $I(x, y) / I_{0}$.

\subsubsection{In-Situ Imaging}

TOF imaging is a one-shot measurement and destroys the sample. As mentioned earlier, absorptive in-situ imaging is accompanied by the problem of large local phase shifts of the probing beam due to the optical thickness of the cloud. Measurements have been carried out in this regime [104, but they have only yielded reliable values at the surface of the trapped cloud, where the density is small. The problem can be circumvented by dispersive imaging, which additionally possesses several other advantages.

If we increase the detuning, the dispersion coefficient $\delta$ defined in equation (3.16) decreases, but the absorption $\alpha$ decreases faster, because $\delta=\alpha \Delta / \Gamma$. Very far from resonance, the heating due to absorption and spontaneous emission is insignificant and the BEC will not be destroyed. The probe beam also phase-shifts the matter-wave and should have uniform intensity distribution in order to prevent the formation of matter-wave phase gradients. The local phase shift in the probe beam cross-section can be turned into an intensity profile using the method of dark ground imaging or phase contrast imaging [115.

Andrews et al. have used dispersive, non-destructive imaging techniques [119], that allows taking up to 20 images of the trapped BEC without significantly perturbing it. This permits to watch the condensate dynamics, e.g. the response to trap perturbations, on-line. Bradley et al. [110 took phase-contrast images which exploit the birefringence of the atoms in the strong magnetic field offset of their trap. Confined in a trap, the thermal and condensed fractions of a cloud are not well separated. Therefore, in-situ imaging is not well suited for determining the thermodynamic quantities at the phase transition. However, at low temperatures, where most atoms are condensed, $N_{c}$ and $\mu$ can be measured from the size and shape of the observed cloud. A technical disadvantage of in-situ imaging is the need of a very high resolution imaging system allowing to map the tiny sample. The size of some small structures in the condensate wavefunction, e.g. vortices and solitons, may even be beyond the diffraction limit. 


\subsection{Measurements on Condensate Equilibrium Thermodynamics}

The measurement of the temperature dependence of thermodynamical quantities at the phase transition reveals important information about deviations from the ideal gas behavior due to particle interaction, finite size effects and spatial confinement (Chapter 2). Dilute gases are almost ideal systems, the nonideal features are quite small. Therefore they present a better opportunity to study the thermodynamics of Bose phase transitions than other systems, e.g. ${ }^{4} \mathrm{He}$ where the condensed fraction $N_{c} / N$ is difficult to measure and the critical temperature $T_{c}$ is difficult to calculate.

The time-of-flight absorption pictures yield a number of informations. One can extract the total number of atoms $N$ from the missing photons in the probe beam shadow. Two independent two-dimensional Gaussians are fit to the thermal and the condensed part, thus allowing the determination of $N_{c}$. The temperature $T$ of the sample can be estimated by fitting Gaussians to the thermal cloud (outer wings of the total TOF density distribution) assuming thermal equilibrium between thermal and condensed clouds and a Maxwell-Boltzmann velocity distribution for the thermal cloud. The scaling temperature $T_{c}$ is chosen to be the critical temperature (2.23) for an ideal harmonically trapped Bose-gas in the thermodynamic limit (Table 1). Various experiments [6, 120] verified that the condensed fraction $N_{c} / N$ reproduces well the theoretical dependence (2.22) expected for ideal Bose-gases in the thermodynamic limit (Table 11). This means, that the gas is so ideal, that any nonideal gas feature is difficult to be quantitatively measured against the experimental shot-to-shot noise and calibration uncertainties.

However, the measurement of other thermodynamic quantities like the energy and the heat capacity 103, 120] displayed significant deviations from ideal gas behavior and emergence of interaction effects. The same ballistic expansion data set used for determining the condensed fraction can be fit by an arbitrary model-independent density distribution, $\tilde{n}(\mathbf{k})$ if we only make sure, that its zeroth moment is normalized to the atom number $N=\int_{V} \tilde{n}(\mathbf{k}) d^{3} \mathbf{k}$, where $k=m r / \hbar t_{T O F}$. Depending on the chosen distribution, the quality of the fit may be better or worse, but in any case, the second moment gives the kinetic energy, $U=\int_{V} E_{k i n} \tilde{n}(\mathbf{k}) d^{3} \mathbf{k}$, where $E_{k i n}=\hbar^{2} k^{2} / 2 m$. For trapped ideal gases the virial theorem ensures $E=E_{k i n}+E_{p o t}=2 E_{k i n}$, however for real gases the repulsive meanfield energy adds up, $E=E_{k i n}+E_{p o t}+E_{\text {self }}$. The sudden switch-off of the trapping potential nonadiabatically removes the potential energy $E_{\text {pot }}$. The kinetic and the self energy of the BEC are converted into pure kinetic energy during the ballistic expansion. This energy, $E_{k i n}+E_{\text {self }}$, (sometimes called release energy) is measured by TOF measurements. We can expect the temperature dependence of the measured release energy to correspond to the dependence of the total energy. The heat capacity derived from both quantities should give the same results. Despite the experimental noise, the release energy measurement data clearly show a change of slope at the phase transition. Figure 6 shows measurements of the kinetic energy after $10 \mathrm{~ms}$ ballistic expansion.

The measurement was strictly model-independent and contained no assumptions on the quantum statistical nature of the particles or on particle interactions. It would be interesting to compare the results to theories in various limits, i.e. the ideal-gas in the thermodynamic limit or by taking into account finite number effects [39] and particle interactions. However, the accuracy of the experiment does not permit quantitative conclusions. It only shows, that the effect of mean-field repulsion is to increase the energy and to reduce the discontinuity of the temperature-derivative of the energy at the phase transition. 


\section{Chapter 4}

\section{Experiments on Condensate Dynamics}

The experimental achievement of Bose-Einstein condensation in dilute gases triggered a wealth of theoretical and experimental work on the characteristics and dynamics of Bose-condensed gases. The early work focussed on the equilibrium thermodynamics of condensates (discussed in Section 3.4) and their dynamic response to perturbations, especially when they are near the critical point. Since then, breathtaking progress has advanced the field of investigations: Exotic states like vortices, solitons and multispecies condensates have been created, collision resonances have been found, experiments on the interaction of BEC with light have been carried out, and various kinds of atom lasers have been constructed. Chapters 4 to 6 are devoted to giving a brief overview and résumé of recent experiments involving Bose-Einstein condensates. In the present chapter, we focus on experiments on the dynamics of condensates and of binary mixtures of condensates.

\subsection{Wavepacket Dynamics}

The dynamics of Bose-Einstein condensates is generally studied by observing the modification of the shape of the condensate wavepacket in response to time-dependent variations of the trapping potential. The simplest timedependence imaginable is the sudden removal of the trapping potential. Indeed, the first experiment performed on BECs was the study of free expansion [3] (Section 3.3.1). Contrary to the thermal phase which expands isotropically independently of the shape of the trap, the shape of the expanded condensate reflects the trap geometry. The condensed ground state is described by a single macroscopic quantum mechanical wavepacket and expands predominantly into those dimensions originally constrained by the trap. E.g. the BEC aspect ratio reverses during free expansion [90, 117]. Additionally, as the condensate is much denser than the thermal cloud, the condensate self-interaction is much stronger. This repels the atoms and enforces the dynamical evolution described above. Figure 7 shows the evolution of the aspect ratio of a released sodium condensate.

The time-dependence of the trapping potential may also be an oscillatory or pulsed small perturbation, e.g. a tiny modification of the magnetic trapping fields, a pulsed local anisotropy induced by the dipole force of far-detuned laser beams, a manipulation of the condensate self-interaction or density oscillations induced by light coupling to internal or motional degrees of freedom. We will return to these techniques in Section 4.3.

\subsection{Multicomponent Condensates}

\subsubsection{Double Species Condensates in ${ }^{87} \mathrm{Rb}$}

Mixtures of Bose-condensates in different internal states are expected to suffer from relaxation due to spin exchange processes during mixed collisions. Indeed, a collision may scatter atoms into untrapped states or else grant sufficient kinetic energy to eject the atoms from the trap. In the case of rubidium, the fortuitous coincidence between the singlet and triplet ground state scattering lengths reduces the collisional losses for any mixture of spin states. In particular, it allows the coexistence of BECs in the low-field seeking states 
$\left|F, m_{F}\right\rangle=|2,2\rangle$ and $|1,-1\rangle$ 121]. Such double-species condensates have been observed in experiment [7]. When the magnetic trap is loaded with atoms in both hyperfine states, the $|1,-1\rangle$ cloud being less severely confined, extends to larger radii and thus experiences larger magnetic trapping fields. During forced radiofrequency evaporation hot $|1,-1\rangle$ atoms see a lower potential edge than $|2,2\rangle$ atoms and are evaporated faster. If the rate of rethermalizing elastic interspecies collisions is large enough, the $|2,2\rangle$ cloud is cooled sympathetically and simultaneously with the $|1,-1\rangle$ cloud. Under the influence of gravity $|1,-1\rangle$ atoms sag further down into the trapping field than $|2,2\rangle$ atoms, but the displacement is typically smaller than the size of the condensates. Separate imaging of the two BEC components therefore requires appropriate hyperfine-pumping and probing cycles in order to discriminate the two hyperfine states [7].

An alternative method to create two-component BECs has been demonstrated by Matthews et al. 122 . They irradiated a $|1,-1\rangle$ BEC with a microwave-radiofrequency two-photon radiation pulse tuned to the $|2,1\rangle$ state, $\omega_{\text {micro }} / 2 \pi \approx 6.8 \mathrm{GHz}$ and $\omega_{\text {radio }} / 2 \pi \approx 2 \mathrm{MHz}$. With $600 \mathrm{~Hz}$ Rabi-frequency, which is much faster than the secular frequencies of the (uncompressed) trap, $\omega_{\text {trap }} / 2 \pi \approx 100 \mathrm{~Hz}$, they were able to suddenly transfer nearly $100 \%$ of the lower state population to the excited $|2,1\rangle$ state. By transferring only part of the population, they could suddenly spatially mix the two BEC species and study the complicated nonlinear dynamics of spatial reorganization and component separation 123]. The influence of gravity on the TOP trap makes it possible to control the relative vertical displacement of the $|1,-1\rangle$ and $|2,1\rangle$ states by judiciously choosing the values for the trapping field strengths and the TOP rotating frequency [124] and thus allows to realize a considerable overlap region of the two BEC species. The reproducibility of the experimental conditions were good enough to trace the dynamical evolution with destructive time-of-flight imaging by repeating the whole procedure of creating and manipulating the sample. The dynamics is essentially governed by the ratios of scattering lengths between the different components, which have been determined to be $a_{|1,-1\rangle}: a_{|1,-1\rangle|2,1\rangle}: a_{|2,1\rangle}=1.03: 1: 0.97$ [122, 123. Because $a_{|1,-1\rangle|2,1\rangle} / \sqrt{a_{|1,-1\rangle} a_{|2,1\rangle}} \approx 1.0005>1$, the components weakly repel each other. The $|1,-1\rangle$ cloud has a slightly positive buoyancy with respect to the $|2,1\rangle$ cloud.

The coherent coupling of two distinguishable BECs occupying the same region of space has proven a useful tool for several important experiments: The observation of compression oscillations induced by the sudden transfer between the hyperfine states (Section 4.3.1), the creation of vortices in double condensate systems (Section 4.3.4) and the realization of an internal state BEC interferometer (Section 5.3.1).

\subsubsection{Spinor Condensates in ${ }^{23} \mathrm{Na}$}

In order to create a spatial overlap of condensates in different internal states, it is necessary for the atoms to be confined in a common trap and not to suffer from spin relaxation processes. Pure dipole-force optical traps, e.g. red-detuned far-off resonance traps (FORT) are not sensitive to the Zeeman state of the atoms. Therefore, they can trap high field seeking states which cannot be confined in magnetic traps, or even mixtures of atoms being in all Zeeman substates of a hyperfine level. Furthermore, they avoid (spatially inhomogeneous) Zeeman shifts. While attempts to directly produce BECs in optical traps have not been successful yet, $|1,-1\rangle$ sodium condensates have been loaded from a magnetic trap into a FORT [78]. Due to the low kinetic energy of BECs, the optical trap can be made very shallow, so that low power far-detuned lasers may be used. In their experiment, Stamper-Kurn et al. [78] needed only a few milli-Watts of laser power at $985 \mathrm{~nm}$ wavelength focussed down to $6 \mu \mathrm{m}$ waist creating a few micro-Kelvin deep potential to reach trapping lifetimes on the order of $10 \mathrm{~s}$. On the other hand, dipole traps are an order of magnitude steeper than magnetic traps, $\tilde{\omega}_{\text {trap }} / 2 \pi \approx 40-400 \mathrm{~Hz}$. Consequently, very high densities between $n=3 \times 10^{14}$ and $3 \times 10^{15} \mathrm{~cm}^{-3}$, mainly limited by three-body recombination, can be reached.

In a subsequent experiment, Stenger et al. [8] lifted the Zeeman degeneracy by application of a weak magnetic field and coupled the $|1,-1\rangle$ state to the other Zeeman states $|1,0\rangle$ and $|1,1\rangle$ by irradiation of resonant radiofrequency. The population could be completely or partially transferred between the states. The resulting three component BEC quantum field is described by a spinor. Several interesting features of the dynamics of spinor BECs have already been observed, e.g. the formation of spin domains, the miscibility of the $|1,1\rangle$ and $|1,-1\rangle$ and the immiscibility of the $|1, \pm 1\rangle$ and $|1,0\rangle$, the antiferromagnetic behavior of the spin-dependent atomic interaction [8], the metastability of spin domains against very small $(0.1 \mathrm{nK})$ energy barriers [125], and quantum tunneling across spin domains 126] (Section 5.3.2). A review of the experiments on spinor condensates 
can be found in 127]. Spinor BECs are also interesting candidates for studies of nonlinear four-wave mixing processes [28, 128] (Section 5.4.5).

\subsection{Collective Excitations}

\subsubsection{Elementary Excitations}

Elementary excitations (also called quasiparticles or normal modes) of the Bose-Einstein condensate are solutions of the linearized Gross-Pitaevskii equation. The coherent excitation of many quasiparticles leads to collective oscillations or density modulations (also called sound) of the trapped atomic cloud. Technically, the excitations are generated in response to small time-dependent perturbations of the trapping potential. There are various procedures and consequently various types of excitations. The first experiments have been performed by modulating the trapping potential 129, 130 and resulted in the observation of center-of-mass oscillations (also called sloshing modes) and shape oscillations. The frequencies of those excitations agreed well with theoretical calculations [131- 133]. The oscillations were damped by interactions between the collective mode and thermal excitations. The temperature dependence of the damping has been experimentally studied by Jin et al. [134].

At JILA, microwave-radiofrequency double-resonance transitions have been used to suddenly transfer a ${ }^{87} \mathrm{Rb}$ condensate from the $|1,-1\rangle$ into the $|2,2\rangle$ internal atomic state. This state has the same magnetic moment, but a slightly different scattering length, $a_{|1,-1\rangle} \neq a_{|2,1\rangle}$, and therefore a different mean-field energy $g n_{0}=4 \pi \hbar^{2} a_{|2,1\rangle} n_{0} / m$, where $n_{0}$ is the peak density of the condensed cloud. The wavefunction immediately starts to adjust its shape to fit into the modified environment and commences oscillating. These damped compression oscillations have been recorded (Fig. 8) and by comparison to a theoretical model permitted the determination of the ratio of scattering lengths of the two states [122] (Section 4.2.1).

The range of excitations accessible by modulating the magnetic trapping fields is limited. Sophisticated engineering of the perturbation is required to excite more complicated excitations like higher multipolar order surface oscillations [135]. Focussed far-off resonant laser beams are a useful tool to push around the atoms inside a BEC. They have also been used to observe the propagation of sound pulses by generating a short small local density perturbation and tracing its way through the condensate 77]. The temporal behavior of excitations is best studied by taking a rapid sequence of nondestructive images (Section 3.3.2).

The nature of the excitations profoundly depends on their de Broglie wavelengths $k^{-1}=\left(\hbar / 2 m \omega_{k}\right)^{1 / 2}$ compared to three characteristic lengths. Those are the mean free path for quasi-particles $l_{m f p} \approx\left(n_{t h} \sigma_{e l}\right)^{-1}$, the size of the ground state of the trapping potential $a_{\text {trap }}=\left(\hbar / m \omega_{\text {trap }}\right)^{1 / 2}$ and the healing length

$$
\xi=1 / \sqrt{8 \pi a n_{0}}
$$

Here $a=53 a_{B}$ is the sodium $s$-wave scattering length for the $F=1, m_{F}=-1$ state, the cross-section for elastic collisions is $\sigma_{e l} \approx 8 \pi a^{2}$. Typical experimental values for a sodium condensate are $\hbar \omega_{\text {trap }} \approx h 100 \mathrm{~Hz}$ and $g n_{0} \approx h 7 \mathrm{kHz}$, the characteristic lengths are roughly on the order of $l_{m f p} \approx 100 \mu \mathrm{m}, a_{\text {trap }} \approx 2 \mu \mathrm{m}$, and $\xi \approx 0.2 \mu \mathrm{m}$. The mean free path marks the boundary between the hydrodynamic regime, $k^{-1} \gg l_{m f p}$, and the collisionless regime (in the sense of quasiparticle collisions), $k^{-1} \ll l_{m f p}$. The trap size delimits the regime of discrete collective modes, $k^{-1} \gtrsim a_{\text {trap }}$, from the regime of pulsed localized excitations, $k^{-1}<a_{\text {trap }}$. The healing length, finally, sets the boundary between the regimes of phonon-like excitations, $k^{-1} \gg \xi$, and particle-like excitations, $k^{-1} \ll \xi$.

The trap manipulation methods mentioned so far are restricted to the collisionless regimes of discrete collective modes and pulsed localized excitations. The hydrodynamic regime of low energy excitations has barely been approached in experiments [136], and the opposite regime of high energy localized excitations, $k^{-1} \lll a_{\text {trap }}$, has been investigated employing the newly developed method of Bragg spectroscopy [137], [138]. This technique allows to probe excitations over a very large range of frequencies. It is particularly well suited for high frequencies and therefore provides a useful tool to probe the boundary between the regimes of phonon and particle-like excitations. We will return to this in Section 5.4.2. The hierarchy of length scales formed by the various regimes of collective excitations and the experimental method to produce them are summarized in Table 3 . 


\subsubsection{Non-Circulating Topological Modes}

Topological modes are stationary solutions of the Gross-Pitaevskii equation (2.45), that are not the groundstate. There have been some proposals on the creation of such non-groundstate Bose-condensates [139], and an antisymmetric dipole topological mode has recently been created in experiment. Williams et al. 1140, 141] used a coherently coupled double-species condensate. The total order parameter for such a system is a two-dimensional spinor $\Psi(\mathbf{r}, t) \equiv\left(\psi_{|1,-1\rangle}, \psi_{|2,1\rangle}\right)$. We may also view the spinor field as a spatially varying Bloch-vector describing the local internal coherence and inversion of the two-level atoms that form the BEC [141],

$$
\vec{\Psi}(\mathbf{r}, t)=\left(\begin{array}{c}
\psi_{|1,-1\rangle} \psi_{|2,1\rangle}^{*} \\
\psi_{|1,-1\rangle}^{*} \psi_{|2,1\rangle} \\
\left|\psi_{|2,1\rangle}\right|^{2}-\left|\psi_{|1,-1\rangle}\right|^{2}
\end{array}\right) .
$$

The gravitation, whose direction is assumed to coincide with the symmetry axis of the TOP trap, modifies the magnetic trapping potential and displaces the trapped $|1,-1\rangle$ and $|2,1\rangle$ clouds vertically from one another by a tunable amount. When coupling the states with a microwave-radiofrequency two-photon radiation, the axial displacement makes the generalized Rabi-frequency position-dependent, $G(z) \equiv \sqrt{\Omega^{2}+\Delta(z)^{2}}$. The effect of the inhomogeneous coupling strength is a non-uniform precession and nutation speed of the local Bloch-vector and consequently a spatial modulation of the inversion, which comes down to generating a differential torque on each of the single-component wavefunctions. The torque corresponds to a matter-wave phase shift and ultimately reaches a point, where the matter-wave phase twist is $2 \pi$ across the condensate along the $z$-axis. This state is the dipole topological mode. Applying the torque furthermore untwists the phase winding until the condensate finds back to its original state.

In the JILA experiments [141, 142], the time-evolution of the local inversion (third component of the Blochvector (4.2)) and the total space-integrated inversion were monitored non-destructively and on-line by taking sequences of phase-contrast pictures. The probe beam was tuned between the hyperfine states, so that the $|2,1\rangle$ atoms stepped out as rising and the $|1,-1\rangle$ atoms as deepening from the background. The Rabi-flopping of the total space-integrated inversion exhibited a behavior reminiscent to quantum-collapse and -revival well-known to occur in Jaynes-Cummings type systems studied in quantum optics. The epitome of a Jaynes-Cummings system is a two-level atom coherently coupled to a single-mode light field. The driven atom is able to momentarily bury its coherence by transferring it to quantum correlations of the light field. Tracing over the degrees of freedom of the light field, the atomic coherence appears to momentarily collapse and revive at a later time. The coupled system consisting of the BEC matter-wave and the internal atomic degrees of freedom behaves similarly. If all atomic dipoles oscillate in phase (groundstate BEC), the space-integrated inversion exhibits strong Rabioscillations (Figure 9). If the matter-wave field is strongly modulated (twisted condensate, higher topological mode), the atomic dipoles oscillate at different phases, and the Rabi-oscillations of the space-integrated inversion cancel out. Under the influence of the inhomogeneous coupling strength $G(z)$ the system gradually changes its topology and thus causes the collapse and revival of the Rabi-oscillations. A quantitative model can be found in ref. [142].

Matthews et al. [141 extended the experiment later by adjusting the condensate shape for long axial extension, exploiting the tricky TOP-gravitation interplay. The radiation twists the system more and more, successively cranking up to four windings into the BEC. Past some point, the radiation untwists the system again until it finds its way back to the original form.

\subsubsection{Superfluid Flow}

Superfluid liquids or gases are distinguished by their ability to support dissipationless flow, i.e. flow that is exempt from viscous damping. The phenomenon of superfluidity is a well-known property of liquid ${ }^{4} \mathrm{He}$, but the relationship between superfluidity and Bose-Einstein condensation in this strongly interacting system is not trivial. The situation is much simpler in weakly-interacting Bose-gases, where the superfluid phase is nearly identical with the condensed fraction, and the normal fluid phase with the thermal fraction. The availability of dilute gas Bose-condensates offers the unique opportunity to study the superfluid-condensate interdependence. The early experiments on the dynamical behavior of condensates at very low temperatures already provided 
indirect signatures of their superfluid nature, because the hydrodynamic theory of superfluidity describes well the collective excitations, as we have pointed out in Section 4.3.1. Furthermore, the observation of matter-wave interference (Section 5.2.2) is an indication for superfluidity, since quantum coherence is a characteristic of superfluids.

Several experiments provided direct evidence for the superfluid nature of condensates. Raman et al. 143] performed a calorimetric measurement of the dissipation induced by stirring a condensate with a far bluedetuned laser beam. The thermal fraction of the atomic cloud was measured before and after stirring by standard time-of-flight imaging and fitting a bimodal density distribution to the condensed and thermal phases of the cloud (Section 3.3.1). They observed a critical value for the stirring velocity $v_{c}$ : For lower velocities, the perturbation was found to be dissipationfree, at higher velocities phonons were excited and the cloud was heated. In a subsequent experiment, the density fluctuations induced by stirring were observed on-line and in-situ [144. When the stirring velocity was inferior to the critical velocity, the density was quasi stationary at any instant of time thus indicating superfluid flow. However, when the stirring velocity exceeded $v_{c}$, the stirring beam dragged the atoms piling them up in front of it. The resulting pressure gradients led to turbulent flow around the perturbation and dissipation. Taking the asymmetry of the instantaneous density distribution resulting from the bow and the stern wave of the moving laser beam as a measure for the amount of dissipation Onofrio et al. determined a critical velocity that agreed with the result of the calorimetric measurement.

The critical velocity $v_{c}$ found in the stirring experiment was about ten times smaller than the local speed of sound which is inversely proportional to the superfluid healing length (4.1),

$$
c_{s}=\hbar / \sqrt{2} m \xi .
$$

In fact, while the onset of dissipation is accelerated by turbulence around the macroscopic object traversing the superfluid, the local speed of sound (4.3) is derived for a microscopic object. Chikkatur et al. 145] studied the motion of impurity atoms through a condensate. For that purpose, they produced an impurity BEC with well defined initial velocity out of the original BEC by inducing Raman transition from the trapped $\left|F=1, m_{F}=-1\right\rangle$ state to the untrapped $\left|F=1, m_{F}=0\right\rangle$. The initial velocity was set by arranging the Raman laser beams (polarization, encompassed irradiation angle, relative detuning) to satisfy the Bragg condition (Section 5.3.3) and the selection rules for the Raman transition. The impurity, not being constrained by the trap, traversed the BEC before the trap was switched off, a Stern-Gerlach magnetic field gradient was pulsed to spatially separate the atoms being in different hyperfine states and the atoms were probed by time-of-flight imaging. When the initial velocity was well above a critical value that coincided with the local speed of sound, ultracold $s$-wave collisions between the impurity atoms and the stationary condensate distributed the momentum of the collision partners evenly and, in the TOF images (Fig. 10), gave rise to a circular halo centered around the center-of-mass of the collision partners. However, when the initial velocity was reduced, the collision rate between the impurity and the stationary condensate was suppressed and the trajectory was more superfluid.

\subsubsection{Vortices}

Important manifestations of superfluidity are associated with rotational phenomena. An example is the occurrence of scissors modes 146] that are excited when an angular momentum is suddenly applied to an anisotropic BEC. Scissors modes have been generated by Maragó et al. [147]. They produced a BEC in an anisotropic trapping potential and then suddenly reoriented the inclination of the symmetry axis of the trap. The response of the BEC wavefunction is a pure oscillation of its tilt generated by irrotational superfluid flow. The excitation spectrum reflects the strong reduction of the moment of inertia for the superfluid.

The most stringent manifestation of superfluidity, however, is the occurrence of quantized and persistent currents, called vortices. Vortices are stationary solutions (or topological modes) of the Gross-Pitaevskii equation (2.45) that, under the assumption of a cylindrically symmetric system, additionally satisfy the condition $\psi(\mathbf{r})=\psi(r, z) e^{i \kappa \varphi}$, where $\kappa$ is an integer. In a vortex, the superfluid current is driven by the phase gradient, $\mathbf{v}=\hbar / m \nabla \varphi$. The superfluid flow around a close path must by quantized to make the wavefunction single-valued, $\oint \mathbf{v} d \mathbf{r}=2 \pi \hbar / m$, i.e. the phase winds up to multiples of $2 \pi$. Also, the flow must be persistent, because its winding number can only be changed discontinuously by overriding an energy barrier, which requires energy from thermal excitations. The normal component of a gas can have, of course, circular flow, as well. However, the 
disorderly microscopic motion of every individual particle causes a viscous drag that precludes the persistence of the flow in the absence of a driving torque. This is in contrast to superfluid flow which persists even without an externally imposed rotation. Questions about the stability, the formation and the topology of vortices have been addressed in recent experiments [9, 10, 148-150].

Stability. - In a topologically singly-connected trap (e.g. harmonic potential), vortices are not the lowest energy eigenstate, and they must decay into the ground state. If the mean-field interaction energy of the condensate is weak compared to the kinetic energy, $g n_{0} / \hbar \omega_{z} \ll 1$, the healing length is larger than the size of the condensate, $\xi \gg a_{t r a p}$, and the vortex rapidly decays dissipating the excess energy to thermal excitations. Such Bose-condensates cannot be considered superfluid. If the mean-field interaction is strong, the vortex spontaneously breaks the azimuthal symmetry, dislocates from the center and spirals out of the condensate [151. However, the decay time may be pretty long, and under certain conditions a vortex might be trapped off center. On the other hand, a vortex can be the ground-state in multiply-connected traps (e.g. torus-shaped potentials). Such a potential can be realized as the time-average of a harmonic potential with a small rotating anisotropy [10]. Another scheme uses harmonically trapped double-condensates where a ground-state BEC is located at the trap center. An excited internal state BEC can form a vortex in a circular orbit around the ground-state BEC [9]. If the condensates repel each other, the vortex core is pinned by the ground state BEC, so that the vortex is very stable.

Formation. - The ideas on how to create vortices can be divided into two classes. Some propose to imprint an angular momentum into the atomic cloud by rotating the (anisotropic) trap during the process of forced evaporation. This can be done with rotating magnetic fields or by stirring the atomic cloud with a laser beam. When crossing the phase transition, a vortex state should nucleate within the stirring path. Others propose to imprint a circular $2 \pi$ phase gradient into a previously created condensate. These procedures must drive the local density in the center of the trap to zero and then rely on dissipative relaxation of the BEC into the vortex state. Dobrek et al. suggested to exploit the inhomogeneous Stark-shift which a far-detuned optical beam with an appropriately designed intensity profile generates in a BEC [152]. An alternative method based on the phase imprinting idea but avoiding the need of relaxation processes has been suggested by Williams et al. [140. In their configuration the phase gradient is created via adiabatic Raman-transfer between two internal states of the condensate atoms within a small rotating area of space. Thus a coherent process is used to directly build and shape the vortex wavefunction.

The first experimental evidence for vortices was reported by Matthews et al. [9]. They produced and recorded vortices in a coupled double-species condensate system using a method based on the phase imprinting idea. The method consisted in dynamically converting atoms from a nonrotating $|1,-1\rangle$ ground-state BEC to $|2,1\rangle$ atoms (or vice versa) having a torus-shaped topology by time-dependent and spatially inhomogeneous adiabatic population transfer.

In the experiment, Matthews et al. [9] produced a standard $|1,-1\rangle$ ground-state BEC with a size of typically $r_{r m s}=54 \mu \mathrm{m}$ in an isotropic harmonic TOP trap with $\omega_{\text {trap }} / 2 \pi=7.8 \mathrm{~Hz}$ secular frequency. They coherently coupled the two hyperfine states using two-photon microwave radiation tuned $\Delta_{r f} / 2 \pi=94 \mathrm{~Hz}$ below (or above) the resonance and adjusted the radiation power to produce $\Omega_{r f} / 2 \pi=35 \mathrm{~Hz}$ Rabi frequency thus causing the Bloch vector of the two-level system to precess with the generalized Rabi frequency $G_{r f}=\left(\Omega_{r f}{ }^{2}+\Delta_{r f}^{2}\right)^{1 / 2}=$ $2 \pi \times 100 \mathrm{~Hz}$. Spatial and temporal control over the conversion rate between the hyperfine states was achieved by additionally focussing a moveable laser beam $\left(P=10 \mathrm{nW}, w_{0}=180 \mu \mathrm{m}\right)$ onto the cloud and rotating it with frequency $\omega_{\text {rot }}$ at a distance $r_{\text {rot }}=75 \mu \mathrm{m}$ around the symmetry axis of the trap. The laser was detuned $\Delta_{l} / 2 \pi=800 \mathrm{MHz}$ blue from the ${ }^{87} \mathrm{Rb} \mathrm{D} 2$ line thus giving rise to an inhomogeneous time-dependent AC Stark-shift $\Omega_{l}(\mathbf{r}, t)^{2} / 4 \Delta_{l}$, where $\Omega_{l}$ is the Rabi frequency on the $D 2$ transition. While ground-state atoms located at the center of the trap did not sense the modulation of the Stark-shift, atoms located at distance $r_{\text {rot }}$ from the trap center were subject to the full modulation depth and experienced the microwave radiation on two modulation sidebands located at $\Delta_{r f} \pm \omega_{\text {rot }}$. In order to fulfill the resonance condition for one of the sidebands and drive transitions from the ground-state to the $|2,1\rangle$ state, the rotation frequency was adjusted to the precession frequency, $\left|\omega_{r o t}\right|=G_{r f}$. This is equivalent to ensuring, that the phase delay of the precessing two-level Bloch vector, $G_{r f}$, was equal to the azimuthal matter-wave phase variation of the newly created 
$|2,1\rangle$ atoms, $\omega_{\text {rot }} t$, along the rotation path, and that the matter-wave phase was single-valued around a closed loop. The matter-wave phase gradient caused circular flow and formed a visible vortex after about $70 \mathrm{~ms}$. The direction of the vortex rotation could be arbitrarily chosen through the sign of the detuning $\Delta_{r f}$. Vortices could be formed either in the $|1,-1\rangle$ state around a central $|2,1\rangle$ BEC or vice versa. The small positive buoyancy of the state $|1,-1\rangle$ with respect to $|2,1\rangle$ made the first option more stable.

While in harmonically trapped single-species condensates the diameter of the vortex core is on the order of the healing length $2 \xi$ and too small to be seen by in-situ spatial imaging, in the double-species configuration the diameter of the vortex core is much larger, because it is determined by the size of the central core BEC. The core BEC can partially or completely be removed with resonant light pressure and the vortex be studied as a function of the core size and the filling material. To see the vortex, Matthews et al. took a non-destructive image of the density distribution of the upper $|2,1\rangle$ state. Then, on the same sample, they applied a resonant two-photon radiofrequency $\pi / 2$ pulse which mixed the vortex with the core BEC. The resulting ring-shaped matter-wave interference pattern reveals the phase profile of the vortex. This Ramsey type interference technique will be detailed in Section 5.3.1. Finally, a second radiofrequency $\pi / 2$ pulse completely inverted the population of the states $|2,1\rangle$ and $|1,-1\rangle$ and permitted recording the density distribution of the nonrotating ground state (Fig. 11).

Vortex precession. - A radial force acting on a vortex results in its azimuthal displacement and precession around the symmetry axis. The effect is known as Magnus effect 153 and is due to pressure imbalances at the vortex surface. A radial force arises naturally when the core is displaced from the center, because local pressure gradients tend to force it outwards to lower density regions. Anderson et al. 1150 observed a roughly $1 \mathrm{~Hz}$ slow precession of the vortex core by a succession of non-destructive images for various sizes of the core BEC. A radial motion of the vortex as expected for dissipative interaction with a thermal cloud was not observed. Instead, the vortex core decreased its size due to gradually reduced repulsion of the slowly decaying core BEC.

Vortex lattices. - Superfluid ${ }^{4} \mathrm{He}$ in a rotating bucket spontaneously develops patterns of symmetrically arranged vortices. Similar phenomena can be expected when a dilute gas Bose-condensate is forced to rotate. Butts et al. 154 have calculated the vortex patterns that will form as a response to forcing a BEC to rotate with a predefined frequency $\Omega$. The energy in the corotating frame gets an additional contribution from the centrifugal term $U_{\text {rot }}(\mathbf{r})=U_{\text {trap }}(\mathbf{r})-\Omega L_{z}$, where $L_{z}=\hbar N l_{z}$, and $l_{z}=i\left(y \partial_{x}-x \partial_{y}\right)$ is the single-particle angular momentum. If the rotation is slow, the energy $\Omega L_{z}$ is too small to force the condensate wavefunction to rotate. If the rotation frequency is higher than a critical value $\Omega_{c}$, the time-averaged potential, $U_{\text {rot }}(\mathbf{r})$ eventually develops a local maximum in the center (torus shaped potential). For non-interacting gases, the critical frequency coincides with the radial secular frequency, $\Omega_{c}=\omega_{r}$, and the radial restoring force of the trap does not balance the centrifugal force anymore, so that the atoms escape from the trap. However, for superfluid gases the critical frequency is reduced, $\Omega_{c}<\omega_{r}$. Between the rotation frequencies $\Omega=\Omega_{c}$ and $\Omega=\omega_{r}$, the lowest energy state in the torus shaped potential is a vortex filament around the center. At even higher rotation frequencies, one might expect single vortices with a higher winding number (more than $2 \pi$ phase winding for a single path around one vortex). However, single multiple-order vortices in harmonic traps are always very unstable. Instead, vortex lattices 154] are formed. For a given trapping potential and mean-field interaction, the symmetry of the lattice and the number of vortices depend on the rotation frequency $\Omega$. Counterintuitively, the single-particle angular momentum $l_{z}$ is not quantized. Upon varying $\Omega$, forbidden ranges of $l_{z}$ alternate with allowed bands. The discontinuous transition from one vortex pattern to another is a first-order phase transition and spontaneously breaks the previous azimuthal symmetry to form another one. An upper limit for the rotation speed is set by the balance of the centrifugal force and the radial restoring force of the trap at $\Omega=\omega_{r}$.

These vortex patterns have been observed in a recent experiment, that employed the stirring method of rotating the trap [10]. Madison et al. produced a cigar-shaped ${ }^{87} \mathrm{Rb}$ condensate with $N=10^{5}$ atoms in a cloverleaf trap with $\omega_{z}=2 \pi \times 12 \mathrm{~Hz}, \omega_{r}=2 \pi \times 220 \mathrm{~Hz}$. The ratio of mean-field interaction to kinetic energy was $g n_{0} / \hbar \omega_{z}=a_{\text {trap }}^{2} / 2 \xi^{2}>100$. Along the symmetry axis but slightly displaced from the center, they focussed a far red-detuned dipole-force laser beam in order to create a weak anisotropy in the trapping potential. During forced evaporation and while crossing the BEC phase transition, this optical "spoon" is rotated around the symmetry axis. Beyond a certain critical rotation frequency, $\Omega_{c} \approx 2 \pi \times 150 \mathrm{~Hz}$, they observed the formation of 
a central vortex. At higher frequencies, they could image vortex lattices with up to eleven vortices symmetrically arranged in the transversal plane (Fig. 12). At stirring frequencies approaching the radial secular frequency of the trap $\omega_{r}$, the BEC wavefunction got more and more turbulent and finally vanished altogether. After removing the optical spoon, the lifetime of a single vortex was measured to be approximately one second (the lifetime of the condensate being much longer). The vortex decayed to the ground-state of the unperturbed harmonic potential most likely by spiraling out of the center. Vortex patterns were found to decay by successively losing one vortex at a time.

Madison et al. probed the density distribution of the vortices by absorption imaging. The diameter of the dark core of a vortex in the unperturbed trap (no stirring spoon) is set by the healing length and measures about $2 \xi \approx 0.4 \mu \mathrm{m}$. This size is too small for optical imaging. However, after a $30 \mathrm{~ms}$ period of ballistic expansion the core diameter reached $15 \mu \mathrm{m}$ and could be probed easily.

Angular momentum. - Zambelli et al. 155 have suggested a method for measuring the flow around a vortex via the excitation of quadrupolar surface modes. In an axisymmetric trap, the transversal quadrupole modes can be linearly decomposed into two counterrotating modes with angular momentum $\pm 2 \hbar$ and frequencies $\omega_{ \pm}= \pm \omega$. In a rotating BEC, the degeneracy of the frequencies is lifted by an amount that corresponds to the rotational energy of a single atom,

$$
\omega_{+}-\omega_{-}=\frac{2 L_{z}}{m r^{2}}
$$

where $r$ is the average radius of the orbit. This behavior is known as Sagnac effect. Chevy et al. [149] performed an experiment where they first stirred the BEC and then excited the quadrupolar surface modes similarly to the earlier experiment of Onofrio et al. [135]. They observed the quadrupolar oscillation in time-of-flight measurements and noticed a continuous rotation of the principal axis of the quadrupolar mode if vortices had been excited. Also, being in the Thomas-Fermi limit, they could infer $r$ from TOF images and calculate the angular momentum $L_{z}$ of the rotating BEC from equation (4.4) as a function of the chosen stirring velocity. They found $L_{z}=0$ below the critical velocity. At the stirring velocity $\Omega_{c}$, the angular momentum suddenly jumped to $L_{z}=\hbar$ and gradually increased (in fractions of $\hbar$ ) up to $L_{z} \approx 3 \hbar$ as the stirring was further accelerated. At stirring velocities approaching the radial trap frequency $\omega_{r}$, the vortex pattern got turbulent and $L_{z}$ diminished again and finally vanished.

\subsubsection{Matter-Wave Solitons}

Solitons are localized non-singular solutions of any nonlinear wave equation satisfying $|\psi(\mathbf{r}, t)|=|\psi(\mathbf{r}-\mathbf{v} t)|$. Solitons are well known to occur in nonlinear optical media, e.g. in optical fibers when the dispersion is counterbalanced by self-phase modulation so that they propagate without spreading. The Gross-Pitaevskii equation is another example of a nonlinear wave equation that can exhibit soliton-like solutions. Correspondingly, so called dark solitons or kink-wise states, i.e. states with dynamically stable density minima, are expected in condensates with repulsive interactions. They have been predicted for one-dimensional BECs 156]-159] and may occur in higher dimensions, as well. In contrast to truly topologically stabilized defect states like vortices, dark solitons are pseudodefects, whose decay may be very slow although they are topologically trivial. Due to the greater motional freedom of their wavefunctions they may be untwisted by complex deformations 160 . Matter-wave soliton-like states have first been observed in superfluid ${ }^{3} \mathrm{He}-\mathrm{B}$ [161]. In dilute gases, their size is expected to be on the order of the healing length which typically corresponds to a few hundred nanometers.

Dum et al. 162 proposed to engineer dark solitons in Bose-condensates using adiabatic Raman-transfer, and many other schemes have been suggested. Burger et al. [163] and the NIST group at Gaithersburg [164] recently successfully created and observed solitons. Both groups employed a method based on the application of an inhomogeneous matter-wave phase-shift. They created and magnetically confined a rubidium resp. sodium condensate and irradiated half of the condensate with a far-off resonance laser beam pulse (detuning $\Delta$, Rabi frequency $\Omega$, duration $\left.\tau \ll \hbar / g n_{0}\right)$ thus advancing the phase of this half condensate by $\varphi=\Omega^{2} \tau / 4 \Delta$. When the phase-shift was adjusted to be on the order of $\pi$, a steep phase gradient developed at the boundary plane driving the density distribution in the condensate to adjust itself until a density minimum formed along the plane. The density distribution of the condensate was mapped by time-of-flight imaging at various delays after 
application of the phase shifting laser pulses (Fig. 13). Denschlag et al. additionally used an interferometric technique based on Bragg diffraction (Section 5.3.3) to monitor the phase distribution of the condensate. The observed density kink corresponds to the node of a topological dipole mode and can also be interpreted as a one-dimensional dark soliton on a finite background, where the kink and the background move synchronously [140, 156, 159]. These states represent standing matter-waves for which the trap serves as a cavity.

The steep phase gradient at the nodal plane exerts a force that tries to enhance the gap, while the repulsive interaction works to fill it. At zero temperature, this balance guarantees the dynamical stability of the soliton. While a perfectly dark soliton should be stationary, the experiments 163, 164 exhibited a propagation of the density kink perpendicularly to the nodal plane. This feature is a result of the finite contrast of the kink. However, the propagation velocity must always be inferior to the local speed of sound,

$$
v_{s o l}=c_{s} \sqrt{\frac{n_{s o l}}{n}}
$$

where $n$ is the condensate peak density and $n_{\text {sol }}$ the density at the bottom of the dark soliton 157, 158. Figure 13 shows that the soliton develops a curvature as it propagates. The reason for this is the decrease of the local speed of sound, $c_{s}=\sqrt{g n_{0} / m}$, at the edge of the BEC where the density gets smaller. A second reason is that the density in the dip $n_{\text {sol }}$ tends to zero towards the edge. In the presence of a thermal cloud, dissipation reduces the contrast of the density kink and accelerates the soliton until it reaches the speed of sound $c_{s}$ and finally vanishes. 


\section{Chapter 5}

\section{Atom Optics with Bose-Einstein Condensates}

In the past decade, various methods and schemes of laser cooling and trapping of atoms became powerful tools in atom physics and quantum optics. In achieving always lower temperatures and extreme densities, the whole field moved to the boundaries of the new regime, where coherent matter-wave interactions become dominant. This development culminated in the experimental achievement of Bose-Einstein condensation. The atoms confined on microscopic or macroscopic scales at high phase space density are governed by collective and quantum statistical effects. This opens up new perspectives for many-body studies in regimes, where standard approximations cease to be valid. It is, for example, particularly interesting to explore atomic two-body interactions, which may play a role in coherent matter-wave optics similar to the role played by atom-photon interactions in quantum and nonlinear optics. At the same time, the field of atom optics developed rapidly with the demonstration of atom optical elements like atom mirrors, atom lenses and beamsplitters. These two fields are now being combined and form the basis of the new emerging field of coherent atom optics.

The atom laser is the matter-wave analogue of the photon laser. It is a coherent atom source "pumped" by an ultracold and dense ensemble, which is stimulated to feed one mode of the atomic de Broglie field. A Bose-condensate trapped in the ground state of a confining potential and fed from an evaporatively cooled thermal cloud may already be understood as a rudimentary stationary pulsed atom laser. The trap plays the role of the laser cavity. However, while lasers can oscillate in any cavity mode, BECs generally condense in the ground state of the trap. Similar to the invention of the laser in the early sixties with all its fascinating scientific applications, coherent atom sources will open new areas of fundamental physics and applications, in part still unforeseen. These may include atom interferometry, atom lithography, atom microscopy, atom holography, atom sensoring or nanostructuring. Many applications demand dense, bright and coherent sources of atoms in order to exhaust their capabilities. In this respect, atom lasers are much superior to thermal atomic beams. While a typical thermal beam has about $10^{-12}$ atoms per mode, a Bose-condensed mode contains $\gg 1$ atoms. The recent demonstrations of the experimental feasibility of Bose-Einstein condensation [3]- [6], [110, 165] boosted theoretical and experimental work and accelerated the development of this whole field.

As an example, atomic holography may become practical with the availability of spatially coherent matterwave sources. Microfabricated holograms may have typical dimensions of a few $100 \mu \mathrm{m}$ and minimum feature sizes on the order of $1 \mu \mathrm{m}$. Exploiting the repulsive self interaction, one may let a BEC expand, pass it through a transmission hologram computed judiciously to produce the desired diffraction pattern, and refocus it. Small chromatic aberration is due to small velocity spread. The resolution is limited on one hand by the atomic de Broglie wavelength, on the other hand by the size of the smallest structures of the hologram [28, 166, 167] which can be made as small as $10 \mathrm{~nm}$. Another important quantity, the resolving power, is limited by the number of holes in the hologram and the velocity spread of the atoms. While the reduction of the velocity spread by spatial filtering of an incoherent atomic beam is only possible at the cost of huge loss in intensity, the velocity spread of coherent matter-waves is at its quantum limits.

The present chapter reviews recent experimental work on coherent atom optics. In order to place our topic 
into the right context, we start with a very brief overview of conventional atom optics. We discuss the impact of the advent of BEC on the field of atom optics in the Sections 5.2 and 5.3, on the basis of recent realizations of atom lasers and interferometers. Section 5.4 is devoted to experiments in nonlinear atom optics, Section 5.5 relates the recent demonstration of a coherent matter-wave amplifier and gives a brief outlook on the evolution of the field of quantum optics with atoms.

\subsection{Conventional Atom Optics}

The far-reaching analogy of light waves and atomic beams is a result of the particle-wave duality and thus of the quantum nature of both light and matter. It motivated de Broglie in 1924 to assign a wavelength to material particles that depends on the particle's momentum:

$$
\lambda_{p}=\frac{\hbar}{p} .
$$

Contrary to photons [168, there is no doubt about the fact that atoms are (also) particles. Whether an atom rather behaves like a particle or a wave depends on the specific experimental situation. In interferometers, atoms interfere with themselves if their de Broglie wavelength is coherently split and recombined. Atoms are capable of interfering with one another if their de Broglie wavelength is larger than their distance. This requires high densities and very low temperatures (at least in some dimensions). In fact, what matters is not the small kinetic energy of the atoms, but a small velocity spread, i.e. a high phase-space density. At phase-space densities so high that the atomic de Broglie waves get into contact, quantum statistical effects start to influence the atomic dynamics, i.e. Bosons behave differently from Fermions.

Analogously to the distinction between classical optics and laser optics, we may divide the field of atom optics into conventional single-atom optics with atoms that are not mutually coherent and atom optics with Bose-condensed atoms. In conventional atom interferometers, one takes advantage of the interference of every atom with itself, and most atom optical devices do not rely on the mutual coherence of the atoms. On the other hand, nonlinear interactions between the atoms make the dynamics of coherent matter-waves interacting with atom optical devices much more complex than single-atom optics. Atom optics with condensates offers the advantage of large de Broglie wave amplitudes and ultra-long de Broglie wavelengths. In fact, the coherence length of a BEC is equal to its physical size. This has obviously an important impact on the sensitivity and resolution of atom optical devices, as we shall soon see.

We will not go into details about conventional (single-particle) atom optics here, since there are many excellent topical reviews [169]-172. However, for the sake of completeness, we list below the most important atom optical devices that have been developed and used in experiments.

\subsubsection{Atom Optical Devices}

In analogy to the manipulation of light beams by optical elements, atom-optical components have been developed for manipulating atomic matter-waves. The basic equipment of an optics lab consists of cavities, lenses, refractive, dispersive and birefringent media, mirrors, beamsplitters, transmission and reflection gratings, fibers, acousto-optic modulators 169, 173. The matter-wave counterparts of all those elements have been realized today. Most elements exploit the interaction of the mechanical degrees of freedom of the atoms with light. Note that the atom optical devices only manipulate the atomic field density and the first order coherence.

Atomic beams have a long history of applications in ultra-high precision experiments, e.g. in atomic clocks [174. Since the development of laser cooling techniques, atomic beam slowing and cooling has proven a powerful source for many applications 64, 65. Today, atomic beams are often used to load magnetic, optical, and magneto-optical traps for atoms.

Traps for atoms are to some extent analogous to optical cavities for light. In second quantization the radiation field inside a cavity is described by harmonic oscillators, just like the motion of atoms confined in a harmonic trap 175. At very low temperatures, $k_{B} T<\hbar \omega_{\text {trap }}$, the effects of quantized motion can be directly observed [74, 176]. 
Lenses for atomic waves may be realized by exploiting the radiation forces of laser beams, or if the atoms are moving within a waveguide, by arranging for spatial or temporal variations of the fields 177] - 179]. Mirrors for atoms can be made by a far blue-detuned evanescent wave emerging from the surface of a glass substrate 180 . Aspect and his group let atoms bounce in the gravitational field on a curved and (for matter-waves) achromatic mirror more then twenty times 181. This is a rudiment for a gravitational cavity for atoms, where many atomic bosons could occupy one cavity mode in analogy to optical resonators. Another option for atomic mirrors is a microfabricated magnetic surface that repels the atoms approaching the strongly inhomogeneous magnetic surface field [182]. Already, falling Bose-Einstein condensates have been reflected from a far blue-detuned sheet of light [183]. Gratings are microfabricated [184] or based on standing light waves. They are at the heart of atom interferometers and already permitted the development of high precision applications and experiments (atomic gyroscopes, measurement of the gravitational acceleration $g$ ). Waveguides are the atom-optical counterpart of fibers. Forces that guide the atoms can be exerted by electric or magnetic fields (single wire [185], quadrupolar waveguides [186]), or by light beams via the dipole interaction. Possible geometries are evanescent wave hollow fibers [187, 188 or blue-detuned hollow-core laser beams [189. Recently, Bose-Einstein condensates have been transferred to such hollow-core laser beam waveguides 190]. Inhomogeneous magnetic fields (e.g. magnetic trapping fields) act as Stern-Gerlach filters and can be thought of as matter-wave polarizers.

De Broglie wave frequency shifters are the matter-wave analogue of acousto-optical modulators (AOM). They have been implemented in conventional atom interferometers 191 and more recently in coherent matter-wave optics [20]. The matter-wave experiment will be discussed in Section 5.3.3. Finally, we want to mention atom holography [167], atom lithography [177] and atom microscopy [192] as examples for the successful application of matter-wave optics. It is very likely that the availability of coherent matter-waves will have a strong impact on these fields, too.

\subsubsection{Atom Interferometers}

Atom interferometers split and recombine a single atom or an atomic ensemble in time or in space (or both). If the temporal or spatial evolution is coherent, we observe interference phenomena. In many experiments one attempts to produce large splittings of the atomic de Broglie wave, but even a motionless single particle can act as an interferometer and produce Rabi- or even Ramsey fringes [193]. Recoil effects in the interaction of atoms with light become important when the atoms are so cold that the atomic momentum verges on the wavevector of the photons, $\mathbf{p} \approx \hbar \mathbf{k}$. Each absorbed photon adds a quantized amount of momentum, $\hbar \mathbf{k}$, to the motion. The application of light-induced $\pi / 2$ pulses to the atoms splits the de Broglie wave and entangles the internal and motional degrees of freedom. Variations of this idea led to the development of Ramsey-Bordé interferometers and atomic fountains 194 .

\subsection{Atom Laser}

Probably the most striking feature of Bose-condensed atoms is their mutual (first-order) coherence spectacularly demonstrated by Andrews et al. [195|. In quantum optics, the epitome of a coherent light source is the laser, and we may ask, whether there is a matter-wave analogon, and what the relationship between such an atom laser and a Bose-condensate would be. In fact, we may already consider a BEC to be a rudimentary stationary atom laser pulse, the trapping potential taking over the role of the cavity. The atom laser, in the sense of a coherent atomic wave emitting device, must satisfy a few more requirements. Generally, we ask for a continuously working output coupler for a coherent atomic beam and an irreversible pump process that refills the atom-lasing medium. Many theories on atom lasers or bosers have been developed [196-204, and we will not explain them here. Instead, along the lines set by the analogy between optical and conventional atom optical devices, we will briefly describe the experimental progress that has been made on the way towards an atom laser that deserves this name. 


\subsubsection{Bosonic Stimulation and Evaporation}

The gain mechanism for optical lasers can be understood as photons in a laser mode stimulating atoms to emit more photons into the same laser mode. The atom laser works similarly. The atoms trapped in a potential constitute a thermal reservoir. Binary collisions redistribute the atoms over the energy states. If a state already contains a population of $N$ atoms, the Bose quantum statistics encourages an atom involved in a collision process to join this state. The bosonic enhancement factor is proportional to $N+1$. Bose-condensation is necessarily a result of bosonic stimulation. However, the dynamics and the time scale of the formation process were controversially discussed, until recent experiments performed at the MIT clearly demonstrated that BECs form at finite times and develop long-range order.

In order to directly observe bosonic stimulation, Miesner et al. [205] evaporatively cooled $2 \times 10^{7}$ magnetically trapped atoms close to the condensation threshold at $1.5 \mu \mathrm{K}$. The final temperature was set by the final $r f$ frequency of the evaporation ramp. Then they suddenly decreased the $r f$ frequency by $200 \mathrm{kHz}$, thus initiating a fast truncation of the hot tail of the energy distribution. The quick subsequent relaxation produced an oversaturated "thermal" cloud, and the nucleation process and exponential growth of the BEC within the thermal cloud was observed time-resolved by non-destructive dispersive imaging of the atomic cloud (see Section 3.3.2).

Figure 14 shows the growth of the condensate atom numbers towards equilibrium starting with various condensed atom numbers at the time of the fast $r f$ truncation. If no condensate was present, the growth started slowly and increased exponentially until thermal equilibrium was reached. The exponential acceleration of the growth is a clear indication of bosonic stimulation and is in contrast to pure thermal relaxation, which slows down exponentially. For the experimental conditions (the trap secular frequencies were $\omega_{r}=2 \pi \times 83 \mathrm{~Hz}$ and $\omega_{z}=2 \pi \times 18 \mathrm{~Hz}$ ) the formation of BEC took about $40 \mathrm{~ms}$, while elastic collisions happened on the time scale of $2 \mathrm{~ms}$. The large collision rate ensured that during the process of forced radio-frequency evaporation (Section 3.1.4) the atomic sample is always held in thermal equilibrium so that, even while crossing the phase transition to $\mathrm{BEC}$, the condensed fraction of atoms reflects the instantaneous temperature rather than the dynamics of condensate formation.

Other experiments have confirmed the role of bosonic stimulation and matter-wave amplification. They will be discussed in Section 5.4.4 and 5.5.2.

\subsubsection{Coherence and Interference}

First-order coherence and long-range order are necessary prerequisites for the assignment of a single global phase to a condensate. The coherence properties of BECs and the possibility to measure a condensate's phase have in the past been questioned. The phase of a BEC is certainly not observable by itself, but only the relative phase of two condensates. In superconductors, phase-differences between the order parameters of coupled systems are measured through Josephson-oscillations. For dilute gases, the first-order coherence and long-range order of the condensate wavefunction have been demonstrated in a remarkable experiment by observing matter-wave interference fringes generated by two overlapping condensates [195].

Andrews et al. 1195 produced a cigar-shaped BEC made of $5 \times 10^{6}$ atoms and no apparent thermal cloud in a cloverleaf trap with secular frequencies $\omega_{r} \approx 140 \cdot \omega_{z} \approx 2 \pi \times 243 \mathrm{~Hz}$. They subsequently cut it into two parts distributed along the weak axis with a $12 \mu \mathrm{m}$ thin laser light sheet (Fig. 15). The laser light was blue-detuned by $75 \mathrm{~nm}$ below the $D 2$ resonance, so that heating due to Rayleigh scattering could be neglected. The two parts of the condensate were then released from the trap by suddenly removing all magnetic fields and laser beams. During free expansion, the condensates progressively overlapped and formed interference fringes. After $40 \mathrm{~ms}$ time-of-flight, the interference patterns were probed by absorption imaging (Section 3.3.1). Standard absorption techniques only sense the integrated column density and blur the images of the slightly curved interference patterns (Fig. 16] 206. Andrews et al. solved this problem by only probing atoms within a $100 \mu \mathrm{m}$ thin slice orthogonal to the imaging direction. This was achieved by selectively pumping the atoms within this slice to the $F=2$ hyperfine level of the groundstate which in turn is resonant to the probing transition.

Two condensates interpenetrating at a velocity $v$ exhibit interference fringes with a periodicity that corresponds to the relative de Broglie wavelength $\lambda=h / m v$, where $v=d / t$. Here, $t$ is the time-of-flight and $d$ 
is the initial separation of the BECs assumed to be ideal point sources, but the finite extension makes only small modifications. The interference patterns observed in experiment [195] depended on the initial separation of the condensates, on the time-of-flight and on the way they were released from the trap (pulsed or cw). The interference fringe contrast was found to be between $50 \%$ and $100 \%$. The interference fringes periodicity was on the order of a few micrometers, which corresponds to atoms having a kinetic energy much lower than the mean-field energy and the zero-point energy of the harmonic trap. The reason for this is the large anisotropy of the trapping potential: The released condensates expand predominantly in radial direction, but are very slow in axial direction.

The ability of freely expanding condensates to interfere proves that there are no random local phase-shifts during ballistic expansion, and that the BECs preserve their long range order. The homogeneity of the intrinsic phase of trapped BECs has recently been confirmed in other experiments 137, 207, 208 (Section 5.4.2) based on the technique of Bragg diffraction (Section 5.3.3). Simsarian et al. 209 measured the evolution of the local phase of released condensates and found that, under the influence of mean-field repulsion, the phase develops a non-uniform profile during the ballistic expansion.

In the original interference experiment [195], the magnetic trapping fields created in conjunction with the laser light sheet a double-well potential. However, the potential well was so large, that it prevented tunneling between the condensates and decoupled their dynamics. Different atom numbers in the condensates, imperfections in the exact symmetry of the two traps and technical noise caused the condensate phases to evolve independently and asynchronously. However, it might be possible in future experiments (e.g. by employing a very narrow light sheet) to allow for quantum tunneling and, ultimately, to observe Josephson oscillation between two condensates (Section 5.3.2).

The degree of coherence (i.e. the amount of fluctuations in the field amplitudes) is measured by the firstorder correlation function. Similar to optical double-slit experiments, the observed matter-wave interference only indicates first-order coherence of the interfering beams. However, signatures for higher-order short range coherence of condensates have been found in other experiments: The second-order correlation function, which is a measure of the amount of fluctuations in the field intensities, has been estimated from measurements of the release energy of BECs [210]. The third-order correlation function revealed itself by comparison of the three-body recombination rates of condensed and thermal clouds [211].

\subsubsection{Output Coupling}

Coherent output coupling from Bose-condensates out of magnetic traps is generally achieved by radiatively coupling trapped $|-\rangle$ and untrapped $|+\rangle$ Zeeman-states. A condensate of $N$ atoms driven with Rabi frequency $\Omega$ evolves with time $\tau$ into a superposition $\left[b_{-}|-\rangle+b_{+}|+\rangle\right]^{N}=\sum_{n=0}^{N} \sqrt{\left(\begin{array}{c}N \\ n\end{array}\right)} b_{-}^{N-n} b_{+}^{n}|N-n, n\rangle$, with $b_{-}=\cos \Omega \tau / 2$ and $b_{+}=\sin \Omega \tau / 2$. The total wavefunction describes an entanglement between trapped and untrapped states, $|N-n, n\rangle \equiv \sqrt{\left(\begin{array}{c}N \\ n\end{array}\right)}|-\rangle^{N-n}|+\rangle^{N}$, which is analog to the coherent splitting of a photon Fock state by an optical beamsplitter. The inhomogeneous trapping potential acts like a Stern-Gerlach filter and ejects the untrapped atoms. We have, however, seen in the previous section, that the release process preserves the intrinsic coherence of the released BEC, which propagates according to a single-mode wave equation. The coupling between trapped and untrapped condensates therefore remains truly coherent.

Coherent output coupling of parts of condensates out of magnetic traps has been realized in several different ways. Radiofrequency radiation was used for pulsed [14, 18] and continuous [16] output coupling. Laser beams in Raman configuration have been used to create a quasi-continuous, well-collimated coherent atomic beam [17, and a mode-locked system has been demonstrated [15]. In this section, we will briefly discuss the experiment of Mewes et al. 114.

The first output coupling experiment was performed by Mewes et al. in a sodium condensate with $5 \times 10^{6}$ atoms and no discernible thermal fraction confined in a cigar-shaped cloverleaf trap with secular frequencies $\omega_{r} \approx 20 \omega_{z} \approx 2 \pi \times 400 \mathrm{~Hz}$. The magnetic trapping field had a bias of $B_{0}=1.1 \mathrm{G}$, which removed the degeneracy of the trapped $m_{F}=-1$ and untrapped $m_{F}=0,1$ Zeeman states within the lower hyperfine multiplet $F=1$. Mewes et al. coupled them via radiofrequency radiation. With time, the system evolved into a coherent superposition of Zeeman states $\left[b_{-1}|-1\rangle+b_{0}|0\rangle+b_{1}|1\rangle\right]^{N}$, where $b_{-1}=\cos ^{2} \Omega \tau / 2$ and $b_{0}=i \sqrt{2} \sin \Omega \tau$ and 
$b_{1}=-\sin ^{2} \Omega \tau / 2$. Atoms in the $m_{F}=1$ state were quickly repelled from the trap center by the magnetic field, while atoms in the $m_{F}=0$ state were slowly accelerated by gravity. The spatial dependence of the Zeeman shift in the magnetic trap inhomogeneously broadened the radiofrequency resonance and made the output coupling efficiency spatially dependent. This problem was solved by either sweeping the radiofrequency through the resonance or by applying pulses so short, that the Fourier broadening dominated the inhomogeneous broadening. Repetitive application of $5 \mu$ s long pulses gave rise to the absorption images shown in Figure 17 . By controlling the amplitude of the radiofrequency, the output coupling could be adjusted between $0 \%$ and $100 \%$. In a subsequent experiment, it was verified that the output coupling preserves the coherence by observing interference fringes between outcoupled pulses [195]. This also shows that this output coupler may be understood as the analogue of a pulsed mode-locked laser.

It is also important to consider collisions between the output coupled atoms and the atoms remaining in the condensate. Those collisions represent losses for the output mode and may even lead to bosonically fed momentum sidemodes (Section 5.4.4). A low condensate density is advantageous for reducing the atomic scattering. On the other hand, the BEC gets superfluid at high densities thus allowing the dissipationless motion of the output coupled atoms through the BEC. We remind here of the study of the motion of impurity atoms through a condensate by Chikkatur et al. [145].

Within the small region of space occupied by the trapped condensate, the magnetic field is harmonic to first order except for a tiny deformation at the bottom side due to gravity. For the experiment described above, the deformation corresponded to about $10 \mathrm{mG}$ magnetic field variation. Precise tuning of the radiofrequency to this value results in a spout through which slow atoms may continuously escape, thus generating continuous and precisely localized output coupling. Of course this method requires very stable magnetic fields. This method has been used by [16], to create a quasi-continuous atom laser beam.

We have seen, that atom lasers can be built including all features that make up an optical laser. We can generate coherent matter-waves taking atoms from a thermal reservoir by irreversible bosonically stimulated scattering, and we can couple (quasi-)continuous coherent atomic beams out of a single mode of the trap. However, the mode only contains a finite number of condensed atoms. In order to realize a true cw atom laser, an incoherent pump mechanism that would continuously refill the BEC being depleted by output coupling still remains to be developed.

\subsection{Atom Interferometry}

The most obvious use of an atom laser is within an atom interferometer. We already saw in Section 5.2.2 that we get matter-wave interference by just splitting and recombining a Bose-condensate. Andrews' experiment 195 thus realizes an external degree of freedom coherent matter-wave interferometer, where the atoms in the interferometer arms are distinguished by their being at different locations. It is also possible to build an interferometer based on splitting the BEC in momentum space as we will see in Section 5.3.3 [20]. Alternatively, we may consider BEC atom interferometers, where the interfering components are in different internal states (Zeeman-states [8], hyperfine states [7], dressed states [212]). We will briefly discuss an experimental implementation of an internal state BEC interferometer in the following section.

\subsubsection{Double Species Interferometer and Phase Measurements}

The possibility to coherently couple two-species Bose-condensates, i.e. two BECs that are distinguishable by their internal degrees of freedom, suggests their application on an internal-state time-domain atom interferometer [193]. The phases of the two internal states $| \pm\rangle$ evolve according to their respective chemical potential, $\varphi_{| \pm\rangle}(t)=$ $\mu_{| \pm\rangle} t$. The phases are not observable, but their difference $\Delta \varphi(t)$ can be measured by Ramsey interferometry. The idea of a Ramsey interferometer is the following: First, a coherent superposition of the internal states is created by coupling the two internal states for a short time. The two-level Bloch vector then starts to precess according to the difference in the chemical potentials. After a while, the internal states are mixed again, and the Bloch vector is projected onto the internal state energy axis. The population distribution between the internal states 
depends on the accumulated precession angle. Thus, the Ramsey method of separated oscillatory fields converts the phase measurement into a measurement of populations, which can easily be carried out experimentally.

Hall et al. [19] started with a single $|1,-1\rangle$ BEC having a well-defined global phase. A first two-photon microwave-radiofrequency pulse prepared a coherent superposition of $|1,-1\rangle$ and $|2,1\rangle$ BECs. With a resonant $\pi / 2$ pulse, they got $50 \%$ population in both levels. The converted and the remaining atoms were not immediately in the stationary ground-states of their respective trapping potentials, because they had to adjust the spatial shape of their condensate wavefunction to the modified conditions. The smaller partial atom numbers, a slight change of the scattering length and of the trapping potentials for the atoms turned into $|2,1\rangle$ altered the chemical potentials for both states. So, it took some time for the two BECs to relax to their respective ground states. During a time $T$, the two-level Bloch vector freely precessed, and the BECs accumulated a differential phase proportional to the difference in their chemical potentials. A second $\pi / 2$ pulse now remixed the components. Finally, the populations in $|1,-1\rangle$ and $|2,1\rangle$ were separately probed via time-of-flight imaging.

The TOP trap offers the possibility to precisely tune the relative displacement of the two clouds. The interpenetration can be made considerable and typically amounts to $20 \%$. The overlap region constitutes the interfering portion of the BEC interferometer, and its size determines the fringe visibility. A simple model describes the fringe contrast as a function of the local densities $n_{\left|F,-m_{F}\right\rangle}$. The final population of the $|2,1\rangle$ state after completion of the Ramsey sequence reads [19]:

$$
n_{|2,1\rangle}^{f}(\mathbf{r})=\frac{1}{2} n_{|1,-1\rangle}(\mathbf{r})+\frac{1}{2} n_{|2,1\rangle}(\mathbf{r})+\sqrt{n_{|1,-1\rangle}(\mathbf{r}) n_{|2,1\rangle}(\mathbf{r})} \cos \left[\int_{0}^{T} \Delta \mu(\mathbf{r}, t) d t\right] .
$$

Ramsey fringes were recorded [19] by repeating the whole sequence of BEC creation, Ramsey interferometry and destructive imaging with different free precession times $T$. In equation (5.2), we assumed an inhomogeneous and time-dependent evolution of the local relative phase at a rate proportional to the local difference in chemical potentials $\Delta \mu(\mathbf{r}, t)$. This assumption accounts for the complicated transient relaxation of the two partial condensates into their respective ground-states. The transients should lead to phase diffusion in the spatial average and engender strong decoherence. However, in experiment [19] the fringes turned out to be surprisingly clear and reproducible, thus indicating lower phase diffusion than naively expected. The relaxation typically lasted $45 \mathrm{~ms}$, but even after $T=100 \mathrm{~ms}$ the double BEC system remembered the initial phases and could interfere. Furthermore, $\Delta \mu(\mathbf{r}, t)$ depends on the numbers of atoms in the upper and lower BECs and therefore on the total condensed atom numbers. The high fringe visibility indicates a very good experimental reproducibility.

Note that phase and atomnumber are non-commuting observables. Measuring the difference in atomnumbers of two coupled Bose-condensates destroys the relative coherence and decouples the BECs. Internal coherence of a BEC means predictable phase between any two atoms. Atomnumber measurements yield BEC number states, but of course this does not diminish the inherent coherence of the BEC.

\subsubsection{Quantum Transport and Josephson Tunneling}

When two superconductors are brought into contact, a $d c$ voltage that is applied to a tunnel junction between the superconductors generates an oscillating current proportional to the electric potential difference. This phenomenon, called Josephson effect [213], is a general feature of coupled macroscopic quantum systems and can be observed e.g. with gaseous Bose-condensates confined in a double-well potential. Here, the oscillating quantum current is proportional to the difference in chemical potentials of the BECs.

Anderson et al. [15] have directly observed another manifestation of Josephson tunneling. They loaded a Bose-condensate into a vertical standing light wave. Accelerated by gravity, the BEC tunneled from antinode to antinode. Since the tunneling process was coherent, the partial BECs quasi-trapped in the antinodes were phase-locked and interfered. This feature is in close analogy to mode-locked lasers, so that the tunnel array can also be considered a mode-locked atom laser.

Another example for coherent matter-wave tunneling is the experiment by Stamper-Kurn et al. [126] on spinor BECs trapped in a focussed far-detuned laser beam (Section 4.2.2). In a weak magnetic bias field, the BEC was transferred into a superposition of the spinor components $\left|F, m_{F}\right\rangle=|1,1\rangle$ and $|1,0\rangle$, which were then 
separated with a Stern-Gerlach type magnetic field gradient and formed spin domains. Then the magnetic field gradient was reversed, thus generating a force in the opposite direction. Since the Zeeman components are immiscible, the domains repel each other. The energy barrier is higher than the chemical potential of the domains. This means that the domains are metastable against decay into their respective equilibrium positions. The experiment [126] observed quantum tunneling of the spinor components through each other and measured the tunneling rate.

\subsubsection{Bragg Diffraction}

We now turn our attention again to the external degrees of freedom of the Bose-condensed atoms and take a closer look at the interaction of their center-of-mass motion with light. We consider two laser beams with wavenumbers $k_{\omega}=\omega / c$ and $k_{\omega+\Delta \omega}$ enclosing an angle $\vartheta$ (Fig. 18). The light field amplitude is described by

$$
E(\mathbf{r}, \mathbf{t})=E_{0}\left[\cos \left(\mathbf{k}_{\boldsymbol{\omega}} \cdot \mathbf{r}-\omega t\right)+\cos \left(\mathbf{k}_{\boldsymbol{\omega}+\boldsymbol{\Delta} \boldsymbol{\omega}} \cdot \mathbf{r}-(\omega+\Delta \omega) t\right)\right] \approx E_{0} \cos \left(\mathbf{k}_{\boldsymbol{\omega}} \cdot \mathbf{r}-\boldsymbol{\omega} t\right) \cos \left(\frac{1}{2} \mathbf{q} \cdot \mathbf{r}-\frac{1}{2} \Delta \omega t\right),
$$

where we defined $\mathbf{q} \equiv \hbar \mathbf{k}_{\omega}-\hbar \mathbf{k}_{\omega+\Delta \omega}$. The time-average over an oscillation period yields the light intensity

$$
I(\mathbf{r}, \mathbf{t})=I_{0}[1+\cos (\mathbf{q} \cdot \mathbf{r}-\Delta \omega t)]
$$

which describes a one-dimensional light grating moving in the direction of $\mathbf{q}$ with a velocity that depends on $\Delta \omega$. A useful approximation for the momentum transfer at small $\Delta \omega$ is:

$$
q \approx 2 \hbar k_{\omega} \sin \vartheta / 2 .
$$

The interaction process can be understood in two ways. In position space it may be interpreted as Bragg scattering, i.e. stimulated Rayleigh scattering of the atomic de Broglie wave at the optical grating induced by the standing wave and subsequent interference of the phase-modulated de Broglie sidemodes. Alternatively, it can be interpreted in momentum space as Compton scattering, i.e. stimulated Raman scattering between two different motional states of the atoms. The recently observed Recoil-Induced Resonances (RIR) [214] are another manifestation of the same process.

Compton Picture. - In the Compton picture, the atoms being in the standing wave light field may absorb photons from any of the two laser modes and be stimulated to reemit the photons into the modes. Let us assume that an atom with momentum $\mathbf{p}_{i}$ first absorbs a photon of frequency $\omega$ from the laser beam $\mathbf{k}_{\omega}$ and is then stimulated by the laser beam $\mathbf{k}_{\omega+\Delta \omega}$ to emit a photon of frequency $\omega+\Delta \omega$ and to acquire the final momentum $\mathbf{p}_{f}$ (Fig. 18). Because this is a two-photon process, its amplitude is proportional to the square of the light field amplitude, and thus to the light intensity:

$$
H_{\text {Compton }}(\mathbf{r}, t) \sim I(\mathbf{r}, \mathbf{t}) \sum_{\mathbf{p}_{i}}\left|\mathbf{p}_{f}\right\rangle\left\langle\mathbf{p}_{i}\right|+\text { c.c. }
$$

The momentum $\mathbf{q}=\mathbf{p}_{f}-\mathbf{p}_{i}$ and the energy $\hbar \Delta \omega=p_{f}^{2} / 2 m-p_{i}^{2} / 2 m$ are transferred to the atom, so that the atom must follow the Bragg condition:

$$
\hbar \Delta \omega=\frac{q^{2}}{2 m}+\frac{\mathbf{p}_{i} \cdot \mathbf{q}}{m} .
$$

The Bragg condition (5.7) can also be fulfilled by higher-order Raman scattering processes, as we can see by substituting $q \rightarrow n q$, where $2 n$ is the number of absorbed and reemitted photons. This general case is depicted in Figure 18 for $\mathbf{p}_{i}=0$.

Bragg Picture. - In order to explain the scattering process in the Bragg picture, we choose our reference frame so that $\mathbf{k}_{\omega}=-\mathbf{k}_{\omega+\Delta \omega}$ in equation (5.3). In this moving frame, the standing wave amplitude can be written as $E(z, t)=2 E_{0} \cos k_{\omega} z \sin \omega t$. The (single-photon) Rabi frequency $\Omega$ generated by a single travelling wave laser beam has been introduced in equation (3.4). For large red-detuned laser frequencies, $|\Delta| \gg \Omega$, the standing wave creates a light shift modulation described by

$$
U(z)=U_{0} \cos ^{2} k_{\omega} z,
$$


where $U_{0}=\hbar \Omega^{2} / \Delta$ according to equation (3.9). Consequently, the condensate matter-wave develops a spatial phase modulation according to:

$$
\psi(z, t)=\psi_{0}(z) \exp \left[i \hbar^{-1} U(z) t\right]=\psi_{0}(z) \sum_{n} \Im_{n}^{2}\left(U_{0} t / 2 \hbar\right) \exp \left(2 \pi i n k_{\omega} z\right) .
$$

The condensate wavefunction evolves into a superposition of sidemodes, which are just the diffraction orders of the Bragg scattering and whose strengths are given by the Bessel functions $\Im_{n}$. The diffraction efficiency increases with laser intensity and with time.

The above description neglects the atomic motion during the interaction with the standing wave. This thin grating approximation can be satisfied in experiment by irradiating the standing wave only for very short times. The time scale is set by the oscillation period of the atoms in the optical potential valleys generated by the standing wave via the dipole force interaction. At the locations of the antinodes, we may harmonically approximate the potential and introduce the secular frequencies $\omega_{\text {opt }}$ by $\frac{m}{2} \omega_{o p t}^{2}=k_{\omega}^{2} U_{0}$. The thin grating approximation holds for laser pulse durations $\tau \ll 2 \pi / \omega_{\text {opt }}$. For longer pulse durations, in the thick grating limit, the atoms perform on average several oscillations in the optical potential during the interaction time. This causes periodic focussing (decollimation) and defocussing (collimation), which manifests itself in a oscillating Bragg diffraction efficiency.

Bragg diffraction of Bose-condensates was first experimentally observed by Kozuma et al. [20] in the thin grating limit. They briefly irradiated a standing wave into a trapped Bose-condensate, then released the BEC from the trap and recorded the momentum distribution with standard time-of-flight imaging. They observed a splitting of the condensate wavefunction into the Bragg diffracted modes. The efficiency of the Bragg diffraction could be made as high as $100 \%$. By variation of the relative detuning $\Delta \omega$, the diffraction orders could be selected. Subsequent experiments also investigated the thick grating limit [215], by applying the standing wave pulse to released condensates and arranging for large secular frequencies $\omega_{\text {opt }}$.

The BEC Bragg scattering technique described above displays many similarities with acousto-optical modulators (AOMs), which are commonly used in laser optics. However, while AOMs deflect photons passing through the interaction zone, the matter-wave Bragg scattering described here is a time-domain process. This diffraction method constitutes an important atom optical device that will certainly prove a powerful tool in many applications. It has already been used to excite phonons in a controlled way (Section 5.4.2) and to study the intrinsic phase of a condensate [137, 207]. In the reference [207], small condensate replica sequentially generated from a large BEC by coherent Bragg diffraction interfered with each other and yielded information about intrinsic phase variations of the BEC. In reference [216], the Bragg diffraction scheme has been extended to demonstrate a time domain matter-wave analogue of the Talbot effect. And in reference [209], a Bragg diffraction interferometer has been used to map the autocorrelation function of a BEC and to image its phase evolving in time.

\subsection{Nonlinear Atom Optics}

In classical nonlinear optics the interaction between matter (e.g. dilute gases) and light is described by Maxwell's equations:

$$
\begin{aligned}
\mathbf{P}(\mathbf{r}, t) & =\chi(\mathbf{E}) \mathbf{E}(\mathbf{r}, t)=\chi^{(1)} \cdot \mathbf{E}+\chi^{(3)}: \mathbf{E E E}+\ldots \\
\square \mathbf{E}(\mathbf{r}, t) & =\frac{4 \pi}{c} \ddot{\mathbf{P}}(\mathbf{r}, t) .
\end{aligned}
$$

The electromagnetic field $\mathbf{E}$ creates a macroscopic polarization $\mathbf{P}$, which in turn acts back on the field via $\square \mathbf{E}$. Higher order processes like self-focussing, second harmonic generation, four-wave mixing, etc. are described by the nonlinear susceptibility $\chi^{(3)}$. These processes require the presence of a nonlinear medium, because the polarizability of the vacuum itself is pretty small. For visible wavelength the photon-photon scattering crosssection is well approximated by $45^{-2}(973 / 5)\left(\alpha^{4} / \pi\right)\left(\hbar^{8} \omega^{6} / m_{e}^{8} c^{14}\right)$, which is only on the order of $10^{-63} \mathrm{~cm}^{2}$ [217] and very difficult to reach even with high intensity lasers. In contrast to this, the scattering cross-section for shapeless two-body collisions in ultracold sodium gases is on the order of $2 \times 10^{-12} \mathrm{~cm}^{2}$, so that two-body collisions are frequent processes at currently available densities and temperatures. 


\subsubsection{Self-Defocussing}

Two-body collisions play a role in coherent matter-wave optics which is very similar to that of the nonlinear susceptibility in quantum optics. Within the mean-field theory, the groundstate wavefunction of the condensate is described by the nonlinear Schrödinger equation:

$$
\left[\frac{-\hbar^{2}}{2 m} \Delta+U_{\text {trap }}(\mathbf{r})+g|\psi(\mathbf{r}, t)|^{2}\right] \psi(\mathbf{r}, t)=\mu \psi(\mathbf{r}, t)
$$

where $g \equiv 4 \pi \hbar^{2} a / m$. The nonlinear term describes the condensate self-interaction and is analogous to the third order contribution to the polarization in the nonlinear Maxwell equations (5.10). If the atomic interaction is repulsive, the nonlinear term causes the condensate to expand as far as the trapping potential permits. This behavior is analogous to the nonlinear optical self-defocussing in local Kerr media with instantaneous response. For large condensates, the self-interaction can be so overwhelming, that the kinetic energy may be neglected (at least in the center of the trap, where the density is highest). This approximation defines the so-called Thomas-Fermi limit.

\subsubsection{Dispersion}

The nonlinear mean-field interaction in a weakly interacting condensate is at the origin of the phenomenon of dispersion, i.e. the de Broglie wavelength of a single atom with a given momentum $\mathbf{p}$ inside the condensate depends on the local density. For homogeneous condensates, the dispersion relation (2.50) can easily be derived from the semiclassical Bogolubov equations (Section 2.6). In the Thomas-Fermi limit, the region inside the condensate has a nearly homogeneous density, $n(\mathbf{r}) \approx n_{0}$, so that the Bogolubov dispersion describes the excitation spectrum quite well. For low excitation energies, $\varepsilon_{r e c}(\mathbf{p}) \equiv \mathbf{p}^{2} / 2 m \ll g n_{0}$, the spectrum is phononlike (quasi-particle-like):

$$
\varepsilon_{\text {phon }}(\mathbf{p})=c_{s} \mathbf{p} \quad \text { where } \quad c_{s}=\sqrt{g n_{0} / m} .
$$

The excitation energy then depends linearly on the momentum, and density perturbations travel without spreading inside the condensate at the speed $c_{s}$ of the Bogolubov $0^{t h}$ sound. In contrast, for high energy excitations, $\mathbf{p}^{2} / 2 m \gg g n_{0}$, the spectrum is particlelike:

$$
\varepsilon_{\text {part }}(\mathbf{p})=\mathbf{p}^{2} / 2 m+g n_{0}
$$

Phonon-like collective excitations have been driven very soon after the achievement of Bose-Einstein condensation using trap modulation methods (Section 4.3.1). The excitation energies were quite low, i.e. in the same order of magnitude as the trap secular frequencies, $\varepsilon_{\text {phon }}(\mathbf{p}) \approx \hbar \omega_{\text {trap }}$. The de Broglie wavelength of the phonons is then comparable to the condensate size, so that the phonon spectrum is influenced by boundary conditions. It is interesting to tap other regimes of excitation energies that are free from this limitation. The newly developed Bragg diffraction technique can be employed to optically imprint high energy phonons and even particle-like excitations into the condensate 138 and thus to investigate the boundary between these two regimes. Bragg diffraction has been observed earlier with a two laser beam standing wave arrangement as splitting of the matter-wave in momentum space [20] (Section 5.3.3). The energy transfer $\varepsilon_{\text {rec }}(\mathbf{p})$ could be tuned by adjusting the angle between the two laser beams according to equation (5.7) (Fig. 18). We have also seen, that the efficiency of Bragg scattering atoms into the first diffraction order depends on the fulfillment of the Bragg condition (5.7), i.e. Bragg scattering is velocity-selective. One can therefore measure the number of deflected atoms versus the relative detuning of the lasers that drive the Raman transition, record the recoil-induced resonances and call this procedure Bragg spectroscopy [137]. The spectrum closely reflects the momentum distribution of the atoms. Since the mean-field interaction causes a finite momentum spread of the condensate wavefunction, the shift and broadening of the RIR reveal detailed information about the condensate self-interaction (Fig. 18).

For a real condensate, there are several contributions to the width of the momentum distribution: 1) The finite size of the trapped condensate limits the width of the momentum distribution according to Heisenberg's uncertainty relation [44]. 2) The inhomogeneous density distribution of the trapped condensate shifts and smears 
out the momentum distribution in equation (5.13). Since this is an inhomogeneous broadening, it adds to the other linewidth as a quadrature sum. 3) The finite length of the Bragg scattering pulse produces a broadening analogous to the time-of-flight broadening in atomic beam spectroscopy, which is inversely proportional to the pulse length. 4) Acoustic noise may Doppler-broaden the linewidth of the frequency difference of the lasers and reduce the resolving power of the Bragg spectroscopy scheme. The shifts and broadenings of the recoil-induced resonances have been calculated for a realistic condensate density distribution and verified in two experiments, one carried out in the particle regime [137] and one in the phonon regime [138].

Stenger et al. 137] performed the particle regime Bragg scattering experiment, $\varepsilon_{r e c}(\mathbf{p}) \gg g n_{0}$, with counterpropagating laser beams. For this case, the recoil shift for sodium condensates at fulfilled Bragg condition (5.7) was $\varepsilon_{r e c}(\mathbf{p})=h \times 100 \mathrm{kHz}$, which was much larger than the mean-field energy at typical condensate densities, $g n_{0}=g \times 5 \times 10^{14} \mathrm{~cm}^{-3}=h g \times 7.3 \mathrm{kHz}$. The experiment could closely reproduce the expected shift and broadening of the RIR shown in Figure 19 (a).

Stamper-Kurn et al. [138] carried out the phonon regime Bragg scattering experiment with laser beams enclosing an angle of $14^{\circ}$. In this case, the recoil shift at fulfilled Bragg condition (5.7) was $\varepsilon_{r e c}(\mathbf{p})=h \times 1.54 \mathrm{kHz}$, which was now smaller than the mean-field energy at typical densities. The results of this experiment were found in good agreement with calculations of the shift and strength of the RIR shown in the Figures 19 (b) and (c). In order to understand the density dependence of the RIR, we first have a look at the spectrum of light scattered from a homogeneous dilute gas of atoms. If the gas is non-degenerate, the spectrum mirrors the velocity distribution of the atoms. In the presence of condensed atoms, photon recoil events that take atoms to an already occupied state are enhanced by Bose-stimulation, and if the atoms do not interact, according to Javanainen et al. [218, the spectrum $S(\mathbf{p}, \varepsilon)$ exhibits two characteristic peaks at $\varepsilon= \pm \varepsilon_{\text {rec }}(\mathbf{p})$. Later, Graham et al. 219] extended the calculations by taking into account two-body collisions and found the characteristic peaks at an energy $\varepsilon= \pm \varepsilon_{B o g}(\mathbf{p})$ given by the Bogolubov dispersion relation (2.50):

$$
S(\mathbf{p}, \varepsilon)=\frac{\varepsilon}{\varepsilon_{B o g}(\mathbf{p})} \delta\left(\varepsilon_{B o g}(\mathbf{p})-\varepsilon\right) .
$$

The experiments of Stamper-Kurn and Stenger [137, 138] measured exactly these spectra. However, instead of looking at scattered photons, they analyzed the shifts, widths and strengths of the recoil-induced Bragg resonances. They measured, in particular, the line strength, $S(\mathbf{p})=\int S(\mathbf{p}, \varepsilon) d \varepsilon$, and the shift from the free particle resonance, $\Delta \varepsilon \equiv \varepsilon_{B o g}(\mathbf{p})-\varepsilon_{r e c}(\mathbf{p})$, as a function of the mean-field energy. In order to compare with the experiment, the formula (5.14) needs to be slightly modified to take into account the inhomogeneity of the trapped condensate. Figures 19 (b) and (c) show the shift and strength of the RIR as a function of the condensate density. At low densities, when the excitations are particle-like, the line shift tends to zero, $\varepsilon_{\text {Bog }}(\mathbf{p}) \approx \varepsilon_{\text {part }}(\mathbf{p}) \rightarrow \varepsilon_{\text {rec }}(\mathbf{p})$, and the line strength tends to its maximum value, $S(\mathbf{p}) \rightarrow 1$. At high densities, the excitations are phonon-like, $\varepsilon_{B o g}(\mathbf{p}) \approx \varepsilon_{\text {phon }}(\mathbf{p})>\varepsilon_{\text {rec }}(\mathbf{p})$, and the RIR is shifted towards higher energies, while the line strength rapidly decreases. The relative weakness of phonon-like excitations is due to the presence of correlated pair excitations. The direct comparison of the two regimes of excitations thus reveals important information about correlation effects 138.

It is interesting to note, that the spectrum is equivalent to the structure factor, which is itself the Fourier transform of the density correlation function of the condensate quantum field. The structure factor plays a similar role in the theory of many-body Schrödinger fields as the familiar $Q$-function in quantum optics. The correlations are probed by scattering quasiparticles back and forth:

$$
S(\mathbf{p}) \sim\left\langle g\left|\widehat{a}_{\mathbf{p}} \widehat{a}_{\mathbf{p}}^{+}+\widehat{a}_{-\mathbf{p}}^{+} \widehat{a}_{-\mathbf{p}}+\widehat{a}_{-\mathbf{p}}^{+} \widehat{a}_{\mathbf{p}}^{+}+\widehat{a}_{\mathbf{p}} \widehat{a}_{-\mathbf{p}}\right| g\right\rangle,
$$

where $\widehat{a}_{\mathbf{p}}$ stands for the annihilation of a phonon with wavevector $\mathbf{p}$.

\subsubsection{Second Harmonic Generation}

The elementary excitations (i.e. small oscillations around the many-body ground state) discussed in Section 4.3.1 are well described by a linearized Gross-Pitaevskii equation. In contrast, large amplitude oscillations are sensitive to anharmonicities induced by the nonlinear mean-field interaction. Nonlinear effects may result in frequency shifts of the normal modes and mode coupling. 
For mode coupling, the anisotropy of the trapping potential plays an important role. Dalfovo et al. 220] calculated the excitation frequencies for the normal modes of cylindrically symmetric traps $\left(\omega_{r}, \omega_{z}\right)$. The modes are usually labelled with the projection of the angular momentum onto the symmetry axis $m$. The lowest lying modes are the breathing mode (high-lying $m=0$ ), the radial compression oscillation with axial sloshing (low-lying $m=0)$, and the quadrupolar radial shape oscillation $(m=2)$. The oscillations depend differently on variations of the trap geometry. For example, at the aspect ratio $\omega_{z} / \omega_{r}=\frac{1}{6} \sqrt{77+5 \sqrt{145}}$, the excitation frequencies are shifted such that $\omega_{\text {high }}(m=0)=2 \omega_{\text {low }}(m=0)$. Thus, through active control of the trap aspect ratio, it is possible to arrange for degeneracies of the modes where the anharmonic mixing diverges. Under such conditions, frequency doubling effects should occur analogous to Second Harmonic Generation (SHG) in quantum optics.

Second harmonic generation has recently been observed in the collective dynamics of a Bose-Einstein condensate by Hechenblaikner et al. 221]. They modified the potential of their TOP trap by an additional magnetic field oscillating along the symmetry axis with twice the frequency of the rotating bias field. In the time-average, this trap has a variable aspect ratio which can be set by the amplitude of the additional field. Similar to earlier experiments [129, 130], the hydrodynamic mode was excited by sinusoidal modulation of the rotating bias field amplitude. The response of the condensate wavefunction, i.e. the shape oscillation, was observed by standard time-of-flight imaging. When the aspect ratio of the trap was set to the degeneracy condition, the condensate responded nonlinearly by oscillating with twice the driving frequency.

In contrast to light, the material de Broglie wave also depends on the particle's mass. Therefore, modifying the mass and keeping the momentum fixed modifies the de Broglie wavelength. Two free atoms can be coherently coupled to a molecular bound state. The coupling may be realized through a Feshbach resonance 222] (Section 6.1) or by exciting a Raman transition with laser beams 223] (Section 6.3). This process may also be understood as Second Harmonic Generation.

\subsubsection{Four-Wave Mixing and Phase Conjugation}

The idea of phase conjugation with coherent matter-waves has been proposed by Goldstein et al. [224]. The authors proposed dropping a condensate onto a $c w$ standing light wave which was tilted by the Bragg angle from the horizontal plane. When falling through the standing wave, a first-order Bragg diffracted BEC would be generated. This wavepacket would four-wave mix with the zero-order diffracted BEC and the falling BEC to create a phase conjugate BEC. Just recently, Four-Wave Mixing (4WM) has been experimentally demonstrated. Slightly different from the proposal [224, Deng et al. [21] produced three condensates out of one right inside the trap using the method of Bragg scattering described in Section 5.3.3. The scattering process produced the three condensate parts in the same region of space, but with different momenta. The initially overlapping condensates carried out half collisions that nonlinearly mixed the de Broglie waves before they flew apart.

The temporal evolution of four-wave mixing BEC wavepackets has been numerically investigated by Trippenbach et al. [225]. They considered three BEC wavepackets with the initial atomnumbers $N_{j}^{0}$ and wavefunctions, $\psi_{0}\left(\mathbf{r}-\mathbf{r}_{j}\right), j=1,2,3$, each one being the solution of a Gross-Pitaevskii equation (GPE) with a potential centered around $\mathbf{r}_{j}$. The initial locations $\mathbf{r}_{j}$ and the initial momenta $\mathbf{p}_{j}$ were chosen to let the three wavepackets perform full collisions. The evolution of the total wavefunction $\psi_{\text {tot }}(\mathbf{r}, t)$ starting from the initial state $\psi_{t o t}(\mathbf{r}, 0)=\sum_{j=1}^{3} N_{j}^{0} \psi_{0}\left(\mathbf{r}-\mathbf{r}_{j}\right) \exp \left(\frac{i}{\hbar} \mathbf{p}_{j} \cdot \mathbf{r}\right)$ was monitored by solving the time-dependent GPE (2.45). The wavepackets mix due to the nonlinear mean-field interaction term in the GPE giving birth to new wavepackets $\psi_{4} \sim g \psi_{j}^{+} \psi_{m} \psi_{n} \exp \frac{i}{\hbar} \mathbf{p}_{4} \cdot \mathbf{r}$ with momenta $\mathbf{p}_{4}=-\mathbf{p}_{j}+\mathbf{p}_{m}+\mathbf{p}_{n}$. Mixing configurations like $\psi_{j}^{+} \psi_{j} \psi_{j}$ and $\psi_{j}^{+} \psi_{j} \psi_{m}$ do not produce wavepackets with new momenta $\mathbf{p}_{4} \neq \mathbf{p}_{j}, \mathbf{p}_{m}, \mathbf{p}_{n}$, but describe self-phase modulation (Section 5.4.1) and cross-phase modulation, respectively. Only terms that combine atoms from all three wavepackets can produce new momenta. Further restrictions on the possible mixing configurations $j, m, n=1,2,3$ arise from particle number, momentum and energy conservation laws:

$$
\begin{aligned}
N_{4} & =-N_{j}+N_{j}^{0}=N_{m}-N_{m}^{0}=N_{n}-N_{n}^{0}=\sum_{\kappa=1}^{3}\left(N_{\kappa}^{0}-N_{\kappa}\right) \\
\mathbf{p}_{4} & =-\mathbf{p}_{j}+\mathbf{p}_{m}+\mathbf{p}_{n} \\
p_{4}^{2} & =-p_{j}^{2}+p_{m}^{2}+p_{n}^{2} .
\end{aligned}
$$


In order to generate three BEC wavepackets with different momenta, Deng et al. 21 applied two short Bragg diffraction sequences in rapid succession. The geometry of the standing wave laser beams is shown in Figure 20 (a) in the laboratory frame. The first standing wave was generated by lasers $\mathbf{k}_{1}$ and $\mathbf{k}_{2}$ detuned from one another, so that the Bragg condition (5.7) was satisfied and the momentum $\mathbf{p}_{2}=\hbar \mathbf{k}_{1}-\hbar \mathbf{k}_{2}$ was imparted to the diffracted atoms. The second standing wave was formed by the lasers $\mathbf{k}_{1}$ and $\mathbf{k}_{3}=-\mathbf{k}_{1}$ and transferred the momentum $\mathbf{p}_{3}=2 \hbar \mathbf{k}_{1}$ to the atoms. The durations and intensities of the standing waves were adjusted to distribute the atoms in more or less equal parts into the three momentum states $\mathbf{p}_{1}=0, \mathbf{p}_{2}$ and $\mathbf{p}_{3}$. A fourth momentum state $\mathbf{p}_{4}$ was generated by four-wave mixing.

The conservation laws only permit processes that can be viewed as degenerate $4 \mathrm{WM}$ in an appropriate reference frame. Figure 20 (b) shows the process in a moving frame defined by $\mathbf{p}_{1} \equiv-\mathbf{p}_{3}$. Two atoms from $\psi_{1}$ and $\psi_{3}$ are bosonically scattered by an atom from $\psi_{2}$ into the wavepackets $\psi_{2}$ and $\psi_{4}$. Each of the wavepackets $\psi_{1}$ and $\psi_{3}$ sacrifices $N_{4}$ atoms to create the new wavepacket $\psi_{4}$ and to increase the wavepacket $\psi_{2}$. The redistribution is coherent. Figure 20 (c) shows the process in a moving frame defined by $\mathbf{p}_{1} \equiv-\mathbf{p}_{2}$. Energy conservation only allows the terms satisfying $p_{4}=p_{3}$. These terms are $\psi_{1}^{+} \psi_{2} \psi_{3}$ and $\psi_{2}^{+} \psi_{1} \psi_{3}$. In this frame, the process may be interpreted as Bragg scattering of wavepacket $\psi_{3}$ by the matter-wave grating formed by $\psi_{1}$ and $\psi_{2}$. The wavepacket $\psi_{4}$ is just the first-order Bragg diffracted wavepacket. In contrast to Bragg diffraction at an optical grating (Section 5.3.3), Bragg diffraction at a matter-wave grating relies on nonlinear mixing by twobody collisions. The amount of redistributed atoms therefore depends on parameters like the atomic interaction strength, the condensate size, and the collision time, i.e. the time that the wavepackets spend together before they separate. Time-of-flight images of the total condensate wavefunction after 4WM are shown in Figure 21.

The occurrence of four-wave mixing was foreseeable in view of the equivalence between the nonlinear coupling strength $g$ in the Gross-Pitaevskii equation (5.11) and the higher-order susceptibility $\chi^{(3)}$ in nonlinear optics, which is known to produce such phenomena. But despite the similarities with the optical counterpart, fourwave mixing with matter-wave is fundamentally different. Particle numbers must be conserved and the energymomentum dispersion relation is different from the one that holds for massless photons. Furthermore, while photons generally require the presence of a nonlinear medium to undergo higher-order processes, the atomic matter-waves mix via binary collisions.

\subsubsection{Spin Mixing}

In the four-wave mixing scheme discussed above, the nonlinearly interacting condensates are distinguished by their different center-of-mass momenta. Another possibility is to nonlinearly mix overlapping BECs in different internal states, e.g. Zeeman substates. The experimental feasibility of confining spinor condensates of sodium atoms distributed over all $F=1$ hyperfine states in the same trap has triggered extensive theoretical work [28, 128]. Spin-exchange interactions constantly mix the different spin components and drive complex nonlinear spin population dynamics. For example, two $m_{F}=0$ atoms may collide and change their internal state to one $m_{F}=-1$ and one $m_{F}=+1$ atom. A recent experiment has demonstrated, how a condensate (initially in the $m_{F}=0$ state) evolves into a mixture of populations of all three hyperfine states and subsequently forms spin domains [8]. However, the observation of nonlinear spin mixing is a challenge still lying ahead.

\subsubsection{Dielectric Properties of Bose-Einstein Condensates}

In the preceding sections, we discussed several matter-wave effects with Bose-Einstein condensates that were due to their intrinsic collision-induced nonlinearity. However, regardless of this atom optical nonlinearity, Bose-condensed gases can also behave as highly dielectric media for light and be useful objects for studies in nonlinear quantum optics.

Under normal circumstances, the refractive index of a gas can only be increased at the detriment of transmission. However, in a gas of laser-driven $\Lambda$-shaped atomic three-level systems, quantum interference can occur cancelling out the absorption and leaving transparent the otherwise opaque medium. The phenomenon is termed Electromagnetically Induced Transparency (EIT). In this system, when both lasers are tuned to resonance, the excited state is not populated and can be adiabatically eliminated. Upon tuning one of the lasers, a dark resonance can be observed whose width is power-broadened by the laser intensities, if Doppler broadening, broadening by 
laser phase fluctuations or by collisions between atoms can be neglected [226]. Close to the dark resonance, the dispersion (i.e. the frequency dependence of the refractive index) is very large. It depends on the width of the dark resonance. The group velocity for a propagating light pulse is $v_{g}=c\left(n\left(\omega_{\text {probe }}\right)+\omega_{\text {probe }} \frac{d n}{d \omega_{\text {probe }}}\right)^{-1}$, where $n\left(\omega_{\text {probe }}\right)$ is the refractive index at the probe beam frequency $\omega_{\text {probe }}$. The propagation velocity is slowed down if the dispersion is large 227.

Taking advantage of their sodium BEC apparatus, Hau et al. [228] produced a dense $\left(n \sim 8 \times 10^{13} \mathrm{~cm}^{-3}\right)$ gas of ultracold $(T \sim 400 \mathrm{nK})$ atoms in an oblong magnetic trap and probed the cloud in-situ and time-resolved. They shone along the long axis of the cloud a short pulse of circularly polarized probe light resonantly tuned between the levels $\left|F^{\prime}=2, m_{F}^{\prime}=-2\right\rangle$ and $\left|F=1, m_{F}=-1\right\rangle$. The transmitted pulse is detected with a photomultiplier. In the presence of a low-intensity linearly polarized laser beam irradiated perpendicular to the probe beam and tuned between the levels $\left|F^{\prime}=2, m_{F}^{\prime}=-2\right\rangle$ and $\left|F=2, m_{F}=-2\right\rangle$ which dressed the atomic cloud and kept the probe light from being absorbed, the light pulse was delayed. If BECs were used, the delay corresponded to a speed of light on the order of only $17 \mathrm{~m} / \mathrm{s}$. This corresponds to an unprecedentedly large nonlinear refractive index. Inouye et al. 2229] later reported light group velocities of $1 \mathrm{~m} / \mathrm{s}$ in the context of their experiment on the amplification of light and atoms in a BEC (Section 5.5.2). It is worth mentioning, that the effect does not require quantum degeneracy, but rather high density and low temperature, and a similar reduction of the speed of light has subsequently been observed in hot gases [230]. The group velocity reduction scales with the gas density and inversely with probe beam intensity. At low temperature, one can afford lower probe beam intensity without being dominated by the Doppler effect.

Such strong nonlinearities may prove useful for a variety of applications in nonlinear quantum optics. An interesting proposal [231] points out, that strongly dielectric moving media may exhibit detectable relativistic effects of light when the speed of light gets comparable to the local speed of sound or the flow of mass. In particular, a vortex flow imprints a long-ranging topological phase shift on incident light that can be understood in terms of an optical Aharonov-Bohm effect. This may prove useful for the detection of quantum vortices in BECs (Section 4.3.4). At short ranges, vortices should behave similar to gravitational black holes and deviate light towards the vortex singularity. Beyond an "optical Schwarzschild radius", the light is trapped by the vortex.

\subsection{Coherent Coupling of Optical Fields and Matter-Waves}

\subsubsection{Superradiant Rayleigh Scattering}

An early example for the influence of the dynamic coupling between optical fields and matter-waves on the centerof-mass motion of the material system is the Free Electron Laser (FEL). In this device, a combination of periodic magnetic and optical fields causes a spatial density modulation of a relativistic electron beam. This modulation generates an oscillating current which amplifies the optical field and increases the density modulation again, thus initiating a runaway amplification process. In an appropriate reference frame, the fundamental mechanism that coherently scatters photons into the optical field can be understood as cooperative Compton scattering or Bragg scattering of the particles (i.e. electrons) at a moving standing light wave. This point of view together with the experimental observation of recoil-induced resonances in atomic gases [214] triggered a few years ago the idea of an atomic analogue to the FEL: the Collective Atomic Recoil Laser (CARL) [232]. In the CARL, photons are coherently redistributed between the modes of a moving standing light wave by mediation of the atomic center-of-mass motion. Cooperative Compton scattering leads to collective atomic recoil and self-bunching of the matter-wave which results in exponential gain. The recent observation of Bragg scattering in Bose-condensates brought up the question whether BECs could serve the purpose of an ultra-cold version of the CARL [29]. The superradiant Rayleigh scattering of laser light by a BEC seen by Inouye et al. 222] already shows several features peculiar to CARL. The long coherence time of BECs strongly correlates successive Rayleigh scattering events via long-lived quasiparticle excitations. The positive feedback of these excitations on the laser light results in exponential gain and directional bundling of the scattered light.

When an incoming photon with wave vector $\mathbf{k}_{0}$ is scattered by a condensed atom into the mode $\mathbf{k}_{s}$, with $k_{s}=k_{0}$, this atom receives the recoil momentum $\mathbf{q}=\hbar \mathbf{k}_{s}-\hbar \mathbf{k}_{0}$ and, while it propagates with a speed of a few 
centimeters per second through the condensate, it interferes with the other atoms of the BEC to form a matterwave grating. The grating, which is long-lived compared to the scattering rate, now stimulates subsequent photons from the incoming laser beam to scatter into the same direction $\mathbf{k}_{s}$ and for its part picks up the recoiled atoms. The process is self-amplifying, i.e. the number of photons in $\mathbf{k}_{s}$ grows exponentially in time. The scheme can also be interpreted the other way round as scattering of atoms into the BEC momentum sidemode $\mathbf{q}$ stimulated by spontaneously scattered photons and bosonically enhanced by the numbers of atoms already being in the sidemode. The inversion that produces the exponential gain is readily understood in a dressed atom picture. The resting BEC and the irradiated laser light form together an excited state that decays into recoiling atoms and scattered photons. The photons quickly leave the BEC which maintains the inversion and permits, in principle, the complete transfer of the BEC into the momentum sidemode. The spontaneity of the scattering process ensures the irreversibility of the gain process.

For their experiment, Inouye et al. produced a cigar-shaped sodium condensate with spatial extensions $z_{r m s}=200 \mu \mathrm{m}$ and $r_{r m s}=20 \mu \mathrm{m}$ confined in a cloverleaf trap. The BEC was irradiated from a radial direction with a single linearly polarized laser light pulse tuned $\Delta=-1.7 \mathrm{GHz}$ below the $D 2$ line. The variable laser intensity, $I=1 . .100 \mathrm{~mW} / \mathrm{cm}$, and duration, $\tau=10 . .800 \mu \mathrm{s}$, permitted the adjustment of the single-atom far-off resonance Rayleigh scattering rate to $R \approx I / \hbar \omega \times \sigma_{0} \Omega^{2} / 4 \Delta^{2}=45 . .4500 \mathrm{~s}^{-1}$. After the application of the laser pulse, the magnetic trap was switched off and a time-of-flight picture was taken after $20 . .50 \mathrm{~ms}$ of free expansion (Fig. 22). Additionally, the scattered light could be recorded either spatially resolved with a CCD camera or time-resolved with a photomultiplier. In the following, we will discuss some of the observations made in this experiment.

Rayleigh Scattering. - The total gain depends on the size of the condensate, i.e. the distance over which single-path gain can happen. Mode competition quenches the scattering in all but the maximum gain directions [233]. Non-spherical BECs therefore yield highly anisotropic Rayleigh scattering. The competing process of Raman scattering into different Zeeman sublevels is not bosonically stimulated. For cigar-shaped BECs the gain path is longest along the symmetry axis, which results in so-called end-fire modes. Scattering recoils the atoms and has to stop when all the atoms are transferred to higher momentum sidemodes. Consequently, Inouye et al. observed highly directional fluorescence light bursts along the symmetry axis, whose durations were shortened as the irradiated laser intensity was increased.

Since the end-fire modes enclose a $90^{\circ}$ angle with the incoming laser beam and the frequency of the light does not change during Rayleigh scattering, the scattered matter-wave gets a $45^{\circ}$ momentum kick. The time-offlight images in Figure 22 show the momentum distribution of the condensate after irradiation of a single laser pulse with various durations. For longer pulse durations, repeated Rayleigh scattering at the higher momentum sidemodes gives rise to additional peaks.

Superradiance. - The process is equivalent to Dicke superradiance, where the overlapping radiation fields of a dense sample of excited atomic dipoles stimulate each other to synchronously emit light, thus leaving the sample in a coherent superposition state. The total emission time is reduced to short fluorescence bursts. While in classical superradiance the sample of two-level systems evolves into an oscillating coherence of internal electronic states, in the MIT experiment, we have a coherent oscillation of translational sidemodes. Superradiance does not require quantum degeneracy, but the dipoles must have a long coherence time. Doppler broadening accelerates relaxation. In the MIT experiment, where the coherence is stored in the motional degrees of freedom, having long coherence time is equivalent to having a large coherence length. BECs have a large coherence length that corresponds to their size, while for thermal clouds the coherence length is just its thermal de Broglie wavelength. This explains why Inouye et al. could not observe superradiance using thermal clouds.

The superradiance was found to be very sensitive to the polarization of the incoming laser light. Since the atoms were polarized in axial direction by the magnetic field of the cloverleaf trap, photons polarized in the same direction were absorbed and spontaneously reemitted according to the (torus-shaped) dipole radiation pattern for $\pi$ radiation, i.e. not in axial direction. On the other hand, if the laser beam was polarized perpendicular to the long $\mathrm{BEC}$ axis, the (bow-tie-shaped) dipole radiation pattern for $\sigma^{ \pm}$radiation supported superradiance. 


\subsubsection{Matter-Wave and Light Amplification}

The superradiance experiment of Inouye et al. realizes a matter-wave amplifier along the lines described by Law et al. 2234] and Moore et al. 235]. The momentum sidemodes which they observed may be regarded as amplified vacuum fluctuations. However, the proof that the amplification process is coherent, i.e. that the original matter-wave has a well-defined phase relation to the amplified matter-wave was still lacking. This proof has recently been provided by two experiments by Kozuma et al. [23] and at the MIT [24, 229].

In extension of the superradiance experiment, the MIT group seeded the matter-wave amplifier with a very small condensate $(\sim 0.1 \%$ of the total condensate) thus substituting the quantum fluctuations in their role of input wavepacket. The seed condensate was provided by a matter-wave Bragg diffraction pulse (Section 5.3.3). It interfered with the main condensate to form a matter-wave grating which was then amplified by a subsequent Rayleigh scattering pulse. The gain in atom number for the seed mode could be set between 10 and 100 by controlling the intensity and duration of the Rayleigh pulse. Inouye et al. also demonstrated the coherence of the amplification process by setting up a Ramsey type active atom interferometer scheme whose one arm consisted of the amplified seed condensate and the other arm of a reference condensate created from the original condensate by Bragg diffraction. The observation of interference proved the coherence of the amplification process.

Kozuma et al. chose a similar approach. They produced an elongated rubidium condensate in a cloverleaf trap and, in contrast to the MIT group, irradiated the superradiance and Bragg diffraction pulses into the long axis of the condensate after releasing it from the trap. They reduced the superradiant gain of their system so much that spontaneous quantum fluctuations were not amplified, produced a seed condensate wavepacket by Bragg diffraction $(\sim 6.5 \%)$ and showed that this was amplified to up to $66 \%$ of the total BEC by a Rayleigh scattering pulse. They could also demonstrate interference between the amplified and the original BEC wavepackets in a Mach-Zehnder type atom interferometric setup [207]. In a traditional Mach-Zehnder atom interferometer, a wavepacket is first split with a $\pi / 2$ interaction pulse, thus recoiling half of the atoms and leaving the other half unaffected. A subsequent $\pi$ pulse reverses the momentum, so that the wavepackets move towards each other. A final $\pi / 2$ pulse recombines the components and produces interference, provided every interaction was really coherent. Kozuma et al. used Bragg diffraction interaction pulses in their Mach-Zehnder interferometer with an essential modification: The first $\pi / 2$ pulse consisted of a combination of a Bragg pulse which produced the seed condensate and a superradiance pulse which amplified it to a size corresponding to half the BEC. The observation of interference thus proved that the first composite $\pi / 2$ pulse maintained the coherence, that the long-range order was preserved for the amplified BEC and that it was phase-locked to the seed BEC. An important drawback for matter-wave amplifiers and atom lasers (Section 5.2.3) is the limited reservoir of atoms. The amplification imperatively stops when all the atoms of the BEC have been transferred into the amplified momentum sidemode.

The atom optical devices listed in Section 5.1.1 are all passive devices. In contrast, the phase-coherent matter-wave amplifiers discussed above actively stimulate the atoms to scatter into the amplified mode. It is worth pointing out the analogy between this scattering process and four-wave mixing. While matter-wave 4WM, which may be viewed as bosonically enhanced redistribution of atoms between momentum sidemodes mediated by the mean-field, involves four atoms (two in the input and two in the output channel) and quantum optical 4WM, which may be viewed as coherent redistribution of photons between light modes, involves four photons, the process underlying the superradiant Rayleigh scattering takes place between two atoms and two photons. In all three cases, bosonic stimulation plays a key role.

We have seen in the superradiance experiment, that the Rayleigh scattered light is stimulated into the endfire modes. The process is self-amplifying and can be used as a light amplifier for optical seed pulses. In a subsequent experiment, Inouye et al. 229 demonstrated the amplification of light pulses. The occurrence of Rabi oscillations in the temporal behavior of the gain showed that the gain process was coherent. 


\subsubsection{Quantum Optics with Atoms}

The intrinsic coherence of Schrödinger fields implies the possibility of "exotic" quantum correlations. Laser light is, normally, best described by a coherent or Glauber state. But other quantum states of light are possible, i.e. squeezed states, Schrödinger cat states, states with sub-Poissonian photon distribution, e.g. pure number or Fock states, and even single photon states. All these states have been observed in ultrahigh finesse micromasers. A mathematically very similar system is the Hilbert space of the motion of a single particle in a harmonic trap, e.g. an ion stored in a Paul trap 175. Non-coherent motional quantum states have been observed by Wineland et al. 176. Quantum correlations have also been studied theoretically in atomic Bose-Einstein condensates, and there are propositions on how to create non-coherent states of BECs [27, 236, 237. (Note that non-coherent state BECs are not less coherent, but contain more complicated quantum correlations than Glauber state field distributions.) This field of investigations may be called "quantum atom optics" in analogy to the field of quantum optics dealing with the non-classical features of light.

At the interface between the macroscopic world and the microscopic quantum world, Schrödinger cat states are epitomized by new theories on quantum decoherence. Schrödinger cat states are coherent superpositions of multi-particle quantum states. A perfect cat state can be written as $|N, 0\rangle \pm|0, N\rangle$, i.e. all particles are in a superposition of two states of an arbitrary degree of freedom, e.g. coordinate, momentum or internal excitation. Because of their large scale, mesoscopic coherent quantum objects like Bose-condensates are ideal testing grounds for studies of fundamental questions on quantum entanglement, quantum measurement, and decoherence. Unfortunately, big Schrödinger cats are extremely sensitive to decoherence. Proposals to generate such states in BECs [27, 236, 237] have been reexamined by Dalvit et al. [238] who also suggested several measures to master the decoherence problem. The decoherence rate $\gamma_{d e c}$ generally depends on the "macroscopicity" $N$ of the quantum state and its contact with the environment. Thermal collisions (occurring at a rate $\gamma_{\text {coll }}$ ) are the main reason for decoherence in a BEC cat, $\gamma_{d e c} \sim N^{2} \gamma_{\text {coll }}$ [238], but Rayleigh scattering and three-body recombination also contribute. In the extreme case of a perfect cat state, the coherence is destroyed by scattering of a single atom, since its detection tells the state of all atoms. It is worth emphasizing that Schrödinger cat states should not be confused with the beamsplitter states discussed in Section 5.2.3, where every single atom has the option of being in one of two states, $(|1,0\rangle \pm|0,1\rangle)^{N}$. Beamsplitter states only involve single-particle correlations and are readily produced by Bragg scattering techniques.

The perfect cat state exhibits maximum entanglement and is, in this respect, similar to Einstein-PodolskiRosen (EPR) and Greenberger-Horne-Zeilinger (GHZ) states. Such states of several entangled particles are currently investigated in the context of quantum computation and have recently been realized with single ions [239] and with micromasers [240]. Controlled collisions in optical lattices may offer new opportunities for entangling neutral atoms and implementing schemes for coherent quantum operations [241. However, while for computational purposes it is necessary to show up with a scheme where the fundamental registers (called qubits) can be individually addressed, the delocalized Bose-condensed atoms do not lend themselves to individual manipulations. Still it is conceivable that new ideas that make use of the mesoscopic coherence of BECs will emerge from the paradigm of coherent entanglement and quantum control between BECs and laser modes.

The theory describing the coherent coupling of optical quantum fields and Bose-Einstein condensates encompasses the classical domains of quantum optics and atom optics as limiting cases. This theory is in many aspects similar to optical cavity-QED theories, and the analogy seeds new ideas about cavity atom optics, entanglement between atomic and laser beams, and optical control of BECs [28]. As an example: in quantum optics the Optical Parametric Amplifier (OPA) generates correlated photon-photon states. Similarly, as we have seen in the superradiant Rayleigh scattering experiments, the coherent interaction between light and BECs creates entangled atom-photon states [233]. The range of possible applications is wide and may include tests of Bell's inequality, quantum cryptography and quantum teleportation. 


\section{Chapter 6}

\section{Collision Resonances}

The mean-field interaction of ultracold dilute atomic gases is dominated by binary $s$-wave collisions. In the shapeless approximation, the collisions can be modelled by a single atomic constant, the scattering length, which measures the low energy phase shift of the relative de Broglie wave of the atoms during a collision process. The scattering length determines the magnitude of the elastic and inelastic collision rates.

However, the scattering length may be manipulated with optical [242] or microwave [243] radiation fields or, close to Feshbach collision resonances, with external magnetic fields 244. Feshbach resonances were first predicted for nuclear systems [245, and have recently regained much attention in the context of Bose-Einstein condensation. They permitted the condensation of a new atomic species (Section 6.2) and are currently investigated in the context of free-bound coupling and the creation of molecular BECs (Section 6.3).

\subsection{Feshbach Resonances in ${ }^{85} \mathrm{Rb}$ and ${ }^{23} \mathrm{Na}$}

Feshbach resonances are collision resonances that occur when the energy of a colliding channel coincides with the energy of a vibrational bound state of a potential that correlates with a higher lying asymptote (Fig. 23). If the bound state and the free atoms have different magnetic momenta, the resonance condition may be tuned via external magnetic fields exploiting the Zeeman-effect. When a Feshbach resonance is crossed, the scattering length goes through a singularity (Fig. 24).

The complex spin-structure of the alkalis results from a combination of exchange, hyperfine and Zeeman interaction and gives rise to a multitude of energy levels, with a good chance of having one or more Feshbach resonances. Verhaar and coworkers [246, 247] performed coupled multi-channel calculations and found Feshbach resonances at experimentally accessible field strengths in ${ }^{85} \mathrm{Rb}$ and ${ }^{23} \mathrm{Na}$.

There are several ways to detect Feshbach resonances. The first way is using Photoassociation Spectroscopy (PA). PA is a frequently used tool to explore the level structure of excited molecular states by irradiating a laser tuned between the colliding channel and a vibrational bound level of the excited state potential [248]. Close to a Feshbach resonance the colliding wavefunction amplitude is enhanced and therefore its Franck-Condon overlap with the excited state wavefunction, and the photoassociative transition rate gets larger. Since the excited molecular state preferentially decays into the dissociation continuum, where the atoms have high kinetic energy, the transition rate may be monitored via trap losses. This method has been employed to detect a broad Feshbach resonance in ${ }^{85} \mathrm{Rb}$ near $B=160 \mathrm{G}$ with $6 \mathrm{G}$ width 12 .

A second method is based on the fact that the elastic cross-section and therefore the collision rate in an atomic gas are both proportional to the square of the scattering length in the limit of very low temperatures. This yields a simple recipe for probing Feshbach resonances: One drives a dense cold cloud out of thermal equilibrium and simply measures the rethermalization speed. It takes on average three collisions per atoms to rethermalize a sample. Close to the Feshbach resonance the rethermalization speed should be drastically enhanced. This method has provided an improved measurement of the location of the ${ }^{85} \mathrm{Rb}$ Feshbach resonance [249], which in turn has been utilized to calibrate the calculations of the ${ }^{85} \mathrm{Rb}$ potentials and to enhance their 
precision to a large extent. On the other hand, the influence of the Feshbach resonance on the collision rate suggests its use to control and improve evaporative cooling of atomic clouds.

A third method is based on the influence of the scattering length on the mean-field energy of Bose-Einstein condensates. In fact, the scattering length is the only atomic parameter showing up in the Gross-Pitaevskii equation. It governs the shape and size of the BEC wavefunction, the BEC dynamics and of course all nonlinear interactions. The effect of a Feshbach resonance on BECs has been studied in ${ }^{23} \mathrm{Na}$ [11]. Unfortunately, this experiment also showed the occurrence of inelastic collision processes close to the Feshbach resonance leading to a strong depletion of the condensate. This will most likely limit the practical use of this resonance in sodium.

\subsection{Bose-Einstein Condensation in ${ }^{85} \mathrm{Rb}$}

The zero-field scattering length of the ${ }^{85} \mathrm{Rb}$ isotope in the ground-state $F=2, m_{F}=-2$ is $a_{|2,-2\rangle} \approx-400 a_{B}$ [12]. The negative scattering length inhibits the formation of stable Bose-Einstein condensates with this atomic species. However, in proximity to a Feshbach resonance the scattering length is very sensitive to ambient magnetic fields, $B$ (Fig. 24), which can alter its value and even its sign.

The JILA group, led by Wieman, recently reached the quantum degenerate regime with ${ }^{85} \mathrm{Rb} 250$ ] operating in a regime of positive scattering length. Efficient evaporation is hindered by a deep notch in the elastic scattering cross section at collision energies around $350 \mu \mathrm{K}$, a peculiarity of ${ }^{85} \mathrm{Rb}$, and by inelastic two- and three-body collisions being very frequent at some regimes of the scattering length. Cornish et al. 250 avoided these difficulties using a relatively weak trap, $\bar{\omega} \approx 2 \pi \times 13 \mathrm{~Hz}$, to reduce the atomic cloud density and by following a sophisticated evaporation path. The low density slowed down the evaporation and required a long magnetic trap lifetime. The first evaporation step was performed in the high-field wing, at $B=250 \mathrm{G}$, far from the Feshbach resonance. When the sample was cooled to $2 \mu \mathrm{K}$, the sign of the scattering length was reversed and its absolute value reduced, $a_{|2,-2\rangle}(B)=290 a_{B}$, by moving the magnetic field strength towards the Feshbach resonance to $B=162.3 \mathrm{G}$ (Fig. 24). This further reduced the inelastic collision rate. The evaporation was now pursued until the condensation threshold was approached with $10^{6}$ atoms. While the condensate formed, inelastic loss processes rapidly reduced the trapped atom number to $10^{4}$ at typically $15 \mathrm{nK}$ temperature and $10^{12} \mathrm{~cm}^{-3}$ density. The BEC lifetime was about $10 \mathrm{~s}$.

Size and shape of the condensate depend on the self-energy and thus on the scattering length. Tuning the magnetic field across the Feshbach resonance changes the condensate shape between the limits of an ideal gas Gaussian density distribution and a Thomas-Fermi regime parabolic distribution. Cornish et al. recorded pictures of the condensate with $1.6 \mathrm{~ms}$ absorptive time-of-flight imaging, determined the scattering length from the shape of the BEC, and confirmed the magnetic field dependence shown in Figure 24. At the magnetic field strength $B=156.6 \mathrm{G}$, the scattering length climbs to $a_{|2,-2\rangle}(B)>10000 a_{B}$, and the measured condensate peak density yielded $n a^{3} \approx 0.01$. In this regime, the dilute-gas assumption $n a^{3} \ll 1$ begins to break down, and effects beyond the mean-field approximation, like characteristic shifts in the frequencies of collective excitations, may be observed.

When the self-energy was reduced by tuning the scattering length from a positive regime away from the Feshbach resonance into a regime where the scattering length is negative (Fig. 24), i.e. beyond $B=166.8 \mathrm{G}$, the BEC exhibited an abrupt dynamical behavior. The BEC shrank until it collapsed ejecting a burst of hot atoms. If we compare to Hulet's experiment (Section 3.2.1) which relies on the ensemble analysis of collapsed condensates, we find that the ability to control the value and the onset of the $a<0$ instability greatly facilitates studies of the collapse dynamics. Also, we expect that the successful realization of BEC in ${ }^{85} \mathrm{Rb}$ taps a whole new field of possibly very interesting investigations with the scattering length as an additional, dynamically tunable degree of freedom.

\subsection{Molecular Bose-Einstein Condensates}

Recent theoretical investigations [26, 222], 251]- 253] have shown, that the physics of Feshbach resonances is considerably richer than that of an altered effective scattering length. Feshbach resonances provide a free-bound coupling between the two-colliding-atoms continuum state and a quasibound vibrational molecular state that 
has some analogy to Second Harmonic Generation (SHG). When the Feshbach resonance is excited in a Bosecondensate, the quasi-molecules are predicted to form a molecular BEC. The atomic and the molecular BEC are coupled via intercondensate tunneling of atom pairs. The system may even exhibit Josephson oscillations as a signature of this novel type of quantum tunneling. Free-bound coupling can alternatively be established by driving two-photon Raman photoassociation transitions [223, 254]. This system closely resembles the Feshbach resonance system and may generate molecular BECs and Josephson oscillations between atomic and molecular BECs as well.

The possibility of using incoherent $P A$ to convert large amounts of free atoms to low-lying vibrational levels of ultracold groundstate molecules has been pointed out by Band et al. 255. Ultracold molecules have recently been produced in such photoassociation schemes [256]. On the other hand, the equilibrium yield of coherent Raman PA depends on the entropies of the coupled systems 223. In a thermal atomic gas, the (quasi-)continuum of dissociated atomic states (they are still confined in a magnetic trap) has a much larger entropy than the discrete spectrum of vibrational molecular states. Therefore, the balance of the coherent free-bound coupling has to be on the side of the continuum, i.e. molecules dissociate more frequently than they associate. Quantitative estimates of the PA rates have to thermally average over transition rates (as opposed to transition amplitudes). As a consequence, coherent processes, even STImulated Raman Adiabatic Passage (STIRAP) transitions, have negligible molecular yield. (In short, STIRAP consists of a counterintuitive pulse sequence of the two frequencies involved in the Raman process.) However, as the atomic cloud approaches quantum degeneracy, the dimensionality of the phase space is reduced to a large extent (a BEC has zero entropy) and the coherent free-bound coupling should transform a considerable amount of condensed atoms into a molecular BEC. In specific schemes, e.g. by quickly removing the molecules from the interaction region [254] or by using two-photon photoassociative STIRAP pulses [257], it should be possible to produce molecules in a controlled manner with unity yield. The formation of molecules within a condensate can be understood as a Bose-stimulated chemical process. The dynamics of this process is driven by quantum statistics rather than by chemical forces between individual atoms.

A possible system to study such phenomena is ${ }^{87} \mathrm{Rb}$ (Fig. 25$)$. Two-photon transitions to very weakly bound vibrational molecular Rydberg states have been observed in a dark MOT [258] and later even in BECs [259]. A narrow linewidth of down to $1.5 \mathrm{kHz}$ of the Raman dark resonance, only observed with quantum degenerate gases, is a clear indication of coherent coupling. The narrow dark resonance permitted the measurement of the binding energy of the molecular state, $E=2 \pi \hbar \times 636.0094 \mathrm{MHz}$, with an unprecedented resolution only limited by the inhomogeneous spatial distribution of the atoms and their self-energy. The molecules were produced at rest, because the Raman process does not transfer momentum. Molecular condensates may form in the groundstate of the trap confining the condensate, provided the dark resonance width is inferior to the trap secular frequencies. Unfortunately, fast inelastic decay into lower vibrational states limits the lifetime of the molecular Rydberg states to less than $1 \mathrm{~ms}$. 


\section{Chapter 7}

\section{Criteria of Bose-Einstein Condensation}

The phenomenon of Bose-Einstein condensation involves several rather delicate concepts, such as coherence and gauge-symmetry breaking. In literature, these concepts are very often misinterpreted. Therefore we feel it is necessary to give an accurate and detailed description of the principal notions lying in the foundation of the considered phenomenon.

One generally implies that the Bose-Einstein condensation is a macroscopic occupation of a single quantum state, usually of the ground state, as it was suggested by Bose [1] and Einstein [2] who considered this phenomenon for ideal gases. For noninteracting atoms, the meaning of single-particle quantum states is well defined. This, however, is not always the case for interacting atoms. In order to formulate more precisely what the Bose-Einstein condensation actually is, several criteria are employed. Here we give a careful analysis of these criteria, of their mutual interrelations, and of their relation to the original idea [1, 20 a macroscopic occupation of the ground state.

Intuitively, one expects that the condensation in a system of $N$ bosons occupying volume $V$ can occur when the thermal wavelength $\lambda_{T}$ becomes much larger than the mean interatomic distance $a$, that is,

$$
\frac{a}{\lambda_{T}} \ll 1, \quad \lambda_{T} \equiv\left(\frac{2 \pi \hbar^{2}}{m_{0} k_{B} T}\right)^{1 / 2}
$$

where $m_{0}$ is the atom mass; $T$, temperature. At the same time, the characteristic interaction radius, $r_{\text {int }}$, has to be much smaller than the mean interparticle distance

$$
\frac{r_{i n t}}{a} \ll 1, \quad r_{i n t} \sim\left|a_{s}\right|,
$$

the interaction radius being of the order of scattering length $a_{s}$. In the other case, strong interaction between atoms could deplete the condensate or even completely destroy it. For the density of particles

$$
\rho \equiv \frac{N}{V} \sim a^{-3},
$$

the above conditions can be written as

$$
\rho \lambda_{T}^{3} \gg 1, \quad \rho r_{i n t}^{3} \ll 1 .
$$

Inequalities (7.1) and (7.2), or (7.3), are the expected conditions for the occurrence of the Bose-Einstein condensation. The discussion of sufficient conditions is given in the following subsections.

\subsection{Einstein Criterion of Condensation}

The statement of a macroscopic occupation of a quantum state [2] can be formalized as follows. Let an orthonormalized basis $\left\{\varphi_{n}(\mathbf{r})\right\}$ be given composed of wave functions corresponding to single-particle quantum 
states labelled by a multi-index $n$. Field operators can be expanded in this single-particle basis as

$$
\psi(\mathbf{r})=\sum_{n} a_{n} \varphi_{n}(\mathbf{r})
$$

with the coefficients

$$
a_{n}=\left(\varphi_{n}, \psi\right) \equiv \int \varphi_{n}^{*}(\mathbf{r}) \psi(\mathbf{r}) d \mathbf{r} .
$$

A physical quantity is called macroscopic if it is proportional to the average number of particles

$$
N \equiv<\hat{N}>=\sum_{n}<a_{n}^{\dagger} a_{n}>
$$

being the statistical average of the number-of-particles operator

$$
\hat{N} \equiv \int \psi^{\dagger}(\mathbf{r}) \psi(\mathbf{r}) d \mathbf{r} .
$$

The quantity $<a_{n}^{\dagger} a_{n}>$ is the occupation number of a quantum state $n$. The occupation is termed macroscopic if $\left\langle a_{n}^{\dagger} a_{n}>\right.$ is proportional to $N$. In equilibrium systems, it is the single-particle ground state, i.e. the state of the minimal single-particle energy, that can become macroscopically occupied, which means that the number of particles in the ground state,

$$
N_{0} \equiv<a_{0}^{\dagger} a_{0}>
$$

can become proportional to $N$. This can be stated more rigorously by means of the limit

$$
\lim _{N \rightarrow \infty} \frac{N_{0}}{N}>0 \text {. }
$$

Condition (7.7) defines precisely what one actually implies when talking about the macroscopic occupation of a quantum state.

However, the criterion of condensation (7.7) has several weak points. First of all, there is an ambiguity in choosing a single-particle basis $\left\{\varphi_{n}(\mathbf{r})\right\}$ which all the following consideration is based on. Such a basis naturally arises and is well defined for ideal gases [260], while for interacting particles it is, in general, not uniquely defined. Hence the single-particle ground state and the related occupation number are not well defined for a system of interacting atoms.

Some delicate problems may appear in defining the limit (7.7), as was shown for an exactly solvable model (Michoel and Verbeure [261). This means the following. The number of particles in the ground state (7.6) can be defined as

$$
N_{0}=\lim _{\delta \rightarrow 0} N_{\delta}, \quad N_{\delta} \equiv \sum_{n=0}^{\delta}<a_{n}^{\dagger} a_{n}>.
$$

It happens sometimes that

$$
\lim _{N \rightarrow \infty} \lim _{\delta \rightarrow 0} N_{\delta}=0
$$

although

$$
\lim _{\delta \rightarrow 0} \lim _{N \rightarrow \infty} N_{\delta}>0 .
$$

It is also worth emphasizing that, when considering the criterion (7.7), one usually tacitly assumes that the macroscopic occupation occurs solely for one quantum level, i.e. for the ground state level. But, in general, the situation may happen when several quantum states, or even an infinite number of them, become macroscopically occupied so that

$$
\lim _{N \rightarrow \infty} \frac{1}{N}<a_{n}^{\dagger} a_{n}>=\text { const }>0
$$

for several quantum numbers $n$. 


\subsection{Penrose Criterion of Condensation}

Penrose 262 and Penrose and Onsager 263 criticized the criterion (7.7) stressing that "this criterion has meaning for noninteracting particles only, because single-particle energy levels are not defined for interacting particles". They suggested a generalization of this criterion valid for interacting particles as well. The generalization is based on the definition of the eigenvalues of the first-order density matrix

$$
\rho_{1}\left(\mathbf{r}, \mathbf{r}^{\prime}\right) \equiv<\psi^{\dagger}\left(\mathbf{r}^{\prime}\right) \psi(\mathbf{r})>
$$

The eigenvalues of the matrix (7.11) are given by the eigenproblem

$$
\int \rho_{1}\left(\mathbf{r}, \mathbf{r}^{\prime}\right) \varphi_{n}\left(\mathbf{r}^{\prime}\right) d \mathbf{r}^{\prime}=\gamma_{n} \varphi_{n}(\mathbf{r}) .
$$

The largest eigenvalue defines the norm

$$
\left\|\hat{\rho}_{1}\right\| \equiv \sup _{n} \gamma_{n}
$$

The Penrose criterion of condensation reads

$$
\lim _{N \rightarrow \infty} \frac{\left\|\hat{\rho}_{1}\right\|}{N}>0
$$

This criterion can be further generalized by introducing the notion of order indices for reduced density matrices (Coleman and Yukalov [264, 265]). For a $k$-order reduced density matrix

$$
\rho_{k}\left(\mathbf{r}_{1} \ldots \mathbf{r}_{k}, \mathbf{r}_{1}^{\prime} \ldots \mathbf{r}_{k}^{\prime}\right) \equiv<\psi^{\dagger}\left(\mathbf{r}_{k}^{\prime}\right) \ldots \psi^{\dagger}\left(\mathbf{r}_{1}^{\prime}\right) \psi\left(\mathbf{r}_{1}\right) \ldots \psi\left(\mathbf{r}_{k}\right)>
$$

the order index is defined as

$$
\alpha_{k} \equiv \lim _{N \rightarrow \infty} \frac{\ln \left\|\hat{\rho}_{k}\right\|}{\ln N}
$$

where $\left\|\hat{\rho}_{k}\right\|$ is the norm of the matrix $\hat{\rho}_{k}$ with elements (7.13). Different types of ordering appearing in the system of bosons can be classified [266, 267] as follows:

$$
\begin{gathered}
\alpha_{k}=0, \quad \text { no order or short-range } \\
0<\alpha_{2 k}<k, \quad \text { even mid-range } \\
\alpha_{2 k}=k, \quad \text { even long-range } \\
{\left[\frac{k}{2}\right]<\alpha_{k}<k, \quad \text { total mid-range }} \\
\alpha_{k}=k, \quad \text { total long-range },
\end{gathered}
$$

where $[x]$ is the entire part of $x$. This classification encompasses three kinds of possible condensation: Even condensation [268]-276], with $\alpha_{2 k}=k$, when the groups of even numbers of atoms are condensed but there is no single-particle condensate. Mid-range condensation [277]-280], with $[k / 2]<\alpha_{k}<k$, when there arises algebraic mid-range order but there is no long-range order. The Bose-Einstein singe-particle condensation corresponds to the case $\alpha_{k}=k$.

Criteria based on the consideration of norms of reduced density matrices are rather general. However, it is not always easy to find the eigenvalues of the density matrices for interacting particles. 


\subsection{Off-Diagonal Long-Range Order}

The concept of off-diagonal long-range order (Yang [281) can be formulated as follows. If the limit

$$
\lim _{r_{12} \rightarrow \infty} \rho_{1}\left(\mathbf{r}_{1}, \mathbf{r}_{2}\right) \equiv \rho_{0}>0
$$

where $r_{12} \equiv\left|\mathbf{r}_{1}-\mathbf{r}_{2}\right|$ is not zero, then there occurs Bose-Einstein condensation, and $\rho_{0}$ is the condensate density. Really, for the first-order density matrix one may write the spectral resolution

$$
\rho_{1}\left(\mathbf{r}_{1}, \mathbf{r}_{2}\right)=\sum_{n} \gamma_{n} \varphi_{n}\left(\mathbf{r}_{1}\right) \varphi_{n}^{*}\left(\mathbf{r}_{2}\right)
$$

in which $\gamma_{n}$ are the eigenvalues and $\varphi_{n}(\mathbf{r})$, the eigenfunctions of $\hat{\rho}_{1}$. Note that $\gamma_{n}$ play the role of the average occupation numbers of the single-particle states labelled by $n$. If one assumes that the considered system is uniform, then the main contribution to the sum (7.17), as $r_{12} \rightarrow \infty$, is made by the term containing the largest eigenvalue $\gamma_{0}$ and the ground-state function $\varphi_{0}(\mathbf{r})=1 / \sqrt{V}$, so that

$$
\rho_{1}\left(\mathbf{r}_{1}, \mathbf{r}_{2}\right) \simeq \gamma_{0} \varphi_{0}\left(\mathbf{r}_{1}\right) \varphi_{0}^{*}\left(\mathbf{r}_{2}\right), \quad r_{12} \rightarrow \infty
$$

Here it is not necessary that $\varphi_{0}$ be the average $\langle\psi\rangle$ of the field operator. To be finite, the limit (7.18) requires that $\gamma_{0} \sim N$. Hence the consideration is reduced to the Penrose criterion of condensation (7.12).

Thus, the long-range order defined by the limit (7.16) is a sufficient condition for the occurrence of condensation in a nonuniform system. But, in general, this is not a necessary condition, and it is not applicable to nonuniform systems. For example, for a system of atoms localized in a confined region, say inside a trap, one has

$$
\lim _{r \rightarrow \infty} \varphi_{n}(\mathbf{r})=0
$$

Therefore,

$$
\lim _{r_{12} \rightarrow \infty} \rho_{1}\left(\mathbf{r}_{1}, \mathbf{r}_{2}\right)=0
$$

irrespectively of the values of $\gamma_{n}$. Condition (7.16) excludes the existence of Bose-Einstein condensation in confined systems.

\subsection{Broken Gauge Symmetry}

The concept of broken gauge symmetry is often used as a sufficient condition for Bose-Einstein condensation. The standard way of breaking gauge symmetry is by means of the Bogolubov prescription [282] for the field operator which is presented as the sum

$$
\psi(\mathbf{r})=\psi_{0}(\mathbf{r})+\tilde{\psi}(\mathbf{r})
$$

of a nonoperator term $\psi_{0}$ and an operator $\tilde{\psi}$ such that

$$
\psi_{0}(\mathbf{r})=\langle\psi(\mathbf{r})>, \quad<\tilde{\psi}(\mathbf{r})>=0
$$

The nonoperator term $\psi_{0}$ corresponds to condensate atoms in a single-particle ground state, while the operator $\tilde{\psi}$ describes atoms outside the condensate. Because of Eqs. $(7.21)$ and $(7.22)$, the statistical average of the field operator

$$
<\psi(\mathbf{r})>\neq 0
$$

is not zero, which manifests the broken gauge symmetry as far as the average $<\psi>$ is now not invariant under the gauge transformation

$$
\psi(\mathbf{r}) \rightarrow e^{i \alpha} \psi(\mathbf{r})
$$

where $\alpha$ is an arbitrary real number.

In order to understand better what are the assumptions under which the prescription (7.21) is valid, it is useful to look attentively at the original ideas of Bogolubov 282, which we shall follow below. Let us select 
an orthonormalized basis $\left\{\varphi_{n}(\mathbf{r})\right\}$ of single-particle states. The field operator can be expanded over the chosen basis as

$$
\psi(\mathbf{r})=\sum_{n} \psi_{n}(\mathbf{r})=\sum_{n} a_{n} \varphi_{n}(\mathbf{r}),
$$

with $a_{n}=\left(\varphi_{n}, \psi\right)$. From the Bose commutation relations

$$
\left[\psi(\mathbf{r}), \psi^{\dagger}\left(\mathbf{r}^{\prime}\right)\right]=\delta\left(\mathbf{r}-\mathbf{r}^{\prime}\right)
$$

one has

$$
\left[a_{m}, a_{n}^{\dagger}\right]=\delta_{m n} .
$$

Define the condensate and noncondensate operators

$$
\psi_{0}(\mathbf{r}) \equiv a_{0} \varphi_{0}(\mathbf{r}), \quad \tilde{\psi}(\mathbf{r}) \equiv \sum_{n \neq 0} a_{n} \varphi_{n}(\mathbf{r}) .
$$

From the commutation relations for $a_{n}$ it follows that

$$
\left[\psi_{0}(\mathbf{r}), \psi_{0}^{\dagger}\left(\mathbf{r}^{\prime}\right)\right]=\varphi_{0}(\mathbf{r}) \varphi_{0}^{*}\left(\mathbf{r}^{\prime}\right) .
$$

For treating $\psi_{0}$ as a nonoperator term, it is necessary that this commutator would be asymptotically small, at least, in the thermodynamic limit, when

$$
N \rightarrow \infty, \quad V \rightarrow \infty, \quad \frac{N}{V} \rightarrow \text { const } .
$$

This is really the case for uniform systems for which one has the basis $\left\{\varphi_{k}(\mathbf{r})\right\}$ formed of plane waves

$$
\varphi_{k}(\mathbf{r})=\frac{1}{\sqrt{V}} e^{i \mathbf{k} \cdot \mathbf{r}} .
$$

Then one gets

$$
\left[\psi_{0}(\mathbf{r}), \psi_{0}^{\dagger}\left(\mathbf{r}^{\prime}\right)\right]=\frac{1}{V} \rightarrow 0 \quad(V \rightarrow \infty) .
$$

However this is not yet sufficient for announcing $\psi_{0}$ a nonoperator term. Consider the operator $a_{0}=\left(\varphi_{0}, \psi\right)$. Taking into account that

$$
\left(\varphi_{0}, \tilde{\psi}\right)=\sum_{n \neq 0} a_{n}\left(\varphi_{0}, \varphi_{n}\right)=0
$$

we have

$$
a_{0}=\left(\varphi_{0}, \psi_{0}\right) .
$$

This shows that if $\psi_{0}$ is not an operator then $a_{0}$ is also such. Hence, one should have $\left[a_{0}, a_{0}^{\dagger}\right]=0$, which contradicts the commutation relation $\left[a_{0}, a_{0}^{\dagger}\right]=1$. Then one needs to make an assumption that the ground state is macroscopically occupied, so that

$$
<a_{0}^{\dagger} a_{0}>\sim N .
$$

Only after this, one can say that the finite value of the commutator $\left[a_{0}, a_{0}^{\dagger}\right]$ is negligibly small as compared to the macroscopic number (7.27),

$$
\frac{\left[a_{0}, a_{0}^{\dagger}\right]}{<a_{0}^{\dagger} a_{0}>} \sim \frac{1}{N} \rightarrow 0 \quad(N \rightarrow \infty)
$$

As is evident, the assumption $(7.27)$ is nothing but the Einstein criterion of condensation (7.7). In this way, the Bogolubov prescription (7.21) presupposes Bose-Einstein condensation. Moreover, this prescription assumes that the condensation occurs solely in one quantum state. In general, the commutator $\left[\psi_{n}, \psi_{n}^{\dagger}\right]$ can become asymptotically small for several states. For instance, the commutator

$$
\left[\psi_{k}(\mathbf{r}), \psi_{k}^{\dagger}\left(\mathbf{r}^{\prime}\right)\right]=\frac{1}{V} e^{i \mathbf{k} \cdot\left(\mathbf{r}-\mathbf{r}^{\prime}\right)} \rightarrow 0
$$


is asymptotically, as $V \rightarrow \infty$, small for any $\mathbf{k}$. Nevertheless, one does not announce that all $\psi_{k}$ are nonoperator terms. Vice versa, all $\psi_{k}$ with $\mathbf{k} \neq 0$ are treated as operators satisfying the standard Bose commutation relations. This means that the Bogolubov prescription segregates one ground-state level that is assumed to be macroscopically occupied, so that relation (7.27) holds true; and all other levels are not occupied macroscopically, so that $<a_{n}^{\dagger} a_{n}>\sim 1$ for $n \neq 0$.

To be practical, the Bogolubov prescription (7.21) requires one more assumption that is always made. One treats $\tilde{\psi}$ as a small perturbation about the mean-field value $\psi_{0}=\langle\psi\rangle$. This is equivalent to the assumption that almost all atoms are condensed,

$$
\frac{N-N_{0}}{N} \ll 1
$$

In this way, breaking gauge symmetry by means of the Bogolubov prescription (7.21) presupposes the existence of Bose-Einstein condensation. This prescription provides a practical tool for calculations under the assumption that almost all atoms are in the condensed state. But breaking gauge symmetry is not necessary for the validity of the Einstein criterion $(7.7)$ or Penrose criterion $(7.12)$, that is, it is not necessary for the existence of Bose-Einstein condensation.

It is also important to note that the Bogolubov prescription (7.21) is not applicable for strongly interacting particles whose interactions are described by nonintegrable potentials. Breaking gauge symmetry by this prescription requires that the interatomic interactions are given by an integrable potential $\Phi(\mathbf{r})$, such that

$$
\left|\int \Phi(\mathbf{r}) d \mathbf{r}\right|<\infty \text {. }
$$

This is necessary since using the prescription (7.21) yields the appearance in the Hamiltonian of the term

$$
\frac{1}{2} \int\left|\psi_{0}(\mathbf{r})\right|^{2} \Phi\left(\mathbf{r}-\mathbf{r}^{\prime}\right)\left|\psi_{0}\left(\mathbf{r}^{\prime}\right)\right|^{2} d \mathbf{r} d \mathbf{r}^{\prime},
$$

with nonoperator functions $\left|\psi_{0}(\mathbf{r})\right|$ and $\left|\psi_{0}\left(\mathbf{r}^{\prime}\right)\right|$. This term diverges if the interaction potential does not satisfy condition $(\overline{7.30})$, hence, gauge symmetry cannot be broken for such systems.

\subsection{Condensation in Confined Systems}

When atoms are confined in a box or by means of external confining potentials, then all single-particle functions $\varphi_{n}(\mathbf{r})$ tend to zero, as $r \rightarrow \infty$, because of which the limit (7.20) of the first-order density matrix is zero, which tells that there is no off-diagonal long-range order. However, it is possible to weaken condition (7.20) considering, instead of the exact limit, an asymptotic behavior at large $r_{12}$, when the density matrix can be approximately factorized as

$$
\rho_{1}\left(\mathbf{r}_{1}, \mathbf{r}_{2}\right) \sim \varphi_{0}\left(\mathbf{r}_{1}\right) \varphi_{0}^{*}\left(\mathbf{r}_{2}\right),
$$

which tells that there exists a kind of long-range order [262, 263, 283]. The factorization (7.31) may appear if the distance $r_{12}$ is much larger than the mean interatomic distance $a$, but much smaller than the effective size $l_{0}$ of the confined system, that is, in the region

$$
a \ll r_{12} \ll l_{0} .
$$

The meaning of the inequality $r_{12} \gg a$ is evident, and the inequality $r_{12} \ll l_{0}$ arises because the wave function of a ground state is always more localized than the wave functions of excited states. Consequently, at the distance $r_{12} \sim l_{0}$ the excited-state wave functions are much larger than $\varphi_{0}$, and the factorization (7.31), in general, will not occur. Thus, in confined systems, strictly speaking, there is no long-range order but there can be quasilong-range order, when the density matrix factorizes, as in Eq. (7.31), in the region (7.32).

Gauge symmetry in a confined system cannot be broken. Thus, for employing the Bogolubov prescription (7.21), one would need that the commutator $\left[\psi_{0}, \psi_{0}^{+}\right]$be zero. However, this is not so. For instance, expanding the field operator, according to Eq. (7.24), over an oscillator basis, we have

$$
\varphi_{0}(\mathbf{r})=a_{0}\left(\frac{m_{0} \omega_{0}}{\pi \hbar}\right)^{3 / 4} \exp \left(-\frac{m_{0} \omega_{0}}{2 \hbar} r^{2}\right)
$$


from where

$$
\left[\varphi_{0}\left(\mathbf{r}_{1}\right), \psi_{0}^{\dagger}\left(\mathbf{r}_{2}\right)\right]=\left(\frac{m_{0} \omega_{0}}{\pi \hbar}\right)^{3 / 2} \exp \left\{-\frac{m_{0} \omega_{0}}{2 \hbar}\left(r_{1}^{2}+r_{2}^{2}\right)\right\}
$$

which is, certainly, not zero.

The absence of long-range order and of broken gauge symmetry in confined systems is in agreement with the known fact that there are no sharp phase transitions in such systems, although Bose-Einstein condensation can occur without being a sharp phase transition but a gradual crossover [41, 284- 287. During this crossover all thermodynamic characteristics behave smoothly and no discontinuities appear, although some quantities can change very rapidly. Since all thermodynamic characteristics change in a completely smooth way, the identification of a specific critical temperature is problematic. It is the standard situation for crossover phenomena that the crossover temperature is not uniquely defined, but its definition, anyway, can be done by assigning the crossover temperature to the maximum of one of thermodynamic functions [288, 289]. In the case of Bose condensation in confined systems, one can relate the condensation temperature to the maximum of specific heat 41.

If gauge symmetry in confined systems is, strictly speaking, never broken, is it then admissible to use the Bogolubov prescription (7.21) in some approximate sense? It seems that when the mean interatomic distance $a$ is much smaller than the effective system size $l_{0}$, then the confined system could be treated as almost infinite. For atoms confined, e.g. in a harmonic potential, the required inequality is

$$
\frac{a}{l_{0}} \ll 1, \quad l_{0} \equiv \sqrt{\frac{\hbar}{m_{0} \omega_{0}}} .
$$

The effective volume of the confined system is $V \sim l_{0}^{3}$, hence $l_{0} \sim N^{1 / 3}$. Therefore, an admissible description of the effective thermodynamic limit in this case could be

$$
N \rightarrow \infty, \quad l_{0} \rightarrow \infty, \quad \frac{N}{l_{0}^{3}} \rightarrow \text { const } .
$$

Because of the relation (7.34), $\omega_{0} \sim l_{0}^{-2}$, thence $\omega_{0} \sim N^{-2 / 3}$. Consequently, the thermodynamic limit (7.35) can be presented as

$$
N \rightarrow \infty, \quad \omega_{0} \rightarrow 0, \quad N \omega_{0}^{3 / 2} \rightarrow \text { const } .
$$

The ground-state wave function $\varphi_{0} \sim l_{0}^{-3 / 2}$, that is $\varphi_{0} \sim N^{-1 / 2}$. Then the commutator (7.33) is

$$
\left[\psi_{0}\left(\mathbf{r}_{1}\right), \psi_{0}^{\dagger}\left(\mathbf{r}_{2}\right)\right] \sim \frac{1}{\pi^{3 / 2} l_{0}^{3}} \sim \frac{1}{N}
$$

which shows that it is asymptotically zero, as $N \rightarrow \infty$, for any $\mathbf{r}_{1}$ and $\mathbf{r}_{2}$. This means that the Bogolubov prescription (7.21) can have the sense of an approximate relation for large confined systems satisfying condition (7.34). A slightly different definition of the effective thermodynamic limit for trapped atoms will be given in Section 11. It is worth recalling that breaking gauge symmetry is a sufficient condition for Bose-Einstein condensation but not necessary 290. The Einstein criterion (7.7) or Penrose criterion (7.12) do not require any broken symmetry. The occurrence of Bose-Einstein condensation in a confined system, say in a trap, can be noticed by observing the density of atoms, which can be presented as the sum

$$
\rho(\mathbf{r})=\rho_{0}(\mathbf{r})+\tilde{\rho}(\mathbf{r}),
$$

where the first and second terms correspond to the density of atoms in a ground state and in excited states, respectively,

$$
\rho_{0}(\mathbf{r}) \equiv N_{0}\left|\varphi_{0}(\mathbf{r})\right|^{2}, \quad \tilde{\rho}(\mathbf{r}) \equiv \sum_{n \neq 0} N_{n}\left|\varphi_{n}(\mathbf{r})\right|^{2} .
$$

According to the Einstein or Penrose criteria, condensation happens when $N_{0} \sim N$, which does not involve any mentioning of gauge symmetry. In experiments, the occurrence of condensation is manifested by the appearance 
of a narrow distribution $\rho_{0}(\vec{r})$ above the wider $\tilde{\rho}(\mathbf{r})$. Because of the normalization

$$
\int \rho(\mathbf{r}) d \mathbf{r}=N, \quad N_{0}+\sum_{n \neq 0} N_{n}=N,
$$

the ground-state density $\rho_{0}(\mathbf{r})$ becomes noticeable when $N_{0} \sim N$. Although condensation in a trap is a gradual crossover, the latter can be rather sharp reminding a phase transition occurring at a point. 


\section{Chapter 8}

\section{Coherent Atomic States}

One usually connects the occurrence of Bose-Einstein condensation with the appearance of coherence in an atomic system. This sounds reasonable since inequalities (7.1) and (7.3) can be interpreted as the conditions of coherence. In order to understand better the relation between condensation and coherence, it is necessary to give a rigorous definition of coherent states and to study their main properties. This is done in the following subsections that are based on Ref. 291.

\subsection{Definition and Main Properties}

We consider the field operators $\psi(\mathbf{r})$ and $\psi^{\dagger}(\mathbf{r})$ satisfying the Bose commutation relations and defined on the Fock space $\mathcal{F}$. A state $h \in \mathcal{F}$ is called a coherent state if it is an eigenvector of the annihilation operator

$$
\psi(\mathbf{r}) h=\eta(\mathbf{r}) h .
$$

The function, $\eta(\mathbf{r})$, playing the role of an eigenvalue, can be called the coherent field. The latter is assumed to be normalizable with the norm $\|\eta\| \equiv(\eta, \eta)$ defined by means of the scalar product

$$
\left(\eta, \eta^{\prime}\right) \equiv \int \eta^{*}(\mathbf{r}) \eta^{\prime}(\mathbf{r}) d \mathbf{r}
$$

The coherent state $h$ is not an eigenvector of the creation operator. But there is a useful property

$$
h^{+} \psi^{\dagger}(\mathbf{r})=\eta^{*}(\mathbf{r}) h^{+}
$$

that follows from the Hermitian conjugation of Eq. (8.1). The state $h$, being a vector of the Fock space $\mathcal{F}$, is presentable as a column

$$
h=\left\{h_{k}\left(\mathbf{r}_{1}, \mathbf{r}_{2}, \ldots, \mathbf{r}_{k}\right) \mid k=0,1,2, \ldots\right\} .
$$

From the definition 8.1) one can derive that

$$
h_{k}\left(\mathbf{r}_{1}, \mathbf{r}_{2}, \ldots, \mathbf{r}_{k}\right)=\frac{C_{0}}{\sqrt{k !}} \prod_{j=1}^{k} \eta\left(\mathbf{r}_{j}\right) .
$$

Requiring that the state (8.3) be normalized to unity,

$$
h^{+} h=\sum_{i=1}^{\infty}\left(h_{i}, h_{i}\right)=1,
$$

where

$$
\left(h_{i}, h_{i}\right) \equiv \int\left|h_{i}\left(\mathbf{r}_{1}, \mathbf{r}_{2}, \ldots, \mathbf{r}_{i}\right)\right|^{2} d \mathbf{r}_{1} d \mathbf{r}_{2} \ldots d \mathbf{r}_{i}
$$


one gets the normalization constant

$$
\left|C_{0}\right|=\exp \left\{-\frac{1}{2}(\eta, \eta)\right\} .
$$

Two different coherent states are not orthogonal since the product

$$
h^{+} h^{\prime}=\exp \left\{-\frac{1}{2}(\eta, \eta)+\left(\eta, \eta^{\prime}\right)-\frac{1}{2}\left(\eta^{\prime}, \eta^{\prime}\right)\right\}
$$

is not zero.

It is possible to introduce time-dependent coherent states

$$
h(t)=\hat{U}(t) h
$$

by means of the evolution operator $\hat{U}(t)$, which is a unitary operator satisfying the Schrödinger equation

$$
i \hbar \frac{d}{d t} \hat{U}(t)=H \hat{U}(t)
$$

where $H$ is a Hamiltonian depending, in general, on time. The generalization of definition (8.1) is the eigenproblem

$$
\psi(\mathbf{r}) h(t)=\eta(\mathbf{r}, t) h(t) .
$$

Similarly to the time-independent case, one may derive that $h(t)$ has the structure of the column

$$
h(t)=\left\{\frac{C_{0}(t)}{\sqrt{k !}} \prod_{j=1}^{k} \eta\left(\mathbf{r}_{j}, t\right)\right\} .
$$

The eigenproblem (8.9) can also be presented in the form

$$
\psi(\mathbf{r}, t) h=\eta(\mathbf{r}, t) h
$$

involving the time-dependent field operator

$$
\psi(\mathbf{r}, t)=\hat{U}^{+}(t) \psi(\mathbf{r}) \hat{U}(t) .
$$

If the evolution of the system is prescribed by the Schrödinger equation (8.8), then the coherent field $\eta(\mathbf{r}, t)$ is not arbitrary. Let us take the system Hamiltonian in the standard form

$$
\begin{gathered}
H=\int \psi^{\dagger}(\mathbf{r}, t)\left[-\frac{\hbar^{2} \nabla^{2}}{2 m_{0}}+U(\mathbf{r}, t)\right] \psi(\mathbf{r}, t) d \mathbf{r}+ \\
+\frac{1}{2} \int \psi^{\dagger}(\mathbf{r}, t) \psi^{\dagger}\left(\mathbf{r}^{\prime}, t\right) \Phi\left(\mathbf{r}-\mathbf{r}^{\prime}\right) \psi\left(\mathbf{r}^{\prime}, t\right) \psi(\mathbf{r}, t) d \mathbf{r} d \mathbf{r}^{\prime},
\end{gathered}
$$

with the interaction potential $\Phi(-\mathbf{r})=\Phi(\mathbf{r})$. The evolution prescribed by Eqs. (8.8) and (8.12) yields the Heisenberg equation

$$
i \hbar \frac{\partial}{\partial t} \psi(\mathbf{r}, t)=[\psi(\mathbf{r}, t), H],
$$

which is also equivalent to the variational equation

$$
i \hbar \frac{\partial \psi(\mathbf{r}, t)}{\partial t}=\frac{\delta H}{\delta \psi^{\dagger}(\mathbf{r}, t)}
$$

With the Hamiltonian (8.13), the evolution equation for the field operator (8.12) is

$$
i \hbar \frac{\partial}{\partial t} \psi(\mathbf{r}, t)=H(\psi) \psi(\mathbf{r}, t),
$$


where

$$
H(\psi) \equiv-\frac{\hbar^{2} \nabla^{2}}{2 m_{0}}+U(\mathbf{r}, t)+\int \Phi\left(\mathbf{r}-\mathbf{r}^{\prime}\right) \psi^{\dagger}\left(\mathbf{r}^{\prime}, t\right) \psi\left(\mathbf{r}^{\prime}, t\right) d \mathbf{r}^{\prime} .
$$

Multiplying Eq. (8.14) by $h^{+}$from the left and by $h$ from the right, and using definition 8.11), yields the evolution equation for the coherent field

$$
i \hbar \frac{\partial}{\partial t} \eta(\mathbf{r}, t)=H(\eta) \eta(\mathbf{r}, t)
$$

with the effective nonlinear Hamiltonian

$$
H(\eta)=-\frac{\hbar^{2} \nabla^{2}}{2 m_{0}}+U(\mathbf{r}, t)+\int \Phi\left(\mathbf{r}-\mathbf{r}^{\prime}\right)\left|\eta\left(\mathbf{r}^{\prime}, t\right)\right|^{2} d \mathbf{r}^{\prime} .
$$

The nonlinear Schrödinger equation (8.16) is the exact equation for the coherent field.

The norm of the coherent field has not yet been specified and, in general, it can be arbitrary. It is convenient to introduce the coherent field $\varphi(\mathbf{r}, t)$ normalized to unity, so that

$$
\eta(\mathbf{r}, t) \equiv \sqrt{\kappa} \varphi(\mathbf{r}, t),
$$

where $\kappa$ is an arbitrary positive number and

$$
(\eta, \eta)=\kappa, \quad(\varphi, \varphi)=1
$$

Then Eqs. 8.16) and (8.17) are transformed to the nonlinear Schrödinger equation

$$
i \hbar \frac{\partial}{\partial t} \varphi(\mathbf{r}, t)=\hat{H}(\varphi) \varphi(\mathbf{r}, t)
$$

with the nonlinear Hamiltonian

$$
\hat{H}(\varphi) \equiv-\frac{\hbar^{2} \nabla^{2}}{2 m_{0}}+U(\mathbf{r}, t)+\kappa \int \Phi\left(\mathbf{r}-\mathbf{r}^{\prime}\right)\left|\varphi\left(\mathbf{r}^{\prime}, t\right)\right|^{2} d \mathbf{r}^{\prime} .
$$

Notice that Eq. 8.19) has nontrivial solutions for the coherent field only if the interaction potential is integrable in the sense of condition (7.30). A system of particles with a nonintegrable interaction potential cannot possess coherent states.

\subsection{Stationary Coherent States}

If the external potential $U(\mathbf{r}, t)=U(\mathbf{r})$ does not depend on time, then the nonlinear Schrödinger equation 8.19) has stationary solutions of the form

$$
\varphi_{n}(\mathbf{r}, t)=\varphi_{n}(\mathbf{r}) \exp \left(-\frac{i}{\hbar} E_{n} t\right)
$$

in which $\varphi_{n}(\mathbf{r})$ and $E_{n}$ are defined by the eigenproblem

$$
\hat{H}\left(\varphi_{n}\right) \varphi_{n}(\mathbf{r})=E_{n} \varphi_{n}(\mathbf{r}) .
$$

The stationary solutions $\varphi_{n}$ labelled by a multi-index $n$ can be called coherent modes.

The Hamiltonian (8.20) is nonlinear, hence cannot be Hermitian. Therefore the set of solutions $\left\{\varphi_{n}(\mathbf{r})\right\}$ to the eigenproblem (8.22) does not necessarily form a complete orthonormal basis. Actually, even in the case of a Hermitian operator in an infinite-dimensional space, the set of its eigenvectors does not always form such a basis 292, 293], contrary to the case of Hermitian operators in finite-dimensional spaces. 
Nonlinear eigenproblems are usually solved by an iterative procedure, as it is done for self-consistent meanfield problems like Hartree or Hartree-Fock equations [294, 295]. In the process of such solutions it is often possible to preserve the orthogonality of eigenvectors, at least approximately in the sense of the inequality

$$
\left|\left(\varphi_{m}, \varphi_{n}\right)\right| \ll 1, \quad m \neq n .
$$

The latter, because of Eq. (8.22), is equivalent to the condition

$$
\left|\left(\hat{H} \varphi_{m}, \varphi_{n}\right)-\left(\varphi_{m}, \hat{H} \varphi_{n}\right)\right| \ll\left|E_{m}-E_{n}\right|,
$$

if $m \neq n$ and $\hat{H} \varphi \equiv \hat{H}(\varphi) \varphi$. Here, with the Hamiltonian (8.20), we have

$$
\begin{gathered}
\left(\hat{H} \varphi_{m}, \varphi_{n}\right)-\left(\varphi_{m}, \hat{H} \varphi_{n}\right)= \\
=\kappa \int \varphi_{m}^{*}(\mathbf{r}) \Phi\left(\mathbf{r}-\mathbf{r}^{\prime}\right)\left[\left|\varphi_{m}\left(\vec{r}^{\prime}\right)\right|^{2}-\left|\varphi_{n}\left(\mathbf{r}^{\prime}\right)\right|^{2}\right] \varphi_{n}(\mathbf{r}) d \mathbf{r} d \mathbf{r}^{\prime} .
\end{gathered}
$$

Thus, the set $\left\{\varphi_{n}\right\}$, in general, is not orthogonal although can often be made quasiorthogonal, so that $\mid\left(\varphi_{m}, \varphi_{n}\right) \ll$ 1 if $m \neq n$. The modes $\varphi_{n}$ can always be normalized to $\left\|\varphi_{n}\right\|=1$.

One may notice that the eigenproblem (8.22), with the Hamiltonian 8.20 ), defines the coherent modes up to a phase factor $e^{i \alpha}$ with an arbitrary real phase $\alpha$. Therefore, the general solution of the eigenproblem (8.22) writes

$$
\varphi_{n \alpha}(\mathbf{r})=\varphi_{n}(\mathbf{r}) e^{i \alpha} \quad(0 \leq \alpha \leq 2 \pi) .
$$

The phase $\alpha$ is an unobservable random variable uniformly distributed in the interval $[0,2 \pi)$, which has to be averaged over when evaluating the expectation values of observables 296]-298]. The random global phase $\alpha$ should not be confused with a local phase of the coherent mode $\varphi_{n}$, which can arise in the process of solution of the eigenproblem (8.22) and which is determined by this eigenproblem.

Keeping all numbers $\kappa, n$, and $\alpha$ fixed, we have a pure coherent mode

$$
\eta_{\kappa n \alpha}=\sqrt{\kappa} \varphi_{n}(\mathbf{r}) e^{i \alpha} .
$$

Then, Eqs. (8.3) and (8.4) define a pure coherent state

$$
h_{\kappa n \alpha}=\left\{\frac{C_{0}}{\sqrt{k !}} \prod_{j=1}^{k} \eta_{\kappa n \alpha}\left(\mathbf{r}_{j}\right)\right\},
$$

in which

$$
\left|C_{0}\right|=\exp \left(-\frac{1}{2} \kappa\right) .
$$

The pure coherent states are not orthogonal since

$$
h_{\kappa n \alpha}^{+} h_{\kappa^{\prime} n^{\prime} \alpha^{\prime}}=\exp \left\{-\frac{1}{2}\left(\kappa+\kappa^{\prime}\right)+\sqrt{\kappa \kappa^{\prime}}\left(\varphi_{n}, \varphi_{n^{\prime}}\right) e^{-i\left(\alpha-\alpha^{\prime}\right)}\right\} .
$$

But they are asymptotically orthogonal if either $\kappa \rightarrow \infty$ or $\kappa^{\prime} \rightarrow \infty$. For example, if the pure coherent modes (8.24) are normalized to the number of particles $N=\kappa=\kappa^{\prime}$, then the product 8.26 ) is

$$
h_{N n \alpha}^{+} h_{N n^{\prime} \alpha^{\prime}}=\exp \left\{-N+\left(\varphi_{n}, \varphi_{n^{\prime}}\right) N e^{i\left(\alpha^{\prime}-\alpha\right)}\right\} .
$$

This shows that two different pure states, for which either $n \neq n^{\prime}$ or $\alpha \neq \alpha^{\prime}$, are asymptotically orthogonal in the sense of the limit

$$
\lim _{N \rightarrow \infty} h_{N n \alpha}^{+} h_{N n^{\prime} \alpha^{\prime}}=\delta_{n n^{\prime}} \delta_{\alpha \alpha^{\prime}} .
$$

Thus, the set $\left\{h_{N n \alpha}\right\}$ of pure coherent states forms a normalized asymptotically, with respect to $N \rightarrow \infty$, orthogonal basis. This basis is asymptotically, as $N \rightarrow \infty$, completely providing the resolution of unity

$$
\sum_{n} h_{N n \alpha} h_{N n \alpha}^{+} \simeq \hat{1} \quad(N \rightarrow \infty) .
$$


The latter equality is to be understood in the weak sense as an equality for the matrix elements

$$
h_{N n \alpha}^{+} \hat{1} h_{N n^{\prime} \alpha^{\prime}} \simeq \delta_{n n^{\prime}} \delta_{\alpha \alpha^{\prime}} \quad(N \rightarrow \infty) .
$$

Hence the set $\left\{h_{N n \alpha}\right\}$ can be treated as an asymptotically complete and orthonormalized basis, when $N \rightarrow \infty$.

\subsection{Quantum Coherent Averages}

For an operator $\hat{A}$, we can define the pure coherent average

$$
<\hat{A}>_{\kappa n \alpha} \equiv h_{\kappa n \alpha}^{+} \hat{A} h_{\kappa n \alpha}
$$

with respect to the pure coherent states (8.25). Thus for the field operator, one has

$$
<\psi(\mathbf{r})>_{\kappa n \alpha}=\sqrt{\kappa} \varphi_{n}(\mathbf{r}) e^{i \alpha},
$$

which tells us that the usage of pure coherent states breaks gauge symmetry. The first-order density matrix factorizes as

$$
<\psi^{\dagger}(\mathbf{r}) \psi\left(\mathbf{r}^{\prime}\right)>_{\kappa n \alpha}=<\psi^{\dagger}(\mathbf{r})>_{\kappa n \alpha}<\psi\left(\mathbf{r}^{\prime}\right)>_{\kappa n \alpha} .
$$

For the particle density operator

$$
\hat{n}(\mathbf{r}) \equiv \psi^{\dagger}(\mathbf{r}) \psi(\mathbf{r})
$$

one gets

$$
<\hat{n}(\mathbf{r})>_{\kappa n \alpha}=\kappa\left|\varphi_{n}(\mathbf{r})\right|^{2} .
$$

And the density-density correlation function is

$$
<\hat{n}(\mathbf{r}) \hat{n}\left(\mathbf{r}^{\prime}\right)>_{\kappa n \alpha}=<\hat{n}(\mathbf{r})>_{\kappa n \alpha}<\hat{n}\left(\mathbf{r}^{\prime}\right)>_{\kappa n \alpha}+\delta\left(\mathbf{r}-\mathbf{r}^{\prime}\right)<\hat{n}(\mathbf{r})>_{\kappa n \alpha} .
$$

This function, in general, is not factorized. However, there is no great sense to consider correlations at one point. It is meaningful to consider the correlations only for $\mathbf{r} \neq \mathbf{r}^{\prime}$. In the latter case, the correlation function (8.35) is factorized.

The average number of particles is

$$
<\hat{N}>_{\kappa n \alpha}=\kappa, \quad \hat{N} \equiv \int \hat{n}(\mathbf{r}) d \mathbf{r} .
$$

And the average of the Hamiltonian (8.13) writes

$$
\begin{aligned}
<H & >_{\kappa n \alpha}=\kappa \int \varphi_{n}^{*}(\mathbf{r})\left[-\frac{\hbar^{2} \nabla^{2}}{2 m_{0}}+U(\mathbf{r})\right] \varphi_{n}(\mathbf{r}) d \mathbf{r}+ \\
& +\frac{\kappa^{2}}{2} \int\left|\varphi_{n}(\mathbf{r})\right|^{2} \Phi\left(\mathbf{r}-\mathbf{r}^{\prime}\right)\left|\varphi_{n}\left(\mathbf{r}^{\prime}\right)\right|^{2} d \mathbf{r} d \mathbf{r}^{\prime},
\end{aligned}
$$

where a stationary external potential is assumed. Employing the eigenproblem (8.22), the average energy (8.37) can be rewritten in two other forms,

$$
\begin{aligned}
& <H>_{\kappa n \alpha}=\kappa E_{n}-\frac{\kappa^{2}}{2} \int\left|\varphi_{n}(\mathbf{r})\right|^{2} \Phi\left(\mathbf{r}-\mathbf{r}^{\prime}\right)\left|\varphi_{n}\left(\mathbf{r}^{\prime}\right)\right|^{2} d \mathbf{r} d \mathbf{r}^{\prime}, \\
& <H>_{\kappa n \alpha}=\frac{\kappa}{2} E_{n}+\frac{\kappa}{2} \int \varphi_{n}^{*}(\mathbf{r})\left[-\frac{\hbar^{2} \nabla^{2}}{2 m_{0}}+U(\mathbf{r})\right] \varphi_{n}(\mathbf{r}) d \mathbf{r} .
\end{aligned}
$$

As has been explained above, the phase $\alpha$ in the coherent modes (8.23) and (8.24) is an unobservable random variable that has to be averaged out when calculating the expectation values of operators. This means that the 
pure coherent averages (8.31), strictly speaking, do not correspond to observable quantities. The latter are to be defined as the averages over the random-phase coherent state 296]

$$
h_{\kappa n} \equiv\left\{h_{\kappa n \alpha} \mid \alpha \in[0,2 \pi)\right\}
$$

being the set of all pure states $h_{\kappa n \alpha}$, with the random variable $\alpha$. The corresponding coherent average is

$$
<\hat{A}>_{\kappa n} \equiv \frac{1}{2 \pi} \int_{0}^{2 \pi}<\hat{A}>_{\kappa n \alpha} d \alpha .
$$

Then, for instance, for the field operator one has

$$
<\psi(\mathbf{r})>_{\kappa n}=0,
$$

which shows that for the coherent state (8.39) gauge symmetry is not broken. This sounds rather reasonable since the field operator does not pertain to the algebra of observables [291, 299. One more reason for the absence of broken gauge symmetry is that Eq. (8.41) is in agreement with the conservation of the number of particles [300]. The absence of broken gauge symmetry does not preclude the first-order density matrix from being factorized as

$$
<\psi^{\dagger}(\mathbf{r}) \psi\left(\mathbf{r}^{\prime}\right)>_{\kappa n}=\kappa \varphi_{n}^{*}(\mathbf{r}) \varphi_{n}\left(\mathbf{r}^{\prime}\right) .
$$

But the form (8.33) does not hold true,

$$
<\psi^{\dagger}(\mathbf{r}) \psi\left(\mathbf{r}^{\prime}\right)>_{\kappa n} \neq<\psi^{\dagger}(\mathbf{r})>_{\kappa n}<\psi\left(\mathbf{r}^{\prime}\right)>_{\kappa n},
$$

because of Eq. 8.41).

The operators $A$ pertaining to the algebra of observables contain the products of even numbers of field operators, with equal numbers of creation and annihilation operators. Therefore, for such operators, the coherent averages (8.40) coincide with the pure averages (8.31). For example, the average particle density

$$
<\hat{n}(\mathbf{r})>_{\kappa n}=\kappa\left|\varphi_{n}(\mathbf{r})\right|^{2}
$$

is the same as the density (8.34). The density-density correlation function

$$
<\hat{n}(\mathbf{r}) \hat{n}\left(\mathbf{r}^{\prime}\right)>_{\kappa n}=<\hat{n}(\mathbf{r})>_{\kappa n}<\hat{n}\left(\mathbf{r}^{\prime}\right)>_{\kappa n}+\delta\left(\mathbf{r}-\mathbf{r}^{\prime}\right)<\hat{n}(\mathbf{r})>_{\kappa n}
$$

is analogous to that of (8.35). Again considering the correlations for different points $\mathbf{r} \neq \mathbf{r}^{\prime}$, we see that the correlator (8.44) factorizes. One may also notice that the second term in the right-hand side of Eq. (8.44) can be omitted when $\kappa \gg 1$, since on average it is much smaller than the first term. This follows from the integration

$$
\int<\hat{n}(\mathbf{r}) \hat{n}\left(\mathbf{r}^{\prime}\right)>_{\kappa n} d \mathbf{r} d \mathbf{r}^{\prime}=\kappa^{2}+\kappa
$$

For any two operators $\hat{A}(\mathbf{r})$ and $\hat{B}\left(\mathbf{r}^{\prime}\right)$, the correlator defined through the coherent average (8.40) always factorizes if $\mathbf{r} \neq \mathbf{r}^{\prime}$. When both these operators pertain to the algebra of observables, the factorization takes the form

$$
<\hat{A}(\mathbf{r}) \hat{B}\left(\mathbf{r}^{\prime}\right)>_{\kappa n}=<\hat{A}(\mathbf{r})>_{\kappa n}<\hat{B}\left(\mathbf{r}^{\prime}\right)>_{\kappa n},
$$

if $\mathbf{r} \neq \mathbf{r}^{\prime}$. However, if one of these operators is not from the algebra of observables, then the correlator does not have the form (8.45), although the factorization does occur. For instance, the density matrix (8.42) is factorized, though $\varphi_{n}(\mathbf{r})$ is not proportional to $\left\langle\psi(\mathbf{r})>_{\kappa n}\right.$, which is zero according to Eq. (8.41). The factorization properties of the coherent averages (8.40) are not connected with gauge symmetry breaking. The latter occurs only for the pure average (8.31), with a fixed global phase. However, it looks unphysical to fix a phase that is random and cannot be measured. 


\subsection{Statistical Coherent Averages}

For a system of many particles, the statistical state is presented by a given statistical operator $\hat{\rho}$ defining the expectation values of operators as statistical averages

$$
<\hat{A}>=\operatorname{Tr} \hat{\rho} \hat{A} .
$$

Since the trace operation does not depend on the chosen basis, we may take for this purpose the basis $\left\{h_{\kappa n \alpha}\right\}$ of the coherent states (8.25). Then the statistical average (8.46) is presented as

$$
<\hat{A}>=\sum_{n} \int_{0}^{\infty} \int_{0}^{2 \pi}<\hat{\rho} \hat{A}>_{\kappa n \alpha} d \kappa d \alpha,
$$

where $\langle\ldots\rangle_{\kappa n \alpha}$ is the pure coherent average (8.31). For an equilibrium statistical state, the Gibbs statistical operator is

$$
\hat{\rho}=\frac{\exp \{-\beta(H-\mu \hat{N})\}}{\operatorname{Tr} \exp \{-\beta(H-\mu \hat{N})\}},
$$

where $\beta \equiv\left(k_{B} T\right)^{-1}$ and the chemical potential $\mu$ can be found from the condition $\langle\hat{N}\rangle=N$. The statistical operator (8.48) represents the grand canonical Gibbs ensemble.

In the thermodynamic limit, $N \rightarrow \infty$, the coherent states become sharply peaked around the average number of particles [301]. If we make a reasonable assumption that, integrating over the norm $\kappa \equiv\|\eta\|$ in the average (8.47), the main contribution, when $N \rightarrow \infty$, comes from the term with $\kappa=N$, then the value (8.47) is asymptotically close to the statistical coherent average

$$
<\hat{A}>_{N} \equiv \sum_{n} \int_{0}^{2 \pi}<\hat{\rho} \hat{A}>_{N n \alpha} d \alpha
$$

defined as a trace over the restricted Hilbert space

$$
\mathcal{H}_{N} \equiv \mathcal{L}\left\{h_{N n \alpha}\right\}
$$

being a linear envelope of the coherent basis $\left\{h_{N n \alpha}\right\}$. Since the observable quantities

$$
<\hat{N}>_{N n \alpha}=N, \quad<H>_{N n \alpha} \equiv E_{N n}
$$

do not depend on the unobservable random phase $\alpha$, and because of the asymptotic orthogonality (8.28), the average (3.49) can be written as

$$
<\hat{A}>_{N} \simeq \sum_{n} \rho_{N n}<\hat{A}>_{N n},
$$

with the coherent statistical weight

$$
\rho_{N n} \equiv \frac{\exp \left(-\beta E_{N n}\right)}{\sum_{n} \exp \left(-\beta E_{N n}\right)}
$$

and with the coherent average

$$
<\hat{A}>_{N n} \equiv \frac{1}{2 \pi} \int_{0}^{2 \pi}<\hat{A}>_{N n \alpha} d \alpha .
$$

The averaged field operator is zero,

$$
<\psi(\mathbf{r})>_{N}=0,
$$

showing that gauge symmetry is not broken. The density matrix

$$
<\psi^{\dagger}(\mathbf{r}) \psi\left(\mathbf{r}^{\prime}\right)>_{N} \simeq N \sum_{n} \rho_{N n} \varphi_{n}^{*}(\mathbf{r}) \varphi_{n}\left(\mathbf{r}^{\prime}\right)
$$


does not factorize as well as the density-density correlation function

$$
<\hat{n}(\mathbf{r}) \hat{n}\left(\mathbf{r}^{\prime}\right)>_{N} \simeq N^{2} \sum_{n} \rho_{N n}\left|\varphi_{n}(\mathbf{r})\right|^{2}\left|\varphi_{n}\left(\mathbf{r}^{\prime}\right)\right|^{2} .
$$

But as temperature tends to zero, and $\beta \rightarrow \infty$, then the system tends to the ground-state energy level

$$
E_{N 0} \equiv \min _{n} E_{N n}
$$

so that the weight 8.53 ) becomes

$$
\rho_{N n} \rightarrow \delta_{n 0} \quad(\beta \rightarrow \infty)
$$

Then the correlators 8.56 ) and (8.57) asymptotically factorize. For example, the density-density correlator is

$$
<\hat{n}(\mathbf{r}) \hat{n}\left(\mathbf{r}^{\prime}\right)>_{N} \simeq<\hat{n}(\mathbf{r})>_{N}<\hat{n}\left(\mathbf{r}^{\prime}\right)>_{N},
$$

when $\beta \rightarrow \infty$. The atom density

$$
<\hat{n}(\mathbf{r})>_{N} \simeq N \sum_{n} \rho_{N n}\left|\varphi_{n}(\mathbf{r})\right|^{2}
$$

can be written as

$$
<\hat{n}(\mathbf{r})>_{N} \simeq N \rho_{N 0}\left|\varphi_{0}(\mathbf{r})\right|^{2}+N \sum_{n \neq 0}\left|\varphi_{n}(\mathbf{r})\right|^{2} .
$$

At high temperatures, when the first term in Eq. 8.60 is negligible, one may say that the system is in thermal state. The temperature $T_{c}$, at which the coherent first term becomes noticeable, characterizes the transition to the coherent state. Since the ground-state wave function is better localized in space than the wave functions of excited modes, the increase of the first term in the sum (8.60) can be noticed as the appearance of a narrow space distribution above the wide thermal cloud described by the second term. With lowering temperature below $T_{c}$, the sharp coherent peak described by the first term grows while the wide thermal distribution corresponding to the second term diminishes. At zero temperature, all atoms are concentrated in the ground-state coherent mode. In this way, Bose-Einstein condensation can be understood as a transition of atoms from excited single-state coherent modes to the ground-state coherent mode.

Note that the coherent states 8.25 ) and 8.39 ) are not the eigenvectors of the system Hamiltonian (8.13). However, this does not preclude the statistical average (8.46) from satisfying the limiting relation

$$
\lim _{N \rightarrow \infty} \lim _{\beta \rightarrow \infty} \frac{1}{N}<\hat{A}>=\lim _{N \rightarrow \infty} \frac{1}{N}<\hat{A}>_{N 0} .
$$

And there is no contradiction between the many-particle coherent states (8.25) or (8.39) not being the eigenvectors of the system Hamiltonian and the ability of atoms to condense into a single-particle coherent mode (8.23).

\subsection{Correlation Functions and Coherence}

As follows from the previous subsection, an equilibrium system of atoms can become totally coherent only in the thermodynamic limit at zero temperature. At finite temperatures or for a finite number of particles, a system of atoms can be only partially coherent. Since the level of coherence is directly related to the strength of correlations between atoms, this level can be characterized by the behavior of correlation functions.

The simplest correlation function is the dimensionless first-order density matrix

$$
C\left(\mathbf{r}, \mathbf{r}^{\prime}\right) \equiv \frac{<\psi^{\dagger}(\mathbf{r}) \psi\left(\mathbf{r}^{\prime}\right)>}{\sqrt{\rho(\mathbf{r}) \rho\left(\mathbf{r}^{\prime}\right)}},
$$

where $\rho(\mathbf{r}) \equiv<\hat{n}(\mathbf{r})>$. This function has the properties

$$
C^{*}\left(\mathbf{r}, \mathbf{r}^{\prime}\right)=C\left(\mathbf{r}^{\prime}, \mathbf{r}\right), \quad C(\mathbf{r}, \mathbf{r})=1 .
$$


Averaging over the coherent states $h_{N n}$ according to the definition (8.40), we have

$$
<\psi(\mathbf{r})>_{N n}=0
$$

and the density matrix writes

$$
<\psi^{\dagger}(\mathbf{r}) \psi\left(\mathbf{r}^{\prime}\right)>_{N n}=N \varphi_{n}^{*}(\mathbf{r}) \varphi_{n}\left(\mathbf{r}^{\prime}\right) .
$$

Hence, if the average in the function (8.61) is assumed as the coherent average (8.40), then

$$
\left|C\left(\mathbf{r}, \mathbf{r}^{\prime}\right)\right|=1 \quad \text { (coherence) }
$$

for all $\mathbf{r}$ and $\mathbf{r}^{\prime}$. But for the statistical average 8.46 ), in general,

$$
\left|C\left(\mathbf{r}, \mathbf{r}^{\prime}\right)\right| \leq 1
$$

with the equality occurring only for $\mathbf{r}=\mathbf{r}^{\prime}$.

An effective radius characterizing the length of strong correlations between atoms defines the coherence radius

$$
r_{c o h} \equiv \frac{\int r|C(0, \mathbf{r})| d \mathbf{r}}{\int|C(0, \mathbf{r})| d \mathbf{r}} .
$$

If this radius is less than or comparable with the mean interatomic distance,

$$
r_{\text {coh }} \leq a \quad(\text { chaos })
$$

then atoms are not correlated, which can be ascribed to chaotic behavior. When the coherence radius is much larger than the distance $a$ but much smaller than the linear size of the system,

$$
a \ll r_{\text {coh }} \ll L \quad \text { (local coherence) },
$$

then a large number of atoms are mutually correlated, although this number is much smaller than the total number of atoms in the system. And when the coherence radius is of the order of the system size,

$$
r_{c o h} \sim L \quad(\text { global coherence }),
$$

then almost all atoms in the system are correlated and practically all particles condense into a coherent mode. The correlation function (8.61) describes the property of the system, which is called first-order coherence.

The correlation function

$$
C_{2}\left(\mathbf{r}_{1}, \mathbf{r}_{2}\right) \equiv \frac{<\psi^{\dagger}\left(\mathbf{r}_{1}\right) \psi^{\dagger}\left(\mathbf{r}_{2}\right) \psi\left(\mathbf{r}_{2}\right) \psi\left(\mathbf{r}_{1}\right)>}{\rho\left(\mathbf{r}_{1}\right) \rho\left(\mathbf{r}_{2}\right)}
$$

characterizes second-order coherence. If the average here is defined as the coherent average (8.40), then

$$
C_{2}\left(\mathbf{r}_{1}, \mathbf{r}_{2}\right)=1 \quad \text { (coherence) }
$$

for any $\mathbf{r}_{1}$ and $\mathbf{r}_{2}$. The opposite case corresponds to the statistical average (8.46) under the condition that particles are not correlated, so that the average in Eq. (8.70) can be simplified using the Wick decomposition. The latter yields

$$
C_{2}\left(\mathbf{r}_{1}, \mathbf{r}_{2}\right)=1+\left|C\left(\mathbf{r}_{1}, \mathbf{r}_{2}\right)\right|^{2} \quad(\text { chaos })
$$

For this chaotic state,

$$
C_{2}\left(\mathbf{r}_{1}, \mathbf{r}_{2}\right)>1 \quad \text { (chaos) } .
$$

Combining Eqs. (8.71) and (8.72), the coinciding arguments we have

$$
C_{2}(\mathbf{r}, \mathbf{r})=\left\{\begin{array}{lc}
1, & \text { coherence } \\
2, & \text { chaos }
\end{array}\right.
$$


Similarly, the third-order coherence is described by the correlation function

$$
C_{3}\left(\mathbf{r}_{1}, \mathbf{r}_{2}, \mathbf{r}_{3}\right) \equiv \frac{<\psi^{\dagger}\left(\mathbf{r}_{1}\right) \psi^{\dagger}\left(\mathbf{r}_{2}\right) \psi^{\dagger}\left(\mathbf{r}_{3}\right) \psi\left(\mathbf{r}_{3}\right) \psi\left(\mathbf{r}_{2}\right) \psi\left(\mathbf{r}_{1}\right)>}{\rho\left(\mathbf{r}_{1}\right) \rho\left(\mathbf{r}_{2}\right) \rho\left(\mathbf{r}_{3}\right)} .
$$

In the case of the coherent average (8.40),

$$
C_{3}\left(\mathbf{r}_{1}, \mathbf{r}_{2}, \mathbf{r}_{3}\right)=1 \quad \text { (coherence) } .
$$

While for the statistical average (8.46), under the assumption that atoms are not correlated, one may employ the Wick decomposition resulting in

$$
\begin{aligned}
C_{3}\left(\mathbf{r}_{1}, \mathbf{r}_{2}, \mathbf{r}_{3}\right) & =1+\left|C\left(\mathbf{r}_{1}, \mathbf{r}_{2}\right)\right|^{2}+\left|C\left(\mathbf{r}_{2}, \mathbf{r}_{3}\right)\right|^{2}+\left|C\left(\mathbf{r}_{3}, \mathbf{r}_{1}\right)\right|^{2}+ \\
& +2 \operatorname{Re} C\left(\mathbf{r}_{1}, \mathbf{r}_{2}\right) C\left(\mathbf{r}_{2}, \mathbf{r}_{3}\right) C\left(\mathbf{r}_{3}, \mathbf{r}_{1}\right) .
\end{aligned}
$$

In the two opposite cases, we get

$$
C_{3}(\mathbf{r}, \mathbf{r}, \mathbf{r})=\left\{\begin{array}{cc}
1, & \text { coherence } \\
3 !, & \text { chaos }
\end{array}\right.
$$

The same way can be followed for characterizing higher-order coherence by means of the correlation function $C_{k}(\ldots)$ defined analogously to Eqs. (8.70) and (8.75). For the coinciding arguments, one finds

$$
C_{k}(\mathbf{r}, \mathbf{r}, \ldots, \mathbf{r})=\left\{\begin{array}{cc}
1, & \text { coherence } \\
k !, & \text { chaos }
\end{array}\right.
$$

One may notice that, if the system is coherent, this is reflected in the correlators of all orders, so that

$$
C_{k}\left(\mathbf{r}_{1}, \mathbf{r}_{2}, \ldots, \mathbf{r}_{k}\right)=1 \quad \text { (coherence) } .
$$

Thence, it is not compulsory to distinguish between different orders of coherence, but it is sufficient to use just one word "coherence". In the intermediate case, when there is neither complete coherence nor pure chaos, the properties of correlators of different orders can be different. Then one could distinguish between different orders of particle correlations.

Correlation functions can be defined not only for field operators but also for any operators. The correlator for two operators of local observables, $\hat{A}(\mathbf{r})$ and $\hat{B}(\mathbf{r})$, satisfies an important limiting property

$$
<\hat{A}\left(\mathbf{r}_{1}\right) \hat{B}\left(\mathbf{r}_{2}\right)>\simeq<\hat{A}\left(\mathbf{r}_{1}\right)><\hat{B}\left(\mathbf{r}_{2}\right)>,
$$

when $r_{12} \rightarrow \infty$, which is called the principle of correlation weakening [300, 302. This property holds only when both operators represent local observables. Since the field operators do not correspond to observable quantities, the correlator $<\psi^{\dagger}\left(\mathbf{r}_{1}\right) \psi\left(\mathbf{r}_{2}\right)>$ does not need to be factorized into the product $<\psi^{\dagger}\left(\mathbf{r}_{1}\right)><\psi\left(\mathbf{r}_{2}\right)>$, as $r_{12} \rightarrow \infty$, although it may factorize in a different form, as in Eq. (8.42). 


\section{Chapter 9}

\section{Meaning of Gross-Pitaevskii Equation}

The nonlinear Schrödinger equation (8.19) is an exact equation defining the coherent field $\varphi(\mathbf{r}, t)$ that can also be called the coherent wave function. In the nonlinear Hamiltonian (8.20), one has to specify the external potential $U$ and the interaction potential $\Phi$. For trapped atomic gases, these potentials are usually modelled as a harmonic confining potential and a contact Fermi potential, respectively [30, 31]. This concretization is given below, where we also discuss the difference between the exact equation (8.19) for the coherent wave function and some approximate equations for broken-symmetry order parameter introduced by means of the Bogolubov prescription (7.21). For concreteness, we set the normalization parameter $\kappa=N$.

\subsection{Coherent Wave Function}

The external potential $U(\mathbf{r}, t)$ in the nonlinear Hamiltonian 8.20 may, in general, consist of two terms, one describing a stationary trapping potential that is due to the trap used and another term corresponding to time-dependent perturbation superimposed on the stationary part. The trapping potential is usually modelled by a harmonic oscillator,

$$
U(\mathbf{r})=\frac{m_{0}}{2}\left(\omega_{x}^{2} x^{2}+\omega_{y}^{2} y^{2}+\omega_{z}^{2} z^{2}\right),
$$

with the frequencies defined by the confining fields of the trap.

The density of trapped gases is always small, so that the effective range of the two-body potential describing interatomic interactions is much smaller compared to the interparticle distance $a \sim \rho^{-1 / 3}$. Then the interatomic potential can be assumed to act locally and be characterized entirely by the $s$-wave scattering length $a_{s}$ [303][305]. This means that, under the condition

$$
\frac{\left|a_{s}\right|}{a} \ll 1, \quad \rho\left|a_{s}\right|^{3} \ll 1,
$$

the interaction potential can be presented in the Fermi form

$$
\Phi(\mathbf{r})=A \delta(\mathbf{r}), \quad A \equiv 4 \pi \hbar^{2} \frac{a_{s}}{m_{0}} .
$$

In typical experiments with ${ }^{87} \mathrm{Rb}$ and ${ }^{23} \mathrm{Na}$, one reaches the density $\rho \sim 10^{14} \mathrm{~cm}^{-3}$, the scattering length being $a_{s} \sim 5 \times 10^{-7} \mathrm{~cm}$, hence $a_{s} / a \sim 10^{-2}$ and $\rho a_{s}^{3} \sim 10^{-5}$. In the case of ${ }^{7} \mathrm{Li}$, one has $\rho \sim 10^{12} \mathrm{~cm}^{-3}$, with the scattering length $a_{s} \sim-10^{-7} \mathrm{~cm}$, so that $\left|a_{s}\right| / a \sim 10^{-3}$ and $\rho\left|a_{s}\right|^{3} \sim 10^{-9}$. In the Bose-Einstein condensation of atomic hydrogen [99], the density of condensed atoms is $\rho \sim 2 \times 10^{4} \mathrm{~cm}^{-3}$, while the scattering length of $\mathrm{H}$ is $a_{s} \sim 6.5 \times 10^{-9} \mathrm{~cm}$, from where $a_{s} / a \sim 10^{-4}$ and $\rho a_{s}^{3} \sim 10^{-10}$. Thus, the inequalities (9.2) always hold true.

With the trapping potential (9.1) and the interaction potential (9.3), we get the nonlinear Hamiltonian

$$
\hat{H}(\varphi)=-\frac{\hbar^{2} \nabla^{2}}{2 m_{0}}+U(\mathbf{r})+N A|\varphi|^{2} .
$$


Then the equation (8.19) for the coherent wave function takes the form

$$
i \hbar \frac{\partial \varphi}{\partial t}=[\hat{H}(\varphi)+\hat{V}] \varphi
$$

where $\hat{V}=\hat{V}(\mathbf{r}, t)$ is a time-dependent perturbation potential.

From the mathematical point of view, Eq. (9.5) is a nonlinear Schrödinger equation. This is an exact equation for the coherent wave function. Similar equations can be derived in the mean-field approximation for the order parameter associated with the condensate [31. In the latter case, one calls such equations Gross-Ginzburg-Pitaevskii equation or Gross-Pitaevskii equation, since such equations were considered by these authors [306]-310.

\subsection{Condensate Order Parameter}

The order parameter associated with the Bose-Einstein condensate is commonly defined as

$$
\psi_{0}(\mathbf{r}, t) \equiv<\psi(\mathbf{r}, t)>,
$$

that is, as the statistical average of a field operator. This definition implies that Bose-Einstein condensation is accompanied by broken gauge symmetry, which is usually done by means of the Bogolubov prescription (7.21). Substituting the Bogolubov-shifted field operator $\psi=\psi_{0}+\tilde{\psi}$ into the Heisenberg equation (8.14), and averaging the latter, one has

$$
\begin{gathered}
i \hbar \frac{\partial}{\partial t} \psi_{0}=\left(-\frac{\hbar^{2} \nabla^{2}}{2 m_{0}}+U\right) \psi_{0}+ \\
+A\left(\left|\psi_{0}\right|^{2} \psi_{0}+2<\tilde{\psi}^{\dagger} \tilde{\psi}>\psi_{0}+<\tilde{\psi} \tilde{\psi}>\psi_{0}^{*}+<\tilde{\psi}^{\dagger} \tilde{\psi} \tilde{\psi}>\right),
\end{gathered}
$$

where the dependence of functions on the space-time variables $\mathbf{r}$ and $t$, for brevity, is dropped.

Equation (9.7) for the condensate order parameter (9.6) is exact. However, it is too complicated to be useful. To simplify it, one may invoke the mean-field approximation

$$
<\tilde{\psi}^{\dagger} \tilde{\psi} \tilde{\psi}>\cong<\tilde{\psi}^{\dagger}><\tilde{\psi} \tilde{\psi}>+2<\tilde{\psi}^{\dagger} \tilde{\psi}><\tilde{\psi}>
$$

which, because of $\langle\tilde{\psi}\rangle=0$ yields

$$
<\tilde{\psi}^{\dagger} \tilde{\psi} \tilde{\psi}>=0 \text {. }
$$

Then Eq. (9.7) for the order parameter slightly simplifies becoming

$$
i \hbar \frac{\partial}{\partial t} \psi_{0}=\left(-\frac{\hbar^{2} \nabla^{2}}{2 m_{0}}+U\right) \psi_{0}+A\left(\left|\psi_{0}\right|^{2} \psi_{0}+2<\tilde{\psi}^{\dagger} \tilde{\psi}>\psi_{0}+<\tilde{\psi} \tilde{\psi}>\psi_{0}^{*}\right),
$$

which corresponds to the Hartree-Fock-Bogolubov approximation.

Sometimes one makes an ad hoc assumption that the anomalous averages $<\tilde{\psi} \tilde{\psi}>$ are much smaller than the normal ones $\left\langle\tilde{\psi}^{\dagger} \tilde{\psi}>\right.$. Then, dropping the former in Eq. (9.8), one comes to the Popov approximation

$$
i \hbar \frac{\partial}{\partial t} \psi_{0}=\left(-\frac{\hbar^{2} \nabla^{2}}{2 m_{0}}+U\right) \psi_{0}+A\left(\left|\psi_{0}\right|^{2}+2<\tilde{\psi}^{\dagger} \tilde{\psi}>\right) \psi_{0}
$$

considered first by Popov [311].

The Hartree-Fock-Bogolubov approximation is self-consistent; however, it leads to the appearance of a gap in the spectrum of elementary excitations [312]. Since in reality there is no gap, this approximation is not satisfactory. The Popov approximation yields a gapless spectrum of elementary excitations; but this approximation is not self-consistent because the anomalous averages $<\tilde{\psi} \tilde{\psi}>$ are, in general, of the order of or even much larger than the normal averages [312, 313], thus, $\langle\tilde{\psi} \tilde{\psi}\rangle$ cannot be neglected when gauge 
symmetry is broken. Moreover, the Popov approximation is unstable with respect to the formation of vortices with negative energy [312]. Therefore, the Popov approximation also cannot be accepted as satisfactory.

As discussed in subsection 7.4, the Bogolubov prescription $(7.21)$ is meaningful under the assumption of small condensate depletion, which is expressed by inequality (7.29). In the extreme case, one may assume that all particles are condensed, so that all the averages $\left\langle\tilde{\psi} \tilde{\psi}>\right.$ as well as $\left\langle\tilde{\psi}^{\dagger} \tilde{\psi}\right\rangle$ can be omitted. The procedure of neglecting all these averages, corresponding to non-condensed atoms, is often termed the Bogolubov approximation. Then Eq. (9.8) becomes

$$
i \hbar \frac{\partial}{\partial t} \psi_{0}=\left(-\frac{\hbar^{2} \nabla^{2}}{2 m_{0}}+U+A\left|\psi_{0}\right|^{2}\right) \psi_{0} .
$$

It is this approximate equation (9.10) for the order parameter (9.6) which is commonly called the GrossPitaevskii equation. If, similarly to Eq. (8.18), we change the normalization of the order parameter by means of the replacement

$$
\psi_{0}(\mathbf{r}, t)=\sqrt{N} \varphi_{0}(\mathbf{r}, t),
$$

then Eq. (9.10) takes the same form as the equations (8.19) or (9.5) for the coherent wave function. The difference is that the nonlinear Schrödinger equation (9.5) is an exact equation for the coherent wave function, while the equation (9.10) is an approximate equation for the condensate order parameter. Equation (9.5) exists irrespectively of whether gauge symmetry is broken or not, while Eq. (9.10) presupposes broken gauge symmetry. The mathematical structure of both Eqs. (9.5) and (9.10) is the same, being that of the nonlinear Schrödinger equation. What is different is their physical interpretation. However, it is admissible to accept a generalized point of view and to define the Gross-Pitaevskii equation as a nonlinear Schrödinger equation describing a system of Bose atoms, irrespectively of the interpretation of the solution to this equation.

\subsection{General Anisotropic Case}

To study the properties of the Gross-Pitaevskii equation, it is convenient to introduce some notations simplifying the following consideration. When all frequencies in the trapping potential (9.1) are different, we have the general anisotropic case. With the help of the effective frequency

$$
\omega_{0} \equiv\left(\omega_{x} \omega_{y} \omega_{z}\right)^{1 / 3},
$$

we may define the dimensionless frequencies

$$
\omega_{1} \equiv \frac{\omega_{x}}{\omega_{0}}, \quad \omega_{2} \equiv \frac{\omega_{y}}{\omega_{0}}, \quad \omega_{3} \equiv \frac{\omega_{z}}{\omega_{0}} .
$$

From these definitions, one has the property

$$
\omega_{1} \omega_{2} \omega_{3}=1 \text {. }
$$

The oscillator length

$$
l_{0} \equiv \sqrt{\frac{\hbar}{m_{0} \omega_{0}}}
$$

characterizes an effective size of the trap. This length is used for defining the dimensionless variables

$$
x_{1} \equiv \frac{r_{x}}{l_{0}}, \quad x_{2} \equiv \frac{r_{y}}{l_{0}}, \quad x_{3} \equiv \frac{r_{z}}{l_{0}}
$$

instead of the dimensional Cartesian vector $\mathbf{r}=\left\{r_{x}, r_{y}, r_{z}\right\}$. The dimensionless coupling parameter

$$
g \equiv 4 \pi \frac{a_{s}}{l_{0}} N
$$


describes the intensity of interactions between atoms in coherent state. Introducing the dimensionless nonlinear Hamiltonian

$$
\hat{H} \equiv \frac{\hat{H}(\varphi)}{\hbar \omega_{0}}
$$

and the coherent wave function

$$
\psi(\mathbf{x}) \equiv l_{0}^{3 / 2} \varphi(\mathbf{r}),
$$

we have

$$
\hat{H}=\frac{1}{2} \sum_{i=1}^{3}\left(-\frac{\partial^{2}}{\partial x_{i}^{2}}+\omega_{i}^{2} x_{i}^{2}\right)+g|\psi|^{2} .
$$

The eigenproblem 8.22 ) reduces to the form

$$
\hat{H} \psi_{n}(\mathbf{x})=E_{n} \psi_{n}(\mathbf{x}),
$$

where the energy $E_{n}$ is measured in units of $\omega_{0}$. The eigenfunction is assumed to satisfy the normalization

$$
\left(\psi_{n}, \psi_{n}\right) \equiv \int\left|\psi_{n}(\mathbf{x})\right|^{2} d \mathbf{x}=1,
$$

in which the integral is evaluated over the whole domain of $\mathbf{x}=\left\{x_{1}, x_{2}, x_{3}\right\}$.

\subsection{Cylindrically Symmetric Trap}

When the trapping potential (9.1) is cylindrically symmetric, so that the transverse radial frequencies are equal,

$$
\omega_{x}=\omega_{y}=\omega_{r},
$$

and the axial-to-radial asymmetry is described by the parameter

$$
\nu \equiv \frac{\omega_{z}}{\omega_{r}},
$$

then it is convenient to introduce the following notation. The radial oscillator length

$$
l_{r} \equiv \sqrt{\frac{\hbar}{m_{0} \omega_{r}}}
$$

serves to define the dimensionless cylindrical variables

$$
r \equiv \frac{\sqrt{r_{x}^{2}+r_{y}^{2}}}{l_{r}}, \quad z=\frac{r_{z}}{l_{r}} .
$$

Then one may define the dimensionless coherent wave function

$$
\psi(r, \varphi, z) \equiv l_{r}^{3 / 2} \varphi(\mathbf{r}),
$$

depending on the cylindrical variables $r \in[0, \infty), \varphi \in[0,2 \pi], z \in(-\infty,+\infty)$, and the Hamiltonian

$$
\hat{H} \equiv \frac{\hat{H}(\varphi(\mathbf{r}))}{\hbar \omega_{r}} .
$$

The atom-atom coupling parameter now is

$$
g \equiv 4 \pi \frac{a_{s}}{l_{r}} N .
$$


The Hamiltonian (9.26) writes

$$
\hat{H}=-\frac{1}{2} \nabla^{2}+\frac{1}{2}\left(r^{2}+\nu z^{2}\right)+g|\psi|^{2},
$$

where

$$
\nabla^{2}=\frac{\partial^{2}}{\partial r^{2}}+\frac{1}{r} \frac{\partial}{\partial r}+\frac{1}{r^{2}} \frac{\partial^{2}}{\partial \varphi^{2}}+\frac{\partial^{2}}{\partial z^{2}} .
$$

In the eigenvalue problem

$$
\hat{H} \psi_{n}=E_{n} \psi_{n},
$$

the energy $E_{n}$ is measured in units of $\omega_{r}$ and the eigenfunction $\psi_{n}=\psi_{n}(r, \varphi, z)$ is normalized by the condition

$$
\int_{0}^{\infty} r d r \int_{0}^{2 \pi} d \varphi \int_{-\infty}^{+\infty}\left|\psi_{n}(r, \varphi, z)\right|^{2} d z=1 .
$$

It is worth recalling here that the Hamiltonian (9.28) is nonlinear and, thus, non-Hermitian. Thence, the set $\left\{\psi_{n}\right\}$ of the coherent modes being the solutions of the eigenproblem (9.29) does not necessarily form a complete basis. And, in general, the set $\left\{\psi_{n}\right\}$ is not orthogonal. In particular, the eigenfunctions of non-Hermitian linear operators can form complete bi-orthonormal bases [314]- 317]. The situation with nonlinear operators is more complicated: As there are no general theorems, the completeness of the eigenfunction set is to be analyzed separately for each concrete case. For some one-dimensional problems with nonlinear Schrödinger Hamiltonians the completeness of the eigenfunction set has been proved [318, 319].

\subsection{Thomas-Fermi Ground State}

In many cases, one is interested not in the whole set of stationary states $\psi_{n}$ but solely in the ground state, $\psi_{0}$, corresponding to the minimal energy $E_{0}$. There exists a simple approximation that is very often used for describing the ground state of trapped atoms. This is the Thomas-Fermi approximation that is valid in the asymptotic limit of strong coupling parameter $g \rightarrow \infty$. Then one neglects the kinetic term as compared to the potential term containing $g$, which reduces the differential Schrödinger equation to an algebraic equation.

Thus, considering the general anisotropic case, one neglects in the Hamiltonian (9.19) the kinetic differential operator

$$
\hat{K} \equiv-\frac{1}{2} \sum_{i=1}^{3} \frac{\partial^{2}}{\partial x_{i}^{2}} .
$$

Then, Eq. (9.20) yields the wave function in the Thomas-Fermi approximation

$$
\psi_{T F}(\mathbf{x})=\frac{1}{\sqrt{2 g}}\left(r_{c}^{2}-\sum_{i=1}^{3} \omega_{i}^{2} x_{i}^{2}\right) \Theta\left(r_{c}^{2}-\sum_{i=1}^{3} \omega_{i}^{2} x_{i}^{2}\right),
$$

where $\Theta(\cdot)$ is the unit-step function and

$$
r_{c}^{2} \equiv 2 E_{T F} .
$$

The energy $E_{T F}$ is defined from the normalization $\left(\psi_{T F}, \psi_{T F}\right)=1$. Unfortunately, there is a serious defect in the wave function 9.31), since the average kinetic energy

$$
\left(\psi_{T F}, \hat{K} \psi_{T F}\right)=\sum_{i=1}^{3} \omega_{i}^{2} \int\left[1+\frac{\omega_{i}^{2} x_{i}^{2}}{\psi_{T F}^{2}(x)}\right] d \mathbf{x}
$$

logarithmically diverges.

In the cylindrically symmetric case, with the Hamiltonian (9.28), omitting the term $-\frac{1}{2} \nabla^{2}$, we find the wave function

$$
\psi_{T F}(r, z)=\left(\frac{r_{c}^{2}-r^{2}-\nu^{2} z^{2}}{2 g}\right)^{1 / 2} \Theta\left(r_{c}^{2}-r^{2}-\nu^{2} z^{2}\right),
$$


where $r_{c}$ is given by Eq. (9.32) and the energy $E_{T F}$ is to be obtained from the normalization (9.30), which yields

$$
E_{T F}=\frac{1}{2}\left(\frac{15}{4 \pi} g \nu\right)^{2 / 5}=0.536689(g \nu)^{2 / 5} .
$$

The average kinetic energy is again logarithmically divergent.

The Thomas-Fermi approximation is often used because of its simplicity. However, this approximation has several deficiencies:

First of all, as is evident from the form of the function $\psi_{T F}$ containing a unit-step function, this approximation cannot correctly describe the edge of the atomic cloud, since the Thomas-Fermi density $\left|\psi_{T F}\right|^{2}$ has a sharp boundary at $r_{c}$, while in reality the density is to be smooth [320].

Second, this approximation is not self-consistent as far as the Thomas-Fermi energy $E_{T F}$ is defined from the normalization $\left\|\psi_{T F}\right\|^{2}=1$. But if one retains the kinetic term in $\hat{H}$ then

$$
\left(\psi_{T F}, \hat{H} \psi_{T F}\right) \neq E_{T F},
$$

not even approximately. And, moreover, the average energy $\left(\psi_{T F}, \hat{H} \psi_{T F}\right)$ has no sense because of the divergence of the average kinetic energy.

Third, this approximation is applicable for describing only the ground state but does not permit the consideration of other coherent stationary states of the eigenvalue problem.

Fourth, the approximation does not make a distinction between repulsive and attractive forces, that is, between positive and negative coupling parameters $g$. However, in the case of attractive interatomic forces, there should exist a critical value $g_{c}<0$, such that for $g<g_{c}$ the system becomes unstable [30, 31, which can be manifested in the energy becoming negative or complex.

Fifth, for the time-dependent equation (9.5), the Thomas-Fermi approximation gives a solution that is unstable with respect to small perturbations [321]. 


\section{Chapter 10}

\section{Spectrum of Coherent Modes}

Coherent modes are defined by the eigenproblem (8.22). For trapped atoms, the nonlinear Hamiltonian is given by Eq. (9.4), with the trapping potential (9.1). This nonlinear eigenproblem cannot be solved exactly. The standard perturbation theory starting with a harmonic-oscillator approximation cannot be employed since the coupling parameter (9.16), or (9.27), can be very large because of large $N \gg 1$. The Thomas-Fermi approximation, as discussed in section 9.5, can give an estimate only for the ground-state energy, with $g \rightarrow \infty$. How would it be possible to find accurate approximate expressions for the whole spectrum of coherent modes and for arbitrary values of the coupling parameter? This can be achieved by means of the self-similar approximation theory whose simplest variant, called optimized perturbation theory, is outlined in the following section.

\subsection{Optimized Perturbation Theory}

Let us be interested in a function $E(g)$ of a coupling parameter $g$. We keep in mind that $E(g)=E(g, n)$ is an energy level but, for brevity, the dependence on the set of quantum numbers $n$ is not written down explicitly.

If one invokes the standard perturbation theory, valid for small coupling parameters, one gets a sequence $\left\{p_{k}(g)\right\}$ of perturbative approximations $p_{k}(g)$, with $k=0,1,2, \ldots$ implying approximation orders, so that

$$
E(g) \simeq p_{k}(g) \quad(g \rightarrow 0) .
$$

However, the perturbative sequence $\left\{p_{k}(g)\right\}$ is usually divergent for any finite value of $g$. Moreover, the coupling parameter $g$ is often not small, for which case the perturbative sequence $\left\{p_{k}(g)\right\}$ cannot in principle provide reasonable approximations.

In order to make perturbation theory meaningful, one has to change the theory so that the resulting perturbative sequence be convergent. This can be done by introducing control functions that are so called because of their role of controlling convergence. Then, instead of a divergent sequence $\left\{p_{k}(g)\right\}$, one would get a convergent sequence $\left\{E_{k}\left(g, u_{k}\right)\right\}$, whose convergence is governed by control functions $u_{k}=u_{k}(g)$. The idea of introducing control functions for making a perturbative sequence convergent was advanced first in Ref. [322]. The introduction of control functions can be done in different ways. A straightforward way is to start perturbation theory with an initial approximation containing a set of trial parameters $u$. The latter are then transformed into functions $u_{k}(g)$ such that the sequence $\left\{e_{k}(g)\right\}$ of the terms

$$
e_{k}(g) \equiv E_{k}\left(g, u_{k}(g)\right)
$$

becomes convergent. Perturbation theory reorganized, in this or that way, by introducing control functions [322] has been successfully applied to a variety of problems in quantum mechanics, statistical physics, and field theory [322]- 342]. The so reorganized perturbation theory is called by different authors differently, for instance, as optimized perturbation theory, controlled perturbation theory, modified perturbation theory, renormalized perturbation theory, oscillator-representation method, delta expansion, and so on. The method of potential envelopes [343]- 345] is also close to this approach. More references can be found in reviews [346]- 349]. 
It is only in a few simplest cases, such as zero- and one-dimensional anharmonic oscillators [350]-[352], when control functions can be chosen from the direct observation of convergence. Contrary to this, the standard situation is when perturbative terms of arbitrarily large orders are not available. Vice versa, for realistic problems, one usually is able to find just a couple of perturbative terms. Because of this, one usually defines control functions by invoking some heuristic reasons.

The foundation for the choice of control functions can be done in the frame of the self-similar approximation theory [353-[362]. These functions are to be chosen so that they provide the optimal convergence, which means that convergence is as fast as possible. Such an optimal choice of control functions results in the optimized perturbation theory. To derive the concrete equations defining control functions, it is necessary to construct a dynamical system, called the approximation cascade [358]- 362] whose trajectory is bijective to the approximation sequence $\left\{e_{k}(g)\right\}$. The limit of the latter is in one-to-one correspondence with an attractive point of the approximation cascade. Approaching the fixed point, the cascade velocity

$$
V_{k}(g)=E_{k+1}\left(g, u_{k}\right)-E_{k}\left(g, u_{k}\right)+\left(u_{k+1}-u_{k}\right) \frac{\partial}{\partial u_{k}} E_{k}\left(g, u_{k}\right)
$$

tends to zero. Hence, the closer we are to the fixed point, the smaller is the modulus of the cascade velocity (10.3). In other words, to provide the fastest convergence, control functions have to minimize the cascade velocity modulus

$$
\left|V_{k}(g)\right| \leq\left|E_{k+1}\left(g, u_{k}\right)-E_{k}\left(g, u_{k}\right)\right|+\left|\left(u_{k+1}-u_{k}\right) \frac{\partial}{\partial u_{k}} E_{k}\left(g, u_{k}\right)\right| .
$$

From here, two variants of the fixed-point conditions can be written down: either the minimal-difference condition

$$
E_{k+1}\left(g, u_{k}\right)-E_{k}\left(g, u_{k}\right)=0
$$

or the minimal-sensitivity condition

$$
\left(u_{k+1}-u_{k}\right) \frac{\partial}{\partial u_{k}} E_{k}\left(g, u_{k}\right)=0 .
$$

The latter, since in general $u_{k+1} \neq u_{k}$, reduces to the variational condition

$$
\frac{\partial}{\partial u_{k}} E_{k}\left(g, u_{k}\right)=0 \text {. }
$$

Both conditions, (10.5) and (10.7), are widely used in various applications. When it happens that equations (10.5) or (10.7) have no solutions, these equations can be generalized to the condition

$$
\min _{u}\left|E_{k+1}(g, u)-E_{k}(g, u)\right|
$$

or, respectively, to the condition

$$
\min _{n}\left|\frac{\partial}{\partial u} E_{k}(g, u)\right|
$$

The accuracy of the optimized approximants $(10.2)$, as compared to the known value $E(g)$, is characterized by the percentage errors

$$
\varepsilon_{k}(g) \equiv \frac{e_{k}(g)-E(g)}{|E(g)|} \times 100 \% .
$$

Let us emphasize the difference between the optimized perturbation theory and the variational procedure based on the minimization of the internal-energy functional: First, the latter has sense solely for the ground state while the former is valid for the whole spectrum of the eigenproblem. Second, the latter implies the case of zero temperature, while the former is independent of temperature. Third, the minimization of the internal energy yields an optimal value for the energy itself, but the described method provides optimal approximants for the spectrum. 


\subsection{Isotropic Ground State}

In general, the eigenproblem (9.20) involves all three space variables. The situation can be simplified when the confining potential (9.1) is spherically symmetric, so that $\omega_{x}=\omega_{y}=\omega_{z}$, and if we are interested only in the ground state. In this case, the ground-state wavefunction

$$
\psi_{0}(\mathbf{x})=\frac{1}{\sqrt{4 \pi} r} \chi(r)
$$

depends solely on $r \equiv|\mathbf{x}|$. Then the eigenproblem (9.20) can be reduced to the effective equation

$$
\begin{gathered}
\hat{H}_{r} \chi=E \chi, \\
\hat{H}_{r} \equiv \frac{1}{2}\left(-\frac{d^{2}}{d r^{2}}+r^{2}\right)+\frac{g}{4 \pi r^{2}} \chi^{2},
\end{gathered}
$$

containing only the radial variable $r$. The radial wave function $\chi(r)$, because of the relation (10.11), has to satisfy the condition $\chi(0)=0$.

The Rayleigh-Schrödinger perturbation theory can be started with the harmonic Hamiltonian

$$
\hat{H}_{r}^{(0)} \equiv-\frac{1}{2} \frac{d^{2}}{d r^{2}}+\frac{u^{2}}{2} r^{2}
$$

including a parameter $u$ that will later generate control functions $u_{k}(g)$. The ground-state eigenfunction of the Hamiltonian (10.13) is

$$
\chi^{(0)}(r)=2\left(\frac{u^{3}}{\pi}\right)^{1 / 4} r \exp \left(-\frac{1}{2} u r^{2}\right) .
$$

The first-order approximation for the ground-state energy writes

$$
E^{(1)}(g, u)=\left(\chi^{(0)}, \hat{H}_{r} \chi^{(0)}\right) .
$$

It is convenient to introduce the notation

$$
s \equiv \frac{2 g}{(2 \pi)^{3 / 2}},
$$

which characterizes an effective interaction strength. Then, Eq. (10.14) yields

$$
E^{(1)}(g, u)=\frac{3}{4}\left(u+\frac{1}{u}\right)+\frac{s}{2} u^{3 / 2} .
$$

Using the fixed-point condition

$$
\frac{\partial}{\partial u} E^{(1)}(g, u)=0
$$

we get the equation

$$
s u^{5 / 2}+u^{2}-1=0
$$

defining the control function $u=u(s)$. In general, the control function equation (10.18) is to be solved numerically. But for weak and strong interaction strengths, we may derive the following asymptotic expansions: for the weak-coupling limit, $s \rightarrow 0$,

$$
u(s) \simeq 1-\frac{1}{2} s+\frac{1}{2} s^{2}-\frac{39}{64} s^{3}+\frac{105}{128} s^{4},
$$

and for the strong-coupling limit, $s \rightarrow \infty$,

$$
u(s) \simeq s^{-2 / 5}-\frac{2}{5} s^{-6 / 5}+\frac{1}{5} s^{-2}-\frac{12}{125} s^{-14 / 5} .
$$


Substituting the control function $u(s)$ into Eq. (10.16) gives the first-order optimized approximant

$$
E(s) \equiv E_{1}(g(s), u(s)) .
$$

Its behavior in the weak-coupling limit is

$$
E(s) \simeq \frac{3}{2}+\frac{1}{2} s-\frac{3}{16} s^{2}+\frac{9}{64} s^{3}-\frac{35}{256} s^{4},
$$

where $s \rightarrow 0$; and in the strong-coupling limit, we have

$$
E(s) \simeq \frac{5}{4} s^{2 / 5}+\frac{3}{4} s^{-2 / 5}-\frac{3}{20} s^{-6 / 5}+\frac{1}{20} s^{-2}-\frac{9}{500} s^{-14 / 5},
$$

as $s \rightarrow \infty$. Following the optimized perturbation theory described in Sec. 10.1, one can obtain optimized approximants of arbitrary orders. However, we limit ourselves here to the first-order approximants.

For atoms with negative scattering length, as in the case of ${ }^{7} \mathrm{Li}$ or ${ }^{85} \mathrm{Rb}$, the coupling parameter (9.16) is negative, hence the parameter (10.15) is negative, too. If $s<0$, the control function equation (10.18) has real solutions only in the interval $s_{c}<s<0$, where

$$
s_{c}=-\frac{4}{5^{5 / 4}}=-0.534992 .
$$

The ground-state energy (10.19) is real in the same interval of $s \in\left(s_{c}, 0\right)$ and becomes complex for $s<s_{c}$. This corresponds to the interval $g_{c}<g<0$, with the critical coupling parameter

$$
g_{c}=\frac{(2 \pi)^{3 / 2}}{2} s_{c}=-4.212960 .
$$

The fact that there is a critical value for the coupling parameter (9.16) can be reformulated as the existence of a critical number of particles

$$
N_{c}=\frac{l_{0} g_{c}}{4 \pi a_{s}}
$$

that can form a coherent state. Thus, for the parameters of the experiments [0, 110] with ${ }^{7} \mathrm{Li}$, we get $N_{c} \sim 10^{3}$.

When the ground-state energy becomes complex, this means that the system is unstable. The lifetime of such a system can be estimated as

$$
\tau(g) \equiv \frac{1}{\omega_{0}|\operatorname{Im} e(g)|},
$$

where $e(g)=E(s(g))$. In the limit $g \rightarrow-\infty$, we have 139] the asymptotic expansions

$$
\begin{gathered}
\operatorname{Re} e(g) \simeq 0.169198 g^{2 / 5}+0.529102 g^{-2 / 5}+1.443899 g^{-6 / 5}+31.006277 g^{-2}, \\
\operatorname{Im} e(g) \simeq-0.520739 g^{2 / 5}+1.628409 g^{-2 / 5}+1.049054 g^{-6 / 5} .
\end{gathered}
$$

Therefore, in this limit the lifetime 10.24 ) can be estimated as

$$
\tau(g) \simeq \frac{1.920}{\omega_{0}|g|^{2 / 5}} \quad(g \rightarrow-\infty) .
$$

Note that if one defines the critical coupling $g_{c}$ not from the direct solution of the eigenproblem (10.12) but from the minimization of the internal-energy functional [31], then the resulting critical coupling is about twice as large as the value (10.22). 


\subsection{Anisotropic Excited States}

When the confining potential is not isotropic or when we are interested not solely in the ground state but also in the spectrum of excited coherent modes, we have to deal with the eigenproblem (9.20). Then, for developing optimized perturbation theory, we may start with the Hamiltonian

$$
\hat{H}_{0}=\frac{1}{2} \sum_{i=1}^{3}\left(-\frac{\partial^{2}}{\partial x_{i}^{2}}+u_{i}^{2} x_{i}^{2}\right),
$$

containing three trial parameters, $u_{1}, u_{2}$, and $u_{3}$, generating control functions. The Hamiltonian (10.26) possesses the eigenvalues

$$
E_{n}^{(0)}=\sum_{i=1}^{3} u_{i}\left(n_{i}+\frac{1}{2}\right)
$$

with the eigenfunctions

$$
\begin{gathered}
\psi_{n}^{(0)}(\mathbf{x})=\prod_{i=1}^{3} \psi_{n_{i}}\left(x_{i}\right) \\
\psi_{n_{i}}\left(x_{i}\right)=\frac{\left(u_{i} / \pi\right)^{1 / 4}}{\sqrt{2^{n_{i}} n_{i} !}} H_{n_{i}}\left(\sqrt{u_{i}} x_{i}\right) \exp \left(-\frac{1}{2} u_{i} x_{i}^{2}\right),
\end{gathered}
$$

where $n \equiv\left\{n_{1}, n_{2}, n_{3}\right\} ; n_{i}=0,1,2, \ldots$; and $H_{n_{i}}(\cdot)$ is a Hermite polynomial. Perturbation theory is accomplished [139] with respect to the perturbation

$$
\Delta H \equiv \hat{H}-\hat{H}_{0}=\frac{1}{2} \sum_{i=1}^{3}\left(\omega_{i}^{2}-u_{i}^{2}\right) x_{i}^{2}+g|\psi|^{2} .
$$

In the first order, one has

$$
E_{n}^{(1)}(g, u)=E_{n}^{(0)}+\left(\psi_{n}, \Delta H \psi_{n}\right),
$$

where $u \equiv\left\{u_{1}, u_{2}, u_{3}\right\}$. Introducing the effective interaction strength

$$
s \equiv 2 g \prod_{i=1}^{3} I_{n_{i}}
$$

in which

$$
I_{n_{i}} \equiv \frac{2}{\pi\left(2^{n_{i}} n_{i} !\right)^{2}} \int_{0}^{\infty} H_{n_{i}}^{4}(x) e^{-2 x^{2}} d x
$$

where the property $\left|H_{n}(-x)\right|=\left|H_{n}(x)\right|$ of the Hermite polynomials is used, one obtains for the energy levels (10.29)

$$
E_{n}^{(1)}(g, u)=\frac{1}{2} \sum_{i=1}^{3} u_{i}\left(n_{i}+\frac{1}{2}\right)\left(1+\frac{\omega_{i}^{2}}{u_{i}^{2}}\right)+\frac{1}{2} \sqrt{u_{1} u_{2} u_{3}} s .
$$

Control functions, playing the role of effective oscillation frequencies, are defined by the fixed-point condition

$$
\frac{\partial}{\partial u_{i}} E_{n}^{(1)}(g, u)=0
$$

which results in three equations

$$
\left(n_{i}+\frac{1}{2}\right)\left(u_{i}^{2}-\omega_{i}^{2}\right)+\frac{u_{i}}{2} \sqrt{u_{1} u_{2} u_{3}} s=0
$$


defining $u_{i}=u_{i}(s)$, where $i=1,2,3$ and, for short, the quantum multi-index $n$ is dropped. The first-order optimized approximant is given by

$$
E(s) \equiv E_{n}^{(1)}(g(s), u(s))
$$

where again, for brevity, the index $n$ is omitted in the left-hand side.

The control-function equations (10.33) yield

$$
u_{i}(s) \simeq \omega_{i}-\frac{s}{2\left(2 n_{i}+1\right)}+\frac{\omega_{i}}{4}\left[\frac{\sum_{j=1}^{3}\left(2 n_{j}+1\right) \omega_{j}-\left(2 n_{i}+1\right) \omega_{i}}{2 \prod_{j=1}^{3}\left(2 n_{j}+1\right)}+\frac{1}{\left(2 n_{i}+1\right)^{2} \omega_{i}^{2}}\right] s^{2}
$$

in the weak-coupling limit $s \rightarrow 0$, and

$$
u_{i}(s) \simeq \frac{\left(2 n_{i}+1\right) \omega_{i}^{2}}{\prod_{j=1}^{3}\left(2 n_{j}+1\right)^{1 / 5}} s^{-2 / 5}
$$

in the strong-coupling limit $s \rightarrow \infty$. For the energy levels (10.34), we find

$$
E(s) \simeq \sum_{i=1}^{3} \omega_{i}\left(n_{i}+\frac{1}{2}\right)+\frac{s}{2}-\frac{1}{16} \sum_{i=1}^{3} \frac{s^{2}}{\left(2 n_{i}+1\right) \omega_{i}}
$$

in the weak-coupling limit $s \rightarrow 0$, and

$$
E(s) \simeq \frac{5}{4} \prod_{j=1}^{3}\left(2 n_{j}+1\right)^{1 / 5} s^{2 / 5}
$$

in the strong-coupling limit $s \rightarrow \infty$.

For the ground state, when $n_{i}=0$, the coupling strength (10.30) reduces to Eq. 10.15). Then for the control functions, we have

$$
u_{i}(s) \simeq \omega_{i}-\frac{s}{2}+\frac{\omega_{i}}{4}\left[\frac{1}{2}\left(\sum_{j=1}^{3} \omega_{j}-\omega_{i}\right)+\frac{1}{\omega_{i}^{2}}\right] s^{2},
$$

if $s \rightarrow 0$, and

$$
u_{i}(s) \simeq \omega_{i}^{2} s^{-2 / 5},
$$

when $s \rightarrow \infty$. In these two limits, the ground-state energy is

$$
E_{0}(s) \simeq \frac{1}{2}\left(\omega_{1}+\omega_{2}+\omega_{3}\right)+\frac{s}{2}-\frac{1}{16}\left(\frac{1}{\omega_{1}}+\frac{1}{\omega_{2}}+\frac{1}{\omega_{3}}\right) s^{2},
$$

for $s \rightarrow 0$, and

$$
E_{0}(s) \simeq \frac{5}{4} s^{2 / 5}=0.547538 g^{2 / 5}
$$

as $s \rightarrow \infty$. In the isotropic case, we return to Eqs. (10.20) and (10.21).

The arrangement of the energy levels in the weak-coupling and strong-coupling limits is, in general, different. This can be illustrated by considering several first energy levels $e_{n}(g) \equiv E_{n}(s(g))$. For example,

$$
\begin{array}{ll}
e_{000}(g) \simeq \frac{3}{2}+0.063 g & e_{100}(g) \simeq \frac{5}{2}+0.048 g, \\
e_{110}(g) \simeq \frac{7}{2}+0.036 g, & e_{200}(g) \simeq \frac{7}{2}+0.041 g,
\end{array}
$$

in the weak-coupling limit $g \rightarrow 0$, and

$$
e_{000}(g) \simeq 0.547 g^{2 / 5}, \quad e_{100}(g) \simeq 0.608 g^{2 / 5},
$$




$$
e_{200}(g) \simeq 0.632 g^{2 / 5}, \quad e_{110}(g) \simeq 0.675 g^{2 / 5}
$$

for $g \rightarrow \infty$. As is seen from here,

$$
e_{110}(g)<e_{200}(g) \quad(g \rightarrow 0)
$$

but

$$
e_{110}(g)>e_{200}(g) \quad(g \rightarrow \infty) .
$$

This effect is called level crossing 139].

In the case of negative scattering lengths, when $g<0$, the situation is analogous to that studied in Sec. 10.2. For each given energy level, labelled by $n$, there exists a critical value of the coupling parameter $g=g_{c}$ when the corresponding energy becomes complex. Then the lifetime of an energy level, with the complex energy $e_{n}(g)$, can be estimated as

$$
\tau_{n}(g) \equiv \frac{1}{\omega_{0}\left|\operatorname{Im} e_{n}(g)\right|} .
$$

The spatial shape of the cloud of trapped atoms is characterized by the aspect ratio

$$
R_{i} \equiv\left(\frac{<x_{i}^{2}>_{n}}{<x_{3}^{2}>_{n}}\right)^{1 / 2} \quad(i=1,2)
$$

in which $<x_{i}^{2}>_{n}$ implies $\left(\psi_{n}, x_{i}^{2} \psi_{n}\right)$. For the function $\psi_{n}^{(0)}(\mathbf{x})$, this gives

$$
R_{i}=\left[\frac{\left(2 n_{i}+1\right) u_{3}}{\left(2 n_{3}+1\right) u_{i}}\right]^{1 / 2}
$$

In the weak-coupling limit,

$$
R_{i} \simeq\left[\frac{\left(2 n_{i}+1\right) \omega_{3}}{\left(2 n_{3}+1\right) \omega_{i}}\right]^{1 / 2}\left\{1+\left[\frac{1}{\left(2 n_{i}+1\right) \omega_{i}}-\frac{1}{\left(2 n_{3}+1\right) \omega_{3}}\right] \frac{g}{2(2 \pi)^{3 / 2}}\right\},
$$

as $g \rightarrow 0$, while in the strong-coupling limit,

$$
R_{i} \simeq \frac{\omega_{3}}{\omega_{i}} \quad(g \rightarrow \infty)
$$

This tells us that the shape of different coherent modes essentially depends on the quantum numbers $n_{i}$, if $g \rightarrow 0$, but for large coupling parameters, the shape of different modes is practically the same, tending to that of Eq. (10.45).

\subsection{Cylindric Trapping Potential}

For a cylindrically symmetric trap, it is convenient to use the notations introduced in Sec. 9.4. Cylindrical traps are often employed in experiments, therefore we shall pay more attention to this case.

To solve the eigenproblem (9.29), we may again invoke the optimized perturbation theory of Sec. 10.1, starting with the initial Hamiltonian

$$
\hat{H}_{0}=-\frac{1}{2} \nabla^{2}+\frac{1}{2}\left(u^{2} r^{2}+v^{2} z^{2}\right)
$$

containing two control parameters, $u$ and $v$. The eigenvalues of the operator (10.46) are

$$
E_{n m k}^{(0)}=(2 n+|m|+1) u+\left(k+\frac{1}{2}\right) v,
$$

with the quantum numbers

$$
n=0,1,2, \ldots ; \quad m=0, \pm 1, \pm 2, \ldots ; \quad k=0,1,2, \ldots
$$


The related eigenfunctions are

$$
\begin{gathered}
\psi_{n m k}^{(0)}(r, \varphi, z)=\left[\frac{2 n ! u^{|m|+1}}{(n+|m|) !}\right]^{1 / 2} r^{|m|} \exp \left(-\frac{1}{2} u r^{2}\right) L_{n}^{|m|}\left(u r^{2}\right) \times \\
\times \frac{e^{i m \varphi}}{\sqrt{2 \pi}} \frac{(v / \pi)^{1 / 4}}{\sqrt{2^{k} k !}} \exp \left(-\frac{1}{2} v z^{2}\right) H_{k}(\sqrt{v} z),
\end{gathered}
$$

where $L_{n}^{m}(\cdot)$ is a Laguerre polynomial and $H_{k}(\cdot)$ is a Hermite polynomial.

In the first order, we have

$$
E_{n m k}^{(1)}(g, u, v)=\left(\psi_{n m k}^{(0)}, \hat{H} \psi_{n m k}^{(0)}\right) .
$$

To write down this integral explicitly, it is convenient to use the notation

$$
I_{n m k} \equiv \frac{1}{u \sqrt{v}} \int\left|\psi_{n m k}^{(0)}(\mathbf{r})\right|^{4} d \mathbf{r},
$$

in which $\mathbf{r}=\{r, \varphi, z\}$ is the dimensionless space variable in cylindrical coordinates. Then we get

$$
I_{n m k}=\frac{2}{\pi^{2}}\left[\frac{n !}{(n+|m|) ! 2^{k} k !}\right]^{2} \int_{0}^{\infty} x^{2|m|} e^{-2 x}\left[L_{n}^{|m|}(x)\right]^{4} d x \int_{0}^{\infty} e^{-2 t^{2}} H_{k}^{4}(t) d t .
$$

It is also convenient to introduce the notation

$$
p \equiv 2 n+|m|+1, \quad q \equiv 2 k+1 .
$$

In this way, the energy levels (10.48) can be written as

$$
E^{(1)}(g, u, v)=\frac{p}{2}\left(u+\frac{1}{u}\right)+\frac{q}{4}\left(v+\frac{\nu^{2}}{v}\right)+I_{n m k} u \sqrt{v} g,
$$

where, for simplicity, the quantum indices $n, m$, and $k$ in the left-hand side are dropped.

The fixed-point conditions are

$$
\frac{\partial}{\partial u} E^{(1)}(g, u, v)=0, \quad \frac{\partial}{\partial v} E^{(1)}(g, u, v)=0 .
$$

These yield the control-function equations

$$
p\left(1-\frac{1}{u^{2}}\right)+\frac{s}{p \nu} \sqrt{\frac{v}{q}}=0, \quad q\left(1-\frac{\nu^{2}}{v^{2}}\right)+\frac{s}{p \nu \sqrt{\nu q}}=0,
$$

in which the effective interaction strength

$$
s \equiv 2 p \sqrt{q} I_{n m k} \nu g
$$

is introduced. Substituting the control functions $u=u(s)$ and $v=v(s)$, defined by Eqs. (10.52), into Eq. (10.50), we obtain the optimized approximant

$$
E(s) \equiv E^{(1)}(g(s), u(s), v(s)),
$$

where $g(s)$ is given by the relation (10.53).

Similarly to the previous sections, it is instructive to analyze the weak-coupling and strong-coupling limits in detail. In the weak-coupling limit $s \rightarrow 0$, Eqs. (10.52) give the radial control function

$$
u(s) \simeq 1-\frac{1}{2 p^{2}(q \nu)^{1 / 2}} s+\frac{p+3 q \nu}{8 p^{4}(q \nu)^{2}} s^{2}-\frac{3 p^{2}+16 p q \nu+20(q \nu)^{2}}{64 p^{6}(q \nu)^{7 / 2}} s^{3}
$$


and, respectively, the axial control function

$$
v(s) \simeq \nu-\frac{\nu}{2 p(q \nu)^{3 / 2}} s+\frac{\nu(p+q \nu)}{4 p^{3}(q \nu)^{3}} s^{2}-\nu \frac{7 p^{2}+20 p q \nu+12(q \nu)^{2}}{64 p^{5}(q \nu)^{9 / 2}} s^{3} .
$$

In the strong-coupling limit $s \rightarrow \infty$, the radial control function is

$$
u(s) \simeq u_{1} s^{-2 / 5}+u_{2} s^{-6 / 5}+u_{3} s^{-2}+u_{4} s^{-14 / 5}+u_{5} s^{-18 / 5},
$$

where

$$
\begin{gathered}
u_{1}=p, \quad \frac{5}{p} u_{2}=-3 p^{2}+(q \nu)^{2}, \quad \frac{5}{p} u_{3}=3 p^{4}-p^{2}(q \nu)^{2}-(q \nu)^{4}, \\
\frac{125}{p} u_{4}=-88 p^{6}+33 p^{4}(q \nu)^{2}+4 p^{2}(q \nu)^{4}+39(q \nu)^{6} \\
\frac{625}{p} u_{5}=561 p^{8}-238 p^{6}(q \nu)^{2}-21 p^{4}(q \nu)^{4}+88 p^{2}(q \nu)^{6}-364(q \nu)^{8} .
\end{gathered}
$$

And for the axial control function, we get

$$
v(s) \simeq v_{1} s^{-2 / 5}+v_{2} s^{-6 / 5}+v_{3} s^{-2}+v_{4} s^{-14 / 5}+v_{5} s^{-18 / 5},
$$

where $s \rightarrow \infty$ and

$$
\begin{gathered}
v_{1}=q \nu^{2}, \quad \frac{5}{2 q \nu^{2}} v_{2}=p^{2}-2(q \nu)^{2}, \quad \frac{5}{q \nu^{2}} v_{3}=-p^{4}-4 p^{2}(q \nu)^{2}+6(q \nu)^{4}, \\
\frac{125}{2 q \nu^{2}} v_{4}=11 p^{6}+4 p^{4}(q \nu)^{2}+117 p^{2}(q \nu)^{4}-138(q \nu)^{6}, \\
\frac{625}{q \nu^{2}} v_{5}=-119 p^{8}-28 p^{6}(q \nu)^{2}+264 p^{4}(q \nu)^{4}-2912 p^{2}(q \nu)^{6}+2821(q \nu)^{8} .
\end{gathered}
$$

The weak-coupling limit for the energy (10.54) becomes

$$
E(s) \simeq a_{0}+a_{1} s+a_{2} s^{2}+a_{3} s^{3},
$$

as $s \rightarrow 0$, where

$$
a_{0}=p+\frac{q \nu}{2}, \quad a_{1}=\frac{1}{2 p(q \nu)^{1 / 2}}, \quad a_{2}=-\frac{p+2 q \nu}{16 p^{3}(q \nu)^{2}}, \quad a_{3}=\frac{(p+2 q \nu)^{2}}{64 p^{5}(q \nu)^{7 / 2}} .
$$

And in the strong-coupling limit, we find

$$
E(s) \simeq b_{0} s^{2 / 5}+b_{1} s^{-2 / 5}+b_{2} s^{-6 / 5}+b_{3} s^{-2}+b_{4} s^{-14 / 5}+b_{5} s^{-18 / 5},
$$

as $s \rightarrow \infty$, with

$$
\begin{gathered}
b_{0}=\frac{5}{4}, \quad 4 b_{1}=2 p^{2}+(q \nu)^{2}, \quad 20 b_{2}=-3 p^{4}+2 p^{2}(q \nu)^{2}-2(q \nu)^{4}, \\
20 b_{3}=2 p^{6}-p^{4}(q \nu)^{2}-2 p^{2}(q \nu)^{4}+2(q \nu)^{6}, \\
500 b_{4}=-44 p^{8}+22 p^{6}(q \nu)^{2}+2 p^{4}(q \nu)^{4}+78 p^{2}(q \nu)^{6}-69(q \nu)^{8}, \\
12500 b_{5}=1122 p^{10}-595 p^{8}(q \nu)^{2}-70 p^{6}(q \nu)^{4}+440 p^{4}(q \nu)^{6}-3640 p^{2}(q \nu)^{8}+2821(q \nu)^{10} .
\end{gathered}
$$

The derived expressions (10.59) and (10.60) are valid for any combination of quantum numbers. 


\subsection{Cloud Shape and Lifetime}

The shape of an atomic cloud in a cylindrical trap can be characterized by the mean-square radial and axial lengths, respectively,

$$
r_{0} \equiv\left(<r^{2}>_{n m k}\right)^{1 / 2}, \quad z_{0} \equiv\left(<z^{2}>_{n m k}\right)^{1 / 2},
$$

where $<\cdot>_{n m k}$ is a quantum-mechanical average over the wave function $\psi_{n m k}$. Taking these averages with respect to the function $\psi_{n m k}^{(0)}$, we have

$$
r_{0}=\sqrt{\frac{p}{u}}, \quad z_{0}=\sqrt{\frac{q}{2 v}} .
$$

In the weak-coupling limit $s \rightarrow 0$, the radial mean-square deviation is

$$
r_{0} \simeq \sqrt{p}\left(1+\frac{1}{4 p^{2}(q \nu)^{1 / 2}} s-\frac{2 p+3 q \nu}{32 p^{4}(q \nu)^{2}} s^{2}+\frac{3 p^{2}+10 p q \nu+7(q \nu)^{2}}{128 p^{6}(q \nu)^{7 / 2}} s^{3}\right),
$$

and the axial mean-square length is

$$
z_{0} \simeq \sqrt{\frac{q}{2 \nu}}\left(1+\frac{1}{4 p(q \nu)^{3 / 2}} s-\frac{p+4 q \nu}{32 p^{3}(q \nu)^{3}} s^{2}+\frac{2 p+3 q \nu}{32 p^{5}(q \nu)^{7 / 2}} s^{3}\right) .
$$

In the strong-coupling limit $s \rightarrow \infty$, for the radial and axial averages (10.62) we find

$$
r_{0} \simeq s^{1 / 5}+\frac{3 p^{2}-(q \nu)^{2}}{10} s^{-3 / 5}-\frac{33 p^{4}-2 p^{2}(q \nu)^{2}-23(q \nu)^{4}}{200} s^{-7 / 5}
$$

and, respectively,

$$
z_{0} \simeq \frac{1}{\sqrt{2} \nu}\left(s^{1 / 5}-\frac{p^{2}-2(q \nu)^{2}}{5} s^{-3 / 5}+\frac{4 p^{4}+4 p^{2}(q \nu)^{2}-9(q \nu)^{4}}{25} s^{-7 / 5}\right) .
$$

For the aspect ratio

$$
R_{r} \equiv\left(\frac{<r^{2}>_{n m k}}{<z^{2}>_{n m k}}\right)^{1 / 2}=\frac{r_{0}}{z_{0}}
$$

we have

$$
R_{r}=\sqrt{\frac{2 p v}{q u}} .
$$

This gives in the weak-coupling limit

$$
R_{r}(s) \simeq \sqrt{\frac{2 p \nu}{q}}\left(1+\alpha_{1} s+\alpha_{2} s^{2}+\alpha_{3} s^{3}+\alpha_{4} s^{4}\right),
$$

where $s \rightarrow 0$, and

$$
\begin{gathered}
4 p^{2}(q \nu)^{3 / 2} \alpha_{1}=-p+q \nu, \quad 32 p^{4}(q \nu)^{3} \alpha_{2}=3 p^{2}-3(q \nu)^{2}, \\
128 p^{6}(q \nu)^{9 / 2} \alpha_{3}=-4 p^{3}-8 p^{2} q \nu+5 p(q \nu)^{2}+7(q \nu)^{3}, \\
2048 p^{8}(q \nu)^{6} \alpha_{4}=15 p^{4}+112 p^{3} q \nu+70 p^{2}(q \nu)^{2}-120 p(q \nu)^{3}-77(q \nu)^{4} .
\end{gathered}
$$

In the strong-coupling limit, the aspect ratio (10.68) is

$$
R_{r}(s) \simeq \sqrt{2} \nu\left(1+\beta_{1} s^{-4 / 5}+\beta_{2} s^{-8 / 5}+\beta_{3} s^{-12 / 5}+\beta_{4} s^{-16 / 5}\right),
$$

where $s \rightarrow \infty$, and

$$
2 \beta_{1}=p^{2}-(q \nu)^{2}, \quad \frac{40}{9} \beta_{2}=-p^{4}-2 p^{2}(q \nu)^{2}+3(q \nu)^{4},
$$




$$
\begin{gathered}
400 \beta_{3}=77 p^{6}+5 p^{4}(q \nu)^{2}+391 p^{2}(q \nu)^{4}-473(q \nu)^{6}, \\
\frac{16000}{13} \beta_{4}=-253 p^{8}-4 p^{6}(q \nu)^{2}+266 p^{4}(q \nu)^{4}-2900 p^{2}(q \nu)^{6}+2891(q \nu)^{8} .
\end{gathered}
$$

These expansions confirm that the dependence of the aspect ratio on quantum numbers diminishes in the strong-coupling limit, so that

$$
\lim _{s \rightarrow \infty} R_{r}(s)=\sqrt{2} \nu
$$

in agreement with Eq. (10.45).

Considering the stationary properties of coherent modes, we should not forget that in real traps atoms cannot be confined infinitely long. This is because the trapping of neutral atoms requires their special spin polarization which can be lost during atomic collisions [363. One usually considers binary and triple depolarizing collisions [364, 365]. The corresponding loss rate of atoms in a coherent mode $\psi_{n m k}$ can be written as

$$
\Gamma_{2} \equiv \lambda_{2} N^{2} \gamma_{n m k}, \quad \Gamma_{3} \equiv \lambda_{3} N^{3} \delta_{n m k},
$$

where $\lambda_{2}$ is a two-body dipolar loss rate coefficient, $\lambda_{3}$ is a three-body recombination loss rate coefficient, and

$$
\gamma_{n m k} \equiv \int\left|\psi_{n m k}(\mathbf{r})\right|^{4} d \mathbf{r}, \quad \delta_{n m k} \equiv \int\left|\psi_{n m k}(\mathbf{r})\right|^{6} d \mathbf{r} .
$$

For alkali atoms [364, 365], the two-body loss rate coefficient is $\lambda_{2} \sim 10^{-16}-10^{-15} \mathrm{~cm}^{3} / \mathrm{s}$ and the three-body one is $\lambda_{3} \sim 10^{-30}-10^{-28} \mathrm{~cm}^{6} / \mathrm{s}$, the lifetime of atoms in a trap is on the order of $1-100 \mathrm{~s}$.

The integrals (10.72), with $\psi_{n m k}^{(0)}$, take the form

$$
\gamma_{n m k}=u \sqrt{v} I_{n m k}, \quad \delta_{n m k}=u^{2} v J_{n m k},
$$

where $I_{n m k}$ is the same integral as in Sec. 10.4 and

$$
J_{n m k} \equiv \frac{2}{\pi^{7 / 2}}\left[\frac{n !}{(n+|m|) ! 2^{k} k !}\right]^{3} \int_{0}^{\infty} x^{3|m|} e^{-3 x}\left[L_{n}^{|m|}(x)\right]^{6} d x \int_{0}^{\infty} e^{-3 t^{2}} H_{k}^{6}(t) d t .
$$

In the weak-coupling limit, when $s \rightarrow 0$, the quantities (10.73) are

$$
\gamma_{n m k}(s) \simeq \sqrt{\nu} I_{n m k}, \quad \delta_{n m k}(s) \simeq \nu J_{n m k},
$$

and in the strong-coupling limit, we have

$$
\gamma_{n m k}(s) \simeq p \sqrt{q} \nu I_{n m k} s^{-3 / 5}, \quad \delta_{n m k} \simeq p^{2} q \nu^{2} J_{n m k} s^{-6 / 5},
$$

as $s \rightarrow \infty$. Since $s \sim N$, the quantities (10.75) decrease with increasing number of particles. However, the loss rates (10.71) increase with $N$ according to the laws $\Gamma_{2} \sim N^{7 / 5}$ and $\Gamma_{3} \sim N^{9 / 5}$.

To compare the loss rates of excited coherent modes with those of the ground state, we may analyze the reduced loss rates

$$
\bar{\gamma}_{n m k}(s) \equiv \frac{\gamma_{n m k}(s)}{\gamma_{000}(s)}, \quad \bar{\delta}_{n m k}(s) \equiv \frac{\delta_{n m k}(s)}{\delta_{000}(s)} .
$$

The latter possess the properties

$$
\bar{\gamma}_{n m k}(0) \leq \bar{\gamma}_{n m k}(s) \leq \bar{\gamma}_{n m k}(\infty), \quad \bar{\delta}_{n m k}(0) \leq \bar{\delta}_{n m k}(s) \leq \bar{\delta}_{n m k}(\infty)
$$

In order to get a feeling in what range the reduced loss rates vary, we may consider several first states. For this purpose, we need to calculate the corresponding integrals $I_{n m k}$ and $J_{n m k}$. For instance, for the ground state,

$$
I_{000}=\frac{1}{(2 \pi)^{3 / 2}}=0.063494, \quad J_{000}=\frac{1}{\left(3 \pi^{2}\right)^{3 / 2}}=0.006207 .
$$


And for several first excited states,

$$
\begin{array}{ll}
I_{100}=0.031747, & J_{100}=0.002222, \\
I_{010}=0.031747, & J_{010}=0.001379, \\
I_{001}=0.047620, & J_{001}=0.003448 .
\end{array}
$$

In the case of $n=k=0$, but arbitrary $m$,

$$
I_{0 m 0}=\frac{(2|m|) !}{(2 \pi)^{3 / 2}\left(2^{|m|}|m| !\right)^{2}} \quad J_{0 m 0}=\frac{(3|m|) !}{\left(3 \pi^{2}\right)^{3 / 2}\left(3^{|m|}|m|\right)^{3}} .
$$

In this way, we find

$$
\begin{aligned}
& 0.5 \leq \bar{\gamma}_{100}(s) \leq 1.18, \quad 0.36 \leq \bar{\delta}_{100}(s) \leq 1.98, \\
& 0.5 \leq \bar{\gamma}_{010}(s) \leq 1, \quad 0.22 \leq \bar{\delta}_{010}(s) \leq 0.88, \\
& 0.75 \leq \bar{\gamma}_{001}(s) \leq 1.11 \quad 0.56 \leq \bar{\delta}_{001}(s) \leq 1.22 .
\end{aligned}
$$

Thus, the loss rates of excited states are close to those of the ground state. 


\section{Chapter 11}

\section{Weak-to-Strong Coupling Crossover}

When considering the properties of trapped atoms at arbitrary coupling parameters, one usually needs to invoke computer calculations. Analytical expressions can be available only in the weak-coupling and strong-coupling limits. Nevertheless, there is a method permitting one to reconstruct an analytical formula, valid for the whole region of coupling parameters, for a function whose asymptotic expansions in the weak-coupling and strongcoupling limits are known. Here we briefly delineate this method and then apply it for describing several properties of trapped atoms.

\subsection{Self-Similar Crossover Approximants}

Assume that we are interested in the behavior of a function $f(s)$ of the coupling parameter $s$. Let this function be defined by a complicated equation that can be solved only numerically. But we can find the asymptotic expansion

$$
f(s) \simeq a_{0}+a_{1} s+a_{2} s^{2}+\ldots \quad(s \rightarrow 0)
$$

in the weak-coupling limit. And we can often analytically derive the asymptotic expansion

$$
f(s) \simeq b_{0} s^{\beta_{0}}+b_{1} s^{\beta_{1}}+b_{2} s^{\beta_{2}}+\ldots \quad(s \rightarrow \infty)
$$

in the strong-coupling limit, where the powers $\beta_{j}$ are arranged in the decreasing order, $\beta_{j}>\beta_{j+1}$.

Introducing into series (11.1) control functions by means of an algebraic transformation [366]-368] and using the self-similar approximation theory [353]- 362], we obtain [369, 370] the self-similar root approximant

$$
f_{k}^{*}(s)=a_{0}\left(\ldots\left\{\left[\left(1+A_{1} s\right)^{n_{1}}+A_{2} s^{2}\right]^{n_{2}}+A_{3} s^{3}\right\}^{n_{3}}+\ldots+A_{k} s^{k}\right)^{n_{k}},
$$

where $k$ is the order of the approximation taken. The coefficients $A_{j}$ and powers $n_{j}$ are to be defined by considering the strong-coupling limit of the approximant (11.3) and equating it to the strong-coupling expansion (11.2). This way can be called the left-to-right crossover.

In general, it could be possible to go the opposite way, from the right to the left. That is, we could construct a nested-root approximant starting from the strong-coupling asymptotic form (11.2) and then define the corresponding coefficients and powers by equating the approximant expansion in the weak-coupling limit to the asymptotic expansion (11.1). However, the right-to-left crossover results in approximants that usually are less accurate than the left-to-right crossover formulas. This is connected to the fact that the weak-coupling expansions have, as a rule, zero radius of convergence, while the strong-coupling ones have a finite radius of convergence. The accuracy of the left-to-right crossover approximants is usually better than that of the rightto-left ones because of the larger region of applicability of the strong-coupling expansion (11.2) as compared to the region of validity of the weak-coupling expansion (11.1). In fact, the latter can be valid for $s \ll 1$, hence its region of validity is inside the interval $[0,1)$. In contrast, the strong-coupling form, derived for $s \gg 1$, has 
the region of applicability inside the interval $(1, \infty)$. Therefore the self-similar crossover approximant has to be fitted to the asymptotic expansion that possesses the larger region of validity.

When considering the strong-coupling limit $s \rightarrow \infty$ for the approximant (11.3), we need to know the relation between the powers $n_{j}$ and the numbers $j=1,2, \ldots$. Among all possible relations, we have to choose that one which is the most general, imposing no restrictions on the powers $\beta_{j}$. It is possible to show that the condition

$$
j n_{j}<j+1 \quad(j=1,2, \ldots, k-1)
$$

provides a general way of expanding the form (11.3), valid for any $k=1,2, \ldots$ and arbitrary $\beta_{j}$.

Under the criterion (11.4), and rewriting the approximant (11.3) in the form

$$
\begin{aligned}
f_{k}^{*}(s)= & a_{0}\left(A_{k} s^{k}\right)^{n_{k}}\left(1+\frac{A_{k-1}^{n_{k-1}}}{A_{k}} x^{k-(k-1) n_{k-1}}\left\{1+\frac{A_{k-2}^{n_{k-2}}}{A_{k-1}} x^{k-1-(k-2) n_{k-2}}(1+\ldots\right.\right. \\
& \left.\left.\left.\ldots+\frac{A_{2}^{n_{2}}}{A_{3}} x^{3-2 n_{2}}\left[1+\frac{A_{1}^{n_{1}}}{A_{2}} x^{2-n_{1}}\left(1+\frac{x}{A_{1}}\right)^{n_{1}}\right]^{n_{2}}\right)^{n_{3}} \ldots\right\}^{n_{k-1}}\right)^{n_{k}},
\end{aligned}
$$

where $x \equiv s^{-1}$, it is easy to expand the latter in powers of $x$. Comparing the resulting expansion with the strong-coupling limit (11.2), we obtain

$$
\begin{gathered}
k n_{k}=\beta_{0}, \\
(k-j) n_{k-j}=\beta_{j}-\beta_{j-1}+k-j+1,
\end{gathered}
$$

with $1 \leq j \leq k-1$. The values of $n_{j}$, defined by Eqs. (11.5), are in compliance with the criterion (11.4) because of the inequality $\beta_{j}-\beta_{j-1}<0$.

The first-order self-similar approximant (11.3) is

$$
f_{1}^{*}(s)=a_{0}(1+A s)^{n_{1}},
$$

where

$$
A^{n_{1}}=\frac{b_{0}}{a_{0}}, \quad n_{1}=\beta_{0} .
$$

The second-order approximant (11.3) takes the form

$$
f_{2}^{*}(s)=a_{0}\left[\left(1+A_{1} s\right)^{n_{1}}+A_{2} s^{2}\right]^{n_{2}},
$$

in which

$$
\begin{aligned}
A_{1}^{n_{1} n_{2}} & =\frac{b_{0}}{a_{0}}\left(\frac{b_{1}}{n_{2} b_{0}}\right)^{n_{2}}, \quad A_{2}^{n_{2}}=\frac{b_{0}}{a_{0}}, \\
n_{1} & =\beta_{1}-\beta_{0}+2, \quad 2 n_{2}=\beta_{0} .
\end{aligned}
$$

In the third order, we find

$$
f_{3}^{*}(s)=a_{0}\left\{\left[\left(1+B_{1} s\right)^{n_{1}}+B_{2} s^{2}\right]^{n_{2}}+B_{3} s^{3}\right\}^{n_{3}},
$$

where

$$
\begin{gathered}
B_{1}^{n_{1} n_{2} n_{3}}=\frac{b_{0}}{a_{0}}\left(\frac{b_{1}}{n_{3} b_{0}}\right)^{n_{3}}\left(\frac{b_{2}}{n_{2} b_{1}}-\frac{n_{3}-1}{2 n_{2} n_{3}} \frac{b_{1}}{b_{0}}\right)^{n_{2} n_{3}}, \\
B_{2}^{n_{2} n_{3}}=\frac{b_{0}}{a_{0}}\left(\frac{b_{1}}{n_{3} b_{0}}\right)^{n_{3}}, \quad B_{3}^{n_{3}}=\frac{b_{0}}{a_{0}}, \\
n_{1}=\beta_{2}-\beta_{1}+2, \quad 2 n_{2}=\beta_{1}-\beta_{0}+3, \quad 3 n_{3}=\beta_{0} .
\end{gathered}
$$

The method of constructing self-similar crossover formulas is also applicable to asymptotic expansions more general than Eq. (11.1), for instance, to series

$$
f(s)=a_{0}+a_{1} s^{\alpha_{1}}+a_{2} s^{\alpha_{2}}+\ldots \quad(s \rightarrow 0),
$$


in which $\alpha_{j}$ are arbitrary positive powers arranged in the increasing order as

$$
0<\alpha_{j}<\alpha_{j+1}
$$

Then, instead of Eq. (11.3), we obtain the self-similar approximant

$$
f_{k}^{*}(s)=a_{0}\left(\ldots\left\{\left[\left(1+A_{1} s^{\alpha_{1}}\right)^{n_{1}}+A_{2} s^{\alpha_{2}}\right]^{n_{2}}+A_{3} s^{\alpha_{3}}\right\}^{n_{3}}+\ldots+A_{k} s^{\alpha_{k}}\right)^{n_{k}} .
$$

The criterion (11.4) transforms to the inequality

$$
\alpha_{j} n_{j}<\alpha_{j+1} .
$$

And, in the place of Eqs. (11.5), we find

$$
\begin{gathered}
\alpha_{k} n_{k}=\beta_{0}, \\
\alpha_{j} n_{j}=\alpha_{j+1}+\beta_{k-j}-\beta_{k-j-1},
\end{gathered}
$$

with $j=1,2, \ldots, k-1$.

The described method makes it possible to construct analytical interpolative formulas for the whole range of the coupling parameter. The method can also be used for interpolating any functions of other variables, provided the corresponding asymptotic expansions are available.

\subsection{One-Dimensional Confined System}

To illustrate the method presented in the previous section, let us consider a model case of a one-dimensional system of trapped atoms 369. This means that in the eigenproblem $\hat{H} \psi=E \psi$, we consider the nonlinear Hamiltonian

$$
\hat{H}=-\frac{1}{2} \frac{d^{2}}{d x^{2}}+\frac{1}{2} x^{2}+g|\psi|^{2},
$$

in which $x \in(-\infty,+\infty)$.

In order to derive the weak-coupling and strong-coupling asymptotic expansions, we may resort to the optimized perturbation theory of Sec. 10.1. To this end, we start with the trial Hamiltonian

$$
\hat{H}_{0}=-\frac{1}{2} \frac{d^{2}}{d x^{2}}+\frac{u^{2}}{2} x^{2},
$$

containing a control parameter $u$, possessing the eigenvalue

$$
E_{n}^{(0)}=\left(n+\frac{1}{2}\right) u
$$

and having the eigenfunction

$$
\psi_{n}^{(0)}(x)=\frac{(u / \pi)^{1 / 4}}{\sqrt{2^{n} n !}} H_{n}(\sqrt{u} x) \exp \left(-\frac{1}{2} u x^{2}\right)
$$

where $n=0,1,2, \ldots$.

The first-order approximation gives

$$
E_{n}^{(1)}(g, u)=\frac{u}{2}\left(n+\frac{1}{2}\right)\left(1+\frac{1}{u^{2}}\right)+\sqrt{u} I_{n} g
$$

with the notation

$$
I_{n} \equiv \frac{2}{\pi\left(2^{n} n !\right)^{2}} \int_{0}^{\infty} H_{n}^{4}(x) e^{-2 x^{2}} d x .
$$


The variational condition for Eq. (11.13) yields the equation

$$
u^{2}+\alpha u^{3 / 2}-1=0
$$

for the control function $u=u(\alpha)$, where

$$
\alpha \equiv \frac{2 I_{n} g}{2 n+1}
$$

For the optimized approximant

$$
E(\alpha) \equiv E_{n}^{(1)}(g(\alpha), u(\alpha))
$$

we have

$$
E(\alpha)=\frac{1}{2}\left(n+\frac{1}{2}\right)\left(\frac{3}{u}-u\right) .
$$

Expression (11.17), together with the control-function equation (11.14), results in the weak-coupling expansion

$$
E(\alpha) \simeq\left(n+\frac{1}{2}\right)\left(1+\alpha-\frac{1}{8} \alpha^{2}+\frac{1}{32} \alpha^{3}-\frac{1}{128} \alpha^{4}+\frac{3}{2048} \alpha^{5}\right)
$$

for $\alpha \rightarrow 0$, and in the strong-coupling expansion

$$
E(\alpha) \simeq\left(n+\frac{1}{2}\right)\left(\frac{3}{2} \alpha^{2 / 3}+\frac{1}{2} \alpha^{-2 / 3}-\frac{1}{6} \alpha^{-2}+\frac{7}{54} \alpha^{-10 / 3}\right),
$$

as $\alpha \rightarrow \infty$.

Following Sec. 11.1, we find the self-similar crossover approximants. In the first order, this gives

$$
E_{1}^{*}(\alpha)=\left(n+\frac{1}{2}\right)(1+A \alpha)^{2 / 3}
$$

with $A=\frac{3}{4} \sqrt{6}$. In the second order, we find

$$
E_{2}^{*}(\alpha)=\left(n+\frac{1}{2}\right)\left[\left(1+A_{1} \alpha\right)^{2 / 3}+A_{2} \alpha^{2}\right]^{1 / 3}
$$

where

$$
A_{1}=\frac{81}{32} \sqrt{6}, \quad A_{2}=\frac{27}{8} .
$$

Formulas (11.20) and (11.21) interpolate between the weak-coupling expansion (11.18) and the strong-coupling limit (11.19).

A similar interpolation procedure can be applied for constructing an analytical expression for the groundstate wave function [369. The latter, at small space variable, has an expansion

$$
\psi(x) \simeq c_{0}+c_{2} x^{2}+c_{4} x^{4} \quad(x \rightarrow 0),
$$

and in the opposite limit

$$
\psi(x) \simeq C \exp \left(-\frac{1}{2} x^{2}\right) \quad(x \rightarrow \infty) .
$$

The interpolating formula, sewing the limits $(11.22)$ and $(11.23)$, can be constructed invoking the self-similar exponential approximants [371, which results in

$$
\psi_{*}(x)=C \exp \left\{-\frac{1}{2} x^{2}+a x^{2} \exp \left(-b x^{2}\right)\right\} .
$$

Here, the coefficients $a$ and $b$ are to be defined by expanding the function 11.24 in powers of $x \rightarrow 0$ and substituting this expansion into the eigenproblem $\hat{H} \psi_{*}=E \psi_{*}$, which yields

$$
a=\frac{1}{2}+g C^{2}-E, \quad b=\frac{2 E-4 a E-1}{12 a} .
$$


The normalization constant $C$ and energy $E$ are defined by the equations

$$
\left(\psi_{*}, \psi_{*}\right)=1, \quad E_{*}=\left(\psi_{*}, \hat{H} \psi_{*}\right)
$$

where $E \equiv E_{*}$.

The self-similar approximant (11.24) for the wave function is different from the ground-state wave function

$$
\psi_{0}^{(0)}(x)=\left(\frac{u}{\pi}\right)^{1 / 4} \exp \left(-\frac{u}{2} x^{2}\right),
$$

with the control function $u(\alpha)$ defined by the variational equation $(11.14)$, with $\alpha=\sqrt{(2 / \pi)} g$. And in the Thomas-Fermi approximation, we have the wave function

$$
\psi_{T F}(x)=\left(\frac{x_{c}^{2}-x^{2}}{2 g}\right)^{1 / 2} \Theta\left(x_{c}-|x|\right)
$$

with the energy

$$
E_{T F}(g)=\frac{x_{c}^{2}}{2}=\frac{1}{2}\left(\frac{3}{2} g\right)^{2 / 3}
$$

To compare these different approximations, we consider the properties of the ground-state energy $E(g)$, as a function of the coupling parameter $g$, presented by the optimized approximant $E_{\text {opt }}(g) \equiv E(\alpha(g))$ given by Eq. (11.17), by the crossover approximant $E_{2}^{*}(g)$ from Eq. (11.21), by the energy $E_{*}(g)$ defined in Eq. (11.25), and by the Thomas-Fermi energy $E_{T F}(g)$ in Eq. (11.28). We also compare the shape of the density

$$
n(x) \equiv|\psi(x)|^{2},
$$

defined for the corresponding functions (11.24), (11.26), and (11.27). The accuracy of the approximations can be characterized by substituting the wave functions into the Schrödinger equation and calculating the residual $R(x)$ and dispersion $\sigma^{2}$, given by the equations

$$
R(x) \equiv(\hat{H}-E) \psi(x), \quad \sigma^{2} \equiv \int_{-\infty}^{+\infty}|R(x)|^{2} d x .
$$

Figure 26 shows the energies $E_{*}(g), E_{2}^{*}(g)$, and $E_{T F}(g)$. The first two energies are almost indistinguishable from each other. The optimized approximant $E_{\text {opt }}(g)$ is not shown since it practically coincides with $E_{*}(g)$. The Thomas-Fermi energy $E_{T F}(g)$ has an incorrect weak-coupling limit and becomes a reasonable approximation for $g \geq 7$.

The density $(11.29)$ for the self-similar wave function (11.24), Gaussian function (11.26) and Thomas-Fermi wave function (11.27) is presented in Fig. 27 for different coupling parameters. As is seen, the self-similar function (11.24) has the correct behavior in both weak-coupling as well as strong-coupling limits, while the Gaussian function (11.26) does not present a good approximation in the strong-coupling limit and the ThomasFermi function (11.27) is not correct in the weak-coupling limit. In addition, the latter function is always incorrect at the boundary of an atomic cloud.

The accuracy of the corresponding approximate solutions to the nonlinear Schrödinger equation is well illustrated by the residual $R(x)$, which is shown in Fig. 28. We also calculated the dispersion $\sigma^{2}$ for different coupling parameters within the region $0 \leq g \leq 100$. The maximal, with respect to $g$, dispersion for the self-similar function (11.24) is of order one, for the Gaussian function (11.26) it is about 400, and for the Thomas-Fermi function (11.27) it is divergent. This clearly proves that the self-similar function (11.24) is the most accurate solution to the nonlinear Schrödinger equation for small as well as for large coupling parameters.

\subsection{Spherically Symmetric Trap}

Similarly to the model one-dimensional case considered above, we can construct self-similar crossover approximants for the realistic three-dimensional situation. We shall illustrate this for a spherically symmetric trap. To this end, let us consider the isotropic ground state studied in Sec. 10.2. 
Using the weak-coupling and strong-coupling expansions, (10.20) and respectively (10.21), for the groundstate energy (10.19), we construct the crossover formulas of first order,

$$
E_{1}^{*}(s)=\frac{3}{2}(1+A s)^{2 / 5},
$$

where $A=0.633938$; of second order,

$$
E_{2}^{*}(s)=\frac{3}{2}\left[\left(1+A_{1} s\right)^{6 / 5}+A_{2} s^{2}\right]^{1 / 5},
$$

with $A_{1}=1.168636$ and $A_{2}=0.401878$; of third order,

$$
E_{3}^{*}(s)=\frac{3}{2}\left\{\left[\left(1+B_{1} s\right)^{6 / 5}+B_{2} s^{2}\right]^{11 / 10}+B_{3} s^{3}\right\}^{2 / 15},
$$

where $B_{1}=1.633061, B_{2}=1.132289$, and $B_{3}=0.254766$; of fourth order,

$$
E_{4}^{*}(s)=\left(\left\{\left[\left(1+C_{1} s\right)^{6 / 5}+C_{2} s^{2}\right]^{11 / 10}+C_{3} s^{3}\right\}^{16 / 15}+C_{4} s^{4}\right)^{1 / 10},
$$

with $C_{1}=2.066398, C_{2}=2.111737, C_{3}=0.970940, C_{4}=0.161506 ;$ and of fifth order,

$$
E_{5}^{*}(s)=\frac{3}{2}\left[\left(\left\{\left[\left(1+D_{1} s\right)^{6 / 5}+D_{2} s^{2}\right]^{11 / 10}+D_{3} s^{3}\right\}^{16 / 15}+D_{4} s^{4}\right)^{21 / 20}+D_{5} s^{5}\right]^{2 / 25},
$$

where the coefficients are $D_{1}=2.479006, D_{2}=3.311734, D_{3}=2.278301, D_{4}=0.777603$, and $D_{5}=0.102385$. The variable $s$ is defined in Eq. (10.15). Note that $A>A_{2}>B_{3}>C_{4}>D_{5}$, which suggests that the accuracy of $E_{k}^{*}(s)$ should increase with increasing $k$. The accuracy of the crossover approximants $E_{k}^{*}(s)$ can be characterized by the percentage errors

$$
\varepsilon_{k}^{*}(s) \equiv \frac{E_{k}^{*}(s)-E(s)}{|E(s)|} \times 100 \%,
$$

calculated with respect to the optimized approximant (10.19). Even more instructive are the maximal errors

$$
\varepsilon_{k}^{*} \equiv \max _{s} \varepsilon_{k}^{*}(s) \quad(0 \leq s \leq \infty)
$$

For the latter, we find

$$
\varepsilon_{1}^{*}=3.7 \%, \quad \varepsilon_{2}^{*}=1.4 \%, \quad \varepsilon_{3}^{*}=0.8 \%, \quad \varepsilon_{4}^{*}=0.6 \%, \quad \varepsilon_{5}^{*}=0.4 \%,
$$

which demonstrates good numerical convergence of the crossover approximants for the ground-state energy.

A crossover approximant for the radial wave function satisfying Eq. (11.12) can also be constructed by sewing the small-radius limit

$$
\chi(r) \simeq c_{1} r+c_{3} r^{3}+c_{5} r^{5} \quad(r \rightarrow 0)
$$

and the large-distance asymptotic form

$$
\chi(r) \simeq C r \exp \left(-\frac{1}{2} r^{2}\right) \quad(r \rightarrow \infty) .
$$

The self-similar crossover formula is

$$
\chi_{*}(r)=C r \exp \left\{-\frac{1}{2} r^{2}+a r^{2} \exp \left(-b r^{2}\right)\right\} .
$$


Here the coefficients $a$ and $b$ are to be found by expanding Eq. (11.38) in powers of $r$ and substituting this expansion into the equation (10.12). Equating the coefficients at the like powers of $r$, we get

$$
a=\frac{1}{2}+\frac{g C^{2}-4 \pi E}{12 \pi}, \quad b=\frac{2(1-2 a) E-2(1-2 a)^{2}-1}{20 a} .
$$

The normalization coefficient $C$ and energy $E$ are defined by the equations

$$
E_{*}=\int_{0}^{\infty} \chi_{*}(r) \hat{H}_{r} \chi(r) d r, \quad \int_{0}^{\infty} \chi_{*}^{2}(r) d r=1
$$

where $E_{*} \equiv E$.

In this way, we obtain several representations for the ground-state energy: The self-similar approximant $E_{*}$, given in Eq. (11.39), the crossover approximants $E_{k}^{*}$ in Eqs. (11.31) to (11.35), the optimized approximant $E(s)$ defined by Eq. (10.19), and the Thomas-Fermi approximation $E_{T F}$ from Eq. (9.34), with $\nu=1$. Similarly to the previous section, we may analyze the behavior of these approximations as functions of the coupling parameter $g$. The analysis shows that the self-similar approximant $E_{*}(g)$, defined in Eq. (11.39), gives the best approximation, valid for the whole range of the parameter $g \in[0, \infty)$, correctly interpolating the weak-coupling expansion and the Thomas-Fermi limit. The latter gives a good approximation only for $g>300$, essentially

deviating from the weak-coupling form, as is seen in Fig. 29. The percentage errors $\varepsilon_{k}^{*}(s)$ of different crossover approximants $E_{k}^{*}(s)$ are presented in Figs. 30 and 31. The maximal error occurs around $g \sim 1$.

The spatial density

$$
n(r) \equiv \frac{\chi^{2}(r)}{r^{2}}
$$

is expressed through the corresponding radial function, for which one can take either the self-similar form $\chi_{*}(r)$ in Eq. (11.38), or the Gaussian approximation $\chi^{(0)}(r)$ from Sec. 10, or the Thomas-Fermi wave function

$$
\chi_{T F}(r)=r \sqrt{\frac{2 \pi}{g}\left(r_{c}^{2}-r^{2}\right)} \Theta\left(r_{c}^{2}-r^{2}\right), \quad r_{c}^{2}=2 E_{T F}, \quad E_{T F}=\frac{1}{2}\left(\frac{15}{4 \pi} g\right)^{2 / 5} .
$$

The accuracy of the considered approximate solutions can again be characterized by the residual

$$
R(r) \equiv\left(\hat{H}_{r}-E\right) \chi(r)
$$

and the dispersion

$$
\sigma^{2} \equiv \int_{0}^{\infty}|R(r)|^{2} d r
$$

The analysis here is similar to the previous section, and again the self-similar form (11.38) turns out to be the best approximation, valid for all coupling parameters. Thus, the residual as well as the dispersion diverge for the Thomas-Fermi approximation at any $g$. The accuracy of the Gaussian variational approximation is good for small $g$ but decreases with increasing $g$. For instance, the dispersion $\sigma^{2}$ for the Gaussian approximation monotonically rises with $g$, being e.g. at $g=2513$, equal to $\sigma^{2}=13.2$. At the same time, the dispersion for the self-similar approximant (11.38) reaches the maximum of $\sigma^{2}=4.1$ at $g=2411$ and then again diminishes to $\sigma^{2}=1.1$ at $g=2513$.

\subsection{Traps of Cylindrical Shape}

Self-similar crossover approximants can also be constructed for cylindrical traps, using the expansions of Sec. 10.4. Recall that these expansions, being done in terms of the variable

$$
s \equiv 2 p \sqrt{q} I_{n m k} \nu g \quad(p \equiv 2 n+|m|+1, q \equiv 2 k+1),
$$

are valid for arbitrary excited coherent modes labelled by the quantum numbers $n, m$, and $k$. 
Being based on the weak-coupling, (10.59), and strong-coupling, (10.60), expansions for the energy levels, and employing the technique of Sec. 11.1, we obtain the crossover approximants $E_{k}^{*}(s)$. Thus, in the first order, we have

$$
E_{1}^{*}(s)=a_{0}(1+A s)^{2 / 5}
$$

where

$$
a_{0}=p+\frac{q \nu}{2}, \quad A=\frac{1.746928}{a_{0}^{5 / 2}} .
$$

The second order yields

$$
E_{2}^{*}(s)=a_{0}\left[\left(1+A_{1} s\right)^{6 / 5}+A_{2} s^{2}\right]^{1 / 5},
$$

with the same $a_{0}$ and with

$$
A_{1}=\frac{2.533913}{a_{0}^{25 / 6}}\left[2 p^{2}+(q \nu)^{2}\right]^{5 / 6}, \quad A_{2}=\frac{3.051758}{a_{0}^{5}} .
$$

In the third order, we get

$$
E_{3}^{*}(s)=a_{0}\left\{\left[\left(1+B_{1} s\right)^{6 / 5}+B_{2} s^{2}\right]^{11 / 10}+B_{3} s^{3}\right\}^{2 / 15}
$$

where

$$
\begin{gathered}
B_{1}=\frac{1.405455}{a_{0}^{125 / 22}\left[2 p^{2}+(q \nu)^{2}\right]^{5 / 66}}\left[8 p^{4}+12 p^{2}(q \nu)^{2}+(q \nu)^{4}\right]^{5 / 6}, \\
B_{2}=\frac{6.619620}{a_{0}^{75 / 11}}\left[2 p^{2}+(q \nu)^{2}\right]^{10 / 11} \quad B_{3}=\frac{5.331202}{a_{0}^{15 / 2}} .
\end{gathered}
$$

Similarly, we find $E_{4}^{*}(s)$ and $E_{5}^{*}(s)$, although we do not write them down explicitly.

To check the accuracy of the crossover approximants $E_{k}^{*}(s)$, we calculate the percentage errors $\varepsilon_{k}^{*}(s)$ comparing $E_{k}^{*}(s)$ with the optimized approximant (10.54). We have calculated the maximal errors $\varepsilon_{k}^{*} \equiv \max _{s} \varepsilon_{k}^{*}(s)$ for the anisotropy parameter $\nu$, defined in Eq. (9.22), in the range $0.1 \leq \nu \leq 100$ for the ground state and for ten first excited states. For example, for the ground state, with $n=m=k=0$, and for $\nu=1$, we find

$$
\varepsilon_{1}^{*}=3.7 \%, \quad \varepsilon_{2}^{*}=1.4 \%, \quad \varepsilon_{3}^{*}=0.8 \%, \quad \varepsilon_{4}^{*}=0.6 \%, \quad \varepsilon_{5}^{*}=0.4 \%,
$$

which demonstrates good convergence. In the case of a cigar-shape trap, with $\nu=0.1$, we obtain

$$
\varepsilon_{1}^{*}=8 \%, \quad \varepsilon_{2}^{*}=3.5 \%, \quad \varepsilon_{3}^{*}=2 \%, \quad \varepsilon_{4}^{*}=1.2 \%, \quad \varepsilon_{5}^{*}=0.8 \% .
$$

For a disk-shape trap, with $\nu=10$, we have

$$
\varepsilon_{1}^{*}=12.5 \%, \quad \varepsilon_{2}^{*}=3 \% \quad \varepsilon_{3}^{*}=2.8 \%, \quad \varepsilon_{4}^{*}=-1.8 \% .
$$

The same good convergence occurs for excited states with different quantum numbers and for various anisotropy parameters. The standard situation is such that $\varepsilon_{1}^{*} \approx 4-12 \%, \varepsilon_{2}^{*} \approx 2-5 \%$, and already the third-order approximant has $\varepsilon_{3}^{*} \sim 1 \%$.

To illustrate in more detail the accuracy of the crossover approximants $E_{k}^{*}$ as functions of the coupling parameter $g$, we show in Fig. 32 the percentage errors of $E_{1}^{*}, E_{2}^{*}$, and $E_{3}^{*}$ for several levels and different anisotropy parameters. The errors are calculated with respect to the optimized approximant (10.54) whose ground-state behavior is presented in Fig. 33, where the Thomas-Fermi energy is also given for comparison.

In the same way, we may construct the crossover approximants for the aspect ratio (10.68), being based on the asymptotic expansions (10.69) and (10.70). Here, it is more convenient to deal with the quantity

$$
\bar{R}_{r}(s) \equiv \frac{R_{r}(s)}{\sqrt{2} \nu}-1,
$$


for which Eqs. (10.69) and (10.70) transform to the expansions

$$
\bar{R}_{r}(s) \simeq\left(\sqrt{\frac{p}{q \nu}}-1\right)+\sqrt{\frac{p}{q \nu}}\left(\alpha_{1} s+\alpha_{2} s^{2}+\ldots\right)
$$

in the weak-coupling limit $s \rightarrow 0$, and to

$$
\bar{R}_{r}(s) \simeq \beta_{1} s^{-4 / 5}+\beta_{2} s^{-8 / 5}+\ldots
$$

in the strong-coupling limit $s \rightarrow \infty$. Constructing the self-similar approximant $\bar{R}_{r}^{*}(s)$, we then return to the aspect ratio

$$
R_{r}^{*}(s)=\sqrt{2} \nu\left[1+\bar{R}_{r}^{*}(s)\right] .
$$

We have compared the accuracy of the crossover approximants, corresponding to Eq. (11.45), with the value (10.68) for the anisotropy parameter $\nu$ in the range $0<\nu \leq 100$, and for the first ten energy levels. The results are similar to those obtained for the energy levels themselves.

\subsection{Strong-Coupling and Thermodynamic Limits}

The atom-atom coupling (9.27) is proportional to the number of atoms $N$, which suggests that the strongcoupling limit $g \rightarrow \infty$ has to be related to the thermodynamic limit $N \rightarrow \infty$. The averages of observable quantities $\hat{A}$ should behave in the thermodynamic limit so that

$$
\lim _{N \rightarrow \infty}\left|\frac{1}{N}<\hat{A}>\right|<\infty .
$$

Let us check this property for the coherent averages of the Hamiltonian (8.13). For the latter, the coherent average (8.54) coincides with the pure coherent average (8.31), that is with (8.38). For the normalization (8.51), we have

$$
<H>_{N n}=\hbar \omega_{r} N\left(S_{n}+\frac{1}{2} g \gamma_{n}\right),
$$

where $n$ implies the whole set of quantum numbers $n, m$, and $k$; the mean single-particle energy is

$$
S_{n} \equiv \int \psi_{n}^{*}(\mathbf{r})\left[-\frac{1}{2} \nabla^{2}+\frac{1}{2}\left(r^{2}+\nu^{2} z^{2}\right)\right] \psi_{n}(\mathbf{r}) d \mathbf{r},
$$

with dimensionless $\mathbf{r}$ measured in units of $l_{r}$, defined in Eq. (9.23); and $\gamma_{n}$ being the same as $\gamma_{n m k}$ in Eq. (10.72). From the eigenproblem (9.29) it follows that

$$
E_{n} \equiv\left(\psi_{n}, \hat{H} \psi_{n}\right)=S_{n}+g \gamma_{n}
$$

Hence, the average energy (11.47) of a coherent state can also be presented in two other forms as

$$
<H>_{N n}=\frac{1}{2} \hbar \omega_{r} N\left(E_{n}+S_{n}\right), \quad<H>_{N n}=\hbar \omega_{r} N\left(E_{n}-\frac{1}{2} g \gamma_{n}\right) .
$$

Note that one should not confuse here $H$, which is the system Hamiltonian (8.13), with $\hat{H}$, which is the Schrödinger Hamiltonian (9.28).

Consider the strong-coupling limit $g \rightarrow \infty$ for the coherent average energy (11.47) or (11.49). According to the notation (10.53), this corresponds to $s \rightarrow \infty$. Then, Eqs. (10.60) and (10.75) yield

$$
E_{n}(s) \simeq \frac{5}{4} s^{2 / 5}, \quad \gamma_{n}(s) \simeq \frac{1}{2 g} s^{2 / 5} \quad(s \rightarrow \infty) .
$$


In this limit, the coherent single-particle energy (11.48) is

$$
S_{n}(s)=E_{n}(s)-g \gamma_{n} \simeq \frac{3}{4} s^{2 / 5} .
$$

Therefore,

$$
<H>_{N n} \simeq \hbar \omega_{r} N s^{2 / 5} \quad(s \rightarrow \infty) .
$$

From the definition of the coupling (9.27), we have

$$
g=4 \pi \sqrt{\frac{\omega_{r}}{\varepsilon}} N, \quad \varepsilon \equiv \frac{\hbar}{m_{0} a_{s}^{2}} .
$$

And the relation (10.53) between $g$ and $s$ gives

$$
s=C_{n}^{5 / 2}\left(\frac{\omega_{r}}{\varepsilon}\right)^{1 / 2} N, \quad C_{n} \equiv\left(8 \pi \sqrt{q} I_{n} \nu\right)^{2 / 5} .
$$

Then, the average energy (11.51) becomes

$$
<H>_{N n} \simeq C_{n} \hbar \varepsilon\left(\frac{\omega_{r}}{\varepsilon}\right)^{6 / 5} N^{7 / 5},
$$

as $N \rightarrow \infty$.

Note that in the strong-coupling limit, when $s \rightarrow \infty$, the average kinetic energy

$$
K_{n} \equiv\left(\psi_{n},-\frac{1}{2} \nabla^{2} \psi_{n}\right)
$$

becomes negligible as compared to the average potential energy and the mean interaction energy. To show this, we may write the mean single-particle energy (11.48) as

$$
S_{n}=K_{n}+\frac{1}{2} r_{0}^{2}+\frac{1}{2} \nu^{2} z_{0}^{2} .
$$

From the asymptotic expansions (10.65) for $r_{0}$ and $(10.66)$ for $z_{0}$, we have

$$
\frac{1}{2} r_{0}^{2}+\frac{1}{2} \nu^{2} z_{0}^{2} \simeq \frac{3}{4} s^{2 / 5} \quad(s \rightarrow \infty) .
$$

Hence the average kinetic energy

$$
K_{n} \simeq S_{n}-\frac{3}{4} s^{2 / 5} \rightarrow 0 \quad(s \rightarrow \infty)
$$

tends to zero according to the limit (11.50).

If we consider the thermodynamic limit, keeping the frequency $\omega_{r}$ fixed, then

$$
\frac{1}{N}<H>_{N n} \sim N^{2 / 5} \rightarrow \infty \quad(N \rightarrow \infty) .
$$

Then the property $(11.46)$ is not valid for the Hamiltonian $H$, which implies that such a system is thermodynamically unstable. The $N^{7 / 5}$-law of divergence of the average energy (11.54) is the same as that found 372] for the ground-state energy of bosons interacting through Coulomb forces.

Another possibility could be to resort to the thermodynamic limit as defined in Eq. (7.35), when $l_{r} \sim N^{1 / 3}$ and $\omega_{r} \sim N^{-2 / 3}$. Then

$$
\frac{1}{N}<H>_{N n} \sim N^{-2 / 5} \rightarrow 0 \quad(N \rightarrow \infty),
$$

which means that the average energy becomes negligible. 
Finally, we may ask the question, how we should change the frequency $\omega_{r}$ in order to satisfy the condition of thermodynamic stability (11.46) so that the average energy (11.54) would give a finite value,

$$
\frac{1}{N}<H>_{N n} \rightarrow \text { const } \quad(N \rightarrow \infty) ?
$$

The latter is satisfied for $\omega_{r} \sim N^{-1 / 3}$ and, respectively, $l_{r} \sim N^{1 / 6}$. This suggests the definition of the thermodynamic limit as

$$
N \rightarrow \infty, \quad \omega_{r} \rightarrow 0, \quad N \omega_{r}^{3} \rightarrow \text { const } .
$$

It is interesting that the same definition of the thermodynamic limit follows from a quite different condition [31] requiring the finiteness of the Bose-condensation temperature for an ideal gas. 


\section{Chapter 12}

\section{Vortices in Trapped Condensates}

Vortex states in trapped atomic clouds have been considered theoretically by several authors [151, 154, 320, 365, [373]- 375]. Vortex production appears to be a common consequence of mechanically disturbing a condensate. A variety of methods have been suggested by which vortices could be formed. A straightforward way would be by rotating the trap. However, since such a rotation is difficult to realize, other techniques have been proposed: population transfer via a Raman transition into an angular momentum state [162, 376]; creation of circulating states in traps with a multiply connected geometry, such as a toroidal trap or a magnetic trap pinched by a blue-detuned laser [377; stirring the condensate by means of laser beams [378, 379]. The possibility of creating different topological modes, including the vortex ones, by imposing resonance fields has been advanced first in Ref. [139] and studied later in Ref. [380]. Recently, vortices were created in a two-component condensate [9] by combining a microwave field inducing interconversion between the two components at a laser beam rotating with a resonant frequency 142 .

\subsection{Vortex Transition Frequencies}

To transfer a coherent cloud of atoms from the ground state with the energy per particle $E_{000}$ to another coherent state having the energy $E_{n m k}$, one needs to pump into the system the energy per particle

$$
\Omega_{n m k}=E_{n m k}-E_{000}
$$

To estimate the difference (12.1), we may use the optimized approximants of Section 10. These show that for the strong coupling $g \gg 1$ two principally different situations can occur. Since the energy of a coherent state, labelled by the indices $n, m$, and $k$, grows with $g$ as $E_{n m k} \sim g^{2 / 5}$, the difference (12.1) also grows in the same way, $\Omega_{n m k} \sim g^{2 / 5}$, except for the case with the selection rule

$$
(2 \pi)^{3 / 2} \sqrt{2 k+1}(2 n+|m|+1) I_{n m k}=1
$$

when the difference (12.1) diminishes with $g$. The selection rule (12.2) is satisfied for the sole state with $n=0$, $m=1$, and $k=0$, which corresponds to the vortex state with the winding number $m=1$. Vortices with higher winding numbers have essentially higher energies that increase with $g$. The behavior of the vortex energies $\Omega_{n m k}$, where $m \neq 0$, as functions of $\nu g$, is pictured in Fig. 34. As is seen, the energy $\Omega_{010}$ of the basic vortex state with the minimal winding number $m=1$ decreases with $\nu g$ while the energy $\Omega_{020}$ of the vortex state with the winding number $m=2$ first decreases with $\nu g$ and then increases. The qualitatively different behavior of the energy $\Omega_{010}$ of the basic vortex as compared to the energies of other vortex states suggests the following criterion of Energetic Stability: For a given orbital momentum $\hbar|m|$, at large $\nu g$, the creation of $m$ basic vortices is energetically more profitable than the formation of one or several vortices with higher winding numbers giving in total the same orbital momentum. This is in agreement with the thermodynamic stability of vortices studied in Ref. 375. 
To form a vortex in a rotating trap, one has to reach the critical rotation frequency that in dimensionless units reads $\hbar \Omega_{n m k} /\left|L_{z}\right|$, where $L_{z}=\hbar m$ is an eigenvalue of the orbital momentum operator $\hat{L}_{z}=-i \hbar \partial / \partial \varphi$. For the basic vortex with the winding number $m=1$, the critical frequency is

$$
\Omega_{c} \equiv \Omega_{010}=E_{010}-E_{000} .
$$

To consider the dependence of this frequency on the coupling $g$ it is convenient to employ the notation

$$
s \equiv \frac{2 \nu g}{(2 \pi)^{3 / 2}}
$$

and to use the results of Sec. 10. Then, in the weak-coupling limit, we have

$$
\Omega_{c} \simeq 1-\frac{1}{4 \nu^{1 / 2}} s+\frac{3+7 \nu}{64 \nu^{2}} s^{2}-\frac{7+30 \nu+31 \nu^{2}}{512 \nu^{5 / 2}} s^{3}
$$

as $s \rightarrow 0$, and in the strong coupling limit, we get

$$
\Omega_{c} \simeq \frac{3}{2} s^{-2 / 5}-\frac{3}{20}\left(15-2 \nu^{2}\right) s^{-6 / 5}+\frac{3}{20}\left(42-5 \nu^{2}-2 \nu^{4}\right) s^{-2}
$$

as $s \rightarrow \infty$. Invoking the expansion (10.65) for the mean-square radius, according to which $r_{0} \simeq s^{1 / 5}$ as $s \rightarrow \infty$, we may write

$$
\Omega_{c} \simeq \frac{3}{2 r_{0}^{2}} \quad\left(r_{0} \rightarrow \infty\right) .
$$

As a function of $g$, this reduces to

$$
\Omega_{c} \simeq \frac{3\left(2 \pi^{3}\right)^{1 / 5}}{2(\nu g)^{2 / 5}}=\frac{3.424}{(\nu g)^{2 / 5}}
$$

for $\nu g \rightarrow \infty$.

Invoking for the critical rotation frequency the Thomas-Fermi approximation, combined with a hydrodynamic approximation, one finds $320,365,375,381$, the value

$$
\Omega_{c} \approx \frac{5}{2 r_{c}^{2}} \ln \left(0.7 \frac{r_{c}}{\xi_{0}}\right)
$$

where

$$
r_{c}^{2}=2 E_{T F}=\left(\frac{15}{4 \pi} \nu g\right)^{2 / 5}
$$

is the Thomas-Fermi radius and $\xi_{0} \approx 1 / r_{c}$ is the coherence length. From here

$$
\Omega_{c} \approx \frac{0.932}{(\nu g)^{2 / 5}} \ln (0.8 \nu g) .
$$

The expressions (12.7) and (12.10) are close to each other in the region $1 \ll \nu g<10^{3}$. For instance, when $\nu g=100$, their difference is about $10 \%$.

\subsection{Effective Radial Equation}

To analyze the radial cross-section of a vortex, it is convenient to derive an effective radial equation not containing the axial variable $z$. To this end, let us substitute the function

$$
\psi(r, \varphi, z)=\chi(r, z) \frac{e^{i m \varphi}}{\sqrt{2 \pi}},
$$


where $m=0, \pm 1, \pm 2, \ldots$ and $\chi$ is real, into the eigenproblem (9.29) with the Hamiltonian (9.28). Then we have

$$
-\frac{1}{2}\left(\frac{\partial^{2}}{\partial r^{2}}+\frac{1}{r} \frac{\partial}{\partial r}+\frac{\partial^{2}}{\partial z^{2}}\right) \chi+\frac{1}{2}\left(r^{2}+\nu^{2} z^{2}+\frac{m^{2}}{r^{2}}\right) \chi+\frac{g}{2 \pi} \chi^{3}=E \chi .
$$

Let us present $\chi$ as a product

$$
\chi(r, z)=F(r, z) h(z),
$$

in which $F(r, z)$ is a slow function of $z$, such that

$$
\left|\frac{\partial F}{\partial z} h\right| \ll\left|F \frac{d h}{d z}\right|
$$

and where $h$ is normalized according to the condition

$$
\int_{-\infty}^{+\infty} h^{2}(z) d z=1
$$

Define the radial, $E_{r}$, and axial, $E_{z}$, energies by the relation

$$
E \equiv E_{r}+E_{z}, \quad E_{z} \equiv \frac{1}{2} \int_{-\infty}^{+\infty} h(z)\left(-\frac{\partial^{2}}{\partial z^{2}}+\nu^{2} z^{2}\right) h(z) d z .
$$

Then from Eqs. (12.12) and (12.13), taking account of the inequality (12.14), we find

$$
-\frac{1}{2}\left(\frac{\partial^{2}}{\partial r^{2}}+\frac{1}{r} \frac{\partial}{\partial r}\right) F h+\frac{1}{2}\left(r^{2}+\frac{m^{2}}{r^{2}}\right) F h+\frac{g}{2 \pi} F^{3} h^{3}=E_{r} F h .
$$

Introduce the function

$$
f(r) \equiv \int_{-\infty}^{+\infty} F(r, z) h^{2}(z) d z .
$$

Keeping in mind that $F(r, z)$ is a slow function of $z$, we may use the approximation

$$
\int_{-\infty}^{+\infty} F^{3}(r, z) h^{4}(z) d z \cong f^{3}(r) \int_{-\infty}^{+\infty} h^{4}(z) d z .
$$

Multiplying Eq. (12.16) by $h$, integrating over $z$, and defining the radial coupling

$$
\alpha \equiv \frac{g}{2 \pi} \int_{-\infty}^{+\infty} h^{4}(z) d z
$$

and the nonlinear radial Hamiltonian

$$
\hat{H}_{r}(f) \equiv-\frac{1}{2}\left(\frac{d^{2}}{d r^{2}}+\frac{1}{r} \frac{d}{d r}\right)+\frac{1}{2}\left(r^{2}+\frac{m^{2}}{r^{2}}\right)+\alpha f^{2},
$$

we come to the effective radial equation

$$
\hat{H}_{r}(f) f=E_{r} f .
$$

As an example of the function $h$, let us take the harmonic-oscillator wave function

$$
h^{(0)}(z)=\frac{(\nu / \pi)^{1 / 4}}{\sqrt{2^{k} k !}} \exp \left(-\frac{\nu}{2} z^{2}\right) H_{k}(\sqrt{\nu} z) .
$$

Then the axial energy is

$$
E_{z}^{(0)}=\left(k+\frac{1}{2}\right) \nu
$$


and the radial coupling (12.18) becomes

$$
\alpha=\frac{\sqrt{\nu}}{2 \pi} I_{k} g
$$

where

$$
I_{k} \equiv \frac{2}{\pi\left(2^{k} k !\right)^{2}} \int_{0}^{\infty} e^{-2 z^{2}} H_{k}^{4}(z) d z .
$$

The latter integral decreases with $k, e . g$.

$$
I_{0}=\frac{1}{\sqrt{2 \pi}}=0.398942, \quad I_{1}=\frac{3}{4 \sqrt{2 \pi}}=0.299207, \quad I_{2}=\frac{41}{64 \sqrt{2 \pi}}=0.255572 .
$$

Therefore, the radial coupling $\alpha$ diminishes for higher excited states.

The radial equation (12.20) describes the radial profile of a vortex. The angle dependence of the latter, given by Eq. (12.11), defines the circulation velocity

$$
\mathbf{v}=\frac{\hbar m}{m_{0} r} \mathbf{e}_{\varphi},
$$

where $\mathbf{e}_{\varphi}$ is the unit vector corresponding to the polar angle $\varphi$. To be finite, this velocity requires that the winding number be nonzero, $m \neq 0$.

Note that the Thomas-Fermi approximation is not directly applicable for solving Eq. (12.20) in the case of vortex states. This is because the corresponding solution

$$
f_{T F}^{2}(r) \simeq-\frac{m^{2}}{2 \alpha r^{2}} \quad(r \rightarrow 0)
$$

diverges at $r=0$ for $m \neq 0$.

\subsection{Vortex Wave Function}

The structure of the radial Hamiltonian (12.19) shows that there are two qualitatively different regions where either the nonlinear term or the harmonic one is more important as compared to each other. These regions are

$$
\begin{array}{ll}
r \ll \sqrt{\alpha} & \text { (nonlinear) }, \\
r \gg \sqrt{\alpha} & \text { (harmonic). }
\end{array}
$$

When $r \rightarrow \infty$, the harmonic term always prevails. To find an approximate analytic solution to the radial equation (12.20), let us consider two cases, when the coupling is not large and when $\alpha \rightarrow \infty$.

In the first case, when $\alpha$ is not large, say of order one or less, the nonlinear region is small. The radial energy $E_{r}$ can be obtained by the optimized perturbation theory of section 10.1. As the initial approximation, we may take the harmonic Hamiltonian

$$
\hat{H}_{0}=-\frac{1}{2}\left(\frac{d^{2}}{d r^{2}}+\frac{1}{r} \frac{d}{d r}\right)+\frac{1}{2}\left(u^{2} r^{2}+\frac{m^{2}}{r^{2}}\right),
$$

with the eigenvalue

$$
E_{n m}^{(0)}=p u, \quad p \equiv 2 n+|m|+1
$$

and the eigenfunction

$$
f_{n m}^{(0)}(r)=\left[\frac{2 n ! u^{|m|+1}}{(n+|m|) !}\right]^{1 / 2} r^{|m|} \exp \left(-\frac{1}{2} u r^{2}\right) L_{n}^{|m|}\left(u r^{2}\right) .
$$

For the first-order approximation

$$
E_{n m}^{(1)}(\alpha, u)=\left(f_{n m}^{(0)}, \hat{H}_{r} f_{n m}^{(0)}\right)
$$


we find

$$
E_{n m}^{(1)}(\alpha, u)=\frac{p}{2}\left(u+\frac{1}{u}\right)+u I_{n m} \alpha,
$$

where

$$
I_{n m} \equiv \frac{1}{u} \int_{0}^{\infty}\left[f_{n m}^{(0)}(r)\right]^{4} r d r=2\left[\frac{n !}{(n+|m|) !}\right]^{2} \int_{0}^{\infty} x^{2|m|} e^{-2 x}\left[L_{n}^{|m|}(x)\right]^{4} d x .
$$

From the fixed-point condition

$$
\frac{\partial}{\partial u} E_{n m}^{(1)}(\alpha, u)=0
$$

we define the control function

$$
u(s)=\sqrt{\frac{p}{p+s}}, \quad s \equiv 2 I_{n m} \alpha .
$$

For the optimized approximant

$$
E_{r}(s) \equiv E_{n m}^{(1)}(\alpha(s), u(s))
$$

we obtain

$$
E_{r}(s)=\sqrt{p(p+s)} .
$$

In the weak-coupling limit, Eq. 12.30) gives

$$
E_{r}(s) \simeq p+\frac{1}{2} s-\frac{1}{p} s^{2} \quad(s \rightarrow 0)
$$

and in the strong-coupling limit, one has

$$
E_{r}(s) \simeq \sqrt{p}\left(s^{1 / 2}+\frac{p}{2} s^{-1 / 2}-\frac{p^{2}}{8} s^{-3 / 2}\right)
$$

as $s \rightarrow \infty$. It is interesting that if, being based on these asymptotic expansions, we construct the self-similar crossover approximant

$$
E_{1}^{*}(s)=a_{0}(1+A s)^{n_{1}},
$$

as is explained in section 11.1, then

$$
a_{0}=p, \quad A=\frac{1}{p}, \quad n_{1}=\frac{1}{2},
$$

and the crossover formula $(12.33)$ coincides with the energy (12.30).

To find the energy of the basic vortex with the quantum numbers $n=0$ and $m=1$, we note that $I_{00}=1$ and $I_{01}=0.5$. The related control functions, given by Eq. (12.28), are

$$
u_{00}=\frac{1}{\sqrt{1+2 \alpha}}, \quad u_{01}=\sqrt{\frac{2}{2+\alpha}} .
$$

The corresponding radial energies are

$$
E_{00}=\sqrt{1+2 \alpha}, \quad E_{01}=\sqrt{4+2 \alpha} .
$$

Then the vortex energy in the strong-coupling limit is

$$
\Omega_{01} \equiv E_{01}-E_{00} \simeq \frac{3}{2 \sqrt{2}} \alpha^{-1 / 2} \quad(\alpha \rightarrow \infty) .
$$

This is to be compared with the critical rotation frequency

$$
\Omega_{c} \approx \frac{2}{r_{c}^{2}} \ln \left(0.9 \frac{r_{c}}{\xi_{0}}\right)
$$


obtained in the Thomas-Fermi plus hydrodynamic approximations 320] for a two-dimensional vortex.

From the radial equation (12.20), it follows that its solution behaves at small distance as

$$
f(r) \simeq C r^{|m|}\left(1+c_{2} r^{2}+c_{4} r^{4}\right) \quad(r \rightarrow 0)
$$

and at large distance as

$$
f(r) \sim r^{|m|} \exp \left(-\frac{1}{2} r^{2}\right) L_{n}^{|m|}\left(r^{2}\right) \quad(r \rightarrow \infty) .
$$

For the case when $n=0$, we have

$$
f(r) \sim r^{|m|} \exp \left(-\frac{1}{2} r^{2}\right) \quad(r \rightarrow \infty) .
$$

The crossover approximant, sewing the asymptotic expansion (12.34) and (12.36), is

$$
f_{*}(r)=C r^{|m|} \exp \left\{-\frac{1}{2} r^{2}+a r^{2} \exp \left(-b r^{2}\right)\right\},
$$

where $a$ and $b$ are calculated from Eq. (12.20), after the form (12.37) is expanded in powers of $r$ and substituted into this equation. This makes it possible to express the coefficients $a$ and $b$ through $E_{r}$ and $C$. The latter are defined by the equations

$$
E_{r}^{*}=\left(f_{*}, \hat{H}_{r} f_{*}\right), \quad\left(f_{*}, f_{*}\right)=1 .
$$

The accuracy of approximate solutions to Eq. (12.20) can be characterized by the residual

$$
R(r) \equiv\left(\hat{H}_{r}-E_{r}\right) f(r)
$$

and the dispersion

$$
\sigma^{2} \equiv \int_{0}^{\infty}|R(r)|^{2} r d r
$$

Considering the nonrotating case, with $m=0$, we get

$$
a=\frac{1}{2}\left(1+\alpha C^{2}-E_{r}\right), \quad b=\frac{E_{r}^{2}-\alpha^{2} C^{4}-1}{16 a} .
$$

The dispersion (12.40) for the crossover formula 12.37 ) is smaller than that for the variational function 12.24$)$ when $\alpha<70$.

In the case of the basic vortex with the winding number $m=1$, we have

$$
a=1-\frac{E_{r}}{2}, \quad b=\frac{E_{r}^{2}-8 \alpha C^{2}-4}{48 a} .
$$

The crossover function (12.37) is a better approximation than the variational function (12.24) for $\alpha<15$.

Thus, the crossover approximant (12.37) is a reasonable approximation for a vortex wave function if the coupling $\alpha<10$. For large $\alpha \gg 10$, the error of the approximant (12.37), characterized by the residual (12.39) and dispersion (12.40), quickly grows. The reason for this is clear: In constructing the crossover formula (12.37), we have used the information on the behavior of the solution to Eq. (12.20) at small distance, when $r \rightarrow 0$, which is described by the form (12.34), and at large distance, when $r \rightarrow \infty$, where the harmonic term prevails, so that the asymptotic solution is given by Eq. (12.36). At the same time, there is an additional characteristic scale $r \sim \sqrt{\alpha}$ defining the distance at which the dominance of the nonlinear term in the Hamiltonian (12.19) changes to that of the harmonic term. The peculiarity in the behavior of a solution, due to this additional crossover, can be neglected only if $\alpha$ is not large, so that the region $1 \ll r \ll \sqrt{\alpha}$ squeezes to a small interval or practically disappears. The value $\alpha \approx 10$ is exactly that critical value. 
In order to analyze the behavior of the solution to Eq. (12.20) for large coupling $\alpha \gg 10$, let us consider the case, opposite to the previous one, when there exists a wide region $1 \ll r \ll \sqrt{\alpha}$, where the nonlinear term is dominant as compared to the harmonic term. In this region, Eq. (12.20) may be written as

$$
-\frac{1}{2}\left(\frac{d^{2} f}{d r^{2}}+\frac{1}{r} \frac{d f}{d r}\right)+\frac{m^{2}}{2 r^{2}} f+\alpha f^{3}=E_{r} f,
$$

the harmonic term being omitted. To simplify the analysis of Eq. (12.41), we scale it so that it reduces to the equation

$$
\frac{d^{2} f}{d r^{2}}+\frac{1}{r} \frac{d f}{d r}-\frac{m^{2}}{r^{2}} f+f-f^{3}=0 .
$$

The return from Eq. (12.42) back to Eq. (12.41) can be done by the scaling

$$
r \rightarrow \sqrt{2 E_{r}} r, \quad f \rightarrow \sqrt{\frac{\alpha}{E_{r}}} f
$$

To construct a crossover solution in the region $0 \leq r<\sqrt{\alpha}$, when $\alpha \gg 10$, we need an asymptotic expansion for $f(r)$ at $r \rightarrow 0$ and another expansion for $r \gg 1$, but $r<\sqrt{\alpha}$. For example, the ground-state solution, with $m=0$, behaves as

$$
f(r) \simeq C\left(1+c_{2} r^{2}+c_{4} r^{4}\right) \quad(r \rightarrow 0)
$$

at small distance, the coefficients being

$$
c_{2}=\frac{1}{4}\left(C^{2}-1\right), \quad c_{4}=\frac{1}{64}\left(3 C^{4}-4 C^{2}+1\right) .
$$

And for $r \gg 1$, the solution tends to $f(r) \simeq 1$.

Let us concentrate on the vortex solution with $m=1$. Then at small distance, we have

$$
f(r) \simeq C r\left(1+c_{2} r^{2}+c_{4} r^{4}+c_{6} r^{6}\right) \quad(r \rightarrow 0),
$$

where

$$
c_{2}=-\frac{1}{8}, \quad c_{4}=\frac{8 C^{2}+1}{192}, \quad c_{6}=-\frac{80 C^{2}+1}{9216}
$$

At large distance, we find

$$
f(r) \simeq 1-\frac{1}{2} r^{-2}-\frac{9}{8} r^{-4}-\frac{161}{16} r^{-6} \quad(r \gg 1) .
$$

Employing the method of section 11.1, we construct 369 the self-similar crossover approximants

$$
\begin{gathered}
f_{1}^{*}(r)=\frac{1}{2} r\left(1+\frac{1}{4} r^{2}\right)^{-1 / 2}, \\
f_{2}^{*}(r)=\frac{1}{\sqrt{2}} r\left(1+\frac{1}{2} r^{2}+\frac{1}{4} r^{4}\right)^{-1 / 4}, \\
f_{3}^{*}(r)=\frac{1}{4^{1 / 3}} r\left(1+\frac{3}{4} r^{2}+\frac{3}{16} r^{4}+\frac{1}{16} r^{6}\right)^{-1 / 6}, \\
f_{4}^{*}(r)=\frac{1}{136^{1 / 8}} r\left(1+r^{2}+\frac{9}{68} r^{4}+\frac{1}{34} r^{6}+\frac{1}{136} r^{8}\right)^{-1 / 8} .
\end{gathered}
$$

The accuracy of the approximants $(12.45)$ can be checked by comparing them with the exact numerical solution [307, 382 of the vortex equation (12.42). This comparison is presented in Fig. 35, where it is seen that the approximant $f_{4}^{*}(r)$ practically coincides with the numerical solution. 


\section{Chapter 13}

\section{Elementary Collective Excitations}

Following the experimental realization of Bose-Einstein condensate in trapped atomic gases, there has been an intensive study, both experimental and theoretical, of elementary excitations in these systems [30, 31]. For the theoretical description of elementary excitations one usually employs two equivalent schemes. One of them is based on the diagonalization of the Hamiltonian in the Bogolubov approximation [282, 300]. Another approach relies on the linearization of evolution equations. The latter approach can be accomplished in several ways which we illustrate below.

It is worth noting that collective excitations of trapped atoms have many common features with collective excitations in nuclei, that are also finite systems where nucleons are trapped by means of self-consistent potentials [383, 384, and with collective excitations in metallic clusters 385]-387.

\subsection{Linearization of Gross-Pitaevskii Equation}

The Gross-Pitaevskii equation (9.5), when there are no external time-dependent forces, reads

$$
i \hbar \frac{\partial \varphi}{\partial t}=\hat{H}(\varphi) \varphi
$$

with the nonlinear Hamiltonian

$$
\hat{H}(\varphi)=-\frac{\hbar^{2} \nabla^{2}}{2 m_{0}}+U(\mathbf{r})+N A|\varphi|^{2} .
$$

Recall that Eq. (13.1) is an exact equation for the coherent wave function [291. The similar equation (9.10) is an approximate equation for the mean-field order parameter [31.

Collective excitations are described by small oscillations around a stationary solution given by the stationary equation

$$
\hat{H}\left(\varphi_{n}\right) \varphi_{n}(\mathbf{r})=E_{n} \varphi_{n}(\mathbf{r}) .
$$

One usually considers small fluctuations around the ground-state function $\varphi_{0}(\mathbf{r})$, though, in general, one may consider oscillations around a chosen stationary state $\varphi_{n}(\mathbf{r})$.

Let us look for the solution of Eq. (13.1) describing small deviations from a given stationary solution $\varphi_{n}(\mathbf{r})$. To this end, we substitute the function

$$
\varphi(\mathbf{r}, t)=\left[\varphi_{n}(\mathbf{r})+u(\mathbf{r}) e^{-i \omega t}+v^{*}(\mathbf{r}) e^{i \omega t}\right] \exp \left(-\frac{i}{\hbar} E_{n} t\right)
$$

into Eq. (13.1) and linearize the latter with respect to $u(\mathbf{r})$ and $v(\mathbf{r})$. Equating the like terms at the exponentials $\exp (\mp i \omega t)$, we get

$$
\begin{gathered}
{\left[\hat{H}\left(\varphi_{n}\right)-E_{n}+N A\left|\varphi_{n}\right|^{2}-\hbar \omega\right] u+N A \varphi_{n}^{2} v=0,} \\
{\left[\hat{H}\left(\varphi_{n}\right)-E_{n}+N A\left|\varphi_{n}\right|^{2}+\hbar \omega\right] v+N A\left(\varphi_{n}^{*}\right)^{2} u=0 .}
\end{gathered}
$$


This system of coupled equations, sometimes called the Bogolubov-De Gennes equations, defines the eigenvalues $\hbar \omega$ that are the energies of the elementary excitations. For trapped atoms, these equations are usually solved numerically [30, 31].

As an illustration, let us consider the case of a uniform potential $U(\mathbf{r})=U=$ const. For the ground state wave function

$$
\varphi_{0}=\sqrt{n_{0}}, \quad n_{0} \equiv\left|\varphi_{0}(0)\right|^{2},
$$

the stationary equation $(13.3)$ gives the energy

$$
E_{0}=U+\rho_{0} A, \quad \rho_{0} \equiv n_{0} N .
$$

The solutions $u$ and $v$ for the Bogolubov-De Gennes equations (13.5) are plane waves of the form $\exp (i \mathbf{k} \cdot \mathbf{r})$. Then Eq. (13.5) yields

$$
\hbar^{2} \omega^{2}=\frac{\hbar^{2} k^{2}}{2 m_{0}}\left(\frac{\hbar^{2} k^{2}}{2 m_{0}}+2 A \rho_{0}\right) .
$$

This results in the Bogolubov spectrum

$$
\omega_{B}(k)=\sqrt{c^{2} k^{2}+\hbar^{2}\left(\frac{k^{2}}{2 m_{0}}\right)^{2}},
$$

in which

$$
c \equiv \sqrt{\frac{\rho_{0}}{m_{0}} A}
$$

is the sound velocity. In the long-wave limit, the spectrum (13.8) reduces to the acoustic form

$$
\omega_{B}(k) \simeq c k \quad(k \rightarrow 0) .
$$

When the potential $U(\mathbf{r})$ is not a constant, the procedure of calculating the spectrum of elementary excitations is essentially more complicated and is usually done numerically. But by their physical meaning, the corresponding excitations are the analog of phonons.

\subsection{Linearization of Hydrodynamic Equations}

The Gross-Pitaevskii equation (13.1) can be rewritten in the form of hydrodynamic equations. To this purpose, one can present the coherent wave function in terms of a modulus and a phase as

$$
\varphi(\mathbf{r}, t)=\sqrt{n(\mathbf{r}, t)} \exp \{i S(\mathbf{r}, t)\},
$$

where the phase $S$ is real and

$$
n(\mathbf{r}, t)=|\varphi(\mathbf{r}, t)|^{2}
$$

The phase defines the velocity

$$
\mathbf{v}(\mathbf{r}, t) \equiv \frac{\hbar}{m_{0}} \nabla S(\mathbf{r}, t)
$$

so that the density current is

$$
n \mathbf{v}=-\frac{i \hbar}{2 m_{0}}\left(\varphi^{*} \nabla \varphi-\varphi \nabla \varphi^{*}\right)
$$

Substituting the presentation (13.11) into Eq. (13.1) and separating the real and imaginary parts, one obtains the continuity equation

$$
\frac{\partial n}{\partial t}+\nabla \cdot(n \mathbf{v})=0
$$


and the velocity-field equation

$$
m_{0} \frac{\partial \mathbf{v}}{\partial t}+\nabla\left(U+N A n-\frac{\hbar^{2}}{2 m_{0} \sqrt{n}} \nabla^{2} \sqrt{n}+\frac{m \mathbf{v}^{2}}{2}\right)=0 .
$$

Equations (13.15) and (13.16) are completely equivalent to the Gross-Pitaevskii equation (13.1) and are termed the hydrodynamic representation of the latter. If we are interested in the stationary ground-state solutions, then Eqs. (13.15) and (13.16) reduce to

$$
\frac{\partial n_{0}}{\partial t}=0, \quad \mathbf{v}_{0}=0
$$

where

$$
n_{0}=n_{0}(\mathbf{r}) \equiv\left|\varphi_{0}(\mathbf{r})\right|^{2}
$$

and $\varphi_{0}$ satisfies the equation

$$
-\frac{\hbar^{2}}{2 m_{0}} \nabla^{2} \varphi_{0}+\left(U+N A\left|\varphi_{0}\right|^{2}\right) \varphi_{0}=E \varphi_{0} .
$$

To analyze small deviations from the ground-state solutions $n_{0}$ and $\mathbf{v}_{0}$, one writes

$$
n=n_{0}+\delta n, \quad \mathbf{v}=\mathbf{v}_{0}+\delta \mathbf{v} .
$$

Linearizing Eq. (13.15), one gets

$$
\frac{\partial}{\partial t} \delta n+\nabla\left(n_{0} \delta \mathbf{v}\right)=0 .
$$

Linearizing Eq. (13.16), one assumes that $n_{0}$ changes in space much slower than $\delta n$,

$$
\left|\nabla n_{0}\right| \ll|\nabla \delta n| .
$$

Then one finds

$$
m_{0} \frac{\partial}{\partial t} \delta \mathbf{v}+\nabla\left(N A-\frac{\hbar^{2}}{4 m_{0} n_{0}} \nabla^{2}\right) \delta n=0 .
$$

Combining Eqs. (13.19) and (13.21), one comes to the equation

$$
\frac{\partial^{2}}{\partial t^{2}} \delta n=\nabla\left(c^{2} \nabla \delta n\right)-\left(\frac{\hbar}{2 m_{0}} \nabla^{2}\right)^{2} \delta n
$$

in which

$$
c(\mathbf{r}) \equiv \sqrt{\frac{n_{0}(\mathbf{r})}{m_{0}} N A}
$$

is a local sound velocity. For the harmonically oscillating $\delta n$, say, as $\cos \omega t$, one has

$$
\omega^{2} \delta n+\nabla\left(c^{2} \nabla \delta n\right)=\left(\frac{\hbar}{2 m_{0}} \nabla^{2}\right)^{2} \delta n .
$$

Note that for the uniform case, when $c=$ const and $\delta n \sim \cos \mathbf{k} \cdot \mathbf{r}$, we return back to the Bogolubov spectrum (13.8). However, for the nonuniform case, the local sound velocity $(13.23)$ depends on the space variable $\mathbf{r}$. For low-lying excitations, one may neglect the right-hand side of Eq. (13.24), which gives

$$
\omega^{2} \delta n+\nabla\left(c^{2} \nabla \delta n\right)=0 .
$$

For a spherical trap, and using the Thomas-Fermi approximation for $n_{0}(\mathbf{r})$, the solutions to this equation can be presented as

$$
\delta n(\mathbf{r})=P_{2 n}^{l}(r) r^{l} Y_{l m}(\vartheta, \varphi),
$$


where $P_{2 n}^{l}$ are even polynomials of degree $2 n ; Y_{l m}$ are spherical functions; and $n, l, m$ are quantum numbers. The dispersion law is given by the Stringari spectrum [133]

$$
\omega_{n l}=\omega_{0}\left(2 n^{2}+2 n l+3 n+l\right)^{1 / 2} .
$$

For cylindrically symmetric traps, analytical solutions for the spectrum of elementary excitations are available only for some particular modes [31]. For instance, the scissors mode, generated by a sudden rotation of the confining trap 146, has the frequency

$$
\omega=\sqrt{2} \omega_{0},
$$

with the Thomas-Fermi approximation being again involved.

\subsection{Lagrangian Variational Technique}

For solving complicated nonlinear differential equations in partial derivatives, a variational technique has been widely used [388], which provides approximate solutions to such equations. The basic idea of this variational method is to take a trial function with a fixed shape but some free parameters in order to reduce the infinitedimensional dynamical system of partial differential equations to a set of ordinary differential equations for the variational parameters that characterize the solution. This technique has also been applied [389] to solve the time-dependent Gross-Pitaevskii equation and to calculate collective-excitation frequencies.

The first step of the method is to formulate a variational problem that yields the considered differential equation. This can be formulated as the problem of extremizing an action

$$
\delta \int L(t) d t=0
$$

in which the Lagrangian

$$
L(t)=\int \mathcal{L}(\mathbf{r}, t) d \mathbf{r}
$$

is expressed through the Lagrangian density. In our case, the latter is

$$
\mathcal{L}(\mathbf{r}, t)=-\frac{i}{2} \hbar\left(\varphi^{*} \frac{\partial \varphi}{\partial t}-\frac{\partial \varphi^{*}}{\partial t} \varphi\right)+\frac{\hbar^{2}}{2 m_{0}}|\nabla \varphi|^{2}+U|\varphi|^{2}+\frac{1}{2} N A|\varphi|^{4}
$$

As is easy to check, the extremum condition (13.26), resulting in the Lagrangian equation

$$
\frac{d}{d t} \frac{\delta L}{\delta \dot{\varphi}}-\frac{\delta L}{\delta \varphi}=0
$$

where $\dot{\varphi} \equiv d \varphi / d t$, for the Lagrangian density (13.28), yields the Gross-Pitaevskii equation (13.1).

For the general anisotropic confining potential, it is convenient to pass to dimensionless quantities as defined in section 9.3 and also to measure time in units of $\omega_{0}^{-1}$. The return to the dimensional notation is done by the substitution

$$
\mathcal{L}(\mathbf{x}, t) \rightarrow \frac{\mathcal{L}(\mathbf{r}, t)}{\hbar \omega_{0}}, \quad t \rightarrow \omega_{0} t .
$$

The dimensionless Lagrangian density is

$$
\mathcal{L}(\mathbf{x}, t)=-\frac{i}{2}\left(\psi^{*} \frac{\partial \psi}{\partial t}-\frac{\partial \psi^{*}}{\partial t} \psi\right)+\frac{1}{2} \sum_{i=1}^{3}\left(\left|\frac{\partial \psi}{\partial x_{i}}\right|^{2}+\omega_{i}^{2} x_{i}^{2}\right)+\frac{1}{2} g|\psi|^{4} .
$$

For a while, there were no approximations, so that all transformations are exact.

Now, instead of varying the action with respect to $\psi$ and $\psi^{*}$, let us present the sought solution in the Gaussian form

$$
\psi(\mathbf{x}, t)=\prod_{i=1}^{3} \psi_{i}\left(x_{i}, t\right),
$$




$$
\psi_{i}\left(x_{i}, t\right)=C_{i}(t) \exp \left\{-\frac{u_{i}(t)}{2}\left[x_{i}-a_{i}(t)\right]^{2}+i \alpha_{i}(t) x_{i}+i \beta_{i}(t) x_{i}^{2}\right\} .
$$

From the normalization $\left(\psi_{i}, \psi_{i}\right)=1$, we have

$$
\left|C_{i}(t)\right|=\left[\frac{u_{i}(t)}{\pi}\right]^{1 / 4}
$$

Then, with the ansatz (13.31), we calculate the Lagrangian (13.27), which can be done explicitly because of the Gaussian dependence of the trial function (13.31) on the space variable $x_{i}$. After this, we assume that the set of yet unknown trial functions $u_{i}(t), a_{i}(t), \alpha_{i}(t)$, and $\beta_{i}(t)$, where $i=1,2,3$, satisfies the Lagrange equation

$$
\frac{d}{d t} \frac{\partial L}{\partial \dot{q}}-\frac{\partial L}{\partial q}=0
$$

in which $q(t)$ is any function from the given trial set. This assumption reduces the infinite-dimensional problem of solving Eq. (13.1) to a finite-dimensional problem of ordinary differential equations. As is clear, the ansatz (13.31), together with the assumption (13.32), defines approximate solutions to Eq. (13.1), whose accuracy cannot be controlled.

Note that the described reduction of the partial differential equations could be done as well for a timedependent trapping potential $U(\mathbf{r}, t)$ in the Hamiltonian (13.2). Since, till now, we have nowhere used any linearization procedure, the reduced set of equations can, in general, describe nonlinear motion.

From the set of equations (13.32), one can derive the equations

$$
\ddot{a}_{i}+\omega_{i}^{2} a_{i}=0
$$

for the center-of-condensate variables that harmonically oscillate with the bare frequencies $\omega_{i}$. The oscillations of the atomic-cloud shape are characterized by the frequencies $u_{i}$, for which we get the equations

$$
\ddot{u}_{i}-\frac{3 \dot{u}_{i}^{2}}{2 u_{i}}+2 u_{i}\left(u_{i}^{2}-\omega_{i}^{2}\right)+s u_{i}^{2} \sqrt{u_{1} u_{2} u_{3}}=0,
$$

where the standard notation

$$
s \equiv \frac{2 g}{(2 \pi)^{3 / 2}}
$$

is employed. Introducing the effective cloud widths

$$
b_{i}(t) \equiv \frac{1}{\sqrt{u_{i}(t)}},
$$

one may transform Eq. (13.34) to the form

$$
\ddot{b}_{i}+\omega_{i}^{2} b_{i}=\frac{s}{2 b_{i} b_{1} b_{2} b_{3}}+\frac{1}{b_{i}^{3}} .
$$

The stationary solutions to Eq. (13.36) are given by the equation

$$
\omega_{i}^{4}\left(b_{i}^{*}\right)^{4}=\frac{s\left(b_{i}^{*}\right)^{2}}{2 b_{1}^{*} b_{2}^{*} b_{3}^{*}}+1 .
$$

In order to find the frequencies of collective excitations, one has to consider small deviations of the variables $b_{i}$ near their stationary points $b_{i}^{*}$. To this end, one substitutes

$$
b_{i}=b_{i}^{*}+\delta b_{i}
$$

into Eq. (13.36) and linearizes the latter with respect to $\delta b_{i}$. This results in a system of three differential linear equations whose harmonic solutions, say of the form $\cos \omega t$, give an algebraic system of equations. Equating the 
determinant of the latter system to zero, one comes to an equation for the spectrum of collective excitations. For example, following this procedure in the case of an isotropic trapping potential, when $\omega_{i}=1, b_{i}^{*}=b^{*}$, and neglecting the term 1 in the right-hand side of Eq. (13.37), which assumes that $s \gg 1$, so that

$$
b^{*}=\left(\frac{s}{2}\right)^{1 / 5},
$$

we obtain the system

$$
\begin{aligned}
& \left(\omega^{2}-3\right) \delta b_{1}-\delta b_{2}-\delta b_{3}=0, \\
& \delta b_{1}-\left(\omega^{2}-3\right) \delta b_{2}+\delta b_{3}=0, \\
& \delta b_{1}+\delta b_{2}-\left(\omega^{2}-3\right) \delta b_{3}=0 .
\end{aligned}
$$

Equating the determinant to zero yields

$$
\left(\omega^{2}-3\right)^{2}-3\left(\omega^{2}-3\right)-2=0 .
$$

This leads to the spectrum

$$
\varepsilon_{1,2}=\sqrt{2}, \quad \varepsilon_{3}=\sqrt{5} .
$$

The equations for the effective cloud widths $b_{i}$, similar to Eq. (13.36), can also be derived [31] from the hydrodynamic equations (13.15) and (13.16), by assuming the harmonic dependence of the density $n(\mathbf{r}, t)$ on the space coordinates and a special form of the velocity $\mathbf{v}(\mathbf{r}, t)$. 


\section{Chapter 14}

\section{Multicomponent Bose Mixtures}

Multicomponent systems of trapped Bose-Einstein condensates have been realized for rubidium in a magnetic trap [7, 123] and for sodium in an optical trap [78, 125, 126]. There exists a number of works with theoretical treatment of such systems (see [7], 390]-392] and references therein).

\subsection{Coherent States of Mixtures}

The Hamiltonian of a multicomponent Bose mixture has the form

$$
\begin{gathered}
H=\sum_{i} \int \psi_{i}^{\dagger}(\mathbf{r}, t)\left[-\frac{\hbar^{2} \nabla^{2}}{2 m_{i}}+U_{i}(\mathbf{r}, t)\right] \psi_{i}(\mathbf{r}, t) d \mathbf{r}+ \\
+\frac{1}{2} \sum_{i j} \int \psi_{i}^{\dagger}(\mathbf{r}, t) \psi_{j}^{\dagger}\left(\mathbf{r}^{\prime}, t\right) \Phi_{i j}\left(\mathbf{r}-\mathbf{r}^{\prime}\right) \psi_{j}\left(\mathbf{r}^{\prime}, t\right) \psi_{i}(\mathbf{r}, t) d \mathbf{r} d \mathbf{r}^{\prime},
\end{gathered}
$$

in which the index $i=1,2 \ldots$ enumerate the components; $m_{i}$ is a mass; $U_{i}(\mathbf{r}, t)$ is an external field including the trapping potential; the interaction potential $\Phi_{i j}$ has the symmetry properties

$$
\Phi_{i j}(\mathbf{r})=\Phi_{i j}(-\mathbf{r})=\Phi_{j i}(\mathbf{r}) ;
$$

and $\psi_{i}(\mathbf{r}, t)$ are field operators satisfying the Bose commutation relations,

$$
\left[\psi_{i}(\mathbf{r}, t), \psi_{j}^{\dagger}\left(\mathbf{r}^{\prime}, t\right)\right]=\delta_{i j} \delta\left(\mathbf{r}-\mathbf{r}^{\prime}\right) .
$$

The evolution equations for the field operators are given by the Heisenberg equations that can be written in one of two equivalent forms: in the commutator form

$$
i \hbar \frac{\partial}{\partial t} \psi_{j}(\mathbf{r}, t)=\left[\psi_{j}(\mathbf{r}, t), H\right]
$$

or in the variational representation

$$
i \hbar \frac{\partial}{\partial t} \psi_{j}(\mathbf{r}, t)=\frac{\delta H}{\delta \psi_{j}^{\dagger}(\mathbf{r}, t)} .
$$

Any of these representations yield the same equation

$$
i \hbar \frac{\partial}{\partial t} \psi_{j}(\mathbf{r}, t)=H_{j}(\psi) \psi_{j}(\mathbf{r}, t),
$$

in which

$$
H_{j}(\psi)=-\frac{\hbar^{2} \nabla^{2}}{2 m_{j}}+U_{j}(\mathbf{r}, t)+\sum_{i} \int \Phi_{i j}\left(\mathbf{r}-\mathbf{r}^{\prime}\right) \psi_{i}^{\dagger}\left(\mathbf{r}^{\prime}, t\right) \psi_{i}\left(\mathbf{r}^{\prime}, t\right) d \mathbf{r}^{\prime}
$$


Coherent states can be defined as is described in Section 8, with a straightforward generalization for a mixture. The coherent state $h_{i}$ for the $i$-component is an eigenvalue of the destruction operator $\psi_{i}$, so that

$$
\psi_{i}(\mathbf{r}, t) h_{i}=\eta_{i}(\mathbf{r}, t) h_{i} .
$$

The coherent state for a multicomponent system is given by the tensor product

$$
h=\bigotimes_{i} h_{i} .
$$

The action of an operator $\psi_{i}$ on the state $h$ is defined as

$$
\psi_{i}(\mathbf{r}, t) \bigotimes_{j} \hat{1}_{j} h=\eta_{i}(\mathbf{r}, t) h .
$$

Multiplying Eq. (14.2) from the left by $h^{+}$and from the right by $h$, we obtain the evolution equation

$$
i \hbar \frac{\partial}{\partial t} \eta_{j}(\mathbf{r}, t)=H_{j}(\eta) \eta_{j}(\mathbf{r}, t)
$$

for the coherent field $\eta_{j}(\mathbf{r}, t)$, with the effective nonlinear Hamiltonian

$$
H_{j}(\eta)=-\frac{\hbar^{2} \nabla^{2}}{2 m_{j}}+U_{j}(\mathbf{r}, t)+\sum_{i} \int \Phi_{i j}\left(\mathbf{r}-\mathbf{r}^{\prime}\right)\left|\eta_{i}\left(\mathbf{r}^{\prime}, t\right)\right|^{2} d \mathbf{r}^{\prime} .
$$

By means of the notation

$$
\eta_{i}(\mathbf{r}, t)=\sqrt{N_{i}} \varphi_{i}(\mathbf{r}, t)
$$

we may introduce the coherent field $\varphi_{i}$ normalized to unity, $\left(\varphi_{i}, \varphi_{i}\right)=1$, so that $N_{i}$ plays the role of the number of particles in the $i$-component. Then, for the normalized coherent field, the evolution equation is

$$
i \hbar \frac{\partial}{\partial t} \varphi_{j}(\mathbf{r}, t)=\hat{H}_{j}(\varphi) \varphi_{j}(\mathbf{r}, t)
$$

with the nonlinear Hamiltonian

$$
\hat{H}_{j}(\varphi)=-\frac{\hbar^{2} \nabla^{2}}{2 m_{j}}+U_{j}(\mathbf{r}, t)+\sum_{i} N_{i} \int \Phi_{i j}\left(\mathbf{r}-\mathbf{r}^{\prime}\right)\left|\varphi_{i}\left(\mathbf{r}^{\prime}, t\right)\right|^{2} d \mathbf{r}^{\prime} .
$$

In the case of a dilute system of atoms, whose scattering lengths $a_{i j}$ satisfy the inequality

$$
\frac{\left|a_{i j}\right|}{a} \ll 1
$$

in which $a$ is the mean interatomic distance, one may model the interaction potential by the Fermi pseudopotential

$$
\Phi_{i j}(\mathbf{r})=A_{i j} \delta(\mathbf{r}),
$$

with the interaction amplitude

$$
A_{i j}=2 \pi \hbar^{2} \frac{a_{i j}}{m_{i j}}, \quad m_{i j} \equiv \frac{m_{i} m_{j}}{m_{i}+m_{j}} .
$$

By assumption, $a_{i j}=a_{j i}$, hence $A_{i j}=A_{j i}$. Then the nonlinear Hamiltonian (14.9) becomes

$$
\hat{H}_{j}(\varphi)=-\frac{\hbar^{2} \nabla^{2}}{2 m_{j}}+U_{j}(\mathbf{r}, t)+\sum_{i} N_{i} A_{i j}\left|\varphi_{i}\right|^{2}
$$

The stationary solutions of Eq. (14.8), when the external potential $U_{j}(\mathbf{r})$ does not depend on time, can be presented as

$$
\varphi_{j}(\mathbf{r}, t)=\varphi_{j}(\mathbf{r}) \exp \left(-\frac{i}{\hbar} E_{j} t\right),
$$

with the coherent modes $\varphi_{j}(\mathbf{r})$ defined by the eigenproblem

$$
\hat{H}_{j}(\varphi) \varphi_{j}(\mathbf{r})=E_{j} \varphi_{j}(\mathbf{r}) .
$$

Note that, in the same way as for single-component systems, the evolution equation (14.8) is an exact equation for the coherent field $\varphi_{j}(\mathbf{r}, t)$. So is the eigenproblem (14.15) for the coherent mode $\varphi_{j}(\mathbf{r})$ in the stationary case. 


\subsection{Branching of Excitation Spectrum}

The spectrum of collective excitations for a multicomponent Bose mixture can be defined by means of the same methods as for a one-component system, as is described in Chapter 13. For instance, one may linearize the nonlinear equation (14.8) after substituting there

$$
\varphi_{j}(\mathbf{r}, t)=\left[\varphi_{j}(\mathbf{r})+u_{j}(\mathbf{r}) e^{-i \omega t}+v_{j}^{*}(\mathbf{r}) e^{i \omega t}\right] \exp \left(-\frac{i}{\hbar} E_{j} t\right) .
$$

The linearization with respect to the functions $u_{j}$ and $v_{j}$ yields the system of equations

$$
\begin{aligned}
& \left(\hbar \omega-\hat{H}_{j}(\varphi)+E_{j}\right) u_{j}-\sum_{i} N_{i} A_{i j}\left(\varphi_{i}^{*} u_{i}+\varphi_{i} v_{i}\right) \varphi_{j}=0, \\
& \left(\hbar \omega+\hat{H}_{j}(\varphi)-E_{j}\right) v_{j}+\sum_{i} N_{i} A_{i j}\left(\varphi_{i}^{*} u_{i}+\varphi_{i} v_{i}\right) \varphi_{j}^{*}=0,
\end{aligned}
$$

where $\hat{H}_{j}(\varphi)$ is defined on stationary solutions $\varphi_{i}(\mathbf{r})$.

For a nonuniform system, Eqs. (14.17) are to be solved numerically. In order to demonstrate the main specific features distinguishing the case of a mixture from that of a single-component system, let us consider the uniform case, when the external potential $U_{j}=$ const. This case can be treated as a uniform approximation for a large trap. In this approximation, the ground-state stationary solutions of Eq. (14.15) can be written as

$$
\varphi_{j}(\mathbf{r})=\sqrt{n_{j}}, \quad n_{j} \equiv\left|\varphi_{j}(0)\right|^{2}
$$

and the corresponding energies as

$$
E_{j}=U_{j}+\sum_{i} n_{i} N_{i} A_{i j}
$$

Then $u_{j}$ and $v_{j}$ are presented by plane waves of the form $e^{i \mathbf{k} \cdot \mathbf{r}}$. Therefore, we have

$$
\left(\hat{H}_{j}(\varphi)-E_{j}\right) u_{j}=K_{j} u_{j}, \quad K_{j} \equiv \frac{\hbar^{2} k^{2}}{2 m_{j}}
$$

and a similar relation for $v_{j}$. Using this, we may present Eqs. (14.17) in the form

$$
\begin{aligned}
& \left(\hbar \omega-K_{j}\right) u_{j}-\sum_{i} \sqrt{n_{i} n_{j}} N_{i} A_{i j}\left(u_{i}+v_{i}\right)=0 \\
& \left(\hbar \omega+K_{j}\right) v_{j}+\sum_{i} \sqrt{n_{i} n_{j}} N_{i} A_{i j}\left(u_{i}+v_{i}\right)=0 .
\end{aligned}
$$

To simplify the consideration, let us analyze the case of a two-component mixture. Then Eqs. (14.20) form a system of four linear algebraic equations. The condition of having nontrivial solutions is that the determinant be zero. This condition can be presented as

$$
|\hat{D}-\hbar \omega \hat{1}|=0
$$

where $\hat{D}$ is the dynamical matrix, whose elements are

$$
\begin{array}{cl}
D_{11}=K_{1}+n_{1} N_{1} A_{11}, & D_{12}=\sqrt{n_{1} n_{2}} N_{2} A_{12}, \quad D_{13}=n_{1} N_{1} A_{11}, \quad D_{14}=D_{12}, \\
D_{21}=\sqrt{n_{1} n_{2}} N_{1} A_{12}, & D_{22}=K_{2}+n_{2} N_{2} A_{22}, \quad D_{23}=D_{21}, \quad D_{24}=n_{2} N_{2} A_{22}, \\
D_{31}=-D_{13}, \quad & D_{32}=-D_{12}, \quad D_{33}=-D_{11}, \quad D_{34}=-D_{12}, \\
D_{41}=-D_{21}, \quad & D_{42}=-D_{24}, \quad D_{43}=-D_{21}, \quad D_{44}=-D_{22} .
\end{array}
$$


To write down Eq. (14.21) explicitly, it is convenient to introduce the following notation:

$$
\omega_{i}^{2}(k) \equiv c_{i}^{2} k^{2}+\hbar^{2}\left(\frac{k^{2}}{2 m_{i}}\right)^{2},
$$

which would define a single-component spectrum with the sound velocity

$$
c_{i} \equiv \sqrt{\frac{\rho_{i}}{m_{i}} A_{i i}}, \quad \rho_{i} \equiv n_{i} N_{i} ;
$$

and let us also denote

$$
\omega_{12}^{2}(k) \equiv c_{12}^{2} k^{2}
$$

with

$$
c_{12}^{2} \equiv \sqrt{\frac{\rho_{1} \rho_{2}}{m_{1} m_{2}}} A_{12} .
$$

Then from Eq. (14.21), we find

$$
\left(\omega^{2}-\omega_{1}^{2}\right)\left(\omega^{2}-\omega_{2}^{2}\right)=\omega_{12}^{4} .
$$

The latter equation yields for the spectrum

$$
\omega_{ \pm}^{2}(k)=\frac{1}{2}\left[\omega_{1}^{2}+\omega_{2}^{2} \pm \sqrt{\left(\omega_{1}^{2}-\omega_{2}^{2}\right)^{2}+4 \omega_{12}^{4}}\right] .
$$

This means that, instead of one branch (13.8) for the spectrum of collective excitations of a single-component system, we now have a two-branch spectrum, given by Eq. (14.27) for a two-component mixture. In general, for an $n$-component mixture, we should have $n$ branches of the excitation spectrum.

In the long-wave limit, Eq. (14.27) gives two acoustic branches

$$
\omega_{ \pm}(k) \simeq c_{ \pm} k \quad(k \rightarrow 0)
$$

with the corresponding sound velocities $c_{ \pm}$defined by the expression

$$
c_{ \pm}^{2} \equiv \frac{1}{2}\left[c_{1}^{2}+c_{2}^{2} \pm \sqrt{\left(c_{1}^{2}-c_{2}^{2}\right)^{2}+4 c_{12}^{4}}\right] .
$$

In the short-wave limit, one has two single-particle branches

$$
\omega_{ \pm}(k) \simeq \frac{k^{2}}{2 m_{j}} \quad(k \rightarrow \infty) .
$$

The two branches of the collective spectrum of a two-component mixture can be interpreted in the following way. One branch, $\omega_{+}(k)$, describes the oscillation of the total density of the mixture, when both components move together. And another branch, $\omega_{-}(k)$, characterizes the relative fluctuations of the components with respect to each other. It is worth noting that neither of the sound velocities $c_{ \pm}$coincides with the hydrodynamic sound velocity $c$ defined by the derivative $c^{2} \equiv \partial P / \partial \rho_{m}$, in which $P$ is pressure and $\rho_{m}$ is mass density.

\subsection{Dynamic and Thermodynamic Stability}

The mixture of trapped atoms is not always stable and it may stratify into subsystems of pure one-component phases [7], 390] -[392] in the same way as it happens for uniform Bose mixtures [393]- 395]. The criteria of stability can be separated into dynamic and thermodynamic ones.

The system is dynamically stable if its spectrum of collective excitations is positive everywhere, except for a countable number of points where it is zero. For a uniform two-component Bose mixture, the condition of dynamic stability is

$$
\omega_{ \pm}(k)>0
$$


With the spectrum (14.27), this gives

$$
\omega_{1}^{2}(k) \omega_{2}^{2}(k)>\omega_{12}^{4}(k) .
$$

Since $\omega_{-}(k) \leq \omega_{+}(k)$, we actually need to analyze stability only for the branch $\omega_{-}(k)$. This branch describes relative oscillations of components with respect to each other. When $\omega_{-}(k)$ becomes negative, the mixture is unstable with respect to the stratification of the components.

In the long-wave limit, $k \rightarrow 0$, the inequality (14.32) reduces to

$$
c_{1}^{2} c_{2}^{2}>c_{12}^{4} .
$$

Using Eqs. (14.23) and (14.25), we have

$$
A_{11} A_{22}>A_{12}^{2} \text {. }
$$

Taking account of the relation (14.12), we find the condition of dynamic stability for the scattering lengths,

$$
a_{11} a_{22}>\frac{\left(m_{1}+m_{2}\right)^{2}}{4 m_{1} m_{2}} a_{12}^{2} .
$$

The equality

$$
\frac{a_{11} a_{22}}{a_{12}^{2}}=\frac{\left(m_{1}+m_{2}\right)^{2}}{4 m_{1} m_{2}}
$$

defines the stratification boundary. It is interesting that the condition of dynamic stability is the same for uniform as well as for trapped mixtures [391, 392].

The system is thermodynamically stable if its free energy is minimal. At zero temperature, free energy coincides with internal energy. Here, we consider the case when the whole system is in a coherent state, so that we need to compare the average energies of the given coherent state for the mixture and for the stratified system. The Hamiltonian of the latter writes

$$
\begin{gathered}
H_{s t r}=\sum_{i} H_{i} \quad V=\sum_{i} V_{i}, \\
H_{i}=\int_{V_{i}} \psi_{i}^{\dagger}(\mathbf{r}, t)\left[-\frac{\hbar^{2} \nabla^{2}}{2 m_{i}}+U_{i}(\mathbf{r}, t)\right] \psi_{i}(\mathbf{r}, t) d \mathbf{r}+ \\
+\frac{1}{2} \int_{V_{i}} \psi_{i}^{\dagger}(\mathbf{r}, t) \psi_{i}^{\dagger}\left(\mathbf{r}^{\prime}, t\right) \Phi_{i i}\left(\mathbf{r}-\mathbf{r}^{\prime}\right) \psi_{i}\left(\mathbf{r}^{\prime}, t\right) \psi_{i}(\mathbf{r}, t) d \mathbf{r} d \mathbf{r}^{\prime} .
\end{gathered}
$$

This describes independent pure components separated in their own regions of space.

For simplicity, we again employ the uniform approximation when the coherent field for each component can be written as

$$
\varphi_{i}(\mathbf{r})=\left\{\begin{array}{lc}
V^{-1 / 2} & (\text { mixed }) \\
V_{i}^{-1 / 2} & (\text { stratified })
\end{array}\right.
$$

according to the case of a mixed or stratified system, respectively. For a two-component system, one has

$$
N=N_{1}+N_{2}, \quad V=V_{1}+V_{2} .
$$

We assume that all particles $N_{1}$ and $N_{2}$ are in their coherent states characterized by the ground-state coherent fields (14.38). Then the quantum coherent average of the Hamiltonian (14.1) gives the energy of the mixture

$$
<H>_{N}=N_{1} U_{1}+N_{2} U_{2}+\frac{N_{1}^{2} A_{11}+N_{2}^{2} A_{22}+2 N_{1} N_{2} A_{12}}{2\left(V_{1}+V_{2}\right)} .
$$

And the quantum coherent average of the Hamiltonian (14.37) yields the energy

$$
<H_{s t r}>_{N}=N_{1} U_{1}+N_{2} U_{2}+\frac{N_{1}^{2} A_{11}}{2 V_{1}}+\frac{N_{2}^{2} A_{22}}{2 V_{2}}
$$


of the stratified system.

The condition of thermodynamic stability is

$$
\left(<H>_{N}-<H_{s t r}>_{N}\right)<0 .
$$

From here, we find

$$
N_{1}^{2} V_{2}^{2} A_{11}+N_{2}^{2} V_{1}^{2} A_{22}-2 N_{1} N_{2} V_{1} V_{2} A_{12}>0 .
$$

In an important case, when $A_{11}$ and $A_{22}$ are positive, condition (14.43) reduces to

$$
\left(N_{1} V_{2} \sqrt{A_{11}}-N_{2} V_{1} \sqrt{A_{22}}\right)^{2}+2 N_{1} N_{2} V_{1} V_{2}\left(\sqrt{A_{11} A_{22}}-A_{12}\right)>0
$$

To be stable in general, any system has to be both dynamically as well as thermodynamically stable, so that both conditions (14.34) and (14.43) be valid. Let us analyze the relation between these conditions for different particular cases:

(i) $A_{11}, A_{22}, A_{12}>0$. The condition of dynamic stability (14.34) is stronger than that of thermodynamic stability (14.43) in the sense that from the former the latter follows. Dynamic stability is sufficient for thermodynamic stability, although not necessary. A system can be thermodynamically stable, but not dynamically stable. Conversely, thermodynamic instability yields dynamic instability.

(ii) $A_{11}, A_{22}>0, A_{12}<0$. The mixture is always thermodynamically stable, but not necessarily dynamically stable.

(iii) $A_{11}$ and $A_{22}$ are of different signs, while $A_{12}$ is of arbitrary sign. The system is never dynamically stable, although it can be thermodynamically stable.

(iv) $A_{11}, A_{22}<0, A_{12}>0$. The system is never thermodynamically stable, but can be dynamically stable.

(v) $A_{11}, A_{22}, A_{12}<0$. Then inequality (14.43) can be transformed into

$$
\left(N_{1} V_{2} \sqrt{\left|A_{11}\right|}-N_{2} V_{1} \sqrt{\left|A_{22}\right|}\right)^{2}+2 N_{1} N_{2} V_{1} V_{2}\left(\sqrt{\left|A_{11} A_{22}\right|}-\left|A_{12}\right|\right)<0 .
$$

The latter inequality cannot be compatible with condition (14.34), so that dynamic stability leads to thermodynamic instability and thermodynamic stability provokes dynamic instability.

Summarizing this analysis, we conclude that the two-component Bose mixture is both dynamically and thermodynamically stable provided that

$$
A_{11}>0, \quad A_{22}>0,
$$

and condition (14.34) is valid.

Here, we have analyzed the conditions of stability for a coherent mixture. The same conditions can be obtained for a liquid mixture with broken gauge symmetry, when only a fraction of atoms are in the Bose condensate [393, 394], and also for a normal mixture of Bose liquids without broken gauge symmetry [395].

The first experiments [7, 123] involving multiple-species condensates were performed with ${ }^{87} \mathrm{Rb}$ atoms evaporatively cooled in the $\mid F=2, m_{F}=2>$ and $\mid 1,-1>$ spin states. The scattering lengths, known at the $1 \%$ level, are in the proportion $a_{11}: a_{12}: a_{22}=1.03: 1: 0.97$, with the average of three being $55 \AA$ [122. For equal masses $m_{1}=m_{2}$, the stability condition (14.35) reads $a_{11} a_{22}>a_{12}^{2}$. In the case of ${ }^{87} \mathrm{Rb}$, one has $a_{11} a_{22} / a_{12}^{2}=0.9991<1$. Hence, these two condensates cannot compose a uniform mixture. The Bose condensates of sodium [78, 125, 126] in two different internal states $\mid F=1, m_{F}=0>$ and $\mid 1,1>$ have the scattering lengths $a_{11}=a_{12}=2.75 \times 10^{-9} \mathrm{~cm}$ and $a_{22}=2.65 \times 10^{-9} \mathrm{~cm}$. From here, one has $a_{11} a_{22} / a_{12}^{2}=0.964<1$, thence these condensates cannot be mixed. 


\subsection{Stratification of Moving Components}

It is possible to experimentally create a binary mixture of trapped Bose-Einstein condensates with a relative motion of components 123. The presence of such a motion should impose additional constraints on the stability of a mixture.

Consider a coherent mixture of components, each of which moves with a constant linear velocity $\mathbf{V}_{j}$. For the sake of simplicity, let us again invoke the uniform approximation, when the ground-state coherent field of an immovable component is $\sqrt{n_{j}}$. Then for a moving component, because of the Galilean transformation, one has

$$
\varphi_{j}(\mathbf{r})=\sqrt{n_{j}} e^{i \mathbf{k}_{j} \cdot \mathbf{r}}, \quad \mathbf{k}_{j} \equiv \frac{m_{j}}{\hbar} \mathbf{V}_{j}
$$

The eigenvalue of Eq. (14.15) becomes

$$
E_{j}=U_{j}+\sum_{i} \rho_{i} A_{i j}+\frac{1}{2} m_{j} \mathbf{V}_{j}^{2}
$$

where $\rho_{i} \equiv n_{i} N_{i}$. As compared to the ground-state energy (14.19), we have here an additional term corresponding to the kinetic energy of motion. Similarly, for the coherent averages of the Hamiltonians (14.1) and (14.37), we would obtain the expressions (14.40) and (14.41) with additive terms $\frac{1}{2} \sum_{j} m_{j} V_{j}^{2}$. These terms cancel each other when analyzing the condition (14.42). Hence, thermodynamic stability is not affected by such a motion of components.

To check dynamic stability, we need to find the spectrum of collective excitations given by the frequencies $\omega$ satisfying Eqs. (14.17). The solutions $u_{j}$ and $v_{j}$ to the latter equations, in the case of the coherent fields (14.46), depend on the space variable as

$$
u_{j} \sim e^{i\left(\mathbf{k}+\mathbf{k}_{j}\right) \cdot \mathbf{r}}, \quad v_{j} \sim e^{i\left(\mathbf{k}-\mathbf{k}_{j}\right) \cdot \mathbf{r}},
$$

which is required for $\varphi_{j}(\mathbf{r}, t)$ to have the same Galilean transformation as $\varphi_{j}(\mathbf{r})$. Then it follows that

$$
\begin{aligned}
& \left(\hat{H}_{j}(\varphi)-E_{j}\right) u_{j}=\left(K_{j}+\hbar \mathbf{k} \cdot \mathbf{V}_{j}\right) u_{j} \\
& \left(\hat{H}_{j}(\varphi)-E_{j}\right) v_{j}=\left(K_{j}-\hbar \mathbf{k} \cdot \mathbf{V}_{j}\right) v_{j} .
\end{aligned}
$$

Therefore, Eqs. (14.17) retain practically the same form, but with the frequency $\omega$ shifted as

$$
\omega \rightarrow \omega-\varepsilon_{j}, \quad \varepsilon_{j} \equiv \mathbf{k} \cdot \mathbf{V}_{j}
$$

which corresponds to the Doppler shift.

Consider now a binary mixture. Without loss of generality, we may connect the system of coordinates with one of the components, say with the first one, so that $\mathbf{V}_{1}=0$. Then $\mathbf{V}_{2} \equiv \mathbf{v}$ is the velocity of the second component with respect to the first. The dynamical matrix $\hat{D}=\left[D_{i j}\right]$ in the spectral equation (14.21) has the same elements except

$$
D_{22}=-D_{11}+\hbar \mathbf{k} \cdot \mathbf{v}, \quad D_{44}=-D_{22}+\hbar \mathbf{k} \cdot \mathbf{v} .
$$

In general, instead of Eq. (14.26), we now have

$$
\left[\left(\omega-\varepsilon_{1}\right)^{2}-\omega_{1}^{2}\right]\left[\left(\omega-\varepsilon_{2}\right)^{2}-\omega_{2}^{2}\right]=\omega_{12}^{4} .
$$

In the chosen system of coordinates, connected with the first component, Eq. (14.48) simplifies to

$$
\left(\omega-\omega_{1}^{2}\right)\left[(\omega-\varepsilon)^{2}-\omega_{2}^{2}\right]=\omega_{12}^{4},
$$

where

$$
\varepsilon \equiv \mathbf{k} \cdot \mathbf{v}=k v \cos \vartheta
$$


The spectral equation (14.49) is the fourth-order algebraic equation

$$
\omega^{4}-2 \varepsilon \omega^{3}-\left(\omega_{1}^{2}+\omega_{2}^{2}-\varepsilon^{2}\right) \omega^{2}+2 \varepsilon \omega_{1}^{2} \omega+\omega_{1}^{2} \omega_{2}^{2}-\omega_{12}^{4}-\varepsilon^{2} \omega_{1}^{2}=0 .
$$

As we know, this equation can define not more than two stable, that is positive, solutions for the spectrum $\omega_{ \pm}(k)$. By Descartes' theorem, the necessary condition for Eq. (14.51) to possess two positive solutions for arbitrary $\varepsilon \in[-k v,+k v]$ is

$$
\omega_{1}^{2} \omega_{2}^{2}>\omega_{12}^{4}+\varepsilon^{2} \omega_{1}^{2} .
$$

Since this inequality is to be true for all $\vartheta \in[0, \pi]$, we can put here the maximal $\varepsilon=k v$. In this way, we obtain

$$
\omega_{1}^{2} \omega_{2}^{2}>\omega_{12}^{4}+\left(\omega_{1} k v\right)^{2} .
$$

In the long-wave limit, $k \rightarrow 0$, this gives

$$
c_{1}^{2} c_{2}^{2}>c_{12}^{4}+c_{1}^{2} v^{2} .
$$

Even if the immovable components do mix, they stratify as soon as the relative velocity reaches the critical value

$$
v_{c}=\frac{\sqrt{c_{1}^{2} c_{2}^{2}-c_{12}^{4}}}{c_{1}} .
$$

The stratification appears first inside the cone of the angle

$$
\vartheta_{c}=\arccos \frac{v_{c}}{v}
$$

With the notations 14.23$)$ and (14.25), the condition (14.54) becomes

$$
A_{11} A_{22}>A_{12}^{2}+\frac{m_{2} v^{2}}{\rho_{2}} A_{11}
$$

while for the critical velocity (14.55) one gets

$$
v_{c}^{2}=\frac{\rho_{2}}{m_{2} A_{11}}\left(A_{11} A_{22}-A_{12}^{2}\right) .
$$

Invoking the relation (14.12), we find the stability condition

$$
\frac{a_{11} a_{22}}{a_{12}^{2}}>\frac{\left(m_{1}+m_{2}\right)^{2}}{4 m_{1} m_{2}}+\frac{m_{2}^{2} v^{2} a_{11}}{4 \pi \hbar^{2} \rho_{2} a_{12}^{2}}
$$

expressed through the atomic scattering lengths. And the squared critical velocity (14.58) takes the form

$$
v_{c}^{2}=\frac{4 \pi \hbar^{2} \rho_{2} a_{12}^{2}}{m_{2}^{2} a_{11}}\left[\frac{a_{11} a_{22}}{a_{12}^{2}}-\frac{\left(m_{1}+m_{2}\right)^{2}}{4 m_{1} m_{2}}\right] .
$$

The value of the critical velocity (14.60) depends on the parameters of the species involved. For instance, in the case of alkali atoms, $m_{2} / \hbar \sim 10^{5} \mathrm{~s} / \mathrm{cm}^{2}, a_{11} \sim a_{12} \approx 5.5 \times 10^{-7} \mathrm{~cm}$, from where

$$
\frac{4 \pi \hbar^{2} a_{12}^{2}}{m_{2}^{2}} \sim 10^{-15} \frac{\mathrm{cm}^{5}}{\mathrm{~s}^{2}}
$$

Typical atomic-trap condensate densities are $\rho_{2} \sim 10^{12}-10^{15} \mathrm{~cm}^{-3}$. But what is needed first of all in order to have a finite critical velocity is that immovable components could be mixed, which requires the positiveness of the expression in the square bracket of Eq. (14.60). 


\subsection{Mixing by Feshbach Resonance}

By definition, Feshbach resonances involve intermediate states that are quasi-bound [396]. These intermediate states are not bound in the true sense, as they acquire a finite lifetime due to the interaction with continuum states of other scattering channels. The metastable objects, formed in the process of the Feshbach resonance atom-atom scattering, are molecules with electronic and nuclear spins that have been rearranged from the spins of the colliding atoms by virtue of the hyperfine interaction. It is important that the difference of the initial and intermediate state energies can be varied by means of an external magnetic field. The effective scattering length that describes the low-energy binary collisions similarly varies with the near-resonant magnetic field. Thus, employing the Feshbach resonances, it is possible to create two-component mixtures consisting of atoms and of molecules formed by these atoms. Since the overlapping components can be either stable or unstable with respect to stratification, depending on the relation between their scattering lengths, one could render the components miscible or immiscible by varying their scattering lengths. The Feshbach resonances were recently observed in ultra-cold atomic gases of ${ }^{87} \mathrm{Rb}\left[12\right.$ and ${ }^{23} \mathrm{Na}$ [11]. An important feature of the experiment [11] is that the Feshbach resonances were observed in an atomic Bose-condensed system. In this way, it looks feasible to create a two-component system of Bose-condensed atoms and molecules, with rather rich properties and with a variety of applications [26].

When two atoms of mass $m_{1}$ each form a Feshbach quasi-molecule, the mass of the latter is

$$
m_{2} \cong 2 m_{1} \text {. }
$$

Starting with a total number of atoms in a trap, $N$, one can form, via the magnetically controlled Feshbach resonance, $N_{2}$ molecules coexisting with $N_{1}$ unbound atoms. Then, between the number of unbound atoms and that of molecules, there is the relation

$$
N_{1}+2 N_{2}=N \text {. }
$$

This conservation law for the total number of atoms imposes the relation

$$
\mu_{2}=2 \mu_{1}
$$

between the chemical potentials of molecules, $\mu_{2}$, and of unbound atoms, $\mu_{1}$.

The Hamiltonian of an atomic-molecular mixture can be presented as

$$
\begin{gathered}
H=\sum_{i=1}^{2} \int \psi_{i}^{\dagger}(\mathbf{r})\left(-\frac{\hbar^{2} \nabla^{2}}{2 m_{i}}-\mu_{i}+U_{i}\right) \psi_{i}(\mathbf{r}) d \mathbf{r}+ \\
+\frac{1}{2} \sum_{i j}^{2} \int \psi_{i}^{\dagger}(\mathbf{r}) \psi_{j}^{\dagger}\left(\mathbf{r}^{\prime}\right) \Phi_{i j}\left(\mathbf{r}-\mathbf{r}^{\prime}\right) \psi_{j}\left(\mathbf{r}^{\prime}\right) \psi_{i}(\mathbf{r}) d \mathbf{r} d \mathbf{r}^{\prime}+ \\
+\frac{1}{2} \int \psi_{1}^{\dagger}(\mathbf{r}) \psi_{1}^{\dagger}(\mathbf{r}) \Theta_{12}\left(\mathbf{r}-\mathbf{r}^{\prime}\right) \psi_{2}\left(\mathbf{r}^{\prime}\right) d \mathbf{r} d \mathbf{r}^{\prime}+ \\
+\frac{1}{2} \int \psi_{2}^{\dagger}(\mathbf{r}) \Theta_{21}\left(\mathbf{r}-\mathbf{r}^{\prime}\right) \psi_{1}\left(\mathbf{r}^{\prime}\right) \psi_{1}\left(\mathbf{r}^{\prime}\right) d \mathbf{r} d \mathbf{r}^{\prime},
\end{gathered}
$$

where the last two terms describe the atom-molecule reaction with a transition amplitude having the symmetry properties

$$
\Theta_{12}(\mathbf{r})=\Theta_{12}(-\mathbf{r})=\Theta_{21}(\mathbf{r}) .
$$

Note that the global gauge symmetry, connected with the transformation $\psi_{j} \rightarrow e^{i \alpha} \psi_{j}$ is broken for the Hamiltonian (14.64). However, it possesses the gauge symmetry with respect to the transformation

$$
\psi_{j} \rightarrow e^{i \alpha_{j}} \psi_{j} \quad\left(2 \alpha_{1}=\alpha_{2}\right)
$$

which is related to the atom-number conservation law (14.62). The anomalous averages

$$
<\psi_{j}>=<\psi_{i} \psi_{j}>=0, \quad<\psi_{1} \psi_{1} \psi_{2}>=<\psi_{2} \psi_{2} \psi_{1}>=0,
$$


and alike, which are not invariant with respect to the gauge transformation (14.66), are zero. But the averages like

$$
<\psi_{1}^{\dagger} \psi_{1}^{\dagger} \psi_{2}>\neq 0
$$

that are invariant with respect to the transformation (14.66), are not zero.

The Heisenberg equations for the atomic, $\psi_{1}$, and molecular, $\psi_{2}$, field operators can be written as

$$
\begin{gathered}
i \hbar \frac{\partial}{\partial t} \psi_{1}(\mathbf{r}, t)=H_{1}(\psi) \psi_{1}(\mathbf{r}, t)+\psi_{1}^{\dagger}(\mathbf{r}, t) \int \Theta_{12}\left(\mathbf{r}-\mathbf{r}^{\prime}\right) \psi_{2}\left(\mathbf{r}^{\prime}, t\right) d \mathbf{r}^{\prime}, \\
i \hbar \frac{\partial}{\partial t} \psi_{2}(\mathbf{r}, t)=H_{2}(\psi) \psi_{2}(\mathbf{r}, t)+\frac{1}{2} \int \Theta_{12}\left(\mathbf{r}-\mathbf{r}^{\prime}\right) \psi_{1}\left(\mathbf{r}^{\prime}, t\right) \psi_{1}\left(\mathbf{r}^{\prime}, t\right) d \mathbf{r}^{\prime},
\end{gathered}
$$

where the notation

$$
H_{i}(\psi) \equiv-\frac{\hbar^{2} \nabla^{2}}{2 m_{i}}-\mu_{i}+U_{i}(\mathbf{r}, t)+\sum_{j} \int \Phi_{i j}\left(\mathbf{r}-\mathbf{r}^{\prime}\right) \psi_{j}^{\dagger}\left(\mathbf{r}^{\prime}, t\right) \psi_{j}\left(\mathbf{r}^{\prime}, t\right) d \mathbf{r}^{\prime}
$$

is used.

Assuming dilute gases, one models the interaction potentials and transition amplitudes by local functions

$$
\Phi_{i j}(\mathbf{r})=A_{i j} \delta(\mathbf{r}), \quad \Theta_{12}(\mathbf{r})=B_{12} \delta(\mathbf{r}) .
$$

Supposing that the whole mixture is in a coherent state, one has for the corresponding coherent fields

$$
\begin{gathered}
i \hbar \frac{\partial}{\partial t} \varphi_{1}=\left[\hat{H}_{1}(\varphi)-\mu_{1}\right] \varphi_{1}+\sqrt{N_{2}} B_{12} \varphi_{1}^{*} \varphi_{2}, \\
i \hbar \frac{\partial}{\partial t} \varphi_{2}=\left[\hat{H}_{2}(\varphi)-\mu_{2}\right] \varphi_{2}+\frac{N_{1}}{2 \sqrt{N_{2}}} B_{12} \varphi_{1}^{2}
\end{gathered}
$$

where $\rho_{j} \equiv N_{j} / V$ and

$$
\hat{H}_{i}(\varphi)=-\frac{\hbar^{2} \nabla^{2}}{2 m_{i}}+U_{i}(\mathbf{r}, t)+\sum_{j=1}^{2} N_{j} A_{i j}\left|\varphi_{j}\right|^{2} .
$$

Looking for stationary solutions of Eqs. (14.72) in the standard form (14.14), we see that the related energies $E_{j}$ are connected with each other by the relation

$$
E_{2}=2 E_{1} .
$$

Introducing the notation

$$
E \equiv E_{1}+\mu_{1},
$$

we obtain the following equations for the stationary coherent fields:

$$
\begin{gathered}
\hat{H}_{1}(\varphi) \varphi_{1}(\mathbf{r})+\sqrt{N_{2}} B_{12} \varphi_{1}^{*}(\mathbf{r}) \varphi_{2}(\mathbf{r})=E \varphi_{1}(\mathbf{r}) \\
\hat{H}_{2}(\varphi) \varphi_{2}(\mathbf{r})+\frac{N_{1}}{2 \sqrt{N_{2}}} B_{12} \varphi_{1}^{2}(\mathbf{r})=2 E \varphi_{2}(\mathbf{r})
\end{gathered}
$$

Wanting to study collective excitations in this reacting mixture, we may linearize Eqs. (14.72) after substituting there the form (14.16). This results in the system of four equations

$$
\begin{aligned}
& \left(\hbar \omega-\hat{H}_{1}(\varphi)+E\right) u_{1}-\sqrt{N_{2}} B_{12}\left(\varphi_{1}^{*} u_{2}+\varphi_{2} v_{1}\right)-\sum_{i=1}^{2} N_{i} A_{i 1}\left(\varphi_{i}^{*} u_{i}+\varphi_{i} v_{i}\right) \varphi_{1}=0, \\
& \left(\hbar \omega+\hat{H}_{1}(\varphi)-E\right) v_{1}+\sqrt{N_{2}} B_{12}\left(\varphi_{1} v_{2}+\varphi_{2}^{*} u_{1}\right)+\sum_{i=1}^{2} N_{i} A_{i 1}\left(\varphi_{i}^{*} u_{i}+\varphi_{i} v_{i}\right) \varphi_{1}^{*}=0
\end{aligned}
$$




$$
\begin{aligned}
& \left(\hbar \omega-\hat{H}_{2}(\varphi)+2 E\right) u_{2}-\frac{N_{1}}{\sqrt{N_{2}}} B_{12} \varphi_{1} u_{1}-\sum_{i=1}^{2} N_{i} A_{i 2}\left(\varphi_{i}^{*} u_{i}+\varphi_{i} v_{i}\right) \varphi_{2}=0 \\
& \left(\hbar \omega+\hat{H}_{2}(\varphi)-2 E\right) v_{2}+\frac{N_{1}}{\sqrt{N_{2}}} B_{12} \varphi_{1}^{*} v_{1}+\sum_{i=1}^{2} N_{i} A_{i 2}\left(\varphi_{i}^{*} u_{i}+\varphi_{i} v_{i}\right) \varphi_{2}^{*}=0
\end{aligned}
$$

where $\hat{H}_{i}(\varphi)$ are defined for the stationary states $\varphi_{i}(\mathbf{r})$.

In the uniform approximation (14.18), the stationary equations (14.76) yield

$$
\begin{gathered}
E=U_{1}+\rho_{1} A_{11}+\rho_{2} A_{12}+\sqrt{\rho_{2}} B_{12}, \\
2 E=U_{2}+\rho_{1} A_{12}+\rho_{2} A_{22}+\frac{\rho_{1}}{2 \sqrt{\rho_{2}}} B_{12} .
\end{gathered}
$$

Under given values for $A_{11}, A_{12}, A_{22}$, and $B_{12}$, Eq. (14.78) defines the relation between the atomic density $\rho_{1}$ and the molecular density $\rho_{2}$. Since $\rho_{i} \equiv n_{i} N_{i}$, this means that the number of unbound atoms $N_{1}$ and that of molecules $N_{2}$ are not arbitrary but are related with each other through Eqs. (14.78).

Keeping in mind the uniform approximation, when $n_{i}=1 / V$, and looking for the solutions of Eqs. (14.77) in the form of plane waves $e^{i \mathbf{k} \cdot \mathbf{r}}$, we come to the equations

$$
\begin{gathered}
\left(\hbar \omega-K_{1}\right) u_{1}-\sqrt{\rho_{2}} B_{12}\left(u_{2}+v_{1}\right)-\sum_{i=1}^{2} \rho_{i} A_{i 1}\left(u_{i}+v_{i}\right)=0, \\
\left(\hbar \omega-K_{2}\right) u_{2}-\frac{\rho_{1}}{\sqrt{\rho_{2}}} B_{12} u_{1}-\sum_{i=1}^{2} \rho_{i} A_{i 2}\left(u_{i}+v_{i}\right)=0, \\
\left(\hbar \omega+K_{1}\right) v_{1}+\sqrt{\rho_{2}} B_{12}\left(v_{2}+u_{1}\right)+\sum_{i=1}^{2} \rho_{i} A_{i 1}\left(u_{i}+v_{i}\right)=0, \\
\left(\hbar \omega+K_{2}\right) v_{2}+\frac{\rho_{1}}{\sqrt{\rho_{2}}} B_{12} v_{1}+\sum_{i=1}^{2} \rho_{i} A_{i 2}\left(u_{i}+v_{i}\right)=0 .
\end{gathered}
$$

The spectral equation can be presented as in Eq. (14.21), but with the dynamic matrix having the elements

$$
\begin{gathered}
D_{11}=K_{1}+\rho_{1} A_{11}, \quad D_{12}=\rho_{2} A_{12}+\sqrt{\rho_{2}} B_{12}, \quad D_{13}=\rho_{1} A_{11}+\sqrt{\rho_{2}} B_{12}, \quad D_{14}=\rho_{2} A_{12}, \\
D_{21}=\rho_{1} A_{12}+\frac{\rho_{1}}{\sqrt{\rho_{2}}} B_{12}, \quad D_{22}=K_{2}+\rho_{2} A_{22}, \quad D_{23}=\rho_{1} A_{12}, \quad D_{24}=\rho_{2} A_{22}, \\
D_{31}=-D_{13}, \quad D_{32}=-D_{14}, \quad D_{33}=-D_{11}, \quad D_{34}=-D_{12}, \\
D_{41}=-D_{23}, \quad D_{42}=-D_{24}, \quad D_{43}=-D_{21}, \quad D_{44}=-D_{22} .
\end{gathered}
$$

The general form of the spectral equation is rather cumbersome, so we shall not write it down. Some particular cases have been studied in Ref. [26].

Similarly to Sec. 14.3, we can study the stability conditions for the atom-molecule mixture. For example, to find the condition of dynamic stability, we need to find the inequality guaranteeing the positiveness of the collective excitation spectrum corresponding to the oscillation of components with respect to each other. To derive the condition of thermodynamic stability, we have to compare the coherent average of the mixture Hamiltonian (14.64), which in the uniform approximation is

$$
<H>_{N}=N_{1}\left(U_{1}-\mu_{1}\right)+N_{2}\left(U_{2}-\mu_{2}\right)+\frac{N_{1}^{2} A_{11}+2 N_{1} N_{2} A_{12}+N_{2}^{2} A_{22}}{2\left(V_{1}+V_{2}\right)}+N_{1} \sqrt{\rho_{2}} B_{12},
$$


with the energy (14.41) of a stratified system, where $U_{i}$ is to be replaced by $U_{i}-\mu_{i}$. Then the condition of thermodynamic stability (14.42) becomes

$$
N_{1}^{2} V_{2}^{2} A_{11}+N_{2}^{2} V_{1}^{2} A_{22}-2 N_{1} N_{2} V_{1} V_{2} A_{12}-2 N_{1} \sqrt{N_{2} V} V_{1} V_{2} B_{12}>0 .
$$

The sufficient stability conditions for each component separately are $A_{11}>0$ and $A_{22}>0$. If so, the condition of thermodynamic stability of the mixture reads

$$
\left(N_{1} V_{2} \sqrt{A_{11}}-N_{2} V_{1} \sqrt{A_{22}}\right)^{2}+2 N_{1} N_{2} V_{1} V_{2}\left(\sqrt{A_{11} A_{22}}-A_{12}\right)-2 N_{1} \sqrt{N_{2} V} V_{1} V_{2} B_{12}>0 .
$$

From here, the sufficient condition of thermodynamic stability is

$$
A_{11} A_{22}>\left(A_{12}+\frac{B_{12}}{\sqrt{\rho_{2}}}\right)^{2}
$$

where $\rho_{2} \equiv N_{2} / V$. Since the low-energy Feshbach resonances make it feasible to vary the effective scattering lengths by a near-resonant external magnetic field, one could realize different experiments with stable mixtures as well as with stratifying components. 


\section{Chapter 15}

\section{Topological Coherent Modes}

Coherent modes are defined in Chapter 8 as stationary solutions of the Gross-Pitaevskii equation. The groundstate coherent mode, with a single-particle energy $E_{0}$, corresponds to the Bose-Einstein condensate. In an equilibrium statistical system, the Bose-condensed state is always the ground single-particle state.

An intriguing question is whether one could create non-groundstate condensates of Bose atoms, that is, a macroscopic occupation of a non-ground single-particle state. Clearly, if this is possible, this could be done only in a non-equilibrium system. Second, in order to transfer atoms from a single-particle ground state, with an energy $E_{0}$, to another state of higher energy $E_{j}$, one should subject the system to the action of a resonance field with a frequency close to the transition frequency $\left(E_{j}-E_{0}\right) / \hbar$. Hence, this is to be a resonance process.

The possibility of the resonance formation of non-groundstate condensates of Bose atoms was first advanced in Ref. [139], these condensates being associated with excited coherent modes. Such nonlinear coherent modes have also been considered recently in Refs. [140, 397, 398]. One often terms these excited coherent modes as topological in order to stress their distinction from elementary collective excitations. The latter correspond to small linear oscillations around a state, thence these small oscillations do not change the macroscopic density distribution in space. But different coherent modes have principally different space dependence, because of which they are termed topological.

\subsection{Resonance Field Modulation}

The Gross-Pitaevskii equation, describing a coherent field $\varphi=\varphi(\mathbf{r}, t)$, is

$$
i \hbar \frac{\partial \varphi}{\partial t}=\left[\hat{H}(\varphi)+\hat{V}_{\text {res }}\right] \varphi
$$

where, in addition to the nonlinear Hamiltonian

$$
\hat{H}(\varphi)=-\frac{\hbar^{2}}{2 m_{0}} \nabla^{2}+U(\mathbf{r})+N A|\varphi|^{2},
$$

we include a resonant field

$$
\hat{V}_{\text {res }}=V_{1}(\mathbf{r}) \cos \omega t+V_{2}(\mathbf{r}) \sin \omega t .
$$

We assume that at the initial time the system is Bose-condensed to the ground state

$$
\varphi(\mathbf{r}, 0)=\varphi_{0}(\mathbf{r})
$$

characterized by the energy $E_{0}$.

The transition frequencies between coherent modes are given by the equation

$$
\hbar \omega_{m n} \equiv E_{m}-E_{n}
$$


in which the spectrum of coherent modes is defined by the eigenproblem

$$
\hat{H}\left(\varphi_{n}\right) \varphi_{n}=E_{n} \varphi_{n} .
$$

Suppose that our aim is to transfer atoms from the ground state $\varphi_{0}$ to a chosen state $\varphi_{j}$. Therefore, we require that the frequency of the modulating field (15.3) be close to the transition frequency

$$
\omega_{j} \equiv \frac{E_{j}-E_{0}}{\hbar} .
$$

The closeness implies the quasiresonance condition

$$
\left|\frac{\Delta \omega}{\omega}\right| \ll 1, \quad \Delta \omega \equiv \omega-\omega_{j} .
$$

Another important requirement is that the spectrum of coherent modes be not equidistant 139. In fact, if that were the case, then the pumping of atoms from the ground state to the chosen particular state would, at the same time, induce transitions from the latter to another equidistant state and from the latter to even higher equidistant states. Thus, all atoms would be dispersed over all states making it impossible to achieve a macroscopic population of one of them. Fortunately, as is shown in Chapters 10 and 11, the spectrum of coherent modes is not equidistant because of the nonlinearity induced by atomic interactions.

Let us look for the solution of Eq. (15.1) in the form of the sum

$$
\varphi(\mathbf{r}, t)=\sum_{n} c_{n}(t) \varphi_{n}(\mathbf{r}, t)
$$

over the coherent modes

$$
\varphi_{n}(\mathbf{r}, t)=\varphi_{n}(\mathbf{r}) \exp \left(-\frac{i}{\hbar} E_{n} t\right) .
$$

It is worth noting that the presentation (15.8) does not require the set $\left\{\varphi_{n}(\mathbf{r})\right\}$ to form a complete basis. As can be checked in any textbook on Quantum Mechanics or Functional Analysis, the property of completeness of a basis presupposes that an arbitrary function from the considered Hilbert space could be presented as an expansion over this basis. We do not require here such a restrictive property for all functions, but we invoke just the sole expansion, looking for a solution in the form of (15.8).

What we need in the following is the assumption that the coefficients $c_{n}(t)$ in the sum (15.8) vary in time slower than the exponentials in $\varphi_{n}(\mathbf{r}, t)$, that is,

$$
\left|\frac{d c_{n}}{d t}\right| \ll E_{n} .
$$

Looking for a solution in the form (15.8), one has to substitute it into Eq. (15.1). To obtain equations for the coefficients $c_{n}(t)$, one may invoke the averaging techniques [399]. This is possible because, according to the inequality (15.9), the functions $c_{n}(t)$ can be classified as slow, compared to the fast functions $\exp \left(-i E_{n} t / \hbar\right)$. Thus, $c_{n}(t)$ can be treated as quasi-invariants. Multiplying Eq. (15.1) by $\varphi_{n}^{*}(\mathbf{r}, t)$ and integrating the result over $\mathbf{r}$ and averaging over time as

$$
\lim _{\tau \rightarrow \infty} \frac{1}{\tau} \int_{0}^{\tau} F(t) d t
$$

keeping $c_{n}$ as quasi-invariants, one obtains an equation describing the guiding centers for $c_{n}(t)$. Averaging over time, one uses the equalities

$$
\begin{gathered}
\lim _{\tau \rightarrow \infty} \frac{1}{\tau} \int_{0}^{\tau} \exp \left\{\frac{i}{\hbar}\left(E_{m}-E_{n}\right) t\right\} d t=\delta_{m n}, \\
\lim _{\tau \rightarrow \infty} \frac{1}{\tau} \int_{0}^{\tau} \exp \left\{\frac{i}{\hbar}\left(E_{m}+E_{k}-E_{n}-E_{l}\right) t\right\} d t=\delta_{m n} \delta_{k l}+\delta_{m l} \delta_{n k}-\delta_{m k} \delta_{k n} \delta_{n l} .
\end{gathered}
$$


The latter assume that the spectrum $E_{n}$ is nondegenerate. Generally, in the presence of the nonlinear term in the Hamiltonian (15.2), this is true. But even if the spectrum $E_{n}$ were degenerate, one could avoid complications in the following way. One may add to the Hamiltonian (15.2) a term lifting the degeneracy and to set this term to zero at the end of the calculations.

Note that normalizing the function $(15.8)$ as $(\varphi, \varphi)=1$, one gets

$$
\sum_{m n} c_{m}^{*}(t) c_{n}(t)\left(\varphi_{m}, \varphi_{n}\right) e^{-i \omega_{m n} t}=1
$$

Averaging this over time, according to the rule (15.10) and invoking condition (15.9), gives

$$
\sum_{n}\left|c_{n}(t)\right|^{2}=1
$$

From here a useful relation follows:

$$
\left|c_{n}(t)\right|^{2}=1-\sum_{m(\neq n)}\left|c_{m}(t)\right|^{2} .
$$

Substituting the form (15.8) into Eq. (15.1) and accomplishing the described time-averaging procedure results in the equation

$$
\frac{d c_{n}}{d t}=-i \sum_{m(\neq n)} \alpha_{n m}\left|c_{m}\right|^{2} c_{n}-\frac{i}{2} \delta_{n 0} \beta c_{j} e^{i \Delta \omega t}-\frac{i}{2} \delta_{n j} \beta^{*} c_{0} e^{-i \Delta \omega t},
$$

in which the amplitude

$$
\alpha_{n m} \equiv A \frac{N}{\hbar} \int\left|\varphi_{n}(\mathbf{r})\right|^{2}\left(2\left|\varphi_{m}(\mathbf{r})\right|^{2}-\left|\varphi_{n}(\mathbf{r})\right|^{2}\right) d \mathbf{r}
$$

is due to the nonlinear term in the Hamiltonian (15.2), while the transition amplitude

$$
\beta \equiv \frac{1}{\hbar} \int \varphi_{0}^{*}(\mathbf{r}) V(\mathbf{r}) \varphi_{j}(\mathbf{r}) d \mathbf{r}, \quad V(\mathbf{r}) \equiv V_{1}(\mathbf{r})-i V_{2}(\mathbf{r}),
$$

is related to the resonant modulating field $(15.3)$. In the process of the time averaging $(15.10)$, the function $\exp (i \Delta \omega t)$ is also treated as slow because of the quasiresonance condition (15.7).

Equation (15.12) shows that the resonant field induces transitions only between the ground-state and a chosen $j$-level. At first glance, the nonlinear term, being nonresonant, could induce transitions between all levels, changing the corresponding fractional populations

$$
n_{m}(t) \equiv\left|c_{m}(t)\right|^{2} .
$$

However, from Eq. (15.12) it follows that for all levels $m \neq 0, j$, except the two selected resonant levels, one has

$$
\frac{d}{d t} n_{m}(t)=0 \quad(m \neq 0, j) .
$$

This, with the initial condition $n_{m}(0)=0$, yields

$$
n_{m}(t)=0 \quad(m \neq 0, j) .
$$

Similarly, $c_{m}(t)=0$ for all $m \neq 0, j$. Therefore, Eq. (15.12) is equivalent to the system of two equations

$$
\begin{gathered}
\frac{d c_{0}}{d t}=-i \alpha_{0 j} n_{j} c_{0}-\frac{i}{2} \beta c_{j} e^{i \Delta \omega t}, \\
\frac{d c_{j}}{d t}=-i \alpha_{j 0} n_{0} c_{j}-\frac{i}{2} \beta^{*} c_{0} e^{-i \Delta \omega t} .
\end{gathered}
$$


The initial conditions to these equations, according to Eq. (15.4), are

$$
c_{0}(0)=1, \quad c_{j}(0)=0 .
$$

The equations for the fractional populations (15.15) immediately follow from Eqs. (15.16) giving

$$
\frac{d n_{0}}{d t}=\operatorname{Im}\left(\beta e^{i \Delta \omega t} c_{0}^{*} c_{j}\right), \quad \frac{d n_{j}}{d t}=\operatorname{Im}\left(\beta^{*} e^{-i \Delta \omega t} c_{j}^{*} c_{0}\right),
$$

with the corresponding initial conditions

$$
n_{0}(0)=1, \quad n_{j}(0)=0,
$$

resulting from the conditions (15.17). The normalization (15.11) for the fractional populations reduces to the equation

$$
n_{0}(t)+n_{j}(t)=1
$$

In what follows, for the simplicity of notation, we write

$$
\alpha \equiv \alpha_{0 j}
$$

and set $\alpha_{0 j}=\alpha_{j 0}$.

Note that in deriving Eqs. (15.16), the orthogonality of the coherent modes $\varphi_{m}(\mathbf{r})$ and $\varphi_{n}(\mathbf{r})$, for $m \neq n$, has not been assumed. What is used is the condition (15.9) permitting one to invoke the averaging technique [399]. In addition, employing for these modes the solutions of Chapter 10, one can check that $\left|\left(\varphi_{m}, \varphi_{n}\right)\right|$ are less or of order 0.1 if $m \neq n$. Hence the coherent modes can be treated as approximately orthogonal since $\left|\left(\varphi_{m}, \varphi_{n}\right)\right| \ll 1$ for $m \neq n$.

The solutions to Eqs. (15.16) and (15.18) can be obtained analytically, provided the inequality

$$
\left|\frac{\beta}{\alpha}\right| \ll 1
$$

holds true. In that case, one can again resort to the averaging technique [399], being based on the fact that the functions $c_{0}(t)$ and $c_{j}(t)$ can be classified as fast, compared to the slow functions $n_{0}(t)$ and $n_{j}(t)$. With the slow functions treated as quasi-invariants, Eqs. (15.15) are linear equations with respect to $c_{0}(t)$ and $c_{j}(t)$, which gives

$$
\begin{gathered}
c_{0}=\left[\cos \frac{\Omega t}{2}+i \frac{\alpha\left(n_{0}-n_{j}\right)-\Delta \omega}{\Omega} \sin \frac{\Omega t}{2}\right] \exp \left\{-\frac{i}{2}(\alpha-\Delta \omega) t\right\}, \\
c_{j}=-i \frac{\beta^{*}}{\Omega} \sin \frac{\Omega t}{2} \exp \left\{-\frac{i}{2}(\alpha+\Delta \omega) t\right\},
\end{gathered}
$$

where the collective frequency $\Omega$, defined by the equality

$$
\Omega^{2} \equiv\left[\alpha\left(n_{0}-n_{j}\right)-\Delta \omega\right]^{2}+|\beta|^{2},
$$

is introduced. Comparing our case with the resonant excitation of atoms in optics [296], we see that $|\beta|$ plays the role of the Rabi frequency, while $\sqrt{|\beta|^{2}+(\Delta \omega)^{2}}$ is what is called the effective Rabi frequency. The quantity $\Omega$, defined in Eq. (15.23), differs from the effective Rabi frequency by the presence of the term containing the interaction amplitude $\alpha$. Because of this, in our case, $\Omega$ could be called the collective Rabi frequency, although it is not a parameter but a function depending on time through the fractional populations $n_{0}(t)$ and $n_{j}(t)$. For the latter, we get

$$
n_{0}=1-\frac{|\beta|^{2}}{\Omega^{2}} \sin ^{2} \frac{\Omega t}{2}, \quad n_{j}=\frac{|\beta|^{2}}{\Omega^{2}} \sin ^{2} \frac{\Omega t}{2} .
$$

If at some finite time $t_{0}$, the modulation field (15.3) is switched off, then, as follows from Eqs. (15.18), the fractional populations stand constant, with the values $n_{0}\left(t_{0}\right)$ and $n_{j}\left(t_{0}\right)$. Then, we have a mixture of two 
topological modes. This mixture will, of course, not exist for ever, but during the lifetime of the corresponding modes limited by atomic collisions. For instance, the loss rates caused by binary depolarizing collisions can be estimated as

$$
\begin{aligned}
& \gamma_{0}=\lambda_{00} N^{2} n_{0}^{2} \int\left|\varphi_{0}(\mathbf{r})\right|^{4} d \mathbf{r}+\lambda_{0 j} N^{2} n_{0} n_{j} \int\left|\varphi_{0}(\mathbf{r})\right|^{2}\left|\varphi_{j}(\mathbf{r})\right|^{2} d \mathbf{r}, \\
& \gamma_{j}=\lambda_{j j} N^{2} n_{j}^{2} \int\left|\varphi_{j}(\mathbf{r})\right|^{4} d \mathbf{r}+\lambda_{j 0} N^{2} n_{j} n_{0} \int\left|\varphi_{j}(\mathbf{r})\right|^{2}\left|\varphi_{0}(\mathbf{r})\right|^{2} d \mathbf{r},
\end{aligned}
$$

where $\lambda_{i j}$ are the related loss-rate coefficients and $n_{0}=n_{0}\left(t_{0}\right)$ and $n_{j}=n_{j}\left(t_{0}\right)$.

A modulating field (15.3) that is not monochromatic but characterized by a frequency distribution $\rho(\omega)$ that is centered at $\omega_{j}$ will cause heating of the system due to nonresonant transitions [400. The corresponding heating rate can be expressed as the sum

$$
\Gamma_{\text {heat }}=2 \pi \sum_{n(\neq j)}\left|\beta_{n 0}\right|^{2} \rho\left(\omega_{n 0}\right),
$$

in which

$$
\beta_{n 0} \equiv \frac{1}{\hbar} \int \varphi_{n}^{*}(\mathbf{r}) V(\mathbf{r}) \varphi_{0}(\mathbf{r}) d \mathbf{r} .
$$

If the density of frequencies $\rho(\omega)$ is sharply centered, say as $\rho(\omega) \approx \delta\left(\omega-\omega_{j 0}\right)$, then the heating rate is close to zero.

\subsection{Critical Dynamic Effects}

The solution (15.22) to the evolution equations $(15.16)$ has been obtained by using the averaging technique [399], which requires the inequality (15.21). Wanting to analyze the behavior of solutions to Eqs. (15.16) under an arbitrary relation between $\alpha$ and $\beta$, we have to solve these equations numerically. This behavior turned out to be surprisingly rich exhibiting unexpected critical effects 401.

For the numerical analysis of Eqs. 15.1), it is convenient to introduce the dimensionless parameters

$$
b \equiv \frac{|\beta|}{\alpha}, \quad \delta \equiv \frac{\Delta \omega}{\alpha}
$$

and to perform a scaling, measuring time in units of $\alpha^{-1}$. To return back to dimensional time, one has to make the substitution

$$
t \rightarrow \frac{t}{\alpha} .
$$

We solve [401] the system of equations (15.16) for different values of the parameters (15.26), keeping in mind that the dimensionless detuning is small,

$$
\delta \ll 1
$$

Varying the parameters (15.26), we find 401] that there exists a bifurcation line, described by the relation

$$
b+\delta \approx 0.5
$$

at which the qualitative behavior of solutions changes abruptly.

To illustrate the drastic change in the behavior of solutions, when crossing the bifurcation line $(15.29)$, let us first fix $b=0.4999$ and vary the detuning $\delta$. Figure 36 presents the fractional populations $n_{0}(t)$ and $n_{j}(t)$, defined as in Eq. (15.15), time being measured in units of $\alpha^{-1}$. In Fig. 36(a), the detuning is zero, $\delta=0$, and the behavior of the fractional populations approximately follows the law (15.24). Slightly changing the detuning to $\delta=0.0001$ essentially transforms the behavior to that in Fig. 36(b), where the top of $n_{j}(t)$ and the bottom of $n_{0}(t)$ become flat, touching each other, while the oscillation period is approximately doubled. Shifting the detuning by a tiny portion to $\delta=0.0001001$ results in Fig. 36(c), where the period is again doubled, and there appear the upward cusps of $n_{j}(t)$ and the downward cusps of $n_{0}(t)$. Increasing a little the detuning to 
$\delta=0.00011$ squeezes the oscillation period twice, as is shown in Fig. 36(d). Similar changes happen when crossing the bifurcation line under a fixed detuning and a varied transition amplitude, as illustrated in Fig. 37 .

The unusual behavior of the fractional populations is due to the nonlinearity of the evolution equations (15.16). It is known that systems of nonlinear differential equations can possess qualitatively different solutions for parameters differing by infinitesimally small values. The transfer from one type of solutions to another qualitatively different type is termed in the theory of dynamical systems bifurcation 402. At a bifurcation point, a dynamical system is structurally unstable. Bifurcations in dynamical systems are somewhat analogous to phase transitions and critical phenomena in equilibrium statistical systems [403. To elucidate this analogy for the present case, we have to consider the time-averaged properties of the system, which can be done as follows. First, it is necessary to define an effective Hamiltonian generating the evolution equations (15.16). This can be done by noticing that these equations can be presented in the Hamiltonian form

$$
i \frac{d c_{0}}{d t}=\frac{\partial H_{e f f}}{\partial c_{0}^{*}}, \quad i \frac{d c_{j}}{d t}=\frac{\partial H_{e f f}}{\partial c_{j}^{*}}
$$

with the effective Hamiltonian

$$
H_{e f f}=\alpha n_{0} n_{j}+\operatorname{Re}\left(\beta e^{i \Delta \omega t} c_{0}^{*} c_{j}\right) .
$$

Substituting here the approximate solutions $(15.22)$ yields

$$
H_{e f f}=\alpha n_{0} n_{j}-n_{j}\left[\alpha\left(n_{0}-n_{j}\right)-\Delta \omega\right] .
$$

The latter, with the normalization (15.20), gives

$$
H_{e f f}=\alpha n_{j}^{2}+n_{j} \Delta \omega .
$$

Averaging the fractional populations (15.24) over the explicit time and using this averaged quantity in the collective frequency (15.23), one has the averaged population

$$
\bar{n}_{j}=\frac{|\beta|^{2}}{2 \bar{\Omega}^{2}},
$$

in which the averaged collective frequency is given by the equality

$$
\bar{\Omega}^{2}=\left[\alpha\left(1-2 \bar{n}_{j}\right)-\Delta \omega\right]^{2}+|\beta|^{2} .
$$

The effective average energy can be defined by taking the effective Hamiltonian (15.32) with $n_{j}$ replaced by the averaged population (15.33), which gives

$$
E_{e f f}=\alpha \bar{n}_{j}^{2}+\bar{n}_{j} \Delta \omega .
$$

To study a kind of thermodynamics of the so defined effective system, it is possible to introduce the following characteristics. The pumping capacity

$$
C_{\beta} \equiv \frac{\partial E_{e f f}}{\partial|\beta|}
$$

describes the capacity of the system to store the energy pumped into it by the modulating field. The order parameter

$$
\eta \equiv \bar{n}_{0}-\bar{n}_{j}=1-2 \bar{n}_{j}
$$

characterizes the level of excitation, being $\eta=1$ for a system in the ground state and $\eta=-1$ for a completely excited system. The detuning susceptibility

$$
\chi_{\delta} \equiv\left|\frac{\partial \eta}{\partial \delta}\right|
$$

defines how a variation of detuning influences the order parameter. 
It is convenient to pass again to the dimensionless quantities (15.26) and to introduce the dimensionless average collective frequency

$$
\varepsilon \equiv \frac{\bar{\Omega}}{\alpha} .
$$

Then Eq. 15.34) takes the form

$$
\varepsilon^{4}\left(\varepsilon^{2}-b^{2}\right)=\left(\varepsilon^{2}-b^{2}-\varepsilon^{2} \delta\right)^{2} .
$$

The average energy $(15.35)$ becomes

$$
E_{e f f}=\frac{\alpha b^{2}}{2 \varepsilon}\left(\frac{b^{2}}{2 \varepsilon^{2}}+\delta\right) .
$$

And the order parameter (15.37) is

$$
\eta=1-\frac{b^{2}}{\varepsilon^{2}} .
$$

Taking into account the smallness of the detuning (15.28), one can simplify the above expressions.

Looking for a positive solution of Eq. (15.40), one can notice that there is the critical value

$$
b_{c}=\frac{1}{2}-\delta,
$$

at which the average collective frequency (15.39) has a jump, so that

$$
\varepsilon=\frac{1}{\sqrt{2}}\left[1-2 \delta+\sqrt{(1-2 \delta)^{2}-4 b^{2}}\right]^{1 / 2}
$$

for $0 \leq b \leq b_{c}$, but

$$
\varepsilon=b \quad\left(b>b_{c}\right) .
$$

The frequency (15.44) changes from

$$
\varepsilon=1-2 \delta \quad(b=0)
$$

to the critical frequency

$$
\varepsilon_{c}=\sqrt{\frac{1}{2}-\delta} \quad\left(b=b_{c}\right)
$$

with the jump at $b=b_{c}$ being

$$
\varepsilon\left(b_{c}+0\right)-\varepsilon\left(b_{c}-0\right)=\frac{1-\sqrt{2}}{2}-\left(1-\frac{1}{\sqrt{2}}\right) \delta .
$$

A sudden decrease in the frequency implies an abrupt increase in the oscillation period. The order parameter (15.42) varies from $\eta=1$ at $b=0$ to the critical value

$$
\eta_{c}=\frac{1}{2}+\delta \quad\left(b=b_{c}\right),
$$

becoming zero for $b>b_{c}$. The average energy (15.41), above $b_{c}$, does not depend on the value of $b$,

$$
E_{e f f}=\frac{\alpha}{2}\left(\frac{1}{2}+\delta\right) \quad\left(b>b_{c}\right) .
$$

Thus, the pumping capacity (15.36), order parameter (15.37), and detuning susceptibility (15.38) all are zero above $b_{c}$,

$$
C_{\beta}=0, \quad \eta=0, \quad \chi_{\delta}=0 \quad\left(b>b_{c}\right) .
$$

The behavior of these characteristics in the asymptotic vicinity of the critical line (15.43), below the critical pumping $b_{c}$, is as follows. With the notation

$$
\tau \equiv \frac{b_{c}-b}{b_{c}} \quad\left(b \leq b_{c}\right)
$$


the pumping capacity is

$$
C_{\beta} \simeq \frac{\sqrt{2}}{8} \tau^{-1 / 2}-\frac{1}{4}-\frac{3 \sqrt{2}}{32} \tau^{1 / 2}
$$

the order parameter becomes

$$
\eta \simeq \frac{1}{2}+\delta+\frac{\sqrt{2}}{2}(1-2 \delta) \tau^{1 / 2}
$$

and the detuning susceptibility takes the form

$$
\chi_{\delta} \simeq \frac{1}{\sqrt{2}} \tau^{-1 / 2}-1+\frac{\sqrt{2}}{8} \tau^{1 / 2} .
$$

This shows that the pumping capacity and detuning susceptibility diverge as $\tau \rightarrow 0$. Hence, Eq. (15.43) really defines a critical line where critical phenomena occur. The critical indices for all characteristics are $1 / 2$, satisfying the scaling relation

$$
\text { ind } C_{\beta}+2 \text { ind } \chi+\text { ind } \chi_{\delta}=2 \text {, }
$$

known for critical phenomena 403]; here ind is the abbreviation for index. The critical line (15.43) coincides with the bifurcation line (15.29).

\subsection{Spatio-Temporal Evolution of Density}

In the two-level picture of section 15.1 the coherent field (15.8) is

$$
\varphi(\mathbf{r}, t)=c_{0}(t) \varphi_{0}(\mathbf{r}) \exp \left(-\frac{i}{\hbar} E_{0} t\right)+c_{j}(t) \varphi_{j}(\mathbf{r}) \exp \left(-\frac{i}{\hbar} E_{j} t\right) .
$$

To study the spatio-temporal properties of an atomic cloud, it is convenient to average the density of atoms $N|\varphi(\mathbf{r}, t)|^{2}$ over the period $2 \pi / \omega_{j}$ of fast oscillations, treating $c_{0}$ and $c_{j}$ as slow functions of time. The result is the envelope density

$$
\bar{\rho}(\mathbf{r}, t)=\bar{\rho}_{0}(\mathbf{r}, t)+\bar{\rho}_{j}(\mathbf{r}, t),
$$

in which

$$
\bar{\rho}_{p}(\mathbf{r}) \equiv N n_{p}(t)\left|\varphi_{p}(\mathbf{r})\right|^{2}, \quad p=0, j .
$$

The density (15.52) is normalized to the number of atoms,

$$
\int \rho(\mathbf{r}, t) d \mathbf{r}=N
$$

For a cylindrical trap, one may pass to the dimensionless notation of section 9.4 and define the dimensionless densities

$$
\rho(r, \varphi, z, t) \equiv \frac{l_{r}^{3}}{N} \bar{\rho}(\mathbf{r}, t), \quad \rho_{n}(r, \varphi, z, t) \equiv \frac{l_{r}^{3}}{N} \bar{\rho}_{n}(\mathbf{r}, t)
$$

depending on the dimensionless space variables (9.24). The introduced dimensionless density is normalized as

$$
\int_{0}^{\infty} \int_{0}^{2 \pi} \int_{-\infty}^{+\infty} \rho(r, \varphi, z, t) r d r d \varphi d z=1
$$

and is given by the sum

$$
\rho(r, \varphi, z, t)=\rho_{0}(r, \varphi, z, t)+\rho_{j}(r, \varphi, z, t)
$$

in which

$$
\rho_{0}(r, \varphi, z, t) \equiv n_{0}(t)\left|\psi_{0}(r, \varphi, z)\right|^{2}, \quad \rho_{j}(r, \varphi, z, t) \equiv n_{j}(t)\left|\psi_{j}(r, \varphi, z)\right|^{2},
$$

with $\psi_{0}$ and $\psi_{j}$ being the dimensionless coherent modes. 
In the optimized approximation of Chapter 10, the ground state mode can be written as

$$
\psi_{000}(r, \varphi, z)=\left(\frac{u^{2} v}{\pi^{3}}\right)^{1 / 4} \exp \left\{-\frac{1}{2}\left(u r^{2}+v z^{2}\right)\right\}
$$

where the control functions $u=u_{000}$ and $v=v_{000}$, in the strong-coupling limit, are

$$
u_{000}=\frac{\left(2 \pi^{3}\right)^{1 / 5}}{(\nu g)^{2 / 5}}, \quad v_{000}=\frac{\left(2 \pi^{3}\right)^{1 / 5} \nu^{2}}{(\nu g)^{2 / 5}} .
$$

The ground-state energy is

$$
E_{000}=\frac{5(\nu g)^{2 / 5}}{4\left(2 \pi^{3}\right)^{1 / 5}}=0.547538(\nu g)^{2 / 5}
$$

where the indices mean $n=0, m=0, k=0$, and again the strong-coupling condition $\nu g \gg 1$ is assumed.

The radial dipole mode, with the quantum numbers $n=1, m=k=0$, is presented by

$$
\psi_{100}(r, \varphi, z)=\left(\frac{u^{2} v}{\pi^{3}}\right)^{1 / 4}\left(u r^{2}-1\right) \exp \left\{-\frac{1}{2}\left(u r^{2}+v z^{2}\right)\right\}
$$

where $u=u_{100}$ and $v=v_{100}$ are

$$
u_{100}=\frac{(6 \pi)^{3 / 5}}{(\nu g)^{2 / 5}}, \quad v_{100}=\frac{(6 \pi)^{3 / 5} \nu^{2}}{3(\nu g)^{2 / 5}} .
$$

The corresponding energy writes

$$
E_{100}=\frac{5}{8}\left(\frac{36}{\pi^{3}}\right)^{1 / 5}(\nu g)^{2 / 5}=0.643948(\nu g)^{2 / 5} .
$$

Therefore, the transition frequency is

$$
\omega_{100} \equiv E_{100}-E_{000}=0.096410(\nu g)^{2 / 5} .
$$

Here and in what follows the strong-coupling limit $\nu g \gg 1$ is again supposed.

The vortex mode, with the quantum numbers $n=0, m=1, k=0$, is of the form

$$
\psi_{010}(r, \varphi, z)=u\left(\frac{v}{\pi^{3}}\right)^{1 / 4} r e^{i \varphi} \exp \left\{-\frac{1}{2}\left(u r^{2}+v z^{2}\right)\right\},
$$

where $u=u_{010}, v=v_{010}$ are

$$
u_{010}=\frac{2\left(2 \pi^{3}\right)^{1 / 5}}{(\nu g)^{2 / 5}}, \quad v_{010}=\frac{\left(2 \pi^{3}\right)^{1 / 5} \nu^{2}}{(\nu g)^{2 / 5}} .
$$

The transition frequency from the ground to the vortex state is

$$
\omega_{010} \equiv E_{010}-E_{000}=\frac{3.424}{(\nu g)^{2 / 5}} .
$$

The axial dipole mode, with the quantum numbers $n=0, m=0, k=1$ reads

$$
\psi_{001}(r, \varphi, z)=\left(\frac{4 u^{2} v^{3}}{\pi^{3}}\right)^{1 / 4} z \exp \left\{-\frac{1}{2}\left(u r^{2}+v z^{2}\right)\right\},
$$

where $u=u_{001}, v=v_{001}$ are

$$
u_{001}=\frac{2(\pi / 3)^{3 / 5}}{(\nu g)^{2 / 5}}, \quad v_{001}=\frac{6(\pi / 3)^{3 / 5} \nu^{2}}{(\nu g)^{2 / 5}}
$$


The related energy is

$$
E_{001}=\frac{5}{8}\left(\frac{3}{\pi}\right)^{3 / 5}(\nu g)^{2 / 5}=0.607943(\nu g)^{2 / 5} .
$$

Hence, for the transition frequency, one has

$$
\omega_{001} \equiv E_{001}-E_{000}=0.060405(\nu g)^{2 / 5} .
$$

The spatio-temporal behavior of the densities (15.55) for low-lying modes is illustrated in Figs. 38 to 40. The corresponding wave functions are taken from Eqs. (15.56), (15.58), (15.61), and (15.63). The fractional populations (15.15) are calculated by solving Eqs. (15.16).

\subsection{Resonance Formation of Vortices}

To form a vortex, the resonance field (15.3) must depend on the radial angle, so that the corresponding transition amplitude (15.12) be nonzero. For the latter, employing the dimensionless cylindrical variables, one has

$$
\beta_{n m k}=\frac{1}{\hbar} \int_{0}^{\infty} r d r \int_{0}^{2 \pi} d \varphi \int_{-\infty}^{+\infty} d z \psi_{0}(r, \varphi, z) V(r, \varphi, z) \psi_{n m k}(r, \varphi, z) .
$$

In the case of a pure vortex, with $n=k=0$ and $m \neq 0$, using for the ground state the function $(15.56)$ and for the vortex mode

$$
\psi_{0 m 0}(r, \varphi, z)=\left(\frac{u_{m}^{|m|+1}}{\pi|m| !}\right)^{1 / 2}\left(\frac{v_{m}}{\pi}\right)^{1 / 4} r^{|m|} \exp \left\{-\frac{1}{2}\left(u_{m} r^{2}+v_{m} z^{2}\right)+i m \varphi\right\},
$$

with the control functions $u_{m} \equiv u_{0 m 0}$ and $v_{m} \equiv v_{0 m 0}$, one finds

$$
\begin{gathered}
\beta_{0 m 0}=\frac{1}{\pi \hbar}\left(\frac{2 u_{0} u_{m}^{|m|+1}}{|m| !}\right)^{1 / 2} \frac{\left(v_{0} v_{m}\right)^{1 / 4}}{\left(v_{0}+v_{m}\right)^{1 / 2}} \times \\
\times \int_{0}^{\infty} r d r \int_{0}^{2 \pi} d \varphi V(r, \varphi) r^{|m|} \exp \left\{-\frac{1}{2}\left(u_{0}+u_{m}\right) r^{2}+i m \varphi\right\} ;
\end{gathered}
$$

the resonant field being assumed to depend only on $r$ and $\varphi$. Taking this field in the form

$$
V(r, \varphi)=\frac{\kappa}{p !} \hbar \omega_{r} r^{p} \exp \left(-i m^{\prime} \varphi\right)
$$

which corresponds to the rotating potential (15.3) with $V_{1}(\mathbf{r}) \sim \cos m^{\prime} \varphi$ and $V_{2}(\mathbf{r}) \sim \sin m^{\prime} \varphi$, one obtains

$$
\beta_{0 m 0}=\delta_{m m^{\prime}} \frac{\kappa \omega_{r}}{p !} \Gamma\left(1+\frac{p+|m|}{2}\right)\left(\frac{u_{0} u_{m}^{|m|+1}}{|m| ! \bar{u}^{p+|m|+2}}\right)^{1 / 2}\left(\frac{v_{0} v_{m}}{\bar{v}^{2}}\right)^{1 / 4},
$$

where

$$
\bar{u} \equiv \frac{1}{2}\left(u_{0}+u_{m}\right), \quad \bar{v} \equiv \frac{1}{2}\left(v_{0}+v_{m}\right) .
$$

For some particular cases, when $m^{\prime}=1$ and $p=0,1,2$, we get

$$
\begin{aligned}
& \beta_{0 m 0}=\delta_{m 1} 0.964 \kappa \omega_{r} \quad(p=0), \\
& \beta_{0 m 0}=\delta_{m 1} 0.588 \kappa \omega_{r}(\nu g)^{1 / 5} \quad(p=1), \\
& \beta_{0 m 0}=\delta_{m 1} 0.598 \kappa \omega_{r}(\nu g)^{2 / 5} \quad(p=2) .
\end{aligned}
$$

Expression (15.70) shows that the modulating field (15.69) will excite vortices with the winding numbers $m$. 


\subsection{Problems in Resonance Excitation}

In deriving the evolution equations (15.16) for an effective two-level system, an essential assumption was made that the coefficients $c_{n}$ are slow functions of time, so that the inequality (15.9) holds true. The transition amplitude (15.20) of the resonant field can always be taken so that $\beta<\alpha$. Then Eqs. (15.15) show that the time variation $d c_{n} / d t$ is of order $\alpha$. Hence, it should be that $|\alpha| \ll E_{j}$. From the definition (15.13) it follows that

$$
\alpha=2 g \omega_{r} \int_{0}^{\infty} r d r \int_{0}^{2 \pi} d \varphi \int_{-\infty}^{+\infty} d z\left|\psi_{0}(r, \varphi, z)\right|^{2}\left(2\left|\psi_{j}(r, \varphi, z)\right|^{2}-\left|\psi_{0}(r, \varphi, z)\right|^{2}\right)
$$

where the index $j$ implies the triplet of quantum numbers $n, m, k$. Calculations show that for $g \nu \gg 1$ the value of $\alpha$ can become of order $E_{j}$. This means that the two-level picture can be a rather rough approximation for $g \nu \gg 1$, and one would expect the ground state to be coupled to more than one excited mode. Such a situation is analogous to the effect of power broadening in optics [296]. In order for the two-level picture to be a good approximation, one should choose $g \nu$ not too large. The atom-atom coupling parameter $g$, defined in Eq. (9.27), is proportional to the number of atoms $N$. If the number of atoms in the coherent state is large, $N \gg 1$, then it may be that $g \gg 1$. Hence, to reduce the value of the product $g \nu$, one has to take small $\nu$, making $g \nu \sim 1$. Small $\nu$ implies that the trap should have the shape of a long cylinder.

In order to check directly that the two-level picture is a reasonable first approximation, it is necessary to solve numerically the time-dependent equation (15.1). The latter, in the dimensionless units of section 9.4, acquires the form

$$
i \frac{\partial \psi}{\partial t}=\left(\hat{H}+\hat{V}_{\text {res }}\right) \psi
$$

where $\psi=\psi(\mathbf{r}, t)$; time is measured in units of $\omega_{r}^{-1} ; \mathbf{r}=\{r, \varphi, z\}$, with the dimensionless cylindrical variables $r \in[0, \infty), \varphi \in[0,2 \pi]$, and $z \in(-\infty,+\infty)$. The Hamiltonian reads

$$
\hat{H}=-\frac{1}{2} \nabla^{2}+\frac{1}{2}\left(r^{2}+\nu^{2} z^{2}\right)+g|\psi|^{2},
$$

where

$$
\nabla^{2}=\frac{\partial^{2}}{\partial r^{2}}+\frac{1}{r} \frac{\partial}{\partial r}+\frac{1}{r^{2}} \frac{\partial^{2}}{\partial \varphi^{2}}+\frac{\partial^{2}}{\partial z^{2}} .
$$

The resonant field $\hat{V}_{\text {res }}$, measured in units of $\hbar \omega_{r}$, can be taken in one of the following forms, depending on the type of a topological mode one would wish to excite: Thus, the modulating field

$$
\hat{V}_{\text {res }}=\kappa r \cos \omega t
$$

is needed for exciting the radial dipole mode. The field

$$
\hat{V}_{\text {res }}=\frac{\kappa r^{p}}{p !}[\cos (m \varphi) \cos \omega t+\sin (m \varphi) \sin \omega t],
$$

with $p=0,1,2, \ldots$, is sufficient for exciting vortices with the winding number $m$. And the resonance field

$$
\hat{V}_{\text {res }}=\kappa z \cos \omega t
$$

will excite the axial dipole mode. Choosing the appropriate modulating field, one can create the related topological mode.

As initial condition to Eq. (15.72), one has to take the ground-state mode that can be approximately presented as

$$
\psi(\mathbf{r}, t)=\left(\frac{u^{2} v}{\pi^{3}}\right)^{1 / 4} \exp \left\{-\frac{1}{2}\left(u r^{2}+v z^{2}\right)\right\} .
$$

Here, the control functions $u$ and $v$ are defined by Eqs. (10.52, which, for the ground-state case, reduce to

$$
\left(1-\frac{1}{u^{2}}\right)+\frac{s}{\nu} \sqrt{v}=0, \quad\left(1-\frac{\nu^{2}}{v^{2}}\right)+\frac{s}{\nu \sqrt{v}}=0
$$


with the variable $s=2 \nu g /(2 \pi)^{3 / 2}$.

The resonance effect in the two-level picture can be noticed as follows. One may observe the spatio-temporal behavior of the dimensionless density

$$
n(r, \varphi, z, t) \equiv|\psi(r, \varphi, z, t)|^{2},
$$

studying the radial and axial cross-sections, $n(r, 0,0, t)$ and $n(0,0, z, t)$. The appearance of excited topological modes, with the spatial shape qualitatively different from the ground-state mode (15.77), should be noticed in the corresponding cross-sections of the density (15.78).

The formation of a vortex can also be noticed by studying the angular orbital momentum

$$
L_{z}=-i \int \psi^{*} \frac{\partial}{\partial \varphi} \psi d \mathbf{r}
$$

If there are no vortices, $L_{z}=0$, while when there appears a vortex with the winding number $m$, then $L_{z}=m$. Because of the oscillatory character of the problem, the orbital momentum will also oscillate since $L_{z}=L_{z}(t)$ is a function of time. One may consider the temporal behavior of $L_{z}(t)$. If in some moments of time the latter reaches an integer value $m$, this would mean that there occurs the formation of a vortex with the winding number $m$.

The problem of numerical solution can be simplified in three particular cases. The first case is when one is interested in exciting the radial dipole mode in a long cylindrical trap, for which $\nu \ll 1$. Then one can limit oneself to the consideration of the wave function behavior at the center $z=0$, assuming that at this center, the wave function practically does not depend on $z$ and $\varphi$. This permits one to neglect the derivatives over $z$ and $\varphi$ in Eq. (15.72), which yields

$$
i \frac{\partial \psi}{\partial t}=\left(\hat{H}_{r}+\hat{V}_{\text {res }}\right) \psi
$$

where $\psi=\psi(r, t) \equiv \psi(r, 0,0, t)$ and the radial nonlinear Hamiltonian is

$$
\hat{H}_{r}=-\frac{1}{2}\left(\frac{\partial^{2}}{\partial r^{2}}+\frac{1}{r} \frac{\partial}{\partial r}\right)+\frac{1}{2} r^{2}+g|\psi|^{2} .
$$

To excite the radial dipole mode, one has to use the modulating field (15.74).

Another case of simplifying the computational problem is when one investigates the excitation of a vortex mode in a long cylindrical trap, so that again $\nu \ll 1$. Then considering, as previously, the problem at the center $z=0$, one may assume that the wave function slowly changes. The latter allows one to omit the derivative over $z$, which simplifies Eq. (15.72) to

$$
i \frac{\partial \psi}{\partial t}=\left(\hat{H}_{\perp}+\hat{V}_{r e s}\right) \psi,
$$

with the function $\psi=\psi(r, \varphi, t) \equiv \psi(r, \varphi, 0, t)$ and the transverse Hamiltonian

$$
\hat{H}_{\perp}=-\frac{1}{2} \nabla_{\perp}^{2}+\frac{1}{2} r^{2}+g|\psi|^{2},
$$

in which

$$
\nabla_{\perp}^{2}=\frac{\partial^{2}}{\partial r^{2}}+\frac{1}{r} \frac{\partial}{\partial r}+\frac{1}{r^{2}} \frac{\partial^{2}}{\partial \varphi^{2}} .
$$

For the excitation of the vortex mode, one should take the resonant field (15.75).

Finally, one may consider the excitation of the axial dipole mode in a disk-shaped trap, with $\nu \gg 1$. It is then admissible to analyze the situation at the axis $r=0$, assuming the slow dependence of the wave function on $r$ and $\varphi$. This changes Eq. (15.72) to

$$
i \frac{\partial \psi}{\partial t}=\left(\hat{H}_{z}+\hat{V}_{\text {res }}\right) \psi
$$


with $\psi=\psi(z, t) \equiv \psi(0,0, z, t)$ and the axial Hamiltonian

$$
\hat{H}_{z}=-\frac{1}{2} \frac{\partial^{2}}{\partial z^{2}}+\frac{1}{2} \nu^{2} z^{2}+g|\psi|^{2} .
$$

The axial dipole mode is to be excited by the modulating resonant field (15.76).

In all these simplified cases, as well as for the general equation (15.72), one has to set boundary conditions in addition to the considered initial conditions. These boundary conditions are rather obvious, and for the general wave function $\psi(r, \varphi, z, t)$ they write

$$
\begin{gathered}
\lim _{r \rightarrow \infty} \psi(r, \varphi, z, t)=0, \quad \lim _{z \rightarrow \pm \infty} \psi(r, \varphi, z, t)=0, \\
\psi(r, \varphi+2 \pi, z, t)=\psi(r, \varphi, z, t) .
\end{gathered}
$$

Numerical investigation of the equations discussed in this section is yet in process. However, the validity of the two-level picture has been proved by direct numerical calculations for several similar problems 1140,379 , 380, 398, 404, where the nonlinear Rabi-type oscillations between the ground-state and an excited mode have been clearly observed.

Another interesting problem would be to study the possibility and peculiarity of the resonance formation of coherent topological modes in Bose-Einstein condensates with attractive interactions. Such condensates exhibit oscillatory collective collapse [111. Being subject to a resonant modulating field, the condensate should also show the nonlinear Rabi oscillations. These two kinds of oscillations should interfere resulting in a rather intricate behavior. It could, probably, be possible to regulate the oscillating collapse by means of a resonant field. 


\section{Chapter 16}

\section{Coherence and Atom Lasers}

The possibility of realizing Bose-Einstein condensation in trapped dilute gases demonstrates, that a macroscopic number of bosons can be produced in a single quantum state of trapped atoms. The occupation of a single quantum state by a large number of bosons is the matter-wave analog of the storage of photons in a single mode of a laser cavity. A device that could emit coherent beams of Bose atoms, similarly to the emission of photon rays by light lasers, can be called atom laser 196-203. Briefly speaking, an atom laser can be defined as a device emitting highly-directional beams of coherent atoms. Therefore, there are two principal questions related to the realization of atom lasers: whether the stored bosons are prepared in a coherent state and how to form a well-collimated beam of atoms in any desired direction.

\subsection{Interference and Josephson Effect}

As shown in Chapter 8, Bose-Einstein condensation in dilute gases of trapped atoms can be understood as the macroscopic occupation of the ground-state coherent mode. An important consequence of coherence is the occurrence of interference phenomena. These have been observed in a nice experiment 195, which confirms that Bose-Einstein condensed trapped atoms are in a coherent state. In this experiment, a laser beam was used to cut a cigar-shaped atomic cloud into two spatially separated parts. After switching off the confining potential and the laser, the two independent atomic clouds fall down because of gravity, expand because of atomic repulsion, and eventually overlap. Clean interference patterns have been observed in the overlapping region.

The appearance of interference patterns can be easily explained as follows. Imagine that a cloud of atoms is separated into two parts whose locations are centered at $\mathbf{l}_{1}$ and $\mathbf{l}_{2}$. Being released from the trap, these parts move with the corresponding velocities $\mathbf{V}_{1}$ and $\mathbf{V}_{2}$. Then the field operator can be presented as

$$
\psi(\mathbf{r}, t)=\psi_{1}(\mathbf{r}, t) e^{i \mathbf{k}_{1} \cdot \mathbf{r}}+\psi_{2}(\mathbf{r}, t) e^{i \mathbf{k}_{2} \cdot \mathbf{r}},
$$

where $\psi_{1}$ and $\psi_{2}$ are the field operators of the separated immovable parts, and $\hbar \mathbf{k}_{j} \equiv m_{0} \mathbf{V}_{j}$.

The interference pattern can be described by the function

$$
I(\mathbf{r}, t) \equiv \rho(\mathbf{r}, t)-\rho_{1}(\mathbf{r}, t)-\rho_{2}(\mathbf{r}, t),
$$

in which

$$
\rho(\mathbf{r}, t) \equiv<\psi^{\dagger}(\mathbf{r}, t) \psi(\mathbf{r}, t)>, \quad \rho_{j}(\mathbf{r}, t) \equiv<\psi_{j}^{\dagger}(\mathbf{r}, t) \psi_{j}(\mathbf{r}, t)>.
$$

From Eqs. (16.1) and (16.2) it follows that

$$
I(\mathbf{r}, t)=2 \operatorname{Re} \rho_{12}(\mathbf{r}, t) e^{-\mathbf{k}_{12} \cdot \mathbf{r}},
$$

where $\mathbf{k}_{12} \equiv \mathbf{k}_{1}-\mathbf{k}_{2}$ and

$$
\rho_{12}(\mathbf{r}, t) \equiv<\psi_{1}^{\dagger}(\mathbf{r}, t) \psi_{2}(\mathbf{r}, t)>
$$


The initial separation of the cloud parts is assumed to be much larger than the mean interatomic distance,

$$
l_{12} \gg a, \quad l_{12} \equiv\left|\mathbf{l}_{1}-\mathbf{l}_{2}\right| .
$$

If atoms are not in a coherent state, so that the coherence length is small, $r_{c o h} \leq a$, then the correlation function (16.5) is practically zero, together with the interference function (16.4). Hence, no interference can be observed. When there exists local coherence, so that $r_{c o h} \gg a$, then the correlation function (16.5) does not decay so fast, and the observation of interference becomes possible. If almost the whole system was initially in a coherent state, so that $r_{c o h} \sim L>l_{12}$, then the correlation function (16.5) takes the form

$$
\rho_{12}(\mathbf{r}, t)=\rho_{12}(\mathbf{r}) e^{i \omega_{12} t}
$$

in which $\hbar \omega_{12} \equiv E_{1}-E_{2}$, with $E_{j}$ being the energy of the coherent mode related to a $j$-part, and

$$
\rho_{12}(\mathbf{r}) \equiv N \varphi_{1}^{*}(\mathbf{r}) \varphi_{2}(\mathbf{r}),
$$

where $\varphi_{j}$ is a coherent mode located at $\mathbf{l}_{j}$. If atoms are in the ground state, then the modes $\varphi_{j}$ are real. Consequently, $\rho_{12}(\mathbf{r})$ is also real. Therefore, the interference function (16.4) becomes

$$
I(\mathbf{r}, t)=2 \rho_{12}(\mathbf{r}) \cos \left(\mathbf{k}_{12} \cdot \mathbf{r}-\omega_{12} t\right),
$$

and one can observe clean interference patterns. In general, these patterns will display collapses and revivals with the period

$$
\Delta t=\frac{2 \pi}{\omega_{12}} .
$$

But if the energies of both separated parts are the same, $E_{1}=E_{2}$, then $\omega_{12}=0$, and the interference pattern is stationary, with the interference function

$$
I(\mathbf{r}, t)=2 \rho_{12}(\mathbf{r}) \cos \left(\mathbf{k}_{12} \cdot \mathbf{r}\right) .
$$

The spatial interference can be characterized by the interference fringe spacing. Considering, say the $x$ direction, one may define the fringe period $\Delta x=2 \pi / k_{12}$. With the evident renotation

$$
\hbar k_{12}=m_{0} V_{12}, \quad V_{12}=\frac{l_{12}}{t},
$$

one obtains the fringe period

$$
\Delta x=2 \pi \frac{\hbar t}{m_{0} l_{12}},
$$

in agreement with the experiment [195] and with the discussion in Ref. 405].

It is worth emphasizing that coherence is the necessary and sufficient condition for interference. And this requires no breaking of gauge symmetry, so that one can set $\langle\psi\rangle=0$, as is elucidated in Chapter 8 . The assumption of broken gauge symmetry is only a sufficient condition for interpreting interference, but it is not a necessary condition. Therefore it is not correct to state, as many do, that the observation of interference proves the existence of broken gauge symmetry. Such a statement is wrong, since one presupposes what is alleged to be proved.

Another manifestation of coherence in trapped condensates could be the possible occurrence of Josephsontype effects, in analogy with the known properties of Josephson junctions in superconductors and superfluids. To work out the physical idea, we consider again two separated condensates confined in a double-well trap which erects a barrier between them. Then the field operator can be written as

$$
\psi(\mathbf{r}, t)=\psi_{1}(\mathbf{r}, t)+\psi_{2}(\mathbf{r}, t),
$$

similarly to Eq. (16.1), but with $\mathbf{k}_{j}=0$, if the condensate on average does not moves. The physical meaning of the Josephson effect is the manifestation of interference in the atomic current, equivalently to the manifestation 
of interference in the atomic density, which is described by the interference density function (16.2). Thus, the interference current is defined as

$$
\mathbf{J}(\mathbf{r}, t)=<\mathbf{j}(\mathbf{r}, t)>-<\mathbf{j}_{1}(\mathbf{r}, t)>-<\mathbf{j}_{2}(\mathbf{r}, t)>,
$$

where

$$
\mathbf{j}(\mathbf{r}, t) \equiv-\frac{i \hbar}{2 m_{0}}\left[\psi^{\dagger} \boldsymbol{\nabla} \psi-\left(\boldsymbol{\nabla} \psi^{\dagger}\right) \psi\right], \quad \mathbf{j}_{i}(\mathbf{r}, t) \equiv-\frac{i \hbar}{2 m_{0}}\left[\psi_{i}^{\dagger} \nabla \psi_{i}-\left(\boldsymbol{\nabla} \psi_{i}^{\dagger}\right) \psi_{i}\right] .
$$

With the split operator (16.13), the current (16.14) is

$$
\mathbf{J}(\mathbf{r}, t)=2 \operatorname{Re}<\mathbf{j}_{12}(\mathbf{r}, t)>,
$$

where

$$
\mathbf{j}_{12}(\mathbf{r}, t) \equiv-\frac{i \hbar}{2 m_{0}}\left[\psi_{1}^{\dagger} \boldsymbol{\nabla} \psi_{2}-\left(\boldsymbol{\nabla} \psi_{1}^{\dagger}\right) \psi_{2}\right] .
$$

The following argumentation is the same as when considering the density interference. One assumes that the potential wells, separating the condensate in two parts, are located sufficiently far from each other, in the sense of the inequality (16.6). Then, if the system is not coherent, the correlation function $\left.<\mathbf{j}_{12}\right\rangle$ is negligible, and there is no interference current. However, if the atomic system is in a coherent state, then the coherent average gives

$$
<\mathbf{j}_{12}(\mathbf{r}, t)>_{N}=-i \mathbf{J}_{12}(\mathbf{r}) e^{i \omega_{12} t},
$$

with $\hbar \omega_{12} \equiv E_{1}-E_{2}$ and

$$
\mathbf{J}_{12}(\mathbf{r}) \equiv \frac{\hbar N}{2 m_{0}}\left[\varphi_{1}^{*}(\mathbf{r}) \nabla \varphi_{2}(\mathbf{r})-\varphi_{2}(\mathbf{r}) \nabla \varphi_{1}^{*}(\mathbf{r})\right] .
$$

For the ground state, $\varphi_{j}$ are real, so is $\mathbf{J}_{12}(\mathbf{r})$. Therefore, the interference current (16.16) becomes

$$
\mathbf{J}(\mathbf{r}, t)=2 \mathbf{J}_{12}(\mathbf{r}) \sin \omega_{12} t
$$

which is the typical Josephson form. This current depends on time only if $\omega_{12} \neq 0$, so that the energies $E_{1}$ and $E_{2}$ should be different.

Note that for a coherent state, the average $\left\langle\mathbf{j}_{i}\right\rangle_{N}$ does not depend on time, and for the ground state, when $\varphi_{i}$ are real, $\left\langle\mathbf{j}_{i}>_{N}=0\right.$. But the interference current has been defined as in Eq. (16.14) for generality and for closer analogy with the interference density (16.2).

\subsection{Conditions on Atom Lasers}

Defining an atom laser as a device emitting highly-directional beams of coherent atoms, one always assumes 202] that the very first condition on a laser is that its output is a well-collimated beam that can be pointed in an arbitrary direction:

(1) Highly-directional beam.

This condition allows one to distinguish a longitudinal direction of propagation and two transverse directions of diffraction. Good collimation implies the smallness of the aspect ratio

$$
\left|\frac{r(t)}{z(t)}\right| \ll 1
$$

in which $r(t)$ is the average transverse radius of a beam and $z(t)$, its length. The directionality also supposes that it can be chosen arbitrarily in space.

Characterizing the coherence of a laser, it is useful to slightly generalize the notions introduced in section 8.5. Coherence is intimately related to strong interatomic correlations. The information about the latter is hidden in the correlation function

$$
C\left(\mathbf{r}, \mathbf{r}^{\prime}, t\right) \equiv \frac{<\psi^{\dagger}(\mathbf{r}, t) \psi\left(\mathbf{r}^{\prime}, 0\right)>}{\sqrt{\rho(\mathbf{r}, 0) \rho\left(\mathbf{r}^{\prime}, 0\right)}},
$$


where the density $\rho(\mathbf{r}, t)$ is the same as in Eq. (16.3).

The coherence length can be defined as

$$
r_{c o h}(t) \equiv \frac{\int r|C(\mathbf{r}, 0, t)| d \mathbf{r}}{\int|C(\mathbf{r}, 0, t)| d \mathbf{r}}
$$

and the coherence time as

$$
\tau_{c o h}(\mathbf{r}) \equiv \int_{0}^{\infty}|C(\mathbf{r}, 0, t)| d t
$$

As is seen, the coherence length is, generally, a function of time, while the coherence time depends on the spatial variable. One may distinguish spatial and temporal coherence.

(2) Spatial coherence.

This requires that for some period of time,

$$
r_{c o h}(t) \gg a
$$

where $a$ is the mean interatomic distance. It is not compulsory that the inequality (16.25) be valid for all times, but it is sufficient that it holds true during the time of beam emission. Thus, for a pulsing laser, this should be the time of emitting one beam.

(3) Temporal coherence.

This is the condition on the coherence time,

$$
\tau_{c o h}(\mathbf{r}) \gg \gamma^{-1}
$$

where $\gamma$ is a spectrum linewidth. Temporal coherence is related to the condition of monochromaticity,

$$
\gamma \ll \omega,
$$

with $\hbar \omega$ being a characteristic atomic energy.

A simple model for an atom laser can be formulated as follows 203. Assume that not all atoms of the system are in a coherence state, but only $N_{c o h}$ of them, so that the coherent field $\eta$ is normalized as

$$
N_{c o h}=(\eta, \eta) .
$$

A part of atoms, $N_{i n c}$, is not coherent, for example because of fluctuations [406] or because of depolarizing collisions 363. In order to take into account that the number of atoms in a trap is not conserved, one should add to the equation for the coherent field the terms describing atom loss and gain. This can be done by adding to the nonlinear Hamiltonian (8.17) the corresponding terms

$$
H_{\text {gain }} \equiv \frac{i}{2} \hbar \gamma_{+} N_{\text {inc }}, \quad H_{\text {loss }} \equiv-\frac{i}{2} \hbar \gamma_{-} .
$$

Then the evolution equation 8.16 for the coherent field transforms to

$$
i \hbar \frac{\partial \eta}{\partial t}=\left(-\frac{\hbar^{2}}{2 m_{0}} \nabla^{2}+U\right) \eta+A|\eta|^{2} \eta+\frac{i}{2} \hbar\left(\gamma_{+} N_{i n c}-\gamma_{-}\right) \eta .
$$

From here, with the normalization (16.28), it is straightforward to get the rate equation

$$
\frac{d}{d t} N_{c o h}=\left(\gamma_{+} N_{i n c}-\gamma_{-}\right) N_{c o h}
$$

The latter is to be complemented by the rate equation for incoherent atoms, which can be taken in the form

$$
\frac{d}{d t} N_{i n c}=P-\left(\gamma_{+} N_{i n c}+\Gamma\right) N_{i n c},
$$


where $P$ is a pumping or generation rate and $\Gamma$ is a loss rate.

Analyzing the stationary solutions to the rate equations (16.31) and (16.32), one finds that two regimes exist, depending on the value of the generation rate $P$ as compared to the critical threshold quantity

$$
P_{c} \equiv \frac{\gamma_{-}}{\gamma_{+}} \Gamma \text {. }
$$

For low generation rates, the stable stationary solutions are

$$
N_{c o h}^{*}=0, \quad N_{i n c}^{*}=\frac{P}{\Gamma} \quad\left(P<P_{c}\right),
$$

hence, there is no stationary generation of coherent atoms. But as soon as the generation rate $P$ exceeds the threshold (16.33), the stable stationary solutions become

$$
N_{c o h}^{*}=\frac{P-P_{c}}{\gamma_{-}}, \quad N_{i n c}^{*}=\frac{\gamma_{-}}{\gamma_{+}} \quad\left(P>P_{c}\right) .
$$

Then the steady-state number of atoms in the condensate grows linearly with the pump rate $P$. This situation reminds the lasing threshold for generation in photon lasers.

The model outlined above has not addressed details of the output coupling, simply assuming the existence of a loss mechanism from the lasing mode. But output coupling obviously constitutes a vital element of an atom laser. The general idea of realizing output coupling is to transfer atoms, via a radiofrequency or microwave field, from a trapped state to an untrapped state. Being transferred to a state that is not confined by magnetic fields, the atoms would fly out in all directions, if gravity would not force them to fall down.

Mewes et al. [14] have experimentally demonstrated precisely such an output coupler for Bose-condensed sodium atoms. Using short resonant pulses of radiofrequency radiation, an arbitrary percentage of the atomic population could be transferred in a controllable manner to the output state. Atoms in the output state simply fall down from the trap under the action of gravity. Bloch et al. [16] have demonstrated a continuous output coupler for magnetically trapped rubidium atoms. Over a period of up to $100 \mathrm{~ms}$, atoms could be continuously extracted from condensate by a weak radiofrequency field inducing spin flips between trapped and untrapped states. In the untrapped state, the atoms leak out of the trap, experiencing the action of gravity. Hagley et al. [17] extracted sodium atoms from a trapped condensate using stimulated Raman transitions between magnetic sublevels. In the latter experiment [17, contrary to the previous ones [14, 16], optical Raman pulses drove transitions between trapped and untrapped magnetic sublevels, giving the output-coupled fraction of atoms a well-defined momentum kick from the photon recoil. Because of this, atoms exited the trap in a well-defined beam whose direction could be varied via the details of the Raman lasers. This technique produced a device that could really be called an atom laser, since the orientation of the laser beam did not rely on gravity but could be selected 407.

\subsection{Nonadiabatic Dynamics of Atoms}

The motion of trapped atoms is usually described as being governed by an effective confining potential. Such a picture is equivalent to the adiabatic approximation that is applicable for describing the stationary motion of atoms. But when atoms escape from a trap, their motion is, certainly, not stationary and hence, in general, it is not necessarily adiabatic. The study of nonadiabatic dynamics of atoms is not only useful because this gives a more general picture of atomic motion, but also because in this more general picture some novel dynamical regimes could be found, suggesting new mechanisms for creating highly-directional beams from atom lasers.

To derive general equations of atomic motion in a trap, one should start not with an ad hoc introduced effective confining potential but with the consideration of the real forces in the trap. For this purpose, one can invoke a quantum-mechanical description based on the Hamiltonian

$$
\hat{H}_{N}=\sum_{i=1}^{N}\left(\frac{\mathbf{p}_{i}^{2}}{2 m_{0}}-\mu_{0} \mathbf{S}_{i} \cdot \mathbf{B}_{i}-m_{0} \mathbf{g} \cdot \mathbf{r}_{i}\right)+\frac{1}{2} \sum_{i \neq j} \Phi_{i j}
$$


for a system of $N$ atoms, in which $\mu_{0}$ is an atomic magnetic moment, $\mathbf{p}_{i}=-i \hbar \boldsymbol{\nabla}$ is a momentum operator, $\mathbf{S}_{i}$ is a spin operator, $\mathbf{B}_{i}$ is the magnetic field formed by the trap, $\mathbf{g}$ is the gravitational acceleration, and $\Phi_{i j}$ is an interaction potential. The evolution of this system is given by the wave function $\Psi_{N}=\Psi_{N}\left(\mathbf{r}_{1}, \mathbf{r}_{2}, \ldots, \mathbf{r}_{N}, t\right)$ satisfying the Schrödinger equation

$$
i \hbar \frac{\partial}{\partial t} \Psi_{N}=\hat{H}_{N} \Psi_{N}
$$

Note that $\Psi_{N}=\left[\Psi_{N}^{\sigma}\right]$ is a column in the space of spin variables. For an operator $\hat{A}$ from the algebra of observables, the quantum-mechanical average is given by the scalar product

$$
<\hat{A}>\equiv\left(\Psi_{N}, \hat{A} \Psi_{N}\right)
$$

The temporal behavior of this average follows from the Schrödinger equation giving

$$
\frac{d}{d t}<\hat{A}>=<\frac{\partial \hat{A}}{\partial t}>+\frac{i}{\hbar}<\left[\hat{H}_{N}, \hat{A}\right]>.
$$

In particular cases, this yields the so-called Ehrenfest equations, that is the equations for the mean space variable,

$$
\frac{d}{d t}<\mathbf{r}_{i}>=\frac{1}{m_{0}}<\mathbf{p}_{i}>
$$

and for the mean momentum of one atom,

$$
\frac{d}{d t}<p_{i}^{\alpha}>=\mu_{0}<\mathbf{S}_{i} \cdot \frac{\partial \mathbf{B}_{i}}{\partial r_{i}^{\alpha}}>+\mu_{0} g^{\alpha}+f^{\alpha},
$$

where $\alpha=x, y, z$ and

$$
\mathbf{f} \equiv-\sum_{j(\neq i)}^{N}<\nabla_{i} \Phi_{i j}>
$$

For the mean spin, one gets

$$
\frac{d}{d t}<\mathbf{S}_{i}>=\frac{\mu_{0}}{\hbar}<\mathbf{S}_{i} \times \mathbf{B}_{i}>.
$$

It is convenient to introduce the notation

$$
\mathbf{r} \equiv<\mathbf{r}_{i}>, \quad \mathbf{S} \equiv<\mathbf{S}_{i}>, \quad \mathbf{B} \equiv<\mathbf{B}_{i}>.
$$

To render the system of equations closed, one employs the semiclassical approximation

$$
<S_{i}^{\alpha} B_{i}^{\beta}>=S^{\alpha} B^{\beta}, \quad<\frac{\partial \mathbf{B}_{i}}{\partial r_{i}^{\alpha}}>=\frac{\partial \mathbf{B}}{\partial r^{\alpha}} .
$$

Then Eqs. (16.38) and (16.39) yield

$$
\frac{d^{2} r^{\alpha}}{d t^{2}}=\frac{\mu_{0}}{m_{0}} \mathbf{S} \cdot \frac{\partial \mathbf{B}}{\partial r^{\alpha}}+g^{\alpha}+\frac{f^{\alpha}}{m_{0}}
$$

and Eq. (16.41) results in

$$
\frac{d \mathbf{S}}{d t}=\frac{\mu_{0}}{\hbar} \mathbf{S} \times \mathbf{B} .
$$

The system of equations (16.44) and (16.45) is basic for considering the dynamics of atoms in nonuniform magnetic fields 408].

The total magnetic field of the trap can be taken as the sum

$$
\mathbf{B}=\mathbf{B}_{1}+\mathbf{B}_{2},
$$


in which the first term is the quadrupole field

$$
\mathbf{B}_{1}=B_{1}^{\prime}\left(x \mathbf{e}_{x}+y \mathbf{e}_{y}+\lambda z \mathbf{e}_{z}\right),
$$

typical of magnetic traps, where $\lambda$ is an anisotropy parameter. If the quadrupole field is formed by one pair of magnetic coils, then one has $\boldsymbol{\nabla} \cdot \mathbf{B}_{1}=0$, which gives $\lambda=-2$. However, in general, the anisotropy parameter $\lambda$ can be varied. The second term

$$
\mathbf{B}_{2}=B_{2}\left(h_{x} \mathbf{e}_{x}+h_{y} \mathbf{e}_{y}\right)
$$

where $h_{\alpha}=h_{\alpha}(t)$ and

$$
h_{x}^{2}(t)+h_{y}^{2}(t)=1
$$

is a transverse field often employed in magnetic traps to stabilize the motion of atoms.

In what follows, it is convenient to switch to the dimensionless space variable $\mathbf{r}=\{x, y, z\}$ measured in units of the characteristic length

$$
R_{0} \equiv \frac{B_{2}}{B_{1}^{\prime}}
$$

To return to the dimensional Cartesian vector, one has to make the substitution

$$
\mathbf{r} \rightarrow \frac{\mathbf{r}}{R_{0}} .
$$

Let us define the characteristic frequencies $\omega_{1}$ and $\omega_{2}$ by the equalities

$$
\omega_{1}^{2} \equiv \frac{\mu_{0} B_{1}^{\prime}}{m_{0} R_{0}}, \quad \omega_{2} \equiv \frac{\mu_{0} B_{2}}{\hbar}
$$

and introduce an effective frequency

$$
\omega \equiv \max _{t}\left|\frac{d}{d t} \mathbf{h}(t)\right|
$$

where $\mathbf{h}=\left\{h_{x}, h_{y}, 0\right\}$. Also, the notation

$$
\gamma \vec{\xi} \equiv \frac{\mathbf{f}}{m_{0} R_{0}}, \quad \mathbf{G} \equiv \frac{\mathbf{g}}{R_{0} \omega_{1}^{2}}
$$

will be used, with $\gamma$ being a collision rate.

The force (16.40) caused by pair interactions can be modelled by a random force due to pair collisions. Then $\vec{\xi}$ in Eq. (16.52) is treated as a random variable defined by the stochastic averages

$$
\ll \xi_{\alpha}(t) \gg=0, \quad \ll \xi_{\alpha}(t) \xi_{\beta}\left(t^{\prime}\right) \gg=2 D_{\alpha} \delta_{\alpha \beta} \delta\left(t-t^{\prime}\right),
$$

where $D_{\alpha}$ is a diffusion rate.

In this way, Eq. (16.44) transforms to the stochastic differential equation

$$
\frac{d^{2} \mathbf{r}}{d t^{2}}=\omega_{1}^{2}\left(S^{x} \mathbf{e}_{x}+S^{y} \mathbf{e}_{y}+\lambda S^{z} \mathbf{e}_{z}+\mathbf{G}\right)+\gamma \vec{\xi},
$$

and Eq. (16.45) is written in the form

$$
\frac{d \mathbf{S}}{d t}=\omega_{2} \hat{A} \mathbf{S}
$$

where $\hat{A}=\left[A_{\alpha \beta}\right]$ is an antisymmetric matrix with the elements

$$
\begin{gathered}
A_{\alpha \alpha}=0, \quad A_{\alpha \beta}=-A_{\beta \alpha}, \\
A_{12}=\alpha z, \quad A_{23}=x+h_{x}, \quad A_{31}=y+h_{y} .
\end{gathered}
$$


If one invokes for Eqs. (16.54) and (16.55) the adiabatic approximation, one finds an effective confining potential being harmonic near the trap center. In fact, the adiabatic approximation here assumes that the spin adiabatically follows the magnetic field, which implies that $d \mathbf{S} / d t=0$. The latter leads to the equality $\hat{A} \mathbf{S}=0$ or $\mathbf{B} \times \mathbf{S}=0$. Consequently, $\mathbf{S}$ is aligned along $\mathbf{B}$, so that one can put $\mathbf{S}=(\mathbf{S}(0) \cdot \mathbf{B}) \mathbf{B} / \mathbf{B}^{2}$. Substituting this in Eq. (16.54), one finds that the motion is approximately harmonic around the trap center. But recall that the adiabatic approximation has sense only for describing a stationary regime, when atoms are permanently trapped. And such an approximation is, in general, invalid for treating nonstationary regimes, e.g. when atoms fly out of the trap.

\subsection{Scale Separation Approach}

The evolution equations (16.54) and (16.55) can be treated by employing the Scale Separation Approach 409][412], which is a generalization of the averaging method [399] to the system of stochastic equations. To this end, it is necessary, first, to classify the functional variables onto fast and slow. The latter can be done by assuming the existence of the following small parameters

$$
\left|\frac{\omega_{1}}{\omega_{2}}\right| \ll 1, \quad\left|\frac{\omega}{\omega_{2}}\right| \ll 1, \quad\left|\frac{\gamma}{\omega_{2}}\right| \ll 1 .
$$

Then from Eqs. (16.54) and (16.55) it follows that $\mathbf{r}$ and $\mathbf{h}$ can be treated as slow, while $\mathbf{S}$ as fast. This permits one to solve Eq. (16.55) for the fast function, keeping the slow functions $\mathbf{r}$ and $\mathbf{h}$ as quasi-invariants, that is, the matrix $\hat{A}$ can also be kept as a quasi-invariant.

For the matrix $\hat{A}$, one can solve the eigenproblem

$$
\hat{A} \mathbf{b}_{j}=a_{j} \mathbf{b}_{j} \quad(j=1,2,3),
$$

obtaining the eigenvalues

$$
a_{1,2}= \pm i \sqrt{A_{12}^{2}+A_{23}^{2}+A_{31}^{2}}, \quad a_{3}=0
$$

and the eigenvectors

$$
\mathbf{b}_{j}=\frac{\left(A_{12} A_{23}-a_{j} A_{31}\right) \mathbf{e}_{x}+\left(A_{12} A_{31}+a_{j} A_{23}\right) \mathbf{e}_{y}+\left(A_{12}^{2}+a_{j}^{2}\right) \mathbf{e}_{z}}{\left[\left(A_{12}^{2}-\left|a_{j}\right|^{2}\right)^{2}+\left(A_{12}^{2}+\left|a_{j}\right|^{2}\right)\left(A_{23}^{2}+A_{31}^{2}\right)\right]^{1 / 2}} .
$$

The latter possess the properties

$$
\mathbf{b}_{1}^{*}=\mathbf{b}_{2}, \quad \mathbf{b}_{3}^{*}=\mathbf{b}_{3}, \quad \mathbf{b}_{1}^{2}=\mathbf{b}_{2}^{2}=0, \quad \mathbf{b}_{3}^{2}=1
$$

and they form an orthonormalized basis,

$$
\mathbf{b}_{i}^{*} \mathbf{b}_{j}=\delta_{i j}, \quad\left|\mathbf{b}_{i}\right|^{2}=1 .
$$

With the matrix $\hat{A}$ treated as a quasi-invariant, the solution to Eq. 16.55) reads

$$
\mathbf{S}(t)=\sum_{j=1}^{3} C_{j} \mathbf{b}_{j}(t) \exp \left\{\omega_{2} a_{j}(t) t\right\}
$$

where the coefficients

$$
C_{j}=\mathbf{S}(0) \cdot \mathbf{b}_{j}(0)
$$

are defined by initial conditions.

The solution (16.57) is to be substituted in the right-hand side of Eq. (16.54) for the slow variable, averaging this right-hand side over time and over the stochastic variable. In the process of the averaging, the functions $\mathbf{r}$ and $\mathbf{h}$ should be distinguished between each other due to the inequality

$$
\left|\frac{\omega_{1}}{\omega}\right| \ll 1
$$


that usually holds true. Then $\mathbf{r}$ is to be considered as slow, compared to the fast function $\mathbf{h}$. Thus, the double averaging procedure for a function $f(\mathbf{r}, \mathbf{h}, \vec{\xi}, t)$ is defined as

$$
\lim _{\tau \rightarrow \infty} \frac{1}{\tau} \int_{0}^{\tau} \ll f(\mathbf{r}, \mathbf{h}(t), \vec{\xi}, t) \gg d t,
$$

where the slow variable $\mathbf{r}$ is kept fixed. Accomplishing this procedure leads to the equation

$$
\frac{d^{2} \mathbf{r}}{d t^{2}}=\omega_{1}^{2}(\mathbf{F}+\mathbf{G})
$$

with the effective force

$$
\mathbf{F}=C_{3} \lim _{\tau \rightarrow \infty} \frac{1}{\tau} \int_{0}^{\tau}\left(b_{3}^{x} \mathbf{e}_{x}+b_{3}^{y} \mathbf{e}_{y}+\lambda b_{3}^{z} \mathbf{e}_{z}\right) d t,
$$

in which

$$
\begin{aligned}
& C_{3}=\frac{\left(x+h_{x}^{0}\right) S_{0}^{x}+\left(y+h_{y}^{0}\right) S_{0}^{y}+\lambda z S_{0}^{z}}{\left[\left(x+h_{x}^{0}\right)^{2}+\left(y+h_{y}^{0}\right)^{2}+\lambda^{2} z^{2}\right]^{1 / 2}}, \\
& \mathbf{b}_{3}=\frac{\left(x+h_{x}^{0}\right) \mathbf{e}_{x}+\left(y+h_{y}^{0}\right) \mathbf{e}_{y}+\lambda z \mathbf{e}_{z}}{\left[\left(x+h_{x}^{0}\right)^{2}+\left(y+h_{y}^{0}\right)^{2}+\lambda^{2} z^{2}\right]^{1 / 2}},
\end{aligned}
$$

where $h_{\alpha}^{0} \equiv h_{\alpha}(0)$ and $S_{0}^{\alpha} \equiv S^{\alpha}(0)$.

As an example of the transverse field (16.48) let us take the rotating field, used in some traps [85], when

$$
h_{x}=\cos \omega t, \quad h_{y}=\sin \omega t .
$$

Then the effective force (16.61) becomes

$$
\mathbf{F}=\frac{\left[(1+x) S_{0}^{x}+y S_{0}^{y}+\lambda z S_{0}^{z}\right]\left(x \mathbf{e}_{x}+y \mathbf{e}_{y}+2 \lambda^{2} z \mathbf{e}_{z}\right)}{2\left[\left(1+2 x+x^{2}+y^{2}+\lambda^{2} z^{2}\right)\left(1+x^{2}+y^{2}+\lambda^{2} z^{2}\right)\right]^{1 / 2}} .
$$

If initial conditions for the spin polarization are chosen so that $S_{0}^{x} \neq 0$ and $S_{0}^{y}=S_{0}^{z}=0$, then the force (16.63) at $|\mathbf{r}| \ll 1$ reduces to the harmonic form. For $S_{0}^{x}<0$, atoms are confined in the trap, oscillating in an effective harmonic potential. The presence of gravity does not change much this motion, simply shifting the equilibrium position from the trap center. This picture describes the standard motion of trapped atoms.

Suppose that, after atoms have been trapped, their spin polarization is prepared in the initial state

$$
S_{0}^{x}=S_{0}^{y}=0, \quad S_{0}^{z}=S .
$$

This can be done, for instance, by means of a short pulse of magnetic field. In quantum mechanics such a process is termed sudden perturbation [113, 414]. If the spin of trapped atoms was aligned along $\mathbf{B}_{2}$, then the duration of a magnetic pulse, turning spins to the polarization (16.64), has to be shorter than $\omega_{2}^{-1}$, and its amplitude larger than $B_{2}$. The initial spin polarization (16.64) is such that the spins are not aligned along the magnetic field B. Therefore, Eq. (16.64) corresponds to nonadiabatic initial conditions. Consequently, the following dynamics will also be nonadiabatic, and atoms will not be necessarily confined, but will escape from the trap. The finite size of the latter can be taken into account by introducing the trap shape factor

$$
\Xi(\mathbf{r}) \equiv 1-\Theta\left(x^{2}+y^{2}-R^{2}\right) \Theta\left(|z|-\frac{L}{2}\right),
$$

where the trap is assumed to have the shape of a cylinder of radius $R$ and length $L$, with $\Theta(\cdot)$ being a unit step function. Since the magnetic fields of the trap are supposed to act on atoms only inside the trap, the force (16.63), caused by these magnetic fields, should be nonzero only inside the trap. This is easy to take into account by multiplying (16.63) by the shape factor (16.65). Thus, the effective force of the trap magnetic fields, under the initial spin polarization (16.64), acquires the form

$$
\mathbf{F}=\frac{1}{2} \lambda S u z\left(x \mathbf{e}_{x}+y \mathbf{e}_{y}+2 \lambda^{2} z \mathbf{e}_{z}\right),
$$


in which $u=u(\mathbf{r})$,

$$
u(\mathbf{r}) \equiv \frac{\Xi(\mathbf{r})}{\left[\left(1+2 x+x^{2}+y^{2}+\lambda^{2} z^{2}\right)\left(1+x^{2}+y^{2}+\lambda^{2} z^{2}\right)\right]^{1 / 2}} .
$$

The evolution equation (16.60), with the effective force (16.66), possesses the property of invariance under the transformation

$$
\lambda S \rightarrow-\lambda S, \quad \mathbf{r} \rightarrow-\mathbf{r}, \quad \mathbf{G} \rightarrow-\mathbf{G} .
$$

Therefore, it is sufficient to consider the case of a fixed sign of $\lambda S$, say one can fix $\lambda S>0$.

For convenience, let us introduce the dimensionless gravitational force

$$
\boldsymbol{\delta} \equiv \frac{\mathbf{G}}{\lambda S}=\frac{\mathbf{g}}{\lambda S R_{0} \omega_{1}^{2}},
$$

and let us pass to the dimensionless time measured in units of $\left(\sqrt{\lambda S} \omega_{1}\right)^{-1}$. To return to the dimensional time, one has to make the replacement

$$
t \rightarrow \sqrt{\lambda S} \omega_{1} t .
$$

Then Eq. (16.60) yields

$$
\frac{d^{2} x}{d t^{2}}=\frac{1}{2} u z x+\delta_{x}, \quad \frac{d^{2} z}{d t^{2}}=\lambda^{2} u z^{2}+\delta_{z},
$$

where the equation for the $y$-component is not written down, being of the same form as the equation for the $x$-component.

Before analyzing Eqs. (16.70), it is useful to give estimates for the parameters typical of magnetic traps [6. 85]. The characteristic frequency of the atomic motion $\omega_{1} \sim 10^{2}-10^{3} \mathrm{~s}^{-1}$, the frequency of the spin motion $\omega_{2} \sim 10^{7}-10^{8} \mathrm{~s}^{-1}$. The frequency of the transverse rotating field is $\omega \sim 10^{4}-10^{5} \mathrm{~s}^{-1}$. The collision rate is $\gamma \sim 10 \mathrm{~s}^{-1}$. From here

$$
\frac{\omega_{1}}{\omega_{2}} \sim 10^{-5}, \quad \frac{\omega}{\omega_{2}} \sim 10^{-3}, \quad \frac{\gamma}{\omega_{2}} \sim 10^{-6}, \quad \frac{\omega_{1}}{\omega} \sim 10^{-2},
$$

which shows that the inequalities $(\mathbf{1 6 . 5 6})$ are valid, as well as the inequality (16.58). The characteristic length (16.49) is $R_{0} \sim 0.1-0.5 \mathrm{~cm}$. For $S \sim 1$ and the gravitational acceleration $g \sim 10^{3} \mathrm{~cm} / \mathrm{s}^{2}$, by choosing appropriate $\lambda$ and $\omega_{1}$, one can always make the dimensionless gravitational force $(16.69)$ small, so that $\left|\delta_{\alpha}\right| \ll 1$. Thus, for the typical values $\lambda \sim 2, S \sim 1, R_{0} \sim 0.5 \mathrm{~cm}$ and $\omega_{1} \sim 10^{2}-10^{3} \mathrm{~s}^{-1}$, one has $\delta_{\alpha} \sim 10^{-3}-10^{-1}$.

\subsection{Magnetic Semiconfinement of Atoms}

The evolution equations $(16.70$ ) possess solutions displaying an interesting regime of semiconfined motion, when atoms are confined from one side of the axis $z$ but are not confined from another side [408], [415]-419]. This semiconfinement is realized by means of only magnetic fields, without involving additional laser beams kicking atoms out and without mechanical collimators. The existence of such a magnetic semiconfinement can be demonstrated both analytically and numerically.

First, let us demonstrate the occurrence of semiconfinement analytically. Since $\left|\delta_{\alpha}\right| \ll 1$, the presence of gravity does not drastically shift the center of the atomic cloud from the trap center. So that for atoms in the middle of the trap one can put $|\mathbf{r}| \ll 1$. Then the function (16.67) reduces to

$$
u(\mathbf{r}) \simeq 1 \quad(|\mathbf{r}| \ll 1) .
$$

Using this, the second of Eqs. (16.70) can be transformed to the form

$$
\left(\frac{d z}{d t}\right)^{2}=\frac{2}{3} \lambda^{2}\left(z^{3}-z_{0}^{3}\right)+2 \delta_{z}\left(z-z_{0}\right)+\dot{z}_{0}^{2},
$$


where $z_{0}=z(0)$ and $\dot{z}_{0}=\dot{z}(0)$ are initial conditions for the location and velocity in the $z$-direction, respectively. With the notation

$$
z(t)=\frac{6}{\lambda^{2}} \mathcal{P}\left(t-t_{0}\right),
$$

in which $t_{0}$ is an integration constant defined by the initial condition

$$
z(0)=\frac{6}{\lambda^{2}} \mathcal{P}\left(-t_{0}\right)=z_{0},
$$

the form (16.72) reduces to the Weierstrass equation

$$
\left(\frac{d \mathcal{P}}{d t}\right)^{2}=4 \mathcal{P}^{3}-g_{2} \mathcal{P}-g_{3}
$$

with the invariants

$$
g_{2} \equiv-\frac{1}{3} \lambda^{2} \delta_{z}, \quad g_{3} \equiv \frac{\lambda^{4}}{54}\left(\lambda^{2} z_{0}^{3}+3 \delta_{z} z_{0}-\frac{3}{2} \dot{z}_{0}^{2}\right) .
$$

The solution to Eq. (16.74) is called the elliptic Weierstrass function [420]. To analyze possible regimes of motion, it is useful to introduce the characteristic roots $e_{i}(i=1,2,3)$ defined by the equation

$$
4 e_{i}^{3}-g_{2} e_{i}-g_{3}=0,
$$

whose solutions are

$$
\begin{gathered}
e_{1,2}=-\frac{1}{4}\left(C+\frac{g_{2}}{3 C}\right) \pm \frac{\sqrt{3}}{4} i\left(C-\frac{g_{2}}{3 C}\right), \\
e_{3}=\frac{1}{2}\left(C+\frac{g_{2}}{3 C}\right), \quad C^{3} \equiv g_{3}+\sqrt{g_{3}^{2}-\frac{g_{2}^{3}}{27}} .
\end{gathered}
$$

Then Eq. (16.74) can be written as

$$
\left(\frac{d \mathcal{P}}{d t}\right)^{2}=4\left(\mathcal{P}-e_{1}\right)\left(\mathcal{P}-e_{2}\right)\left(\mathcal{P}-e_{3}\right) .
$$

The properties of the characteristic roots (16.76) depend on the sign of the determinant $g_{2}^{3} / 27-g_{3}^{2}$. There are three different cases:

When $g_{2}^{3}<27 g_{3}^{2}$, the roots $e_{1}$ and $e_{2}$ are complex conjugate, while $e_{3}$ is real. The right-hand side of Eq. (16.77) can be presented as $4\left|\mathcal{P}-e_{1}\right|^{2}\left(\mathcal{P}-e_{3}\right)$. As far as the left-hand side of Eq. (16.77) is nonnegative, it follows that $\mathcal{P} \geq e_{3}$ or, according to the relation (16.73), one gets $z \geq 6 e_{3} / \lambda^{2}$.

If $g_{2}^{3}=27 g_{3}^{2}$, then all three characteristic roots are real and are

$$
e_{1}=e_{2}=-\frac{1}{2} g_{3}^{1 / 3}, \quad e_{3}=g_{3}^{1 / 3}
$$

Again, admissible solutions are to be such that the right-hand side of Eq. (16.77) be nonnegative. This gives, as in the previous case, $\mathcal{P} \geq e_{3}$, hence $z \geq 6 e_{3} / \lambda^{2}$. Thus, in both cases considered, one has $z \geq z_{\text {min }}$, with

$$
z_{\min } \equiv \frac{6}{\lambda^{2}} e_{3} .
$$

That is, the motion along the $z$-axis is confined from below by the minimal value (16.78), but it is not confined from above. This means nothing but semiconfined motion. Such a type of motion is realized for $g_{2}^{3} \leq 27 g_{3}^{2}$, which yields the inequality

$$
-\delta_{z}^{3} \leq \frac{\lambda^{2}}{4}\left(\lambda^{2} z_{0}^{3}+3 \delta_{z} z_{0}-\frac{3}{2} \dot{z}_{0}^{2}\right)^{2}
$$


The latter would always hold true if there were no gravity or when the trap axis is directed along the gravitational force, that is when $\delta_{z} \geq 0$. However, if $\delta_{z}<0$, this inequality is valid not for all initial conditions, though for the majority of them, since $\left|\delta_{z}\right| \ll 1$.

For the case $g_{2}^{3}>27 g_{3}^{2}$, which is possible only for $g_{2} \geq 0$, hence $\delta_{z} \leq 0$, the characteristic roots 16.76 ) are real and can be written as

$$
\begin{gathered}
e_{1,2}=-\frac{1}{2} \sqrt{\frac{g_{2}}{3}}\left(\cos \frac{\varphi}{3} \pm \sqrt{3} \sin \frac{\varphi}{3}\right), \\
e_{3}=\sqrt{\frac{g_{2}}{3}} \cos \frac{\varphi}{3}, \quad \varphi \equiv \operatorname{arctg}\left(\frac{g_{2}^{3}}{27 g_{3}^{2}}-1\right)^{1 / 2} .
\end{gathered}
$$

The roots are arranged so that $e_{1}<e_{2} \leq 0<e_{3}$. There are two admissible kinds of motion. One kind corresponds to $z \geq z_{\text {min }}$, with the same minimal $z$ as in Eq. (16.78), which is again the semiconfined motion. And the other type corresponds to a motion confined between $e_{1}$ and $e_{2}$, so that $e_{1} \leq z \leq e_{2}$. This means that in the whole phase space of initial conditions, the fraction of atoms that remain confined is of order $e_{2}-e_{1}$, while all other atoms are semiconfined.

To estimate the fraction of atoms that remain confined, one can take into account that $\left|\delta_{\alpha}\right| \ll 1$, thence $g_{2} \ll 1$, which shows that $g_{3} \simeq 0$. From here $\varphi \simeq \pi / 2$, and the related characteristic roots are

$$
e_{1} \simeq-\frac{\sqrt{g_{2}}}{2}, \quad e_{2} \simeq 0, \quad e_{3} \simeq \frac{\sqrt{g_{2}}}{2} .
$$

This results in

$$
e_{2}-e_{1}=\frac{\sqrt{g_{2}}}{2}=\frac{\lambda}{2 \sqrt{3}}\left|\delta_{z}\right|^{1 / 2}
$$

which for the typical values of the parameters considered above gives $e_{2}-e_{1} \sim 10^{-2}-10^{-1}$. Therefore the fraction of atoms that remain confined is less than $10 \%$ and can be made as small as $1 \%$.

The elliptic Weierstrass function, being the solution of Eq. (16.74), diverges at $t \rightarrow t_{0}$, which results in the divergence of the $z$-variable (16.73) according to the law

$$
z(t) \rightarrow \frac{6}{\lambda^{2}\left|t-t_{0}\right|^{2}} \quad\left(t \rightarrow t_{0}\right) .
$$

The characteristic time

$$
t_{0}=\int_{p_{0}}^{\infty} \frac{d p}{\sqrt{4 p^{3}-g_{2} p-g_{3}}} \quad\left(p_{0} \equiv \frac{\lambda^{2}}{6} z_{0}\right)
$$

can serve as an estimate for the escape time, that is the time after which an atom, starting at the location $z_{0}$ at $t=0$, leaves the trap. The estimates for the typical trap parameters give 408 an escape time of order $0.1 \mathrm{~s}$.

The existence of semiconfinement has been confirmed 416, 417, 419 by direct numerical solution of Eqs. (16.70). Several typical trajectories for the cross-section $x(t)-z(t)$ and the related phase portraits for the velocities $\dot{x}(t)$ and $\dot{z}(t)$ are presented in Figs. 41 to 42, for the trap axis inclined by the 45 degrees to the horizon. The influence of gravity, as is seen, results in curving the trajectories, similarly to what happens to cannon shells. Stronger gravitational force bends the trajectories stronger. But the semiconfining regime remains.

To consider the role of random collisions, described by the term $\gamma \vec{\xi}$ in Eq. (16.54), one has, after substituting the fast solution (16.57) into Eq. (16.54), to average the right-hand side of the latter over time, as in the definition (16.59), but without averaging over the variable $\vec{\xi}$. The rotating transverse field (16.62), initial spin polarization (16.64), trap shape factor (16.65), and all notations are assumed to be as before. Then, instead of Eqs. (16.70), one obtains

$$
\frac{d^{2} x}{d t^{2}}=\frac{1}{2} u z x+\delta_{x}+\frac{\gamma}{\lambda S \omega_{1}^{2}} \xi_{x}, \quad \frac{d^{2} z}{d t^{2}}=\lambda^{2} u z^{2}+\delta_{z}+\frac{\gamma}{\lambda S \omega_{1}^{2}} \xi_{z} .
$$

The random variables $\xi_{\alpha}$ are characterized by the stochastic averages (16.53), the second of which, for the dimensionless time used in Eq. (16.80), writes

$$
\ll \xi_{\alpha}(t) \xi_{\beta}\left(t^{\prime}\right) \gg=2 D_{\alpha} \delta_{\alpha \beta} \sqrt{\lambda S} \omega_{1} \delta\left(t-t^{\prime}\right),
$$


just because the time here is measured in units of $\left(\sqrt{\lambda S} \omega_{1}\right)^{-1}$.

If the main behavior of the system were governed by intensive random collisions, then, as is evident, no ordered semiconfining regime of motion could exist. The disorganized chaotic motion of atoms is of no interest for the present investigation. What is important is to find conditions under which random collisions would not much disturb the semiconfinement of atoms. Therefore the terms in Eqs. (16.80), which are related to random collisions, can be treated as weak perturbations. To this end, the solutions to Eqs. (16.80) may be presented as the sums

$$
x=x_{1}+x_{2}, \quad z=z_{1}+z_{2},
$$

in which $x_{1}$ and $z_{1}$ are the solutions to Eq. (16.70), while $x_{2}$ and $z_{2}$ are given by the linearized equations. The latter, for $|\mathbf{r}| \ll 1$, when $u(\mathbf{r}) \approx 1$, are

$$
\frac{d^{2} x_{2}}{d t^{2}}=\frac{1}{2}\left(z_{1} x_{2}+x_{1} z_{2}\right)+\frac{\gamma}{\lambda S \omega_{1}^{2}} \xi_{x}, \quad \frac{d^{2} z_{2}}{d t^{2}}=2 \lambda^{2} z_{1} z_{2}+\frac{\gamma}{\lambda S \omega_{1}^{2}} \xi_{z} .
$$

As earlier, the equation for the $y$-component is not written down, since it has the same form as that for the $x$-component. The solutions to Eqs. (16.82) can be presented as

$$
\begin{gathered}
x_{2}(t)=\int_{0}^{t} G_{x}\left(t-t^{\prime}\right)\left[\frac{\gamma}{\lambda S \omega_{1}^{2}} \xi_{x}\left(t^{\prime}\right)+\frac{1}{2} x_{1} z_{2}\left(t^{\prime}\right)\right] d t^{\prime}, \\
z_{2}(t)=\int_{0}^{t} G_{z}\left(t-t^{\prime}\right) \frac{\gamma}{\lambda S \omega_{1}^{2}} \xi_{z}\left(t^{\prime}\right) d t^{\prime},
\end{gathered}
$$

where

$$
G_{\alpha}(t) \equiv \frac{\sinh \left(\varepsilon_{\alpha} t\right)}{\varepsilon_{\alpha}}, \quad \varepsilon_{x}^{2} \equiv \frac{1}{2} z_{1}, \quad \varepsilon_{z}^{2} \equiv 2 \lambda^{2} z_{1} .
$$

According to the properties of the random variables $\xi_{\alpha}$, one has

$$
\ll x_{2} \gg=\ll z_{2} \gg=0 \text {. }
$$

Calculating the mean-square deviations, one can treat $x_{1}$ and $z_{1}$ as slow variables, keeping them as quasiinvariants. Then one obtains

$$
\begin{gathered}
\ll x^{2} \gg=\frac{\gamma^{2} D_{x} t}{(\lambda S)^{3 / 2} \omega_{1}^{3} \varepsilon_{x}^{2}}\left[\frac{\sinh \left(2 \varepsilon_{x} t\right)}{2 \varepsilon_{x} t}-1\right]+\frac{\gamma^{2} D_{x} x_{1}^{2} \varepsilon_{x}^{4} t}{(\lambda S)^{3 / 2} \omega_{1}^{3} z_{1}^{2} \varepsilon_{z}^{2}\left(\varepsilon_{z}^{2}-\varepsilon_{x}^{2}\right)} \times \\
\times\left\{\frac{\sinh \left(\varepsilon_{z} t\right)}{\varepsilon_{z} t}\left[\cosh \left(\varepsilon_{z} t\right)-\cosh \left(\varepsilon_{x} t\right)\right]-\frac{\varepsilon_{z}}{\varepsilon_{x}} \sinh \left(\varepsilon_{z} t\right) \sinh \left(\varepsilon_{x} t\right)+\cosh \left(\varepsilon_{z} t\right) \cosh \left(\varepsilon_{x} t\right)-1\right\}, \\
\ll z^{2} \gg=\frac{\gamma^{2} D_{z} t}{(\lambda S)^{3 / 2} \omega_{1}^{2} \varepsilon_{z}^{2}}\left[\frac{\sinh \left(2 \varepsilon_{z} t\right)}{2 \varepsilon_{z} t}-1\right] .
\end{gathered}
$$

These solutions show that the small parameter here is

$$
\frac{\gamma^{2} D}{(\lambda S)^{3 / 2} \omega_{1}^{3}} \ll 1, \quad D \equiv \sup _{\alpha}\left\{D_{\alpha}\right\} .
$$

Under the inequality (16.84), random collisions can be considered as a weak perturbation not essentially disturbing the semiconfined motion of atoms. Taking, for estimates, the collision rate as $\gamma \sim \hbar \rho a_{s} / m_{0}$, where $\rho$ is the density of atoms, and the diffusion rate as $D \sim k_{B} T / \hbar$, where $T$ is temperature, one gets from Eq. (16.84) the condition

$$
\frac{k_{B} T \hbar \rho^{2} a_{s}^{2}}{m_{0}^{2}(\lambda S)^{3 / 2} \omega_{1}^{3}} \ll 1 \text {. }
$$

If one takes the parameters typical of experiments with ${ }^{87} \mathrm{Rb}$ and ${ }^{23} \mathrm{Na}$, that is $m_{0} \sim 10^{-22} \mathrm{~g}, a_{s} \sim 5 \times 10^{-7} \mathrm{~cm}$, $\omega_{1} \sim 10^{3} \mathrm{~s}^{-1}, \lambda S \sim 1$, and $\rho \sim 10^{12}-10^{14} \mathrm{~cm}^{-3}$, then the condition (16.85) requires $T \ll T_{0} \sim 10^{-5}-10^{-1} \mathrm{~K}$. 
Such temperatures are essentially higher than the Bose-condensation temperatures for the corresponding atoms. Hence, the Bose-condensed trapped atoms can be coupled out of the trap in the regime of semiconfined motion. This mechanism can be employed for creating well-collimated beams from atom lasers in arbitrary direction. Such highly-directional beams can be formed by means of only magnetic fields. That is why the described effect has been named the magnetic semiconfinement of atoms [408], 415]-419]. 


\section{Chapter 17}

\section{Bose-Einstein Condensate in Liquids}

In the previous chapters, Bose-Einstein condensate in trapped atomic gases has been considered. Similar types of condensates can appear in other gases with sufficiently weak interparticle interaction. For example, BoseEinstein condensation of excitons in $\mathrm{CuCl}$ and $\mathrm{Cu}_{2} \mathrm{O}$ has been studied both theoretically and experimentally 421-423. It has been predicted 288, 289 that in dense nuclear matter the Bose condensation of dibaryons can happen 4424- 426], which suggests the possibility of creating dibaryon lasers 427 .

As has been demonstrated by Bogolubov [282], Bose-Einstein condensation does generally occur in weakly nonideal Bose gases. But an important question is whether the condensation remains in Bose liquids, that is in the systems of strongly interacting atoms. The most known and intensively studied such liquid is superfluid ${ }^{4}$ He. Since London [428] and Tisza [429], it is commonly believed that superfluidity in helium is somehow connected to Bose condensation, although an explicit relation between the superfluid and condensate fraction is unknown till nowadays. In this Chapter, we shall briefly touch some problems in the theoretical description of strongly interacting systems and will discuss the most accurate experiments aiming at measuring the condensate fraction in superfluid helium. It is not our goal to give here a detailed review of these topics which voluminous literature is devoted to, but we shall sketch only some, to our mind, most interesting points, paying attention to differences and similarities in the features of liquids and gases.

\subsection{Differences between Liquids and Gases}

There are several important differences that are immediately noticeable when comparing liquids with gases. For concreteness, liquid ${ }^{4} \mathrm{He}$ at saturated vapor pressure will be considered in what follows. With the density $\rho=0.0218 \AA^{-3}$, the mean interatomic distance is $a=3.58 \AA$. The superfluid transition temperature is $T_{c}=2.17$ $\mathrm{K}$. For this temperature and mass $m_{0}=6.64 \times 10^{-24} \mathrm{~g}$, the thermal wavelength is $\lambda_{T}=5.93 \AA$.

Here, one may notice the first difference, making it clear that at $T_{c}$ the ratio $a / \lambda_{T}=0.6$ is not much less than one, so that inequality (7.1) is not valid. Respectively, $\rho \lambda_{T}^{3}=4.6$. This, however, does not look yet too dangerous, since by lowering temperature, one always can reach the point when $\lambda_{T} \gg a$.

The more warning sign is that inequality (7.2) never holds true. To make this transparent, one needs to define the interaction radius. For an interaction potential $\Phi(r)$, with a hard core of radius $\sigma$, the interaction radius is defined as

$$
r_{i n t} \equiv \sigma+\frac{\int_{\sigma}^{\infty} r \Phi(r) r^{2} d r}{\int_{\sigma}^{\infty} \Phi(r) r^{2} d r}
$$

In the case of soft-core potentials, one can put $\sigma \rightarrow 0$. But the interaction for helium atoms is usually described by hard-core potentials.

The most popular is the Lennard-Jones potential

$$
\Phi(r)=4 \varepsilon\left[\left(\frac{\sigma}{r}\right)^{12}-\left(\frac{\sigma}{r}\right)^{6}\right]
$$


in which

$$
\varepsilon=10.22 \mathrm{~K} \quad \sigma=2.556 \AA .
$$

Here, $\varepsilon$ is given in the Kelvin scale. The minimum of this potential, given by the expression

$$
r_{m}=2^{1 / 6} \sigma=2.87 \AA, \quad \Phi\left(r_{m}\right)=-\varepsilon,
$$

is located at the point that is smaller than the interatomic distance $a$. There exist also several other potentials [430. One of the best representations of the helium interaction is produced by the Aziz potential 431. 433] having the form

$$
\Phi(r)=\varepsilon\left[A e^{-\alpha x-\beta x^{2}}-F(x)\left(\frac{c_{6}}{x^{6}}+\frac{c_{8}}{x^{8}}+\frac{c_{10}}{x^{10}}\right)\right],
$$

in which

$$
F(x)=\left\{\begin{array}{cl}
\exp \left[-\left(\frac{\delta}{x}-1\right)^{2}\right], & x \leq \delta \\
1, & x \geq \delta
\end{array}\right.
$$

and the dimensionless variable

$$
x \equiv \frac{r}{r_{m}}, \quad \Phi\left(r_{m}\right)=-\varepsilon,
$$

defines the radius normalized to the point of minimum $r_{m}$, so that

$$
\varepsilon=10.94 \mathrm{~K}, \quad r_{m}=2.97 \AA .
$$

The other parameters, according to the last version [433], are

$$
\begin{gathered}
A=1.922 \times 10^{5}, \quad \alpha=10.735, \quad \beta=1.893, \quad \delta=1.414, \\
c_{6}=1.349, \quad c_{8}=0.414, \quad c_{10}=0.171 .
\end{gathered}
$$

Calculating the interaction radius (17.1) is more convenient for the simpler Lennard-Jones potential (17.2). This gives $r_{i n t}=2.69 \sigma=6.88 \AA$. Comparing it with the interatomic distance, one has $r_{i n t} / a=1.9$, from where $\rho r_{i n t}^{3}=7.1$. Hence, Eq. (7.2) is not valid, as well as the second of Eqs. (7.3), since

$$
\rho r_{i n t}^{3} \gg 1 \text {. }
$$

Therefore, the condensate in a liquid, where Eq. (17.4) holds true, should be rather depleted, if condensation can occur at all.

The third peculiarity results from the fact that inequality (17.4) contradicts Eqs. (9.2), because of which one cannot simplify the consideration resorting to the cartoon potential (9.3), but one is doomed to operate with the full potentials like those above.

One more problem immediately arises from the previous, due to the sad circumstance that the hard-core potentials, as the Lennard-Jones one, are not integrable, i.e. they do not satisfy condition (7.30). Because of this, it is impossible to break gauge symmetry by means of the Bogolubov prescription, as is discussed in section 7.4. Nontrivial coherent states also do not exist for nonintegrable potentials, as is explained in Chapter 8. Thus, atoms cannot be in pure coherent states, but can be only partially coherent. To cope with the nonintegrability of the interaction potentials, one has to accurately take into account interatomic correlations, especially short-range ones. For this purpose, without breaking gauge symmetry, one employs 434- 437 the Jastrow-type variational functions

$$
\Psi\left(\mathbf{r}_{1}, \mathbf{r}_{2}, \ldots, \mathbf{r}_{N}\right)=\prod_{i<j} f\left(r_{i j}\right) \prod_{i<j<k} f_{3}\left(r_{i j}, r_{j k}, r_{k i}\right),
$$

in which $r_{i j} \equiv\left|\mathbf{r}_{i}-\mathbf{r}_{j}\right| ; f(\cdot)$ is a pair correlation function, and $f_{3}(\cdot)$ is a triplet correlation function. The pair correlation function behaves, at short distance, as

$$
f(r) \sim \exp \left\{-\frac{1}{2}\left(\frac{b}{r}\right)^{5}\right\} \quad(r \rightarrow 0)
$$


and at large distance, it has the asymptotic behavior

$$
f(r) \simeq 1-\left(\frac{m c}{2 \pi^{2} \hbar \rho}\right) \frac{1}{r^{2}} \quad(r \rightarrow \infty),
$$

where $c$ is the velocity of sound. Exponentially tending to zero as $r \rightarrow 0$, the correlation function (17.6) smooths the divergence of the interaction potential making the smoothed potential

$$
\bar{\Phi}(r) \equiv f(r) \Phi(r)
$$

integrable. Note that, although the Aziz potential (17.3) is formally finite at $r=0$, its value $\Phi(0) \sim 10^{6} \mathrm{~K}$ is so large that this potential is also to be considered as a hard-core potential, necessarily needing to take into account interatomic correlations smoothing its sharp rise at $r=0$. The smoothing radius $b$ in Eq. (17.6) can be treated as a variational parameter or can be determined from the Schrödinger equation for a pair wave function [438]- 440], from where

$$
b=\left(\frac{4}{5 \Lambda}\right)^{1 / 5} \sigma
$$

here the DeBoer parameter

$$
\Lambda \equiv \frac{\hbar}{\sigma \sqrt{m_{0} k_{B} \varepsilon}} .
$$

For helium, $\Lambda=0.426$ and $b=1.13 \sigma=2.89 \AA$. Correlation functions can also be found by invoking a cumulenttype expansion in the frame of the method of collective variables [41]-443]. These functions can be optimized by solving the Euler-Lagrange equations [434- 436]. The large-distance behavior of the correlation function (17.7) is a consequence of the existence of long-wavelength phonons [444]. After the smoothed potential (17.8) is defined, it is possible to develop a systematic iterative procedure for Green function equations [291, 355].

The necessity of taking account of strong interatomic correlations at the very first step of any iterative procedure is dictated by two reasons. One, as is explained above, is the nonintegrability of the hard-core interaction potentials, because of which the Fourier transforms of such potentials do not exist. Another reason is that the application of simple perturbation theory, without an appropriate account of correlations, can lead to senseless results. As an example, we may try to calculate, by using perturbation theory [445], the density of Bose-condensed atoms at zero temperature, which yields

$$
\rho_{0}=\rho-\frac{1}{3 \pi^{2} \hbar^{3}}\left[\rho m_{0} \tilde{\Phi}(0)\right]^{3 / 2},
$$

where $\tilde{\Phi}(k)$ is the Fourier transform of the interaction potential. As is said above, such a transform does not exist for nonintegrable potentials. But even assuming a soft-core potential, one has $\tilde{\Phi}(0) \approx k_{B} \varepsilon / \rho$, which for helium results in $\rho_{0} / \rho \approx-0.16$, that is a physically senseless negative value for the condensate density.

The impossibility of applying simple perturbation theory to liquids can be easily understood remembering that for this theory to be applicable requires the smallness of the ratio of the mean potential energy to the mean kinetic energy. However, for liquids, this ratio is never small, but, on the contrary, it is usually larger than one. For helium, as follows from theoretical calculations [443, 446, 447] and experiments 4448] -451], this ratio is about two.

\subsection{Definition of Superfluid Density}

One commonly believes that superfluidity appears simultaneously with Bose condensation, although the relation between the superfluid and condensate fractions has never been established for liquids. Thus, at zero temperature, all volume of helium is superfluid, while the condensate fraction does not exceed a value of about $10 \%$, and no general relation between these fractions is known.

The condensate density is defined as the difference

$$
\rho_{0}=\rho-\tilde{\rho}
$$


between the total density $\rho$ and the density of noncondensed atoms,

$$
\tilde{\rho} \equiv \frac{1}{(2 \pi)^{3}} \int n(k) d \mathbf{k}, \quad n(k) \equiv<a_{k}^{\dagger} a_{k}>,
$$

in which $n(k)$ is the momentum distribution.

The superfluid density can be determined by analyzing the response of the fluid to the motion imposed by boundary conditions 445, 452, 453. For this purpose, one needs to study what happens when the system is subject to an external perturbation, such that the liquid starts moving uniformly with velocity $\mathbf{v}$. This motion could be achieved by pushing the liquid through a tube having a pressure difference at its ends or enclosing the system between two rotating cylinders of radii much larger than the distance between the cylinder walls.

For a system uniformly moving with velocity $\mathbf{v}$, the field operator in the laboratory frame, $\psi_{v}$, is connected with the field operator $\psi$ in the frame, where the system is immovable, through the Galilean transformation

$$
\psi_{v}(\mathbf{r})=\psi(\mathbf{r}) \exp \left(i \frac{m_{0}}{\hbar} \mathbf{v} \cdot \mathbf{r}\right) .
$$

Then the operators of observables in the laboratory frame are obtained by taking $\psi_{v}$ instead of $\psi$. For instance, the Hamiltonian (8.13) becomes

$$
H_{v}=H+\int \psi^{\dagger}(\mathbf{r})\left(\mathbf{v} \cdot \hat{\mathbf{p}}+\frac{1}{2} m_{0} v^{2}\right) \psi(\mathbf{r}) d \mathbf{r},
$$

where $\hat{\mathbf{p}} \equiv-i \hbar \boldsymbol{\nabla}$. The number-of-atoms operator does not change,

$$
\hat{N}_{v}=\hat{N}=\int \psi^{\dagger}(\mathbf{r}) \psi(\mathbf{r}) d \mathbf{r} .
$$

And the momentum operator

$$
\hat{\mathbf{P}} \equiv \int \psi^{\dagger}(\mathbf{r}) \hat{\mathbf{p}} \psi(\mathbf{r}) d \mathbf{r}
$$

transforms to

$$
\hat{\mathbf{P}}_{v}=\hat{\mathbf{P}}+m_{0} N \mathbf{v} .
$$

Observable quantities from the algebra of observables $\mathcal{A}$ are given by the average

$$
<\mathcal{A}>_{v} \equiv \operatorname{Tr} \hat{\rho}_{v} \mathcal{A}
$$

with the statistical operator

$$
\hat{\rho}_{v} \equiv \frac{\exp \left\{-\beta\left(H_{v}-\mu \hat{N}\right)\right\}}{\operatorname{Tr} \exp \left\{-\beta\left(H_{v}-\mu \hat{N}\right)\right\}},
$$

where $\beta \equiv\left(k_{B} T\right)^{-1}$. For the momentum operator (17.16), one gets

$$
<\hat{\mathbf{P}}_{v}>_{v}=<\hat{\mathbf{P}}>_{v}+m_{0} N \mathbf{v} .
$$

The part of the liquid, which nontrivially responds to the perturbative motion, defines the superfluid component with the density

$$
\rho_{s} \equiv \frac{\rho}{m_{0} N} \lim _{\mathbf{v} \rightarrow 0} \frac{\partial}{\partial v^{\alpha}}<\hat{P}_{v}^{\alpha}>_{v} .
$$

Here, it is taken into account that, for an initially isotropic system, the density (17.20) should not depend on the direction of the probing velocity, that is, $\rho_{s}$ does not depend on the index $\alpha$.

To calculate the superfluid density (17.20), one has to analyze the limit $\mathbf{v} \rightarrow 0$. In this limit, linearizing the statistical operator (17.18), one finds

$$
\hat{\rho}_{v} \simeq \hat{\rho}[1+\beta \mathbf{v} \cdot(<\hat{\mathbf{P}}>-\hat{\mathbf{P}})],
$$


which for the average (17.17) yields

$$
<\mathcal{A}>_{v} \simeq<\mathcal{A}>+\beta \mathbf{v} \cdot(<\hat{\mathbf{P}}><\mathcal{A}>-<\hat{\mathbf{P}} \mathcal{A}>) .
$$

Here, $\langle\mathcal{A}>\equiv \operatorname{Tr} \rho \mathcal{A}$ implies an average in the frame at rest.

For an isotropic system, one has

$$
<\hat{\mathbf{P}}>=\hbar \sum_{k} \mathbf{k} n(k)=0 .
$$

Because of this, the statistical operator 17.21) simplifies as

$$
\hat{\rho}_{v} \simeq \hat{\rho}(1-\beta \mathbf{v} \cdot \hat{\mathbf{P}})
$$

and the average (17.22) reduces to

$$
<\mathcal{A}>_{v} \simeq<\mathcal{A}>-\beta \mathbf{v} \cdot\langle\hat{\mathbf{P}} \mathcal{A}>
$$

For example,

$$
<\hat{\mathbf{P}}>_{v} \simeq-\beta<(\mathbf{v} \cdot \hat{\mathbf{P}}) \hat{\mathbf{P}}>.
$$

Using this, for the average (17.19) one obtains

$$
<\hat{\mathbf{P}}_{v}>_{v} \simeq m_{0} N \mathbf{v}-\beta<(\mathbf{v} \cdot \hat{\mathbf{P}}) \hat{\mathbf{P}}>.
$$

In the case of an isotropic system, one can employ the equality

$$
<\hat{P}^{\alpha} \hat{P}^{\beta}>=\delta_{\alpha \beta}<\left(\hat{P}^{\alpha}\right)^{2}>=\frac{1}{3} \delta_{\alpha \beta}<\hat{\mathbf{P}}^{2}>,
$$

which gives

$$
\frac{\partial}{\partial v_{\alpha}}<\hat{P}_{v}^{\alpha}>_{v} \simeq m_{0} N-\frac{\beta}{3}<\hat{\mathbf{P}}^{2}>.
$$

Finally, the superfluid density $(17.20)$ takes the form

$$
\rho_{s}=\rho-\frac{\rho \beta}{3 m_{0} N}<\hat{\mathbf{P}}^{2}>.
$$

Since one also has the relation

$$
\rho_{s}=\rho-\rho_{n},
$$

where $\rho_{n}$ is the density of the normal component, the comparison of Eqs. (17.28) and (17.29) yields

$$
\rho_{n}=\frac{\rho \beta}{3 m_{0} N}<\hat{\mathbf{P}}^{2}>.
$$

This tells that the normal component is related to the dissipated energy of motion, while the superfluid component corresponds to nondissipative motion.

The dissipative term $<\hat{\mathbf{P}}^{2}>$ can be written in several forms. It can be expressed through the momentummomentum correlation function as

$$
<\hat{\mathbf{P}}^{2}>=\int<\hat{\mathbf{p}}(\mathbf{r}) \hat{\mathbf{p}}\left(\mathbf{r}^{\prime}\right)>d \mathbf{r} d \mathbf{r}^{\prime},
$$

where the momentum-density operator is

$$
\hat{\mathbf{p}}(\mathbf{r}) \equiv \psi^{\dagger}(\mathbf{r})(-i \hbar \nabla) \psi(\mathbf{r}) .
$$


It can also be connected with the two-particle Green function

$$
G_{2}(1234) \equiv-<\hat{T} \psi(1) \psi(2) \psi^{\dagger}(3) \psi^{\dagger}(4)>,
$$

in which $\hat{T}$ is the time-ordering operator and $\psi(j) \equiv \psi\left(\mathbf{r}_{j}, t_{j}\right)$. One has

$$
<\hat{\mathbf{P}}^{2}>=\hbar^{2} \int \lim _{3142}\left(\nabla_{1} \cdot \nabla_{2}\right) G_{2}(1234) d \mathbf{r}_{1} d \mathbf{r}_{2},
$$

where the limit means

$$
\lim _{3142} \equiv \lim _{\mathbf{r}_{3} \rightarrow \mathbf{r}_{1}} \lim _{\mathbf{r}_{4} \rightarrow \mathbf{r}_{2}} \lim _{t_{i} \rightarrow t}
$$

under the condition $t_{3}>t_{1}>t_{4}>t_{2}$. Passing to the momentum representation by means of the Fourier transform

$$
\psi(\mathbf{r})=\frac{1}{\sqrt{V}} \sum_{k} a_{k} e^{i \mathbf{k} \cdot \mathbf{r}},
$$

one gets

$$
<\hat{\mathbf{P}}^{2}>=\hbar^{2} \sum_{k k^{\prime}}\left(\mathbf{k} \cdot \mathbf{k}^{\prime}\right)<a_{k}^{\dagger} a_{k} a_{k^{\prime}}^{\dagger} a_{k^{\prime}}>.
$$

To find explicit expressions for the condensate density (17.11) and the superfluid density (17.28), one needs to specify the problem. It is straightforward to show how to accomplish calculations for the ideal gas with the Hamiltonian

$$
H=\sum_{k}\left(\hbar \omega_{k}-\mu\right) a_{k}^{\dagger} a_{k},
$$

where $\omega_{k}$ is a particle spectrum and $\mu$, the chemical potential. Then one has

$$
<a_{k}^{\dagger} a_{k} a_{k^{\prime}}^{\dagger} a_{k^{\prime}}>=n(k) n\left(k^{\prime}\right)-\frac{\delta_{k k^{\prime}}}{\beta \hbar} \frac{\partial n(k)}{\partial \omega_{k}},
$$

which can be directly checked by differentiating $\partial n(k) / \partial \omega_{k}$. Assuming the thermodynamic limit with the standard replacement

$$
\sum_{k} \rightarrow \frac{V}{(2 \pi)^{3}} \int d \mathbf{k}
$$

and using condition (17.23), one finds

$$
<\hat{\mathbf{P}}^{2}>=-\frac{\hbar V}{(2 \pi)^{3} \beta} \int k^{2} \frac{\partial n(k)}{\partial \omega_{k}} d \mathbf{k} .
$$

Hence, the normal density $(17.30)$ becomes

$$
\rho_{n}=-\frac{\hbar}{3 m_{0}(2 \pi)^{3}} \int k^{2} \frac{\partial n(k)}{\partial \omega_{k}} d \mathbf{k} .
$$

With the ideal-gas Hamiltonian (17.34), the momentum distribution is

$$
n_{k}=\frac{1}{\exp \left\{\beta\left(\hbar \omega_{k}-\mu\right)\right\}-1} .
$$

Specifying the spectrum $\hbar \omega_{k}=\hbar^{2} k^{2} / 2 m_{0}$, one finds the density (17.12) of noncondensed atoms,

$$
\tilde{\rho}=\left(\frac{2 m_{0}}{\beta \hbar^{2}}\right)^{3 / 2} \frac{e^{\beta \mu}}{4 \pi^{2}} \int_{0}^{\infty} \frac{x^{1 / 2} d x}{e^{x}-e^{\beta \mu}},
$$


and the density (17.36) of the normal component,

$$
\rho_{n}=\left(\frac{2 m_{0}}{\beta \hbar^{2}}\right)^{3 / 2} \frac{e^{\beta \mu}}{6 \pi^{2}} \int_{0}^{\infty} \frac{x^{3 / 2} e^{x} d x}{\left(e^{x}-e^{\beta \mu}\right)^{2}} .
$$

The integrals here are related through the equality

$$
\int_{0}^{\infty} \frac{x^{3 / 2} e^{x} d x}{\left(e^{x}-e^{\beta \mu}\right)^{2}}=-\lim _{\lambda \rightarrow 1} \frac{\partial}{\partial \lambda} \int_{0}^{\infty} \frac{x^{1 / 2} d x}{e^{\lambda x}-e^{\beta \mu}} .
$$

Below the condensation temperature, one has $\mu=0$, and the integrals simplify to the form

$$
\int_{0}^{\infty} \frac{x^{p-1} d x}{e^{\lambda x}-1}=\frac{1}{\lambda^{p}} \Gamma(p) \zeta(p) .
$$

In this way, one comes to the conclusion that below the condensation temperature

$$
T_{c}=\frac{2 \pi \hbar^{2}}{m_{0} k_{B}}\left[\frac{\rho}{\zeta(3 / 2)}\right]^{2 / 3}
$$

the density of noncondensed atoms (17.12) and the normal density 17.30 ) coincide,

$$
\tilde{\rho}=\rho_{n}=\rho\left(\frac{T}{T_{c}}\right)^{3 / 2} .
$$

Consequently, the condensate density (17.11) coincides with the superfluid density (17.29).

Such a coincidence is to be treated rather as an occasion than as a rule, since the general forms of the densities (17.12) and (17.30) are very different. The coincidence in Eq. (17.38) happened because of the particular case of an ideal gas with a parabolic spectrum. If, with the same momentum distribution (17.37), the spectrum $\omega_{k}$ is slightly changed, the coincidence of $\tilde{\rho}$ and $\rho_{n}$ will not occur. As an illustration, one may take the phonon spectrum $\omega_{k}=c k$. Then the density of noncondensed particles becomes

$$
\tilde{\rho}=\frac{\zeta(3)}{\pi^{2}}\left(\frac{k_{B} T}{\hbar c}\right)^{3} .
$$

The calculation of the normal density reduces to the integral

$$
\int_{0}^{\infty} \frac{x^{2 n} e^{x} d x}{\left(e^{x}-1\right)^{2}}=2^{2 n-1} \pi^{2 n}\left|B_{2 n}\right|
$$

where $B_{n}$ are the Bernoulli numbers. Equation (17.36) results in

$$
\rho_{n}=\frac{2 \pi^{2}\left(k_{B} T\right)^{4}}{45 m_{0} \hbar^{3} c^{5}}
$$

As is seen, expressions (17.39) and (17.40) are neither coinciding with nor proportional to each other, but they even have different temperature dependence.

For a nonideal system, both the particle spectrum $\omega_{k}$ and momentum distribution $n(k)$ differ from those of the ideal gas, as a result of which the condensate density $\rho_{0}$ is, in general, very different from the superfluid density $\rho_{s}$. The momentum distribution for liquid helium has little in common with that for an ideal gas. Instead of $n(k)$, one often considers the combination $k^{2} n(k)$. The latter, for an ideal gas below $T_{c}$, has the maximum $2 m_{0} k_{B} T / \hbar^{2}$ at $k=0$. But for liquid helium, the function $k^{2} n(k)$ is zero at $k=0$ and possesses a maximum at $k \approx 0.7 \AA^{-1}$, as follows from theoretical calculations 443]- 436, 446, 447] and experiments [454]- 4458]. In this way, there is no general relation between the condensate and superfluid densities. The coincidence of these for the ideal gas with a parabolic spectrum is rather occasional. Moreover, this coincidence is even confusing, since the Landau criterion of superfluidity

$$
\min _{k} \frac{\varepsilon(k)}{k}>0
$$

cannot be satisfied for a parabolic spectrum. Hence, the ideal gas should not posses the property of superfluidity at all. 


\subsection{Spectrum of Collective Excitations}

The most convenient technique for the theoretical description of collective excitations in quantum liquids is the method of Green functions. The spectrum of collective excitations is defined by the poles of the two-particle Green function or by the poles of the density response function $\chi(k, \omega)$. This is equivalent to saying that the collective spectrum $\varepsilon(k)$ is defined by the zeros of the inverted response function $\chi^{-1}(k, \omega)$, that is by the equation

$$
\chi^{-1}(k, \varepsilon(k))=0 .
$$

This method of describing collective excitations can also be employed for nonuniform systems, such as gases of trapped atoms. Therefore, it is worth mentioning here some relevant points of this approach to Bose systems.

The calculation of the density response function for strongly interacting Bose liquids, such as helium, is a very nontrivial task [459]. Actually, there exist no reliable theoretical methods of treating strongly interacting quantum liquids, being based on microscopic theories. Because of this, the consideration here will be limited by weakly nonideal systems, for which the so-called random-phase approximation is valid. This approximation corresponds to the usage of the Hartree form for self-energy. To be more accurate, one has to employ the correlated Hartree approximation 291, 355 taking account of interatomic correlations, as a result of which the bare interaction potential is replaced by the smoothed potential (17.8). This is especially important for atoms interacting through nonintegrable potentials for which the Hartree self-energy diverges because of the divergence of the Fourier transform of the interaction potential, while the Fourier transform

$$
\tilde{\Phi}(k)=\int \bar{\Phi}(r) e^{-i \mathbf{k} \cdot \mathbf{r}} d \mathbf{r}
$$

of the smoothed potential $(\overline{17.8})$ perfectly exists.

Considering collective excitations for the same system, it is very instructive to compare the spectrum obtained under different assumptions, in order to understand what would be the difference between the collective spectra for the cases: (i) when the system is in a coherent state and when it is incoherent, and (ii) when gauge symmetry is broken and when it is conserved. As follows from Chapter 13, collective excitations for a weakly nonideal Bose system in a coherent state possess the same spectrum as that for a system with broken symmetry [282, 300, 445.

For an incoherent system, with conserved gauge symmetry, the single-particle spectrum in the correlated Hartree approximation is

$$
\omega(k)=\frac{k^{2}}{2 m_{0}}+\rho \tilde{\Phi}(0)-\mu .
$$

Here and in what follows, the system of units is used where $\hbar \equiv 1$. Recall that the single-particle spectrum is given by the poles of the single-particle Green function. These poles, when gauge symmetry is conserved, are different from those of the two-particle Green function, giving the spectrum of collective excitations. This is contrary to the case of broken gauge symmetry when the single-particle and collective spectra coincide [282, 445]. The single-particle Green function for a Bose system with conserved gauge symmetry has the form

$$
G(k, \omega)=\frac{1+n(k)}{\omega-\omega(k)+i 0}-\frac{n(k)}{\omega-\omega(k)-i 0},
$$

in which the single-particle spectrum $\omega(k)$ is defined in Eq. (17.43), for the approximation considered, and

$$
n(k)=[\exp \{\beta \omega(k)\}-1]^{-1}
$$

is the momentum distribution. In this case, the density response function becomes

$$
\chi(k, \omega)=\frac{\Pi(k, \omega)}{1-\Pi(k, \omega) \tilde{\Phi}(k)},
$$

with the polarization function

$$
\Pi(k, \omega)=\frac{k^{2}}{(2 \pi)^{3} m_{0}} \int \frac{n\left(k^{\prime}\right) d \mathbf{k}^{\prime}}{\left(\omega-\mathbf{k} \cdot \mathbf{k}^{\prime} / m_{0}\right)^{2}-\left(k^{2} / 2 m_{0}\right)^{2}} .
$$


The equation (17.41) for the spectrum of collective excitations can be written as

$$
1-\Pi(k, \varepsilon(k)) \tilde{\Phi}(k)=0 .
$$

The polarization function (17.45) can be simplified noticing that the momentum distribution $n(k)$ quickly diminishes as $k$ increases. Then one can put $\mathbf{k}^{\prime}=0$ in the denominator of Eq. (17.45), which yields

$$
\Pi(k, \omega)=\frac{\rho k^{2} / m_{0}}{\omega^{2}-\left(k^{2} / 2 m_{0}\right)^{2}} .
$$

Substituting this in Eq. (17.46) results in the Bogolubov spectrum

$$
\varepsilon_{B}(k)=\sqrt{c^{2}(k) k^{2}+\left(\frac{k^{2}}{2 m_{0}}\right)^{2}},
$$

in which

$$
c(k) \equiv \sqrt{\frac{\rho}{m_{0}} \tilde{\Phi}(k)} .
$$

If one assumes here the delta-potential, as in Eq. (9.3), one gets the same Bogolubov spectrum (13.8) as for a coherent system. The same collective spectrum (17.48) follows for a system with broken gauge symmetry [282, 300, 445].

The approach, based on density response functions, can also be applied to a mixture of Bose liquids defined by the Hamiltonian (14.1). It is straightforward to demonstrate [395] that the spectrum of collective excitations branches, and, for a mixture with conserved gauge symmetry, acquires the same form (14.27) as for a coherent mixture of section 14.2 or for a mixture with broken gauge symmetry [393]. The condition of dynamical stability for a binary mixture is

$$
\tilde{\Phi}_{11}(0) \tilde{\Phi}_{22}(0)>\tilde{\Phi}_{12}^{2}(0) \text {. }
$$

For the case of the delta-potentials (14.11), one has to replace $\tilde{\Phi}_{i j}(k)$ by $A_{i j}$, which reduces the inequality (17.50) to condition (14.34).

Moreover, considering the mixture with relative motion of components, it is possible to show that the spectra of collective excitations and, respectively, the conditions of dynamic stability are the same for a coherent mixture discussed in section 14.4, for a mixture with broken gauge symmetry [393], as well as for a normal mixture with conserved gauge symmetry [395]. Thus, the spectrum of collective excitations does not depend on whether the system is coherent or normal, whether gauge symmetry is broken or conserved.

For superfluid ${ }^{4} \mathrm{He}$, the first spectrum of collective excitations was proposed by Landau [460, 461] in the course of analyzing thermodynamic properties. Feynman [462] suggested a microscopic basis for Landau's phenomenological dispersion curve, connecting the excitations spectrum with the static structure factor $S(k)$, which resulted in the spectrum

$$
\varepsilon_{F}(k)=\frac{k^{2} / 2 m_{0}}{S(k)} .
$$

Neither Landau nor Feynman mentioned the broken gauge symmetry. Bogolubov [282, 463], deriving the excitation spectrum, introduced gauge symmetry breaking. But, as is demonstrated above, the same Bogolubov spectrum can be derived without breaking gauge symmetry. All of them, Landau, Feynman and Bogolubov, considered the phonon-roton curve of excitations in helium as a unified branch, so that it is impossible to speak strictly of phonons and rotons as of different types of elementary excitations. But it is more correct to speak of the phonon and roton parts of the same unique spectrum. Note that the Bogolubov spectrum (17.48) can reproduce the phonon-roton spectrum of liquid helium for an appropriate interaction potential defining the effective sound velocity (17.49). This spectrum is reproduced under simple conditions on the Fourier transform $\tilde{\Phi}(k)$ :

$$
\tilde{\Phi}(0)>0, \quad \min _{k} \tilde{\Phi}(k)=\tilde{\Phi}\left(k_{r}\right)<0, \quad \lim _{k \rightarrow \infty} \tilde{\Phi}(k)=0,
$$

where $k_{r}$ corresponds to the point of roton minimum. Such conditions are easy to achieve even for rather simple potentials 464. 
The difficulty of calculating the spectrum of collective excitations for the realistic strongly interacting liquids, such as helium, prompted some authors to construct phenomenological or semiphenomenological models. We shall not give here a complete survey of these models but will mention only one of them, which recently provoked a vivid discussion. This is the model advanced by Glyde and Griffin 465]-468]. The basic assumption of this model is that there are in superfluid ${ }^{4} \mathrm{He}$ two principally different branches of excitations: one is the phonon branch due to density excitations at low wave vectors, and another part is the quadratic single-particle branch at higher wave vectors. These two branches exist independently of each other, so that they remain above as well as below the temperature of superfluid transition $T_{\lambda}$. But below $T_{\lambda}$, these branches become coupled via the appeared Bose condensate accompanied by broken gauge symmetry. However, the existence of two separate branches apparently contradicts the unified picture of Landau, Feynman, and Bogolubov. By exact microscopic consideration, Nepomnyashchy [469] showed that model propagators, employed in the discussed model 4465][468, are not consistent with the general structure of Green function equations, while the latter support the unified nature of the phonon-roton spectrum. The temperature dependence of the excitation spectrum was studied experimentally [470, 471]. These experiments demonstrated that there is no indication of a well-defined single-particle branch, becoming the roton mode, that would suddenly appear as one goes below $T_{\lambda}$. Contrary to this, the superfluid transition is marked by a complete softening of the roton mode and its rapid attenuation, when one approaches $T_{\lambda}$ from below. Above $T_{\lambda}$, the roton mode continues to an overdamped diffusive mode of zero frequency. In this way, experiments 4770, 471, as is concluded by their authors, imply "a qualitative disagreement with the interpretation proposed by Glyde and Griffin".

Thus, the phonon-roton spectrum of superfluid helium must be considered as a unified branch. The question remains whether there could exist some remnants of low-frequency quasiparticle excitations in addition to the phonon-roton branch, with the energies below the broad multiphonon component. There have been some theoretical arguments [395, 472]- 475] concerning the possible existence of an additional quasiparticle excitation branch. In a series of papers 476- 440], the authors find experimental indications that an excitation branch, additional to the phonon-roton spectrum, could exist. However, the experiments have not yet been confirmed by other groups. It is worth emphasizing that, even if some additional excitation branch does exist, one has, first of all, to understand its physical origin and, second, no interpretation should contradict the fact that the phonon-roton curve is a unified branch 469-471.

The phonon-roton spectrum of superfluid ${ }^{4} \mathrm{He}$ has been carefully studied in many experiments (see review [481]). Its commonly accepted form, at saturated-vapor pressure and low temperature $T \leq 1.2 \mathrm{~K}$, is presented in Fig. 43. In the long-wave limit, one has the phonon spectrum

$$
\varepsilon(k) \simeq c_{0} k, \quad c_{0}=2.38 \times 10^{4} \mathrm{~cm} / \mathrm{s} .
$$

In the vicinity of the roton minimum, the dispersion curve is

$$
\varepsilon(k) \simeq \Delta_{r}+\frac{\left(k-k_{r}\right)^{2}}{2 m_{r}},
$$

with $\Delta_{r}=8.6 \mathrm{~K}, k_{r}=1.9 \AA^{-1}, m_{r}=0.16 m_{0}$. But let us stress it again that the phonon and roton parts of the spectrum are the pieces of a unified branch.

The phonon-roton spectrum terminates at around $k=3.5 \AA^{-1}$, becoming unstable with respect to the decay of excitations into several other excitations with lower energies 4482.485.

\subsection{Dynamic Structure Factor}

The spectrum of collective excitations can be experimentally measured by means of neutron scattering described by the double differential cross section

$$
\frac{d^{2} \sigma}{d \Omega d \omega}=b_{s}^{2} \frac{k_{f}}{k_{i}} S(k, \omega)
$$

where $b_{s}$ is the scattering length of a neutron on a helium atom, $k_{i}$ and $k_{f}$ are the initial and final wavevectors of the scattering neutron, and $k$ and $\omega$ are the momentum and energy transfer from the neutron to the sample. 
The dynamics of the liquid are contained in the dynamic structure factor

$$
S(k, \omega)=\frac{1}{2 \pi \rho} \int\left[R(\mathbf{r}, t, 0,0)-\rho^{2}\right] e^{-i(\mathbf{k} \cdot \mathbf{r}-\omega t)} d \mathbf{r} d t,
$$

in which

$$
R\left(\mathbf{r}, t, \mathbf{r}^{\prime}, t^{\prime}\right) \equiv<\hat{\rho}(\mathbf{r}, t) \hat{\rho}\left(\mathbf{r}^{\prime}, t^{\prime}\right)>
$$

is the density-density correlation function, with the density operator

$$
\hat{\rho}(\mathbf{r}, t) \equiv \psi^{\dagger}(\mathbf{r}, t) \psi(\mathbf{r}, t) .
$$

Using the Fourier integral

$$
R\left(\mathbf{r}, t, \mathbf{r}^{\prime}, t^{\prime}\right)=\frac{1}{(2 \pi)^{4}} \int R(k, \omega) e^{i \mathbf{k} \cdot\left(\mathbf{r}-\mathbf{r}^{\prime}\right)-i \omega\left(t-t^{\prime}\right)} d \mathbf{k} d \omega
$$

one gets

$$
S(k, \omega)=\frac{1}{2 \pi \rho}\left[R(k, \omega)-(2 \pi)^{4} \rho^{2} \delta(\mathbf{k}) \delta(\omega)\right] .
$$

Employing the properties of Green functions [291, one can find

$$
R(k, \omega)=-\frac{2 \operatorname{Im} \chi(k, \omega)}{1+e^{-\beta \omega}}+(2 \pi)^{4} \rho^{2} \delta(\mathbf{k}) \delta(\omega) .
$$

Then for the dynamic structure factor (17.54) one has

$$
S(k, \omega)=-\frac{\operatorname{Im} \chi(k, \omega)}{\pi \rho\left(1+e^{-\beta \omega}\right)} .
$$

The response function $\chi(k, \omega)$, on the complex $\omega$-plane, possesses the spectral representation

$$
\chi(k, \omega)=\frac{1}{2 \pi} \int_{-\infty}^{+\infty} \frac{\kappa\left(k, \omega^{\prime}\right)}{\omega-\omega^{\prime}} d \omega^{\prime},
$$

in which the spectral function is

$$
\kappa(k, \omega)=i[\chi(k, \omega+i 0)-\chi(k, \omega-i 0)] .
$$

From the properties of Green functions [291] it follows that

$$
\operatorname{Im} \chi(k, \omega)=-\frac{1}{2} \kappa(k, \omega) \operatorname{coth}\left(\frac{\beta \omega}{2}\right) .
$$

Hence, Eq. (17.56) can be written as

$$
S(k, \omega)=\frac{\kappa(k, \omega)}{2 \pi \rho\left(1-e^{-\beta \omega}\right)} .
$$

The latter, with the notation for the Bose function

$$
b(\omega) \equiv\left(e^{\beta \omega}-1\right)^{-1}
$$

takes the form

$$
S(k, \omega)=\frac{1+b(\omega)}{2 \pi \rho} \kappa(k, \omega) .
$$

The dynamic structure factor satisfies the following sum rules

$$
\int_{-\infty}^{+\infty} S(k, \omega) d \omega=S(k)
$$


which defines the static structure factor $S(k)$,

$$
\int_{-\infty}^{+\infty} \omega S(k, \omega) d \omega=E_{k}
$$

which gives the kinetic energy

$$
E_{k} \equiv \frac{k^{2}}{2 m_{0}}
$$

and

$$
\int_{-\infty}^{+\infty} \frac{S(k, \omega)}{\omega} d \omega=-\frac{\operatorname{Re} \chi(k, 0)}{2 \rho}
$$

The relation of the dynamic structure factor to the density-density correlation function and to the density response function means that these are the density fluctuations which contribute to $S(k, \omega)$. In their turn, the density fluctuations define the spectrum of collective excitations, because of which the dynamic structure factor is directly related to the latter. This relation can be clearly illustrated using the random-phase approximation for the density response function (17.44) and the form (17.47) for the polarization function, which yields

$$
\chi(k, \omega)=\frac{2 \rho E_{k}}{\omega^{2}-\varepsilon^{2}(k)},
$$

where $\varepsilon(k)=\varepsilon_{B}(k)$ is the spectrum of collective excitations in the Bogolubov approximation (17.48). With Eq. (17.66), the spectral function (17.58) becomes

$$
\kappa(k, \omega)=\frac{2 \pi \rho E_{k}}{\varepsilon(k)}[\delta(\omega-\varepsilon(k))-\delta(\omega+\varepsilon(k))] .
$$

Then the dynamic structure factor (17.61) is

$$
S(k, \omega)=[1+b(\omega)] \frac{E_{k}}{\varepsilon(k)}[\delta(\omega-\varepsilon(k))-\delta(\omega+\varepsilon(k))] .
$$

For the static structure factor (17.62) one gets

$$
S(k)=\frac{E_{k}}{\varepsilon(k)} \operatorname{coth} \frac{\beta \varepsilon(k)}{2} .
$$

The sum rule (17.63) is identically valid, and from Eq. (17.65) one has

$$
\int_{-\infty}^{+\infty} \frac{S(k, \omega)}{\omega} d \omega=\frac{E_{k}}{\varepsilon^{2}(k)}
$$

in agreement with the form (17.66).

Expression (17.68) shows that the dynamic structure factor has a sharp peak at the frequency $\omega$ coinciding with the spectrum of collective excitations $\varepsilon(k)$. The delta-function shape of this peak is the result of the simplicity of the approximation used. In reality, the observed peaks are, of course, finite and can be fitted to the measured data by means of the Lorentzian or Gaussian forms.

\subsection{Measurement of Condensate Fraction}

The dynamic structure factor, as is shown above, gives information on the spectrum of collective excitations in liquid helium. Hohenberg and Platzman 486] suggested that this factor can also be used for extracting the value of the condensate fraction

$$
n_{0} \equiv \frac{\rho_{0}}{\rho}
$$


For this purpose, one has to invoke deep-inelastic neutron scattering with very high transferred momenta $k$, such that the scattering could be treated as occurring on single atoms and the scattering atoms could be assumed to be in a free particle state. This implies that the recoil energy $k^{2} / 2 m_{0}$ must be much larger than the mean potential energy $E_{p o t}$ of an atom,

$$
k \gg \sqrt{2 m_{0} E_{\text {pot }}} .
$$

For superfluid helium, with $m_{0}=6.64 \times 10^{-24} \mathrm{~g}$, this gives $k \gg 10 \AA^{-1}$, that is one should have $k \approx 100 \AA^{-1}$. Then, for the dynamic structure factor, the impulse approximation is valid yielding

$$
S_{I A}(k, \omega)=\frac{1}{\rho} \int n(p) \delta\left(\omega-E_{k+p}+E_{p}\right) \frac{d \mathbf{p}}{(2 \pi)^{3}},
$$

where $E_{k}$ is defined in Eq. (17.64). Substituting the momentum distribution

$$
n(k)=(2 \pi)^{3} n_{0} \rho \delta(\mathbf{k})+\tilde{n}(k)
$$

in the impulse approximation (17.73), one has

$$
S_{I A}(k, \omega)=S_{0}(k, \omega)+S_{n}(k, \omega),
$$

with the terms

$$
\begin{gathered}
S_{0}(k, \omega)=n_{0} \delta\left(\omega-E_{k}\right), \\
S_{n}(k, \omega)=\frac{1}{\rho} \int \tilde{n}(p) \delta\left(\omega-E_{k+p}+E_{p}\right) \frac{d \mathbf{p}}{(2 \pi)^{3}} .
\end{gathered}
$$

Hence, the existence of a condensate should result in the appearance of a sharp peak in $S_{0}(k, \omega)$ above the broad distribution due to $S_{n}(k, \omega)$.

For the deep-inelastic scattering, it is convenient to use the West [487] scaling variable

$$
Y \equiv \frac{m_{0}}{k}\left(\omega-E_{k}\right)
$$

and to define the so-called Compton profile

$$
J(k, Y) \equiv \frac{k}{m_{0}} S(k, \omega),
$$

whose name comes from the initial usage of such variables in electron scattering. The convenience of using the profile (17.77) is due to the fact that at high momenta it tends to a value

$$
J(Y) \simeq J(k, Y) \quad(k \rightarrow \infty),
$$

which does not depend on $k$. Thus, for the impulse approximation (17.73), one gets

$$
J_{I A}(Y) \equiv \frac{k}{m_{0}} S_{I A}(k, \omega)=\frac{1}{(2 \pi)^{2} \rho} \int_{|Y|}^{\infty} p n(p) d p .
$$

Inverting the last equation gives the momentum distribution

$$
n(k)=-(2 \pi)^{2} \frac{\rho}{k} \frac{\partial}{\partial k} J_{I A}(k) .
$$

If this distribution would have the form (17.74), one could directly measure the condensate fraction $n_{0}$.

However, there exist several principal difficulties prohibiting the extraction of the momentum distribution from the observed scattering. First, any experimental observation is affected by the statistical uncertainty of the measurements. These uncertainties will translate into uncertainties in the inferred $n(k)$. The most striking feature of the inferred momentum distribution is the increase in the statistical noise near $k=0$, due to the 
division by $k$ in Eq. (17.80). Even very large differences in $n(k)$ at small $k$ only cause small changes in the Compton profile $J(Y)$. Thus, the statistical noise present in $J(Y)$ allows a whole family of $n(k)$ that are consistent with the observed data 458. The predicted small $k$ singular behavior makes little contribution to the observed scattering, and with the experimental techniques now available, will be difficult, if not impossible, to observe. Due to the finite statistical errors inherent in any experiment, the experimental results can not definitely prove the existence of a condensate, which formally corresponds to a $\delta$-function. Some other singular, or even not singular, behavior, but not a condensate, could be responsible for the increase in the scattering at small $k$ observed in the superfluid 458 . Hence, the experimental results can not rule out a ground state which does not contain a condensate or which corresponds to something like a smeared condensate 489. The measured scattering is consistent with many different forms for $n(k)$, including models that do not include a condensate at all 454, 490].

Another weak point in the attempts to measure the condensate fraction in superfluid helium is the usage of the impulse approximation (17.73), which assumes that helium atoms behave as free particles. The latter requires that the transferred momenta satisfy inequality (17.72), being about $100 \AA^{-1}$ for liquid helium. However, the majority of neutron-scattering experiments have been performed at momentum transfers not higher than $23 \AA^{-1}$. Some experiments 491 used the transferred momenta as high as $150 \AA^{-1}$, but the accuracy of these measurements was so low that it did not allow one to decide anything about the value of $n_{0}$.

The fact that helium atoms inside a liquid are not free but strongly interact with their surrounding leads to what one calls the final-state effects [492]-494] and the initial-state effects [495- 497]. The former can be taken into account by defining a convolution

$$
J(Y)=\int_{-\infty}^{+\infty} F\left(Y-Y^{\prime}\right) J_{I A}\left(Y^{\prime}\right) d Y^{\prime}
$$

of the impulse-approximation result with a final-state broadening $F(Y)$ that is to be calculated from a microscopic model 492, 493. Taking account of the initial-state effects requires to change the definition of the scattering variable (17.76) itself 498].

One more problem which is to be taken into account is that what one actually measures is not the profile (17.81), but the effects of instrumental resolution must be involved in order to determine the true scattering. In general, the instrumental broadening is a complicated function depending on the energy and momentum transfer and the instrument geometry, and a simple closed-form expression for the resolution function is not possible. In the case of helium, an effective resolution function $I(Y)$ can be calculated by a Monte Carlo simulation of the spectrometer. In terms of this instrumental resolution function, the observed broadened Compton profile is given by the convolution

$$
J_{o b s}(Y)=\int_{-\infty}^{+\infty} I\left(Y-Y^{\prime}\right) J\left(Y^{\prime}\right) d Y^{\prime}
$$

where $J(Y)$ is defined by Eq. (17.81).

In interpreting experimental scattering data, one usually does the following 449, 499, 500]. Rather than attempt to deconvolute the instrumental resolution and the final-state broadening, one assumes a model profile $J_{\text {mod }}(Y)$, which is substituted in Eq. (17.81) instead of the profile 17.79). After this, one fits the convolutions with $J_{\text {mod }}(Y)$ to the observed scattering profile $J_{o b s}(Y)$. The most often employed model profile [499, 500] is a sum of Gaussians

$$
J_{m o d}(Y)=\frac{A_{1}}{\sqrt{2 \pi} \sigma} \exp \left\{-\frac{\left(Y-Y_{0}\right)^{2}}{2 \sigma_{1}^{2}}\right\}+\frac{A_{2}}{\sqrt{2 \pi} \sigma_{2}} \exp \left\{-\frac{\left(Y-Y_{0}\right)^{2}}{2 \sigma_{2}^{2}}\right\},
$$

whose amplitudes, widths, and common center may be varied. This form is, certainly, not unique, and many other forms could be used to fit the data. These two Gaussians model the two terms in the dynamic structure factor (17.75). The term that is narrower is assumed to model the condensate peak $S_{0}(k, \omega)$, while the wider Gaussian is supposed to model $S_{n}(k, \omega)$. Thus for superfluid helium at $T=0.35 \mathrm{~K}$, one finds [500] $\sigma_{1}=0.95 \AA^{-1}$ and $\sigma_{2}=0.29 \AA^{-1}$, so that the latter width should be related to the condensate. But it is worth noting that even for normal helium the observed scattering is not well characterized by a single Gaussian, and a sum of two Gaussians much better describes the observed scattering. For example, for normal helium at $T=3.5 \mathrm{~K}$, one 
has [500 $\sigma_{1}=1 \AA^{-1}$ and $\sigma_{2}=0.45 \AA^{-1}$. Therefore, the two-Gaussian model may show not the appearance of a condensate but just non-Gaussian behavior of the momentum distribution 499, 500].

In this way, the original goal for much of the work with liquid helium, a direct observation of the condensate fraction, has not come to pass. In view of the current understanding of the final-state effects in helium, it is unlikely that this goal will ever be reached in deep inelastic neutron scattering experiments 449, 458, 500. While the current experimental results do not definitely prove the existence of a condensate, they do provide indirect evidence for its existence, which agrees with many theoretical calculations predicting $n_{0} \approx 10 \%$ at zero temperature.

Several other ways have been suggested for indirectly extracting information on the value of the condensate fraction; the interpretation of such methods being based on model assumptions. Sears 448 tried to determine $n_{0}$ by assuming a relation between the value of the mean kinetic energy

$$
<E_{k}>=\int \frac{k^{2}}{2 m_{0}} n(k) \frac{d \mathbf{k}}{(2 \pi)^{3}}
$$

at $T=T_{\lambda}$ and that value at $T<T_{\lambda}$. The mean kinetic energy could be determined by using the impulse approximation for the dynamic structure factor,

$$
<E_{k}>=\lim _{k \rightarrow \infty} \frac{3 \pi}{4 E_{k}} \int_{-\infty}^{+\infty}\left(\omega-E_{k}\right)^{2} S_{I A}(k, \omega) d \omega .
$$

Campbell [501] suggested to consider a relation between the condensate fraction and the surface tension of superfluid helium. Wyatt 502] studied quantum evaporation from the free surface of liquid ${ }^{4} \mathrm{He}$. The mentioned ways of determining the condensate fraction, being based on several model assumptions, provide the upper limit for $n_{0}$.

An interesting proposal was made by Cummings, Hyland, and Rowlands 503-505 who advanced the relation

$$
\rho^{2}[g(r)-1]=\rho_{n}^{2}\left[g_{n}(r)-1\right],
$$

assumed to be valid for $r \geq 4.5 \AA^{-1}$ and connecting the pair correlation function

$$
g\left(\left|\mathbf{r}-\mathbf{r}^{\prime}\right|\right) \equiv \frac{1}{\rho^{2}}<\psi^{\dagger}(\mathbf{r}) \psi^{\dagger}\left(\mathbf{r}^{\prime}\right) \psi\left(\mathbf{r}^{\prime}\right) \psi(\mathbf{r})>,
$$

measured at $T<T_{\lambda}$, with the pair correlation function $g_{n}(r)$ identified as the function either just above $T_{\lambda}$ or that function extrapolated to the temperature under consideration. The pair correlation function (17.86) and the density-density correlation function (17.53) are connected as

$$
R\left(\mathbf{r}, t, \mathbf{r}^{\prime}, t^{\prime}\right)=\rho^{2} g\left(\left|\mathbf{r}-\mathbf{r}^{\prime}\right|\right)+\rho \delta\left(\mathbf{r}-\mathbf{r}^{\prime}\right) .
$$

From Eqs. (17.54) and (17.62), it follows that

$$
g(r)=1+\frac{1}{(2 \pi)^{3} \rho} \int[S(k)-1] e^{i \mathbf{k} \cdot \mathbf{r}} d \mathbf{k} .
$$

Therefore, the pair correlation function can be calculated by using Eq. (17.88) with the measured static structure factor $S(k)$. Then, by substituting $\rho_{n}=\rho-\rho_{0}$ into the relation (17.85), one has

$$
n_{0}=1-\left[\frac{g(r)-1}{g_{n}(r)-1}\right]^{1 / 2} .
$$

This method of calculating the condensate fraction was employed together with the data for the pair correlation function obtained through neutron scattering [506, 507] and $x$-ray scattering techniques [508, 509]. The values of $n_{0}$, found by applying Eq. (17.89), are in good agreement with those obtained by other methods. However, the derivation of the relation (17.85) was criticized by several authors [313], [510]- 512. The main argument 
against this relation is that the latter does not appropriately take into account the anomalous averages existing in a system with broken gauge symmetry. But if gauge symmetry is conserved, the relation (17.85) can be approximately valid 279 in the region $4 \AA^{-1}<r<12 \AA^{-1}$.

An accurate analysis of different experimental methods of measuring $n_{0}$ was done by Wirth and Hallock [509. They fitted each of the sets of experimental data to the function

$$
n_{0}(T)=n_{0}(0)\left[1-\left(\frac{T}{T_{\lambda}}\right)^{\alpha}\right] .
$$

While there is little theoretical justification for the use of this form for liquid helium, it provides a uniform methodology for obtaining values $n_{0}(0)$. Summarizing the results of various experiments, one has $n_{0}(0) \approx 0.10$ and $5 \leq \alpha \leq 10$.

\section{Concluding Remarks}

Bose-Einstein condensation of trapped atoms is now a very vast and quickly developing branch of physics. Because it is so vast, it is impossible to touch, on a reasonable level of explanation, all related directions in one review. This especially concerns theoretical aspects. Therefore, we preferred to concentrate on the principal points which the theory of nonuniform Bose systems is based on. We have tried to clearly elucidate these main points. The choice of the most important problems is, of course, subjective, and many interesting questions concerning Bose atoms were left aside. The theoretical description of the degenerate trapped Fermi atoms [513, 514 has not been touched at all, as well as the description of trapped Bose-Fermi mixtures [515].

The majority of theoretical considerations here have been based on the Gross-Pitaevskii equation. Temperature effects were only slightly touched. This is because of the following reasons. First of all, it was necessary to concentrate on the principal features of Bose-Einstein condensate at zero or low temperatures, and a detailed discussion of its thermal properties would essentially enlarge the review. Another reason is that there are not yet enough reliable experiments on trapped atoms with Bose-Einstein condensates at finite temperatures which theory could be compared with. Thermal properties of trapped atoms are to be studied more accurately, both theoretically as well as experimentally.

\section{Acknowledgment}

We are very grateful to our colleagues for the kind permission to use figures from their papers. Several theoretical results included in this review have been obtained in collaboration with E.P. Yukalova whose help is very much appreciated. We acknowledge financial support from the São Paulo State Research Foundation FAPESP. Ph.W. C. wishes to thank the Deutscher Akademischer Austauschdienst DAAD for financial support. 


\section{Bibliography}

[1] Bose, S.N., 1924, Z. Phys., 26, 178.

[2] Einstein, A., 1924, Sitz. Ber. Kgl. Preuss. Akad. Wiss., 22, 261.

[3] Anderson, M.H., Ensher, J.R., Matthews, M.R., et al., 1995, Science, 269, 198.

[4] Davis, K.B., Mewes, M.-O., Andrews, M.R., et al., 1995, Phys. Rev. Lett., 75, 3969.

[5] Bradley, C.C., Sackett, C.A., Tolett, J.J., and Hulet, R.G., 1995, Phys. Rev. Lett., 75, 1687.

[6] Han, D.J., Courteille, Ph.W., Wynar, R.H., et al., 1998, Phys. Rev. A, 57, R4114.

[7] Myatt, C.J., Burt, E.A., Ghrist, R.W., et al., 1997, Phys. Rev. Lett., 78, 586.

[8] Stenger, J., Inouye, S., Stamper-Kurn, D.M., et al., 1998, Nature, 396, 345.

[9] Matthews, M.R., Anderson, B.P., Haljan, P.C., et al., 1999, Phys. Rev. Lett., 83, 2498.

[10] Madison, K.M., Chevy, F., Wohlleben, W., and Dalibard, J., 1999, Phys. Rev. Lett. 84, 806.

[11] Inouye, S., Andrews, M.R., Stenger, J., et al., 1998, Nature, 392, 151.

[12] Courteille, Ph.W., Freeland, R.S., Heinzen, D.J., et al., 1998, Phys. Rev. Lett., 81, 69.

[13] Vuletic, V., Kerman, A.J., Chin, Ch., Chu S., 1999, Phys. Rev. Lett., 82, 1406.

[14] Mewes, M.-O., Andrews, M.R., Kurn, D.M., et al., 1997, Phys. Rev. Lett., 78, 582.

[15] Anderson, B.P. and Kasevich, M.A., 1998, Science, 282, 1686.

[16] Bloch, I., Hänsch, T.W., and Esslinger, T., 1999, Phys. Rev. Lett., 82, 3008.

[17] Hagley, E.W., Deng, L., Kozuma, M., et al., 1999, Science, 283, 1706.

[18] Martin, J.L., McKenzie, C.R., Thomas, N.R., et al., 1999, J. Phys. B, 32, 3065.

[19] Hall, D.S., Matthews, M.R., Wieman, C.E., and Cornell, E.A., 1998, Phys. Rev. Lett., 81, 1543.

[20] Kozuma, M., Deng, L., Hagley, E.W., et al., 1999, Phys. Rev. Lett., 82, 871.

[21] Deng, L., Hagley, E.W., Wen, J., et al., 1999, Nature, 398, 218.

[22] Inouye, S., Chikkatur, A.P., Stamper-Kurn, D.M., et al., 1999, Science, 285, 571.

[23] Kozuma, M., Suzuki, Y., Torii, Y., et al., 1999, Science, 287, 2309.

[24] Inouye, S., Pfau, T., Gupta S., et al., 1999, Nature, 402, 641.

[25] Lenz, G., Meystre, P., and Wright, E.M., 1993, Phys. Rev. Lett., 71, 3271. 
[26] Timmermans, E., Tommasini, P., Hussein, M., and Kerman, A., 1999, Phys. Rep., 315, 199.

[27] Ruostekoski, J., Collett, M.J., Graham, R., and Walls, D.F., 1998, Phys. Rev. A, 57, 511.

[28] Goldstein, E.V., Moore, M.G., and Meystre, P., 2000, Laser Phys., 10, 8.

[29] Heurich, J., Moore, M.G., and Meystre, P., 2000, Opt. Commun., 179, 549.

[30] Parkins, A.S. and Walls, D.F., 1998, Phys. Rep., 303, 1.

[31] Dalfovo, F., Giorgini, S., Pitaevski, L.P., and Stringari, S., 1999, Rev. Mod. Phys., 71, 463.

[32] Huang, K., 1987, Statistical Mechanics 2nd ed., (New York: John Wiley and Sons).

[33] Kittel, C., 1976, Elementary Statistical Physics, (New York: John Wiley and Sons).

[34] de Groot, S.R., Hooyman, G.J., and Seldam, C.A.T., 1950, Proc. Roy. Soc. Lond. A, 203, 266.

[35] Bagnato, V.S., Pritchard, D.E., and Kleppner, D., 1987, Phys. Rev. A, 35, 4354.

[36] Yan, Z., 1999, Phys. Rev. A, 59, 4657.

[37] Bagnato, V.S. and Kleppner, D., 1991, Phys. Rev. A, 44, 7439.

[38] Bagnato, V.S., 1996, Phys. Rev. A, 54, 1726.

[39] Grossmann, S. and Holthaus, M., 1995, Phys. Lett. A, 208, 188.

[40] Herzog, C. and Olshanii, M., 1997, Phys. Rev. A, 55, 3254.

[41] Kirsten, K. and Toms, D.J., 1996, Phys. Rev. A, 54, 4188.

[42] Ketterle, W. and van Druten, N.J., 1996, Phys. Rev. A, 54, 656.

[43] Napolitano, R., Luca, J.D., Bagnato, V.S., et al., 1997, Phys. Rev. A, 55, R3954.

[44] Baym, G. and Pethick, C.J., 1996, Phys. Rev. Lett., 76, 6.

[45] Giorgini, S., Pitaevskii, L.P., and Stringari, S., 1997, J. Low Temp. Phys., 109, 309.

[46] Stoof, H.T.C., 1994, Phys. Rev. A, 49, 3824.

[47] Ruprecht, P.A., Holland, M.J., Burnett, K., and Edwards, M., 1995, Phys. Rev. A, 51, 4704.

[48] Fisher, M.E., 1964, J. Math. Phys., 5, 944.

[49] London, F., 1938, Phys. Rev., 54, 947.

[50] Landau, L.D., 1937, Statistical Physics, (Oxford: Butterworth-Heinemann).

[51] Schafroth, M.R., 1954, Phys. Rev., 96, 1442.

[52] Bardeen, J., Cooper, L.N., and Schrieffer, J.R., 1957, Phys. Rev., 106, L162.

[53] Blatt, M., Boër, K., and Brandt, W., 1962, Phys. Rev., 126, 1691.

[54] Chase, L.L., Peyghambarian, N., Grynberg, G., and Mysyrowicz, A., 1979, Phys. Rev. Lett., 42, 1231.

[55] Lin, J.L. and Wolfe, J.P., 1993, Phys. Rev. Lett., 71, 1222.

[56] Hecht, C.E., 1959, Physica, 25, 1159.

[57] Stwalley, W.C. and Nosanow, L.H., 1976, Phys. Rev. Lett., 36, 910. 
[58] Gordon, J.P. and Ashkin, A., 1980, Phys. Rev. A, 21, 1606.

[59] Dalibard, J. and Cohen-Tannoudji, C., 1985, J. Opt. Soc. Am. B, 2, 1070.

[60] Chu, S., Bjorkholm, J.E., Ashkin, A., and Cable, A., 1986, Phys. Rev. Lett., 57, 314.

[61] Frisch, O.R., 1933, Z. Phys., 86, 42.

[62] Kastler, A., 1950, J. Physique, 11, 255.

[63] Hänsch, T.W. and Schawlow, A.L., 1975, Opt. Comm., 13, 68.

[64] Phillips, W.D. and Metcalf, H., 1982, Phys. Rev. Lett., 48, 596.

[65] Ertmer, W., Blatt, R., Hall, J.L., and Zhu, M., 1984, Phys. Rev. Lett., 54, 996.

[66] Stenholm, S., 1986, Rev. Mod. Phys., 58, 699.

[67] Chu, S., Hollberg, L., Bjorkholm, J.E., et al., 1985, Phys. Rev. Lett., 55, 48.

[68] Dalibard, J. and Cohen-Tannoudji, C., 1989, J. Opt. Soc. Am. B, 6, 2023.

[69] Ungar, P., Weiss, D., Riis, E., and Chu, S., 1989, J. Opt. Soc. Am. B, 6, 2058.

[70] Raab, E.L., Prentiss, M., Cable, A., et al., 1987, Phys. Rev. Lett., 59, 2631.

[71] Sesko, D.W., Walker, T.G., and Wieman, C.E., 1991, J. Opt. Soc. Am. B, 8, 946.

[72] Ketterle, W., Davis, K.B., Joffe, M.A., et al., 1993, Phys. Rev. Lett., 70, 2253.

[73] Hemmerich, A. and Hänsch, T.W., 1993, Phys. Rev. Lett., 70, 410.

[74] Grynberg, G., Lounis, B., Verkerk, P., et al., 1993, Phys. Rev. Lett., 70, 2249.

[75] Adams, C.S., Lee, H.J., Davidson, N., et al., 1995, Phys. Rev. Lett., 74, 3577.

[76] Davidson, N., Lee, H.J., Adams, C.S., et al., 1995, Phys. Rev. Lett., 74, 1311.

[77] Andrews, M.R., Kurn, D.M., Miesner, H.-J., et al., 1997, Phys. Rev. Lett., 79, 553.

[78] Stamper-Kurn, D.M., Andrews, M.R., Chikkatur, A.P., et al., 1998, Phys. Rev. Lett., 80, 2027.

[79] Lawall, J., Kulin, S., Saubamea, B., et al., 1995, Phys. Rev. Lett., 75, 4194.

[80] Lee, H.J., Adams, C.S., Kasevich, M., and Chu, S., 1996, Phys. Rev. Lett., 76, 2658.

[81] Depue, M.T., Han, D.J., McCormick, C., et al., 1999, Bull. Am. Phys. Soc., 44, 1008.

[82] Söding, J., Guéry-Odelin, D., Desbiolles, P., et al., 1999, Appl. Phys. B, 69, 257.

[83] Lovelace, R.V.E., Mehanian, C., Tommila, T.J., and Lee, D.M., 1985, Nature, 318, 30.

[84] Migdall, A.L., Prodan, J.V., Phillips, W.D., et al., 1985, Phys. Rev. Lett., 54, 2596.

[85] Petrich, W., Anderson, M.H., Ensher, J.R., and Cornell, E.A., 1995, Phys. Rev. Lett., 74, 3352.

[86] Pritchard, D.E., 1983, Phys. Rev. Lett., 51, 1336.

[87] Bergeman, T., Erez, G., and Metcalf, H.J., 1987, Phys. Rev. A, 35, 1535.

[88] Mewes, M.-O., Andrews, M.R., van Druten, N.J., et al., 1996, Phys. Rev. Lett., 77, 416.

[89] Monroe, C.R., Cornell, E.A., Sackett, C.A., et al., 1993, Phys. Rev. Lett., 70, 414. 
[90] Ernst, U., Marte, A., Schreck, F., et al., 1997, Europhys. Lett., 41, 1.

[91] Esslinger, T., Bloch, I., and Haensch, T.W., 1998, Phys. Rev. A, 58, R2664.

[92] Helmerson, K., Martin, A., and Pritchard, D.E., 1992, J. Opt. Soc. Am. B, 9, 1988.

[93] Newbury, N.R., Myatt, C.J., Cornell, E.A., and Wieman, C.E., 1995, Phys. Rev. Lett., 74, 2196.

[94] Hess, H.F., 1986, Phys. Rev. B, 34, 3476.

[95] Masuhara, N., Doyle, J.M., Sandberg, J.C., et al., 1988, Phys. Rev. Lett., 61, 935.

[96] Davis, K.B., Mewes, M.-O., Joffe, M.A., et al., 1995, Phys. Rev. Lett., 74, 5202.

[97] Ketterle, W. and van Druten, N.J., 1996, Adv. Phys., 37, 181.

[98] Wu, H. and Foot, C.J., 1996, J. Phys. B, 29, L321.

[99] Fried, D.G., Killian, T.C., Willmann, L., et al., 1998, Phys. Rev. Lett., 81, 3811.

[100] Pritchard, D.E., Helmerson, K., and Martin, A.G., 1989, Atomic Physics XI, S. Haroche, J. C. Gay, G. Grynberg, eds. (Singapore: World Scientific), p. 179.

[101] Martin, A.G., Helmerson, K., Bagnato, V.S., et al., 1988, Phys. Rev. Lett., 61, 2431.

[102] Doyle, J.M., Friederich, B., Kim, J., and Patterson, D., 1995, Phys. Rev. A, 52, R2515.

[103] DeMarco, B. and Jin, D.S., 1999, Science, 285, 1703.

[104] Hau, L.V., Busch, B.D., Liu, C., 1998, Phys. Rev. A, 58, R54.

[105] Guéry-Odelin, D., Söding, J., Desbiolles, P., and Dalibard, J., 1998, Europhys. Lett., 44, 25.

[106] Kokkelmans, S.J.J.M.F., Verhaar, B.J., and Gibble, K., 1998, Phys. Rev. Lett., 81, 951.

[107] Prevedelli, M., Cataliotti, F.S., Cornell, E.A., et al., 1999, Phys. Rev. A, 59, 1886.

[108] Shlyapnikov, G.V., Walraven, J.T.M., Rahmanov, U.M., and Reynolds, M.W., 1994, Phys. Rev. Lett., $\mathbf{7 3}, 3247$.

[109] Beijerinck, H.C.W., Vredenbregt, E.D.J., Stas, R.J.W., et al., 2000, Phys. Rev. A, 61 (2).

[110] Bradley, C.C., Sackett, C.A., Hulet, R.G., 1997, Phys. Rev. Lett., 78, 985.

[111] Sackett, C.A., Gerton, J.M., Welling, M., and Hulet, R.G., 1999, Phys. Rev. Lett., 82, 876.

[112] Niering, M., Holtzwarth, R., Reichert, J., et al., 2000, Phys. Rev. Lett., 84, 5496.

[113] Young, B.C., Cruz, F.C., Itano, W.M., and Bergquist, J.C., 1999, Phys. Rev. Lett., 83, 3799.

[114] Hijmans, T.W., Kagan, Y., and Shlyapnikov, G.V., 1993, Phys. Rev. B, 48, 12886.

[115] Ketterle, W., Durfee, D.S., and Stamper-Kurn, D.M., 1999, Proc. Int. School Phys. "Enrico Fermi", CXL, 67.

[116] Holland, M.J., Jin, D.S., Chiofalo, M.L., and Cooper, J., 1997, Phys. Rev. Lett., 78, 3801.

[117] Castin, Y. and Dum, R., 1996, Phys. Rev. Lett., 77, 5315.

[118] Loudon, R., 1983, Quantum Theory of Light, (Oxford: University Press).

[119] Andrews, M.R., Mewes, M.-O., van Druten, N.J., et al., 1996, Science, 273, 84. 
[120] Ensher, J.R., Jin, D.S., Matthews, M.R., et al., 1996, Phys. Rev. Lett., 77, 4984.

[121] Burke, J.P.J., Bohn, J.L., Esry, B.D., and Greene, C.H., 1997, Phys. Rev. A, 55, R2511.

[122] Matthews, M.R., Hall, D.S., Jin, D.S., et al., 1998, Phys. Rev. Lett., 81, 243.

[123] Hall, D.S., Matthews, M.R., Ensher, J.R., et al., 1998, Phys. Rev. Lett., 81, 1539.

[124] Hall, D.S., Ensher, J.R., Jin, D.S., et al., 1998, Proc. SPIE, 3270, 98.

[125] Miesner, H.-J., Stamper-Kurn, D.M., Stenger, J., et al., 1999, Phys. Rev. Lett., 82, 2228.

[126] Stamper-Kurn, D.M., Miesner, H.-J., Chikkatur, A.P., et al., 1999, Phys. Rev. Lett., 83, 661.

[127] Stamper-Kurn, D.M. and Ketterle, W., 2000, Proc. of "Les Houches" Summer School, Session, LXXII.

[128] Law, C.K., Pu, H., and Bigelow, N.P., 1998, Phys. Rev. Lett, 83, 5257.

[129] Jin, D.S., Wieman, C.E., and Cornell, E.A., 1996, Phys. Rev. Lett., 77, 420.

[130] Mewes, M.-O., Andrews, M.R., van Druten, N.J., et al., 1996, Phys. Rev. Lett., 77, 988.

[131] Singh, K.G., Rokhsar, D.S., 1996, Phys. Rev. Lett., 77, 1667.

[132] Edwards, M., Ruprecht, P.A., Burnett, K., et al., 1996, Phys. Rev. Lett., 77, 1671.

[133] Stringari, S., 1996, Phys. Rev. Lett., 77, 2360.

[134] Jin, D.S., Matthews, M.R., Ensher, J.R., et al., 1997, Phys. Rev. Lett., 78, 764.

[135] Onofrio, R., Durfee, D.S., Raman, C., et al., 2000, Phys. Rev. Lett., 84, 810.

[136] Stamper-Kurn, D.M., Miesner, H.-J., Inouye, S., et al., 1998, Phys. Rev. Lett., 81, 500.

[137] Stenger, J., Inouye, S., Chikkatur, A.P., et al., 1999, Phys. Rev. Lett., 82, 4569.

[138] Stamper-Kurn, D.M., Chikkatur, A.P., Görlitz, A., et al., 1999, Phys. Rev. Lett., 83, 2876.

[139] Yukalov, V.I., Yukalova, E.P., and Bagnato, V.S., 1997, Phys. Rev. A, 56, 4845.

[140] Williams, J.E., Walser, R., Cooper, J., et al., 1999, Phys. Rev. A, 61, 33612.

[141] Matthews, M.R., Anderson, B.P., Haljan, P.C., et al., 1999, Phys. Rev. Lett., 83, 3358.

[142] Williams, J.E. and Holland, M., 1999, Nature, 401, 568.

[143] Raman, C., Köhl, M., Onofrio, R., et al., 1999, Phys. Rev. Lett., 83, 2502.

[144] Onofrio, R., Raman, C., Vogels, J.M., et al., 2000, Phys. Rev. Lett., 85, 2228.

[145] Chikkatur, A.P., Görlitz, A., Stamper-Kurn, D.M., et al., 2000, Phys. Rev. Lett., 85, 483.

[146] Guéry-Odelin, D. and Stringari, S., 1999, Phys. Rev. Lett., 83, 4452.

[147] Maragò, O.M., Hopkins, S.A., Arlt, J., et al., 1999, Phys. Rev. Lett., 84, 2056.

[148] Madison, K.M., Chevy, F., Wohlleben, W., and Dalibard, J., 1999, Preprint cond-mat/0004037.

[149] Chevy, F., Madison, K., and Dalibard, J., 2000, Phys. Rev. Lett., 85, 2223.

[150] Anderson, B.P., Haljan, P.C., Wieman, C.E., and Cornell, E.A., 2000, Phys. Rev. Lett., 85, 2857.

[151] Rokhsar, D.S., 1997, Phys. Rev. Lett., 79, 2164. 
[152] Dobrek, L., Gajda, M., Lewenstein, M., et al., 1999, Phys. Rev. A, 60, R3381.

[153] Jackson, B., McCann, J.F., and Adams, C.S., 2000, Phys. Rev. A, 61, 13604.

[154] Butts, D.A. and Rokhsar, D.S., 1999, Nature, 397, 327.

[155] Zambelli, F., and Stringari, S., 1998, Phys. Rev. Lett., 81, 1754.

[156] Morgan, S.A., Ballagh, R.J., and Burnett, K., 1997, Phys. Rev. A, 55, 4338.

[157] Reinhardt, W.P. and Clark, C.W., 1997, J. Phys. B, 30, L785.

[158] Jackson, A.D., Kavoulakis, G.M., and Pethick, C.J., 1998, Phys. Rev. A, 58, 2417.

[159] Muryshev, A.E., van Linden van den Heuwell, H.B., and Shlyapnikov, G.V., 1999, Phys. Rev. A, 60, R2665.

[160] Busch, T. and Anglin, J.R., 1999, Phys. Rev. A, 60, R2669.

[161] Backhaus, S., Pereverzev, S., Simmonds, R.W., et al., 1998, Nature, 392, 687.

[162] Dum, R., Cirac, J.I., Lewenstein, M., and Zoller, P., 1998, Phys. Rev. Lett., 80, 2972.

[163] Burger, S., Bongs, K., Dettmer, S., et al., 1999, Phys. Rev. Lett., 83, 5198.

[164] Denschlag, J., Simsarian, J.E., Feder, D.L., et al., 2000, Science, 287, 97.

[165] Courteille, Ph.W., Han, D.J., Wynar, R.H., et al., 1998, Proc. of the SPIE, 3270, 116.

[166] Soroko, A.V., 1997, J. Phys. B, 30, 5621.

[167] Morinaga, M., Yasuda, M., Kishimoto, T., et al., 1996, Phys. Rev. Lett., 77, 802.

[168] Lamb, Jr., W.E., 1995, Appl. Phys. B, 60, 77.

[169] Mlynek, J., Balykn, V., and Meystre, P., 1992, Appl. Phys. B, 54, 319.

[170] Adams, C.S., Sigel, M., and Mlynek, J., 1994, Phys. Rep., 240, 143.

[171] Pillet Ed., P., 1994, J. Physique II, 4, 1877.

[172] Prokhorov, A.M. et al., 1994, Laser Phys., 4, 829.

[173] Foot, C.J., Wu, H., Arimondo, E., and Morigi, G., 1993, J. Physique, 4, 1913.

[174] Ramsey, N., 1949, Phys. Rev., 76, 906.

[175] Blockley, C.A., Walls, D.F., and Risken, H., 1992, Europhys. Lett., 17, 509.

[176] Wineland, D., Monroe, C., Itano, W.M., et al., 1998, J. Res. Natl. Inst. Stand. Technol., 103, 259.

[177] Timp, G., Behringer, R.E., Tennant, D.M., et al., 1992, Phys. Rev. Lett., 69, 1636.

[178] Landragin, A., Labeyrie, G., Kaiser, R., et al., 1996, Opt. Lett., 21, 1591.

[179] Ruprecht, P.A., Holland, M.J., and Burnett, K., 1994, Phys. Rev. A, 49, 4726.

[180] Balykin, V.I., Letokhov, V.S., Ovchinnikov, Y.B., and Sidorov, A.I., 1988, Phys. Rev. Lett., 60, 2137.

[181] Henkel, C., Westbrook, C.I., and Aspect, A., 1996, J. Opt. Soc. Am. B, 13, 233.

[182] Drndic, M., Zabow, G., Lee, C., et al., 1999, Phys. Rev. A, 60, 4012. 
[183] Bongs, K., Burger, S., Birkl, G., et al., 1999, Phys. Rev. Lett., 83, 3577.

[184] Pritchard, D.E., Rubenstein, R.A., Dhirani, A., et al., 1999, Phys. Rev. A, 59, 4641.

[185] Denschlag, J., Cassettari, D., and Schmiedmayer, J., 1999, Phys. Rev. Lett., 82, 2014.

[186] Hinds, E.A., Bosjier, M.G., and Hughes, I.G., 1998, Phys. Rev. Lett., 80, 645.

[187] Renn, M.J., Montgomery, D., Vdovin, O., et al., 1995, Phys. Rev. Lett., 75, 3253.

[188] Ito, H., Nakata, T., Sakaki, K., et al., 1996, Phys. Rev. Lett., 76, 4500.

[189] Schiffer, M., Rauner, M., Kuppens, S., et al., 1998, Appl. Phys. B, 67, 705.

[190] Bongs, K., Burger, S., Dettmer, S., et al., 2000, Preprint cond-mat/0007381.

[191] Bernet, S., Oberthaler, M.K., Abfalterer, R., et al., 1996, Phys. Rev. Lett., 77, 5160.

[192] Doak, R.B., Grisenti, R.E., Rehbein, D.S., et al., 1999, Phys. Rev. Lett., 83, 4229.

[193] Huesmann, R., Balzer, C., Courteille, Ph.W., et al., 1999, Phys. Rev. Lett., 82, 1611.

[194] Weiss, D.S., Young, B.C., and Chu, S., 1994, Appl. Phys. B, 59, 217.

[195] Andrews, M.R., Townsend, C.G., Miesner, H.-J., et al., 1997, Science, 275, 637.

[196] Wiseman, H.M. and Collett, M.J., 1995, Phys. Lett. A, 202, 246.

[197] Bordé, C.J., 1995, Phys. Lett. A, 204, 217.

[198] Spreeuw, R.J.C., Pfau, T., Janicke, U., and Wilkens, M., 1995, Europhys. Lett., 32, 469.

[199] Holland, M., Burnett, K., Gardiner, C., et al., 1996, Phys. Rev. A, 54, R1754.

[200] Guzman, A.M., Moore, M., and Meystre, P., 1996, Phys. Rev. A, 53, 977.

[201] Moy, G.M., Hope, J.J., and Savage, C.M., 1997, Phys. Rev. A, 55, 3631.

[202] Wiseman, H.M., 1997, Phys. Rev. A, 56, 2068.

[203] Kneer, B., Wong, T., Vogel, K., et al., 1998, Phys. Rev. A, 58, 4841.

[204] Hutchinson, D.A.W., 1999, Phys. Rev. Lett., 82, 6.

[205] Miesner, H.-J., Stamper-Kurn, D.M., Andrews, M.R., et al., 1998, Science, 279, 1005.

[206] Wallis, H. and Steck, H., 1998, Europhys. Lett., 41, 477.

[207] Hagley, E.W., Deng, L., Kozuma, M., et al., 1999, Phys. Rev. Lett., 83, 3112.

[208] Bloch, I., Hänsch, Th. W., and Esslinger, T., 2000, Nature, 403, 166.

[209] Simsarian, J.E., Denschlag, J., Edwards, M., et al., 1999, Phys. Rev. Lett., 85, 2040.

[210] Ketterle, W. and Miesner, H.-J., 1997, Phys. Rev. A, 56, 3291.

[211] Burt, E.A., Ghrist, R.W., Myatt, C.J., et al., 1997, Phys. Rev. Lett., 79, 337.

[212] Lee, E.S., Geckeler, C., Heurich, J., et al., 1999, Phys. Rev. A, 60, 4006.

[213] Josephson, B.D., 1962, Phys. Lett., 1, 251.

[214] Courtois, J.Y., Grynberg, G., Lounis, B., and Verkerk, P., 1994, Phys. Rev. Lett., 72, 3017. 
[215] Ovchinnikov, Y.B., Müller, J.H., Doery, M.R., et al., 1999, Phys. Rev. Lett., 83, 284.

[216] Deng, L., Hagley, E.W., Denschlag, J., et al., 1999, Phys. Rev. Lett., 83, 5407.

[217] Karplus, R. and Neumann, M., 1950, Phys. Rev., 83, 776.

[218] Javanainen, J. and Ruostekoski, J., 1995, Phys. Rev. A, 52, 3003.

[219] Graham, R. and Walls, D., 1996, Phys. Rev. Lett., 76, 1774.

[220] Dalfovo, F., Minniti, C., and Pitaevski, L.P., 1997, Phys. Rev. A, 56, 4855.

[221] Hechenblaikner, G., Maragò, O.M., Hodby, E., et al., 2000, Phys. Rev. Lett., 85, 692.

[222] Timmermans, E., Tommasini, P., Côté, R., Hussein, M., and Kerman, A., 1999, Phys. Rev. Lett., 83, 2691.

[223] Javanainen, J. and Mackie, M., 1999, Phys. Rev. A, 59, R3186.

[224] Goldstein, E.V., Plättner, K., and Meystre, P., 1996, J. Res. Natl. Inst. Stand. Technol., 101, 583.

[225] Trippenbach, M., Band, Y.B., and Julienne, P.S., 1998, Opt. Exp., 3, 530.

[226] Siemers, I., Schubert, M., Blatt, R., et al., 1992, Europhys. Lett., 18, 139.

[227] Kasapi, A., Jain, M., Jin, G.Y., and Harris, S.E., 1995, Phys. Rev. Lett., 74, 2447.

[228] Hau, L.V., Harris S.E., Dutton Z., and Behroozi C.H., 1999, Nature, 397, 594.

[229] Inouye, S., Löw, R.F., Gupta, S., et al., 2000, Preprint cond-mat/0006455.

[230] Kash, M.M., Sautenkov, V.A., Zibrov, A.S., et al., 1999, Phys. Rev. Lett., 82, 5229.

[231] Leonhardt, U. and Piwnicki, P., 2000, Phys. Rev. Lett., 84, 822.

[232] Bonifacio, R., DeSalvo, L., Narducci, L.M., and Dangelo, E.J., 1994, Phys. Rev. A, 50, 1716.

[233] Moore, M.G. and Meystre, P., 1999, Phys. Rev. Lett., 83, 5202.

[234] Law, C.K. and Bigelow, N.P., 1998, Phys. Rev. A, 58, 4791.

[235] Moore, M.G. and Meystre, P., 1999, Phys. Rev. A, 59, R1754.

[236] Cirac, J.I., Lewenstein, M., Molmer, K., and Zoller, P., 1998, Phys. Rev. A, 57, 1208.

[237] Gordon, D. and Savage, C.M., 1999, Phys. Rev. A, 59, 4623.

[238] Dalvit, D.A.R., Dziarmaga, and J., Zurek, W.H., 2000, Phys. Rev. A, 62, 13607.

[239] Sackett, S.A., Kielpinski, D., King, B.E., et al., 2000, Nature, 404, 256.

[240] Rauschenbeutel, A., Nogues, G., Osnaghi, S., et al., 2000, Science, 288, 2024.

[241] Jaksch, D., Briegel, J.-J., Cirac, J.I., et al., 1998, Phys. Rev. Lett., 82, 1975.

[242] Fedichev, P.O., Kagan, Y., Shlyapnikov, G.V., and Walraven, J.T.M., 1996, Phys. Rev. Lett., 77, 2913.

[243] Moerdijk, A.J., Verhaar, B.J., and Nagtegaal, T.M., 1996, Phys. Rev. A, 53, 4343.

[244] Tiesinga, E., Moerdijk, A.J., Verhaar, B.J., et al., 1992, Phys. Rev. A, 46, R1167.

[245] Feshbach, H., 1958, Ann. Phys., 5, 357. 
[246] Moerdijk, A.J., Verhaar, B.J., and Axelsson, A., 1995, Phys. Rev. A, 51, 4852.

[247] Vogels, J.M., Tsai, C.C., Freeland, R.S., et al., 1997, Phys. Rev. A, 56, R1067.

[248] Thorsheim, H.R., Weiner, J., and Julienne, P.S., 1987, Phys. Rev. Lett., 58, 2420.

[249] Roberts, J.L., Claussen, N.R., Burke, J.P.Jr., et al., 1998, Phys. Rev. Lett., 81, 5109.

[250] Cornish, S.L., Claussen, N.R., Roberts, J.L., et al., 2000, Phys. Rev. Lett., 85, 1795.

[251] Drummond, P., Kheruntsyan, K.V., and He, H., 1998, Phys. Rev. Lett., 81, 3055.

[252] Hu, B. and Kuang, L.-M., 2000, Preprint cond-mat/0005003.

[253] Holland, M., Park, J., and Walser, R., 2000, Preprint cond-mat/0005062.

[254] Julienne, P.S., Burnett, K., Band, Y.B., and Stwalley, W.C., 1998, Phys. Rev. A, 58, R797.

[255] Band, Y.B. and Julienne, P.S., 1995, Phys. Rev. A, 51, R4317.

[256] Fioretti, A., Comparat, D., Crubellier, A., and Dulieu, O., 1998, Phys. Rev. Lett., 80, 4402.

[257] Mackie, M., Kowalski, R., and Javanainen, J., 1999, Phys. Rev. Lett., 84, 3803.

[258] Courteille, Ph.W., Freeland, R.S., Wynar, R.H., et al., 1998, unpublished.

[259] Wynar, R.H., Freeland, R.S., Han, D.J., et al., 2000, Science, 287, 1016.

[260] Ter Haar, D., 1964, Elements of Statistical Mechanics (New York: Holt).

[261] Michoel, T. and Verbeure, A., 1999, J. Math. Phys., 40, 1268.

[262] Penrose, O., 1951, Phil. Mag., 42, 1373.

[263] Penrose, O. and Onsager, L., 1956, Phys. Rev., 104, 576.

[264] Coleman, A.J. and Yukalov, V.I., 1991, Mod. Phys. Lett. B, 5, 1679.

[265] Coleman, A.J. and Yukalov, V.I., 1992, Nuovo Cimento B, 107, 535.

[266] Coleman, A.J. and Yukalov, V.I., 1993, Nuovo Cimento B, 108, 1377.

[267] Coleman, A.J. and Yukalov, V.I., 1996, Int. J. Mod. Phys. B, 10, 3505.

[268] Valatin, T.G. and Butler, D., 1958, Nuovo Cimento, 10, 37.

[269] Girardeau, M. and Armowitt, R., 1959, Phys. Rev., 113, 755.

[270] Coniglio, A. and Marinaro, M., 1967, Nuovo Cimento B, 48, 249.

[271] Evans, W.A. B. and Imry, Y., 1969, Nuovo Cimento B, 63, 155.

[272] Coniglio, A. and Mancini, F., 1969, Nuovo Cimento B, 63, 227.

[273] Hasting, R. and Halley, T.W., 1975, Phys. Rev. B, 12, 267.

[274] Kondratenko, P.S., 1975, Theor. Math. Phys., 22, 196.

[275] Nepomnyashchy, Y.A. and Pashitsky, E.A., 1990, J. Exp. Theor. Phys., 71, 98.

[276] Pashitsky, E.A., 1999, Low Temp. Phys., 25, 81.

[277] Yukalov, V.I., 1978, Theor. Math. Phys., 37, 1093. 
[278] Yukalov, V.I., 1980, Physica A, 100, 431.

[279] Yukalov, V.I., 1981, Phys. Lett. A , 83, 26.

[280] Yukalov, V.I., 1981, Physica B, 107, 233.

[281] Yang, C.N., 1962, Rev. Mod. Phys., 34, 694.

[282] Bogolubov, N.N., 1967, Lectures on Quantum Statistics (New York: Gordon and Breach), Vol. 1.

[283] Ginzburg, V.L. and Landau, L.D., 1950, J. Exp. Theor. Phys., 20, 1064.

[284] Ziff, R., Uhlenbeck, G., and Kac, M., 1977, Phys. Rep., 32, 169.

[285] Kirsten, K. and Toms, D.J., 1996, J. Res. Natl. Inst. Stand. Technol., 101, 471.

[286] Haugset, T., Haugerud, H., and Andersen, J.O., 1997, Phys. Rev. A, 55, 2922.

[287] Kirsten, K. and Toms, D.J., 1998, Phys. Lett. A, 243, 137.

[288] Yukalov, V.I. and Yukalova, E.P., 1997, Phys. Part. Nucl., 28, 37.

[289] Yukalov, V.I. and Yukalova, E.P., 1997, Physica A, 243, 382.

[290] Takahashi, K., 1998, Phys. Rev. C, 58, 1341.

[291] Yukalov, V.I., 1998, Statistical Green's Functions (Kingston: Queen's University).

[292] Jordan, T.F., 1969, Linear Operators for Quantum Mechanics (New York: Wiley).

[293] Levine, I.N., 1991, Quantum Chemistry (Englewoog Cliffs: Prentice-Hall).

[294] Thouless, D.J., 1961, Quantum Mechanics of Many-Body Systems (New York: Academic).

[295] Slater, J.C., 1963, Quantum Theory of Molecules and Solids (New York: McGrau-Hill), Vol. 1.

[296] Mandel, L. and Wolf, E., 1995, Optical Coherence and Quantum Optics (New York: Cambridge University).

[297] Csörgo, T., 1999, Heavy Ion Phys., 9, 161.

[298] Zimányi, J. and Csörgo, T., 1999, Heavy Ion Phys., 9, 241.

[299] Berezin, F.A., 1966, Method of Second Quantization (New York: Academic).

[300] Bogolubov, N.N., 1970, Lectures on Quantum Statistics (New York: Gordon and Breach), Vol. 2.

[301] Negele, J. W. and Orland, H., 1988, Quantum Many-Particle Systems (Redwood: Addison-Wesley).

[302] Akhiezer, A.I. and Peletminskii, S.V., 1981, Methods of Statistical Physics (Oxford: Pergamon).

[303] Huang, K., Yang, C.M., and Luttinger, J.M., 1957, Phys. Rev., 105, 776.

[304] Huang, K., 1964, in Studies in Statistical Mechanics, eds. De Boer, J. and Uhlenbeck, G.S. (Amsterdam: North-Holland), p. 1.

[305] Baym, G. et al., 1999, Phys. Rev. Lett., 83, 1703.

[306] Gross, E.P., 1957, Phys. Rev., 106, 161.

[307] Ginzburg, V.L. and Pitaevskii, L.P., 1958, J. Exp. Theor. Phys., 7, 858.

[308] Gross, E.P., 1961, Nuovo Cimento, 20, 454. 
[309] Pitaevskii, L.P., 1961, J. Exp. Theor. Phys., 13, 451.

[310] Gross, E.P., 1963, J. Math. Phys., 4, 195.

[311] Popov, V.N., 1987, Functional Integrals and Collective Excitations (Cambridge: Cambridge University).

[312] Isoshima, T. and Machida K., 1997, J. Phys. Soc. Jpn., 66, 3502.

[313] Griffin, A., 1980, Phys. Rev. B, 22, 5193.

[314] Fonda, L., Chirardi, G.C., Weber, T., and Rimini, A., 1966, J. Math. Phys., 7, 1643.

[315] Wong, J., 1967, J. Math. Phys., 8, 2039.

[316] Dattoli, G., Mignani, R., and Torre, A., 1990, J. Phys. A, 23, 5795.

[317] Mostafazadeh, A., 1999, J. Math. Phys., 40, 3311.

[318] Zhidkov, P.E., 1997, Int. J. Mod. Phys. A, 12, 295.

[319] Zhidkov, P.E., 2000, Mat. Sborn., 191, 43.

[320] Lundh, E., Pethick, C.J., and Smith, H., 1997, Phys. Rev. A, 55, 2126.

[321] Brewczyk, M., Clark, C.W., Lewenstein, M., and Rzazewski, K., 1999, J. Phys. B, 32, L 271.

[322] Yukalov, V.I., 1976, Mosc. Univ. Phys. Bull., 31, 10.

[323] Yukalov, V.I., 1976, Theor. Math. Phys., 28, 652.

[324] Yukalov, V.I., 1977, Physica A, 89, 363.

[325] Caswell, W.E., 1979, Ann. Phys., 123, 153.

[326] Seznec, R. and Zinn-Justin, J., 1979, J. Math. Phys., 20, 1398.

[327] Halliday, I.G. and Suranyi, P., 1980, Phys. Rev. D, 21, 1529.

[328] Stevenson, P.M., 1981, Phys. Rev. D, 23, 2916.

[329] Killinbeck, J., 1981, J. Phys. A, 14, 1005.

[330] Okopińska, A., 1987, Phys. Rev. D, 35, 1835.

[331] Bender, C.M. and Rebhan, A., 1990, Phys. Rev. D, 41, 3269.

[332] Stancu, I. and Stevenson, P.M., 1990, Phys. Rev. D., 42, 2710.

[333] Haugerud, H. and Ravndal, F., 1991, Phys. Rev. D, 43, 2736.

[334] Banerjee, N. and Mallik, S., 1991, Phys. Rev. D, 43, 3368.

[335] Gandhi, S.K. and McKane, A.J., 1994, Nucl. Phys. B, 419, 424.

[336] Arvanitis, C., Geniet, F., Kneur, J.L., and Neveu, A., 1997, Phys. Lett. B, 390, 385.

[337] Krein, G. et al., 1998, Eur. Phys. J. A, 1, 45.

[338] Chiku, S. and Hatsuda, T., 1998, Phys. Rev. D, 57, R6.

[339] Chiku, S. and Hatsuda, T., 1998, Phys. Rev. D, 58, 076001.

[340] Kneur, J.L., 1998, Phys. Rev. D, 57, 1. 
[341] Kneur, J.L., 1998, Nucl. Phys. Proc. Suppl. B, 64, 296.

[342] Evans, T.S., Jones, H.F., and Ritz, A., 1998, Nucl. Phys. B, 517, 599.

[343] Hall, R.L., 1983, J. Math. Phys., 24, 324.

[344] Hall, R.L., 1992, Phys. Rev. A, 45, 7682.

[345] Hall, R.L., 1993, J. Math. Phys., 34, 2779.

[346] Okopińska, A., 1993, Ann. Phys., 228, 19.

[347] Feranchuk, I.D., Komarov, L.I., Nichipor, I.V., and Ulyanenko, A.P., 1995, Ann. Phys., 238, 370.

[348] Dineykhan, M., Efimov, G.V., Gandbold, G., and Nedelko, S.N., 1995, Oscillator Representation in Quantum Physics (Berlin: Springer).

[349] Sissakian, A.N. and Solovtsov, I.L., 1999, Phys. Part. Nucl., 30, 1057.

[350] Duncan, A. and Jones, H.F., 1993, Phys. Rev. D, 47, 2560.

[351] Bender, C.M., Duncan, A., and Jones, H.F., 1994, Phys. Rev. D, 49, 4219.

[352] Guida, R., Konishi, K., and Suzuki, H., 1995, Ann. Phys., 241, 152.

[353] Yukalov, V.I., 1989, Int. J. Mod. Phys. B, 3, 1691.

[354] Yukalov, V.I., 1990, Physica A, 167, 833.

[355] Yukalov, V.I., 1990, Phys. Rev. A, 42, 3324.

[356] Yukalov, V.I., 1991, J. Math. Phys., 32, 1235.

[357] Yukalov, V.I., 1992, J. Math. Phys., 33, 3994.

[358] Yukalov, V.I., and Yukalova, E.P., 1993, Int. J. Mod. Phys., 7, 2367.

[359] Yukalov, V.I., and Yukalova, E.P., 1993, Nuovo Cimento B, 108, 1017.

[360] Yukalov, V.I., and Yukalova, E.P., 1994, Physica A, 206, 553.

[361] Yukalov, V.I., and Yukalova, E.P., 1996, Physica A, 225, 336.

[362] Yukalov, V.I., and Yukalova, E.P., 1999, Ann. Phys., 277, 219.

[363] Weiner, J., Bagnato, V.S., Zilio, S.C., and Julienne, P., 1999, Rev. Mod. Phys., 71, 1.

[364] Moerdijk, A.J., Boesten, H.M., and Verhaar, B.J., 1996, Phys. Rev. A, 53, 916.

[365] Edwards, M. et al., 1996, Phys. Rev. A, 53, 1950.

[366] Yukalov, V.I. and Gluzman, S., 1997, Phys. Rev. Lett., 79, 333.

[367] Gluzman, S. and Yukalov, V.I., 1997, Phys. Rev. E, 55, 3983.

[368] Yukalov, V.I. and Gluzman, S., 1997, Phys. Rev. E, 55, 6552.

[369] Yukalov, V.I., Yukalova, E.P., and Gluzman, S., 1998, Phys. Rev. A, 58, 96.

[370] Gluzman, S. and Yukalov, V.I., 1998, Phys. Rev. E, 58, 4197.

[371] Yukalov, V.I. and Gluzman, S., 1998, Phys. Rev. E, 58, 1359. 
[372] Lieb, E.H., 1990, Bull. Am. Math. Soc., 22, 1.

[373] Lundh, E., Pethick, C.J., and Smith, H., 1998, Phys. Rev. A, 58, 4816.

[374] Stringari, S., 1999, Phys. Rev. Lett., 82, 4371.

[375] Castin, Y. and Dum, R., 1999, Eur. Phys. J. D, 7, 399.

[376] Marzlin, K.P., Zhang, W., and Wright, E.M., 1997, Phys. Rev. Lett., 79, 4728.

[377] Petrosyan, K.G. and You, L., 1999, Phys. Rev. A, 59, 639.

[378] Jackson, B., McCann, J.F., and Adams, C.S., 1998, Phys. Rev. Lett., 80, 3903.

[379] Caradoc-Davies, B.M., Ballagh, R.J., and Burnett, K., 1999, Phys. Rev. Lett., 83, 895.

[380] Marzlin, K.P. and Zhang, W., 1998, Phys. Rev. A, 57, 4761.

[381] Feder, D.L., Clark, C.W., and Schneider, B.I., 1999, Phys. Rev. Lett., 82, 4956.

[382] Quist, M., 1999, Phys. Rev. B, 60, 4240.

[383] Birbrair, B.L., Lapina, L.P., and Sadovnikova, V.A., 1976, Phys. At. Nucl., 24, 491.

[384] Negele, J.W., 1982, Rev. Mod. Phys., 54, 913.

[385] Nesterenko, V.O., 1992, Phys. Part. Nucl., 23, 1665.

[386] Brack, M., 1993, Rev. Mod. Phys., 65, 677.

[387] Nesterenko, V.O., Kleining, W., and de Souza Cruz, F.F., 1999, JINR Commun. E4-99-50 (Dubna).

[388] Konotop, V.V. and Vazquez, L., 1994, Nonlinear Random Waves (Singapore: World Scientific).

[389] Perez-Garcia, V.M. et. al., 1997, Phys. Rev. A, 56, 1424.

[390] Öhberg, P., 1999, Phys. Rev. A, 59, 634.

[391] Sinatra, A. and Castin Y., 2000, Eur. Phys. J. D, 8, 319.

[392] Bashkin, E.P. and Vagov, A.V., 1997, Phys. Rev. B, 56, 6207.

[393] Nepomnyashchy, Y.A., 1974, Theor. Math. Phys., 20, 904.

[394] Colson, W.B. and Fetter, A.L., 1978, J. Low Temp. Phys., 33, 231.

[395] Yukalov, V.I., 1980, Acta Phys. Pol. A, 57, 295 (1980).

[396] Feshbach, H., 1992, Theoretical Nuclear Physics (New York: Wiley).

[397] Kivshar, Y.S., Alexander, T.J., and Turitsyn, S.K., 1999, Preprint cond-mat/9907475.

[398] Ostrovskaya, E.A. et al., 2000, Phys. Rev. A, 61, 031601.

[399] Bogolubov, N.N. and Mitropolsky, Y.A., 1961, Asymptotic Methods in the Theory of Nonlinear Oscillations (New York: Gordon and Breach).

[400] Savard, T.A., O'Hara, K.M., and Thomas, J.E., 1997, Phys. Rev. A, 56, 1095.

[401] Yukalov, V.I., Yukalova, E.P., and Bagnato, V.S., 2000, Laser Phys., 10, 26.

[402] Guckenheimer, J. and Holmes, P., 1986, Nonlinear Oscillations, Dynamical Systems, and Bifurcations of Vector Fields (New York: Springer). 
[403] Yukalov, V.I. and Shumovsky, A.S., 1990, Lectures on Phase Transitions (Singapore: World Scientific).

[404] Caradoc-Davies, B.M., Ballagh, R.J., and Blakie, P.B., 2000, Preprint cond-mat/0003063.

[405] Röhrl, A., Naraschewski, M., Schenzle, A., and Wallis, H., 1997, Phys. Rev. Lett., 78, 4143.

[406] Idziaszek, Z. et al., 1999, Phys. Rev. Lett., 82, 4376.

[407] Helmerson, K., Hutchinson, D., Burnett, K., and Phillips, W.D., 1999, Physics World, N 8, 31.

[408] Yukalov, V.I., 1997, Phys. Rev. A, 56, 5004.

[409] Yukalov, V.I., 1993, Laser Phys., 3, 870.

[410] Yukalov, V.I., 1995, Laser Phys., 5, 970.

[411] Yukalov, V.I., 1996, Phys. Rev. B, 53, 9232.

[412] Yukalov, V.I., 1998, Phys. At. Nucl., 61, 1882.

[413] Fong, P., 1962, Elementary Quantum Mechanics (Reading: Addison-Wesley).

[414] Anderson, E.E., 1971, Modern Physics and Quantum Mechanics (Philadelphia: Saunders).

[415] Yukalov, V.I., 1997, Laser Phys., 7, 998.

[416] Yukalov, V.I. and Yukalova, E.P., 1998, Laser Phys., 8, 890.

[417] Yukalov, V.I. and Yukalova, E.P., 1999, Phys. Lett. A, 253, 173.

[418] Yukalov, V.I., 1999, Phys. Rev. A, 60, 721.

[419] Yukalov, V.I. and Yukalova, E.P., 1999, Laser Phys., 9, 531.

[420] Abramowitz, M. and Stegun, I.A., eds., 1972, Handbook of Mathematical Functions (Washington: National Bureau of Standards).

[421] Snoke, D.W., Wolfe, J.P., and Myzyrowicz, A., 1990, Phys. Rev. B, 41, 11171.

[422] Link, B. and Baym, G., 1992, Phys. Rev. Lett., 69, 2959.

[423] Hasuo, M. et al., 1993, Phys. Rev. Lett., 70, 1303.

[424] Chizhov, A.V., Nazmitdinov, R.G., Shumovsky, A.S., and Yukalov, V.I., 1986, Nucl. Phys. A, 449, 660.

[425] Faessler, A., Buchmann, A.J., Krivoruchenko, M.I., and Matremyanov, B.V., 1997, Phys. Lett. B, 391, 255.

[426] Faessler, A., Buchmann, A.J., and Krivoruchenko, M.I., 1998, Phys. Rev. C, 57, 1458.

[427] Yukalov, V.I., 1998, Laser Phys., 8, 1249.

[428] London, F., 1938, Nature, 141, 643.

[429] Tisza, L., 1938, Nature, 141, 913.

[430] Croxton, C.A., 1974, Liquid State Physics (Cambridge: Cambridge University).

[431] Aziz, R.A. et al., 1979, J. Chem. Phys., 70, 4330.

[432] Aziz, R.A., McCourt, F.R., and Wong, C.C., 1987, Mol. Phys., 61, 1487.

[433] Aziz, R.A. et al., 1992, Mol. Phys., 77, 321. 
[434] Manousakis, E., Pandharipande, V., and Usmani, Q., 1985, Phys. Rev. B, 31, 7002.

[435] Manousakis, E. and Pandharipande, V., 1985, Phys. Rev. B, 31, 7029.

[436] Manousakis, E., Pandharipande, V., and Usmani, Q., 1991, Phys. Rev. B, 43, 13587.

[437] Clements, B.E., Krotschek, E., Smith, J.A., and Campbell, C.E., 1993, Phys. Rev. B, 47, 5239.

[438] Pandharipande, V.R. and Schmidt, K.E., 1977, Phys. Rev. A, 15, 2486.

[439] Vakarchuk, I.A., Gonopolsky, O.L., and Yukhnovsky, I.R., 1979, Theor. Math. Phys., 41, 77.

[440] Yukalov, V.I., 1989, Int. J. Theor. Phys., 28, 1237.

[441] Vakarchuk, I.A. and Ykhnovsky, I.R., 1979, Theor. Math. Phys., 40, 100.

[442] Vakarchuk, I.A., 1989, Theor. Math. Phys., 80, 439.

[443] Vakarchuk, I.A., 1990, Theor. Math. Phys., 82, 438.

[444] Reatto, L. and Chester, G.V., 1966, Phys. Lett., 22, 276.

[445] Lifshits, E.M. and Pitaevskii, L.P., 1978, Statistical Physics (Moscow: Nauka).

[446] Lam, C.C. and Fung, P.C., 1988, Nuovo Cimento D, 10, 247.

[447] Lam, C.C. and Fung, P.C., 1988, Nuovo Cimento D, 10, 915.

[448] Sears, V.F., 1983, Phys. Rev. B, 28, 5109.

[449] Sosnick, T.R., Snow, W.M., and Sokol, P.E., 1990, Phys. Rev. B, 41, 11185.

[450] Snow, W.M. and Sokol, P.E., 1990, J. Low Temp. Phys., 80, 197.

[451] Bogoyavlenskii, I.V., Karnatsevich, L.V., Z.A. Kozlov, and A.V. Puchkov, 1990, Phys. Low Temp., 16, 139.

[452] Mahan, G.D., 1981, Many-Particle Physics (New York: Plenum).

[453] Pollock, E.L. and Ceperley, D.M., 1987, Phys. Rev. B, 36, 8343.

[454] Jackson, H.W., 1974, Phys. Rev. A, 10, 278.

[455] Mook, H.A., 1974, Phys. Rev. Lett., 32, 1167.

[456] Rodriguez, L.J., Gersch, H.A., and Mook, H.A., 1974, Phys. Rev. A, 9, 2085.

[457] Mook, H.A., 1983, Phys. Rev. Lett., 51, 1454.

[458] Sokol, P.E., Silver, R.N., and Clark, J.W., 1989, in Momentum Distributions, eds. Silver, R.N. and Sokol, P.E. (New York: Plenum), p. 1.

[459] Sridhar, R., 1987, Phys. Rep., 146, 259.

[460] Landau, L.D., 1941, J. Exp. Theor. Phys., 11, 592.

[461] Landau, L.D., 1947, J. Phys. (Moscow), 11, 91.

[462] Feynman, R.P., 1954, Phys. Rev., 94, 262.

[463] Bogolubov, N.N., 1947, J. Phys. (Moscow), 11, 23.

[464] Brueckner, K.A. and Sawada, K., 1957, Phys. Rev., 106, 1128. 
[465] Glyde, H.R. and Griffin, A., 1990, Phys. Rev. Lett., 65, 1454.

[466] Stirling, W.G. and Glyde, H.R., 1990, Phys. Rev. B, 41, 4224.

[467] Glyde, H.R., 1992, Phys. Rev. B, 45, 7321.

[468] Glyde, H.R., 1992, J. Low Temp. Phys., 87, 407.

[469] Nepomnyashchy, Y.A., 1992, Phys. Rev. B, 46, 6611.

[470] Montfrooij, W. and Svensson, E.C., 1994, Physica B, 194, 521.

[471] Svensson, E.C., Monfrooij, W., and de Schepper, I.M., 1996, Phys. Rev. Lett., 77, 4398.

[472] Griffin, A. and Payne, S.H., 1986, J. Low Temp. Phys., 64, 155.

[473] Yukalov, V.I., 1991, Mod. Phys. Lett., 5, 725.

[474] Yarunin, V.S., 1991, Physica A, 232, 436.

[475] Yarunin, V.S., 1998, Low Temp. Phys., 24, 130.

[476] Blagoveshchenskii, N.M. et al., 1993, JETP Lett., 57, 414.

[477] Blagoveshchenskii, N.M. et al., 1994, Phys. Rev. B, 50, 16550.

[478] Blagoveshchenskii, N.M. et al., 1994, Physica B, 194, 545.

[479] Blagoveshchenskii, N.M. et al., 1997, Low Temp. Phys., 23, 374.

[480] Bogoyavlenskii, I.V., Puchkov, A.V., Skomorokhov, A.N., and Poupko, S.V., 1997, Physica B, $234,324$.

[481] Woods, A.D.B. and Cowley, R.A., 1973, Rep. Prog. Phys., 36, 1135.

[482] Pitaevskii, L.P., 1959, J. Exp. Theor. Phys., 9, 830.

[483] Pitaevskii, L.P., 1970, JETP Lett., 13, 82.

[484] Nepomnyashchy, Y.A., 1972, JETP Lett., 15, 146.

[485] Nepomnyashchy, Y.A. and Nepomnyashchy, A.A., 1974, J. Exp. Theor. Phys., 38, 134.

[486] Hohenberg, P.C. and Platzman, P.M., 1966, Phys. Rev., 152, 198.

[487] West, G.B., 1975, Phys. Rep., 18, 263.

[488] Sokol, P.E., Sosnick, T.R., and Snow, W.M., 1989, in Momentum Distribution, eds. Silver, R.N. and Sokol, P.E. (New York: Plenum), p. 139.

[489] Shenoy, S.R. and Biswas, A.C., 1977, J. Low Temp. Phys., 28, 191.

[490] Sosnick, T.R., Snow, W.M., Sokol, P.E., and Silver, R.N., 1989, Europhys. Lett., 9, 707.

[491] Ikeda, S. and Watanabe, N., 1987, Phys. Lett. A, 121, 34.

[492] Silver, R.N., 1988, Phys. Rev. B, 38, 2283.

[493] Silver, R.N., 1989, Phys. Rev. B, 39, 4022.

[494] Carraro, C. and Koonin, S.E., 1990, Phys. Rev. Lett., 65, 2792.

[495] Mayers, J., 1989, Europhys. Lett., 10, 727. 
[496] Mayers, J., Andreani, A., and Baciocco, G., 1989, Phys. Rev. B, 39, 2022.

[497] Mayers, J., 1990, Phys. Rev. B., 41, 41.

[498] Stringari, S., 1987, Phys. Rev. B, 35, 2038.

[499] Herwig, K.W. et al., 1990, Phys. Rev. B, 41, 103.

[500] Sosnick, T.R., Snow, W.M., Silver, R.N., and Sokol, P.E., 1991, Phys. Rev. B, 43, 216.

[501] Campbell, L.J., 1983, Phys. Rev. B, 27, 1913.

[502] Wyatt, A.F.G., 1998, Nature, 391, 56.

[503] Cummings, F.W., Hyland, G.J., and Rowlands, G., 1970, Phys. Kondens. Mat., 12, 90.

[504] Hyland, G.J., Rowlands, G., and Cummings, F.W., 1970, Phys. Lett. A, 31, 465.

[505] Cummings, F.W., Hyland, G.J., and Rowlands, G., 1981, Phys. Lett. A, 86, 370.

[506] Sears, V.F. and Svensson, E.C., 1979, Phys. Rev. Lett., 43, 2009.

[507] Svensson, E.C., Sears, V.F., and Griffin, A., 1981, Phys. Rev. B, 23, 4493.

[508] Wirth, F.W., Ewen, D.A., and Hallock, R.B., 1983, Phys. Rev. B, 27, 5530.

[509] Wirth, F.W. and Hallock, R.B., 1987, Phys. Rev. B, 35, 34.

[510] Chester, G.V. and Reatto, L., 1980, Phys. Rev. B, 22, 5199.

[511] Fetter, A., 1981, Phys. Rev. B, 23, 2425.

[512] Griffin, A., 1987, Can. J. Phys., 65, 1368.

[513] Bruun, G., Castin, Y., Dum, R., and Burnett, K., 1999, Eur. Phys. J. D, 7, 433.

[514] Bruun, G.M. and Clark, C.W., 1999, Preprint cond-mat/9906392.

[515] Miyakawa, T., Oda, K., Suzuki, T., and Yabu, H., 2000, J. Phys. Soc. Jpn, 69, 2779. 


\section{Figure Captions}

Fig. 1.

Condensed fraction and heat capacity at the phase transition for a homogeneous gas (dotted line) and for a harmonically trapped gas (solid line).

Fig. 2.

Peak density at the phase transition for a harmonically trapped ideal Bose-gas of $10^{6}$ rubidium atoms. The trap secular frequency is set to $\omega_{\text {trap }}=2 \pi 16 \mathrm{~Hz}$.

Fig. 3 .

Heat capacity at the phase transition for $N=100$ (continuous line), $N=1000$ (dashed line) and $N=10000$ (dotted line).

Fig. 4 .

Time-of-flight absorption pictures above (a), slightly below (b), and well below the phase transition (c) (figures taken from $[6]$ ).

Fig. 5 .

Two-photon absorption spectrum of hydrogen. The narrow Doppler-free peak at negative detunings and the broad Doppler-sensitive peak at positive detunings acquire characteristic shoulders when a BEC is present (courtesy of [99]).

Fig. 6.

Measurement of the scaled release energy per particle versus reduced temperature at the phase transition. Straight line is ideal Boltzmann-gas, dashed line finite number ideal Bose-gas [39] and solid curved line fit to the data (courtesy of 120).

Fig. 7.

Temporal evolution of the aspect ratio of suddenly released BECs. The cloverleaf trap had the trapping frequencies $\omega_{r}=2 \pi \cdot 248 \mathrm{~Hz}$ and $\omega_{z}=2 \pi \cdot 16 \mathrm{~Hz}$ (courtesy of 115).

Fig. 8.

Compression oscillations in experiment (dots) and theory (solid line) along the radial and axial directions (courtesy of [122]).

Fig. 9.

Measured (upper curve) and calculated (lower curve) Rabi oscillations of the space-integrated fractional population of the lower hyperfine state (courtesy of 142]).

Fig. 10.

Impurity scattering within a BEC. Elastic collisions between the condensate and impurity atoms traveling at $6 \mathrm{~cm} / \mathrm{s}$ (towards the left in images) distributed the momentum of the collision partners over a sphere showing up as a halo in $50 \mathrm{~ms}$ time-of-flight absorption images (a). In Fig. (b) a Stern-Gerlach type magnetic field gradient has been applied to separate the $m_{F}=0$ atoms from the $m_{F}=-1$ condensate. The fringes are an imaging artifact (courtesy of 145). 
Fig. 11.

Density distribution (a) of the vortex state (the visible atoms are in the upper hyperfine state), (b) after a $\pi / 2$ pulse, and (c) after a $\pi$ pulse (the visible atoms are in the lower hyperfine state). The images (d) and (e) visualize the phase slip around the vortex (courtesy of [9]).

Fig. 12.

Array of 7, 8, and 11 vortices in a Bose-Einstein condensate stirred by a laser beam. The absorption image was taken after a $27 \mathrm{~ms}$ period of free expansion (courtesy of 148 ).

Fig. 13.

Dark solitons in a Bose-Einstein condensate. The images (A to E) show experimental measurements, and the images ( $\mathrm{F}$ to $\mathrm{J}$ ) are calculated density distributions for various times after a phase imprint of $1.5 \pi$ on the top half of the condensate. A positive density disturbance moved rapidly in the $+x$ direction, and a dark soliton moved oppositely and significantly slower than the speed of sound (reprinted with permission from [164).

Fig. 14.

Bosonic stimulation. The curves show the growth of the condensate towards thermal equilibrium after a sudden initial desequilibration for various initial numbers of condensed atoms (courtesy of [205]).

Fig. 15.

Scheme of the setup for interference observation. A cigar-shaped condensate is built in a cloverleaf trap, it is split into two parts with a blue-detuned far-off resonance laser beam, suddenly released from the trap and partially illuminated by a laser light sheet. The interference patterns are recorded by absorption imaging.

Fig. 16.

Interference patterns of two released condensates recorded with the setup sketched in Fig. 15 for three different values of the height of the potential barrier (i.e. intensity of the laser light sheet that separates the trapped condensates). The three pictures on the right hand side are calculated patterns [206] (courtesy of [115]).

Fig. 17.

Output coupling of parts of a BEC by irradiation of radiofrequency pulses (courtesy of [14]).

Fig. 18.

Bragg scattering for matter-waves. The figure on the left shows the geometric arrangement used in the experiments [20, 137, 138]. Short pulses of Raman beams enclosing the angle $\vartheta$ and detuned by $\Delta \omega$ from one another are shone into the BEC. The figure in the right shows the parabolic dispersion relation, which strictly holds only in the limit of negligible mean-field interaction.

Fig. 19.

Bragg spectroscopy of recoil-induced resonances. Fig. (a) shows the shift (solid line) and halfwidth (gray area) of the RIR in the case of particle-like excitations, $\varepsilon_{\text {part }}(p)=h \cdot 100 \mathrm{kHz}$, as a function of density. Fig. (b) shows the RIR shift $\Delta \varepsilon$ and Fig. (c) shows the RIR strength $S(p)$, i.e. the fraction of atoms deflected into the first Bragg order, for phonon-like excitations, $\varepsilon_{p h o n}(p)=h \times 1.54 \mathrm{kHz}$.

Fig. 20.

Four-wave mixing can be illustrated in the laboratory frame (a), in the moving frame defined by $p_{1}=-p_{3}$ (b), and in the moving defined by $p_{1}=-p_{2}$ (c) and accordingly be interpreted in different ways (see text). 
Fig. 21.

False color absorption picture of the atomic density distribution after $4 \mathrm{WM}$ after $6 \mathrm{~ms}$ time of flight. The newly created wavepacket $\psi_{4}$ is smaller than the others (reprinted with permission from [21]).

Fig. 22.

Superradiant Rayleigh scattering (reprinted with permission from [22]). The time-of-flight images show the momentum distribution of the condensate after irradiation of a single laser pulse polarized perpendicularly to the long axis and having the durations (a) $35 \mu \mathrm{s}$, (b) $75 \mu \mathrm{s}$ and (c) $100 \mu \mathrm{s}$. For the longer pulse durations, repeated scattering processes give rise to additional peaks.

Fig. 23.

Feshbach resonance in collisions of ground-state ${ }^{85} \mathrm{Rb}$ atoms 12 . The atoms collide in the $f=2+f=2$ channel (scattering wavefunction $u_{\text {coll }}$ ). A vibrational bound state of the $f=3+f=3$ channel has almost the same energy (wavefunction $u_{r e s}$ ). As the energies are tuned to resonance, the wavefunction $u_{r e s}$ is resonantly enhanced.

Fig. 24.

Magnetic field dependence of the scattering length close to the strong Feshbach resonance near $156 \mathrm{G}$ in ${ }^{85} \mathrm{Rb}$ atoms. The shaded area emphasizes the range of positive values of the scattering length.

Fig. 25.

Free-bound-bound two-photon photoassociation in ${ }^{87} \mathrm{Rb}$. While two ${ }^{2} S_{1 / 2}, f=1, m_{f}=-1$ ground state atoms in the hyperfine state are colliding, they may undergo a photoassociative Raman transition to the bound vibrational state $v=-2, l=0, F=2, m_{F}=-2$ located $636.0094 \mathrm{MHz}$ below the ionization threshold. The intermediate excited state is $v, J=0$ at $12555 \mathrm{~cm}^{-1}$ of the $0_{g}^{-}$potential connected to the ${ }^{2} S_{1 / 2}-{ }^{2} P_{1 / 2}$ asymptote. The levels are chosen to optimize the Franck-Condon overlap.

Fig. 26.

The ground-state energy for the one-dimensional nonlinear Schrödinger equation. The self-similar approximant $E_{*}(g)$ (solid line) is given by Eq. (11.25), the crossover approximant $E_{2}^{*}(g)$ (dashed line) is defined in Eq. (11.21), and the Thomas-Fermi approximant $E_{T F}(g)$ (dashed line with diamonds) is the energy (11.28).

Fig. 27.

The density (11.29) for the corresponding wave functions in the self-similar approximation (11.24) (solid line), Gaussian approximation (11.26) (dashed line), and Thomas-Fermi approximation (11.27) (dashed line with diamonds) for different coupling parameters: (a) $g=0.2$; (b) $g=1$; (c) $g=5$; (d) $g=20$; (e) $g=50$; (f) $g=100$.

Fig. 28.

The residual $R(x)$ defined in Eq. (11.30) for the self-similar solution (11.24) (solid line), Gaussian solution (11.26) (dashed line), and Thomas-Fermi solution (11.27) (dashed line with diamonds) for several coupling parameters: (a) $g=5$; (b) $g=50$; (c) $g=100$.

Fig. 29.

The ground-state energy of atoms confined in a spherically symmetric trap: The self-similar approximant (11.39) (solid line); second-order crossover approximant (11.32) (dashed line); and the Thomas-Fermi energy (9.34) (short-dashed line). 
Fig. 30.

Percentage errors of the first crossover approximants for the ground-state energy of a spherical trap: $E_{1}^{*}(s)$ (solid line); $E_{2}^{*}(s)$ (dashed line); $E_{3}^{*}(s)$ (short-dashed line).

Fig. 31.

Percentage errors of the higher crossover approximants for the ground-state energy of a spherical trap: $E_{2}^{*}(s)$ (solid line); $E_{3}^{*}(s)$ (dashed line); $E_{4}^{*}(s)$ (short-dashed line); $E_{5}^{*}(s)$ (dotted line).

Fig. 32 .

Percentage errors of $E_{1}^{*}$ (solid line), $E_{2}^{*}$ (dashed line) and $E_{3}^{*}$ (short-dashed line) as functions of the coupling $g$ for several energy levels and trap shapes: (a) $\nu=0.1, n=m=k=0$ (ground-state); (b) $\nu=0.1, n=$ $k=0, m=1$ (vortex state); (c) $\nu=0.1, n=3, m=2, k=1 ;$ (d) $\nu=100, n=m=k=0 ;(\mathrm{e})$ $\nu=100, n=k=0, m=2$.

Fig. 33.

The ground-state energy of atoms confined in a cylindrical trap with $\nu=10$ : The optimized approximant (10.54) (solid line) and the Thomas-Fermi energy (9.34) (dashed line).

Fig. 34.

The vortex energies as functions of $\nu g$ : the basic-vortex energy $\Omega_{010}$ (solid line) and the energy $\Omega_{020}$ of the vortex with the winding number $m=2$ (dashed line).

Fig. 35.

The self-similar crossover approximants $f_{k}^{*}(r)$ for the vortex as compared to exact numerical data marked by diamonds: $f_{1}^{*}(r)$ is shown by the solid line; $f_{2}^{*}(r)$, by the long-dashed line; $f_{3}^{*}(r)$, by the short-dashed line; and $f_{4}^{*}(r)$ is presented by the dotted line.

Fig. 36.

The fractional populations $n_{0}(t)$ (dashed line) and $n_{j}(t)$ (solid line) as functions of dimensionless time, measured in units of $\alpha^{-1}$. The transition amplitude is fixed, $b=0.4999$, and the detuning is varied: (a) $\delta=0$; (b) $\delta=0.0001$; (c) $\delta=0.0001001$; (d) $\delta=0.00011$.

Fig. 37.

The time dependence of the fractional populations $n_{0}(t)$ (dashed line) and $n_{j}(t)$ (solid line) under the fixed detuning $\delta=0$ and varied transition amplitude: (a) $b=0.45$; (b) $b=0.4999$; (c) $b=0.5$; (d) $b=0.5001$; (e) $b=0.6 ;$ (f) $b=1$.

Fig. 38.

Excitation of the radial dipole mode with the quantum numbers $n=1, m=0, k=0$ with the parameters $g=100, \nu=10, b=0.4, \delta=0.01$. The ground-state density $\rho_{0}$ (solid line) and the density $\rho_{100}$ (dashed line) as functions of the radial variable $r$ at the point $z=0$ for different times measured in units of $\alpha^{-1}$ : (a) $t=0$; (b) $t=2$; (c) $t=4$.

Fig. 39.

Excitation of the vortex mode with $n=0, m=1, k=0$ with the same parameters as in Fig. 38 . The ground-state density $\rho_{0}$ (solid line) and the density $\rho_{010}$ (dashed line) as functions of the radial variable $r$ at the point $z=0$ for different times: (a) $t=2$; (b) $t=4$. 
Fig. 40.

Excitation of the axial dipole mode, with $n=0, m=0, k=1$ with the same parameters as in Fig. 38 . The ground-state density $\rho_{0}$ (solid line) and the density $\rho_{001}$ (dashed line) as functions of the axial variable $z$ at the point $r=0$ for different times: (a) $t=0$; (b) $t=2$; (c) $t=4$.

Fig. 41.

Phase portrait for the period of time $0 \leq t \leq 50$ for atoms starting from the trap center $x_{0}=y_{0}=z_{0}=0$ with velocities $\dot{x}_{0}, \dot{y}_{0}, \dot{z}_{0}$ varied in the interval [-0.1,0.1]. The trap parameters are $R=10, L=10$, and $\lambda=20$. Note that the picture practically does not change upon independently varying the trap radius and length between 10 and 100. The gravity parameters are $\delta_{x}=0.01, \delta_{y}=0, \delta_{z}=-0.01$. Shown are: (a) trajectories; (b) velocities.

Fig. 42.

Trajectories and velocities during the period of time $0 \leq t \leq 50$ for atoms with the same initial conditions as in Fig. 41, but for the trap parameters $R=1, L=1$, and $\lambda=20$, and for the gravity parameters $\delta_{x}=0.05, \delta_{y}=0, \delta_{z}=-0.05$. Here: (a) trajectories; (b) velocities.

Fig. 43.

Spectrum of collective excitations in superfluid ${ }^{4} \mathrm{He}$ at saturated-vapor pressure and low temperature. The energy $\varepsilon(k)$ is measured in $\mathrm{K}$ and the wave vector $k$ in $\AA^{-1}$. 


\section{Table Captions}

Table 1.

Critical temperature, condensed fraction, heat capacity and its discontinuity at the phase transition for various trapping potentials. $V$ denotes a three-dimensional and $S$ a two-dimensional volume.

Table 2.

Nuclear spin, scattering lengths and transition parameters for various isotopes. The fifth, sixth and seventh column give the linewidth and the transition frequencies of the $D 1$ and $D 2$ lines, where applicable. The last column gives the ground state hyperfine splitting, where applicable.

\section{Table 3.}

Characteristic length scales for elementary excitations. 
Table 1

\begin{tabular}{||l|l|l|l|l||}
\hline \hline$U(\mathbf{r})$ & $\eta$ & $k_{B} T_{c}^{0}$ & $C\left(T_{c}^{-}\right) / N k_{B}$ & $\Delta C\left(T_{c}^{0}\right) / N k_{B}$ \\
\hline \hline $3 \mathrm{D}$ box & $\frac{3}{2}$ & {$\left[\frac{N h^{3}}{(2 \pi m)^{3 / 2}} \frac{1}{V g_{3 / 2}(1)}\right]^{2 / 3}$} & 1.92 & 0 \\
\hline$\left(\frac{z}{a}\right)^{2}$ & 2 & {$\left[\frac{N h^{3}}{(2 \pi m)^{3 / 2}} \frac{1}{\pi^{2} a S g_{2}(1)}\right]^{1 / 2}$} & 4.38 & 0 \\
\hline $\left.\begin{array}{c}\left\{\begin{array}{l}\frac{z}{a}, \quad z>0 \\
\infty,\end{array}, z<0\right.\end{array}\right\}$ & $\frac{5}{2}\left[\frac{N h^{3}}{(2 \pi m)^{3 / 2}} \frac{1}{a S 1.4}\right]^{2 / 5}$ & 6.88 & 3.35 \\
\hline$\left(\frac{x}{a}\right)^{2}+\left(\frac{y}{b}\right)^{2}+\left(\frac{z}{c}\right)^{2}$ & 3 & {$\left[\frac{N h^{3}}{(2 \pi m)^{3 / 2}} \frac{1}{\pi^{3 / 2} a b c g_{3}(1)}\right]^{1 / 3}=\hbar \omega_{\text {trap }}\left(\frac{N}{g_{3}(1)}\right)^{1 / 3}$} & 10.82 & 6.57 \\
\hline \hline
\end{tabular}


Table 2

\begin{tabular}{|c|c|c|c|c|c|c|c|}
\hline Element & $\overline{I I}$ & $\overline{\overline{a_{\text {mixed }}}}$ & $\overline{\overline{a_{\text {triplett }}}}$ & $\overline{\overline{\gamma_{D 2} / 2 \pi}}$ & $\overline{\bar{D} 1}$ & $\overline{\bar{D} 2}$ & 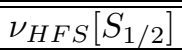 \\
\hline & & {$\left[a_{B}\right]$} & {$\left[a_{B}\right]$} & $\mathrm{MHz}]$ & $\left.\mathrm{cm}^{-1}\right]$ & $\left.\mathrm{cm}^{-1}\right]$ & $\mathrm{MHz}$ \\
\hline${ }^{\overline{1} \mathrm{H}}$ & $\overline{1 / 1 / 2}$ & & $\overline{1.23}$ & \multirow[t]{2}{*}{99.58} & \multirow[t]{2}{*}{82264.} & \multirow[t]{2}{*}{82264.} & \\
\hline${ }^{2} \mathrm{H}$ & 1 & & -6.8 & & & & \\
\hline${ }^{6} \mathrm{Li}$ & 1 & & -2160 & \multirow[t]{2}{*}{5.92} & \multirow[t]{2}{*}{14901.} & \multirow[t]{2}{*}{14901.} & 228.2 \\
\hline${ }^{7} \mathrm{Li}$ & $3 / 2$ & 10 & -27.3 & & & & 803.5 \\
\hline $23 \mathrm{Na}$ & $3 / 2$ & 52 & 85 & 10.01 & 16956. & 16973. & 1771.6 \\
\hline${ }^{39} \mathrm{~K}$ & $3 / 2$ & 118 & 81.1 & \multirow{3}{*}{6.09} & \multirow{3}{*}{12985.} & \multirow{3}{*}{13043.} & 461.7 \\
\hline${ }^{40} \mathrm{~K}$ & 4 & 158 & 1.7 & & & & -1285.8 \\
\hline${ }^{41} \mathrm{~K}$ & $3 / 2$ & 225 & 286 & & & & 254.0 \\
\hline${ }^{85} \mathrm{Rb}$ & $5 / 2$ & -450 & -363 & \multirow[t]{2}{*}{5.98} & \multirow[t]{2}{*}{12579 . } & \multirow[t]{2}{*}{12816.} & 3035.7 \\
\hline${ }^{87} \mathrm{Rb}$ & $3 / 2$ & 105 & 109.3 & & & & 6834.7 \\
\hline${ }^{133} \mathrm{Cs}$ & $7 / 2$ & -240 & -350 & \multirow[t]{2}{*}{5.18} & \multirow[t]{2}{*}{11182.} & \multirow[t]{2}{*}{11737.} & 9192.6 \\
\hline${ }^{135} \mathrm{Cs}$ & $7 / 2$ & 163 & 138 & & & & \\
\hline
\end{tabular}


Table 3

\begin{tabular}{||l|l|l||}
\hline \hline regime & $k^{-1}$ & method \\
\hline \hline hydrodynamic & $\gg l_{m f p}$ & large BECs, high temperatures \\
\hline collisionless & $\ll l_{m f p}$ & trap modulation \\
\hline collective discrete modes & $\gg a_{t r a p}$ & trap modulation, standing soundwave \\
\hline pulsed localized modes & $\ll a_{\text {trap }}$ & dipole force laser beam, propagating soundwave \\
\hline phonon-like & $\gg \xi$ & Bragg scattering \\
\hline free particle-like & $\ll \xi$ & Bragg scattering \\
\hline \hline
\end{tabular}


Figure

Figure

Figure

Figure

Figure

Figure

Figure

Figure

Figure

Figure

Figure

Figure

Figure

Figure

Figure

Figure

Figure

Figure

Figure

Figure

Figure

Figure

Figure

Figure

Figure

Figure

Figure

Figure

Figure

Figure

Figure

Figure

Figure

Figure

Figure 
Figure

Figure

Figure

Figure

Figure

Figure

Figure

Figure

Table

Table

Table 

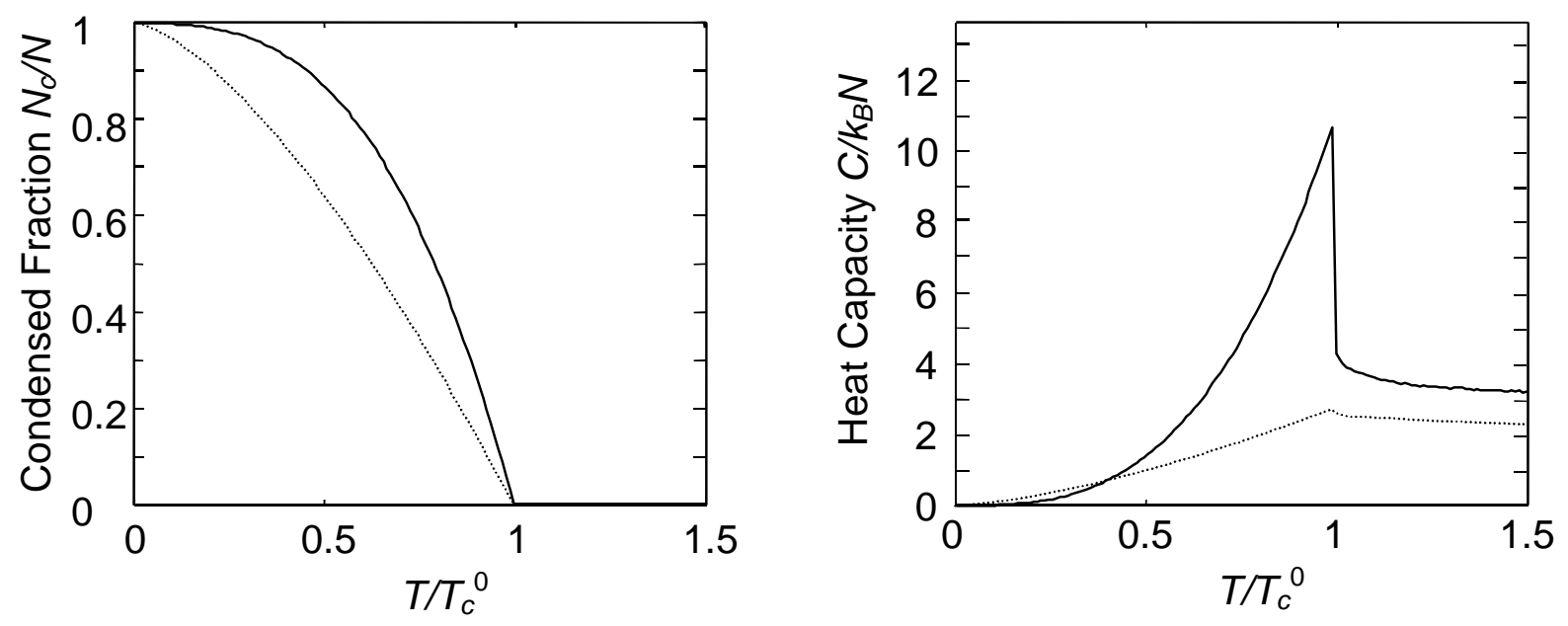


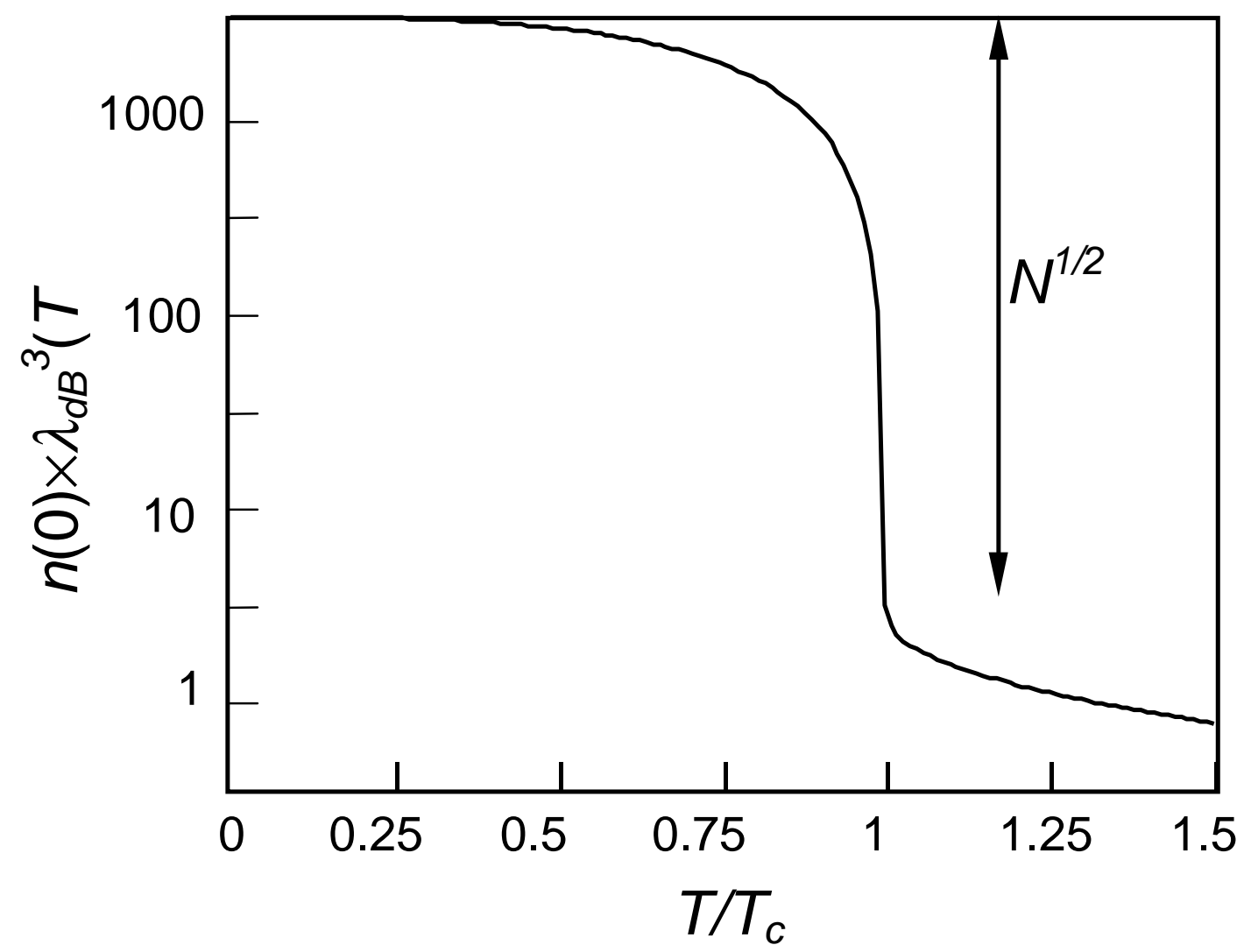




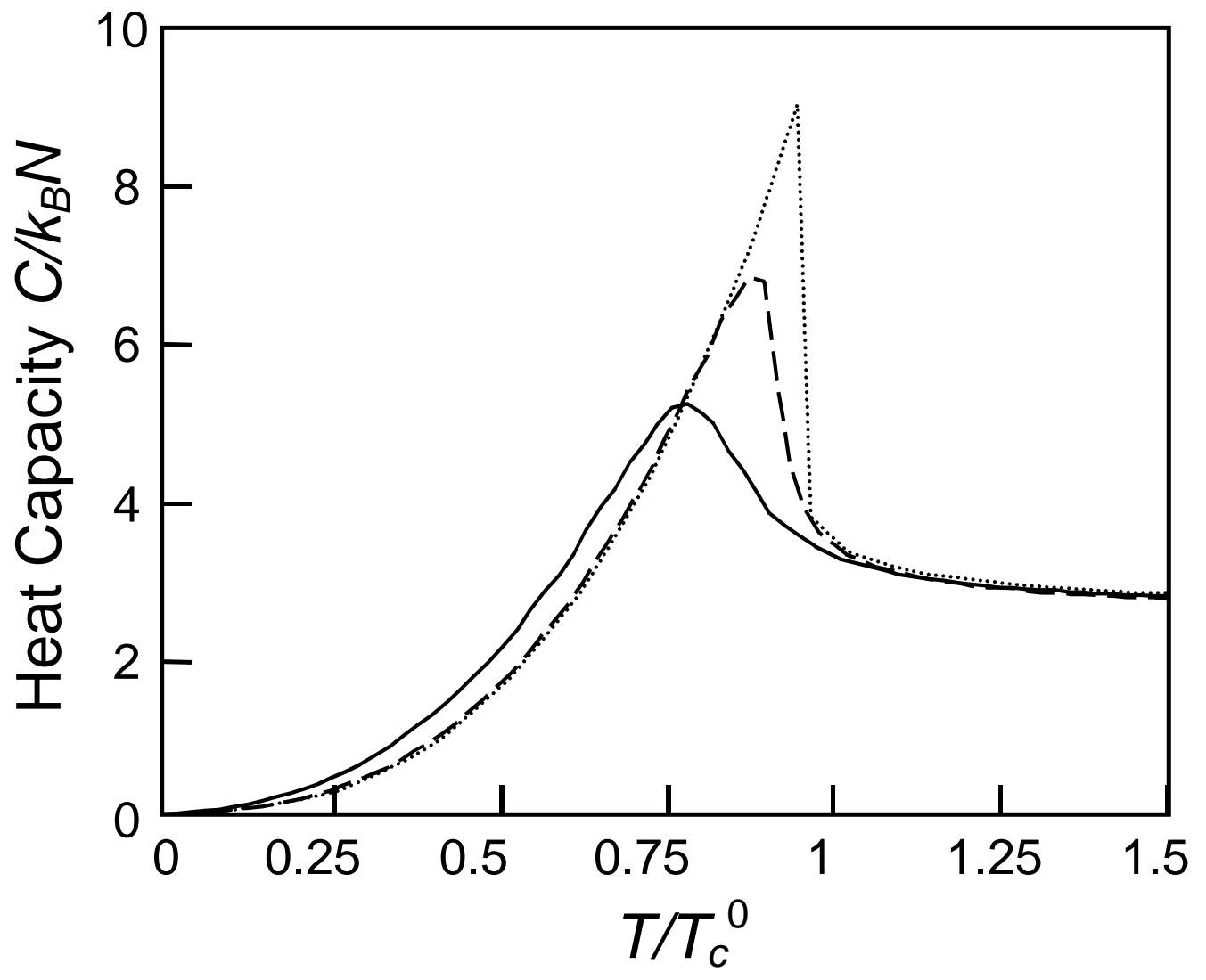




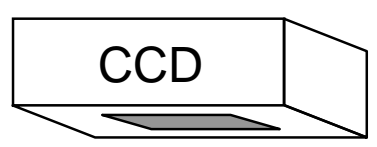

trapped split BECs

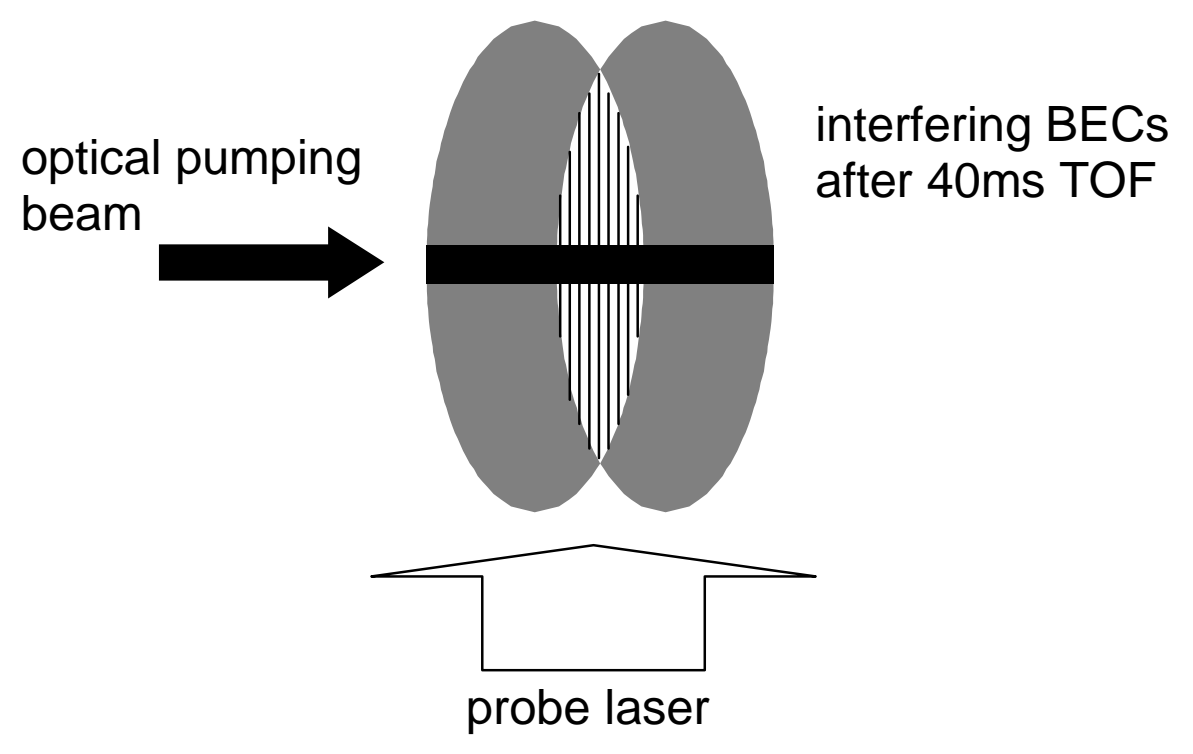




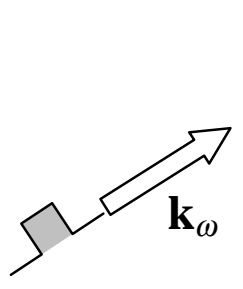

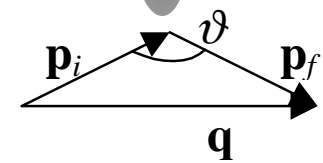

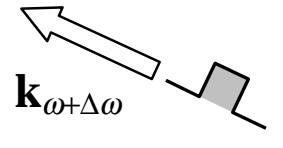

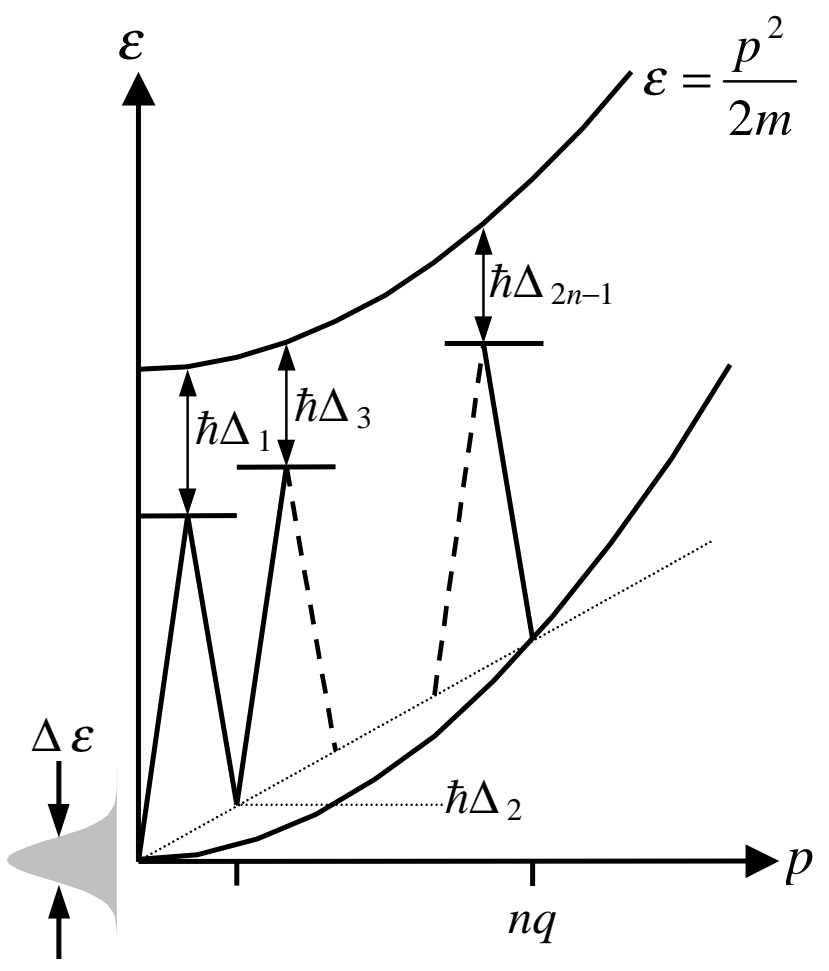




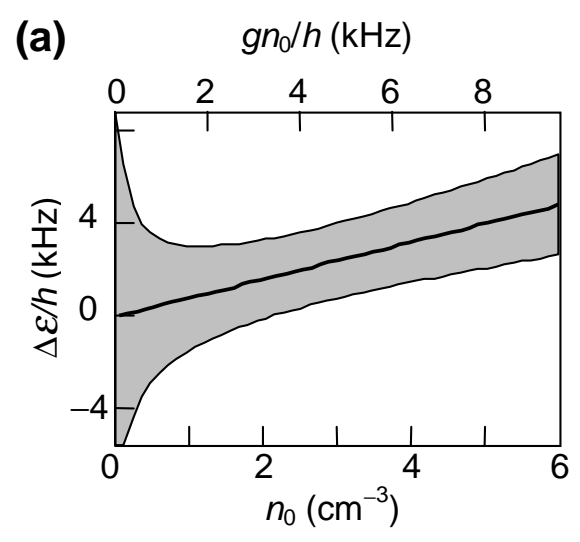

(b)

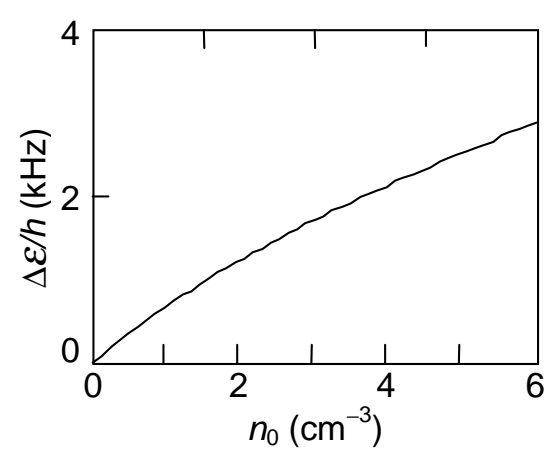

(c)

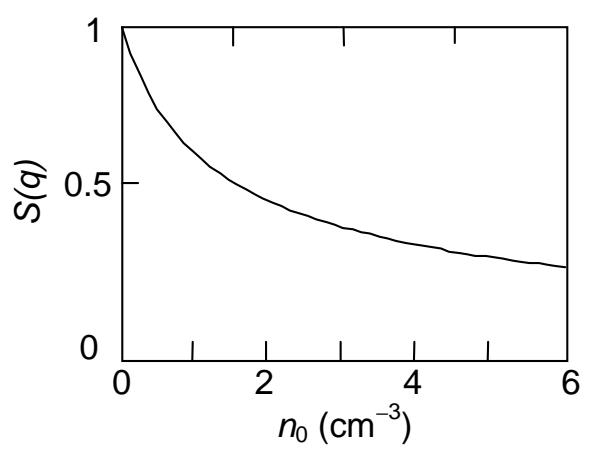


(a)

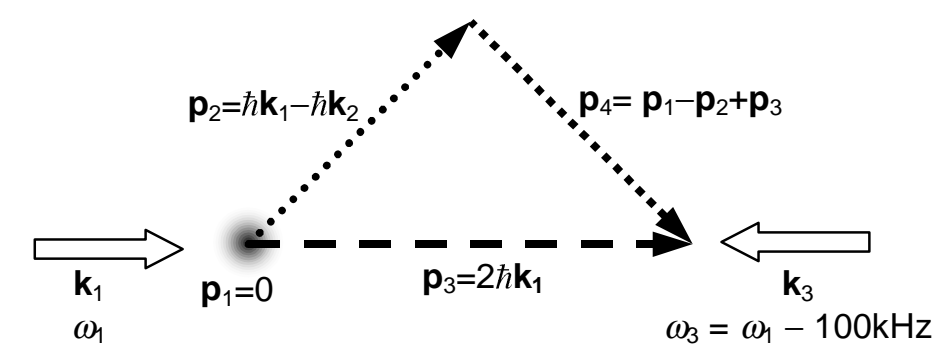

(b)

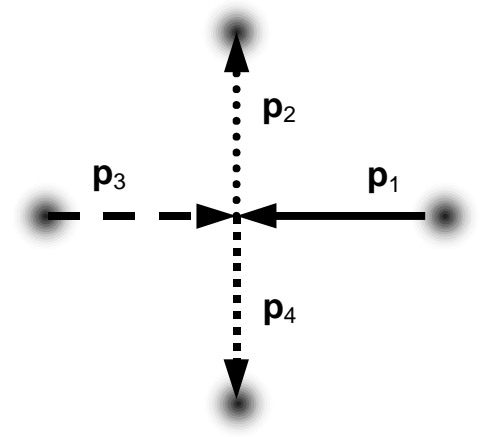

(c)

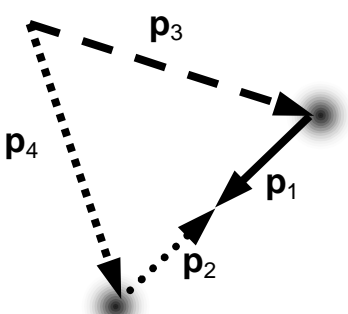




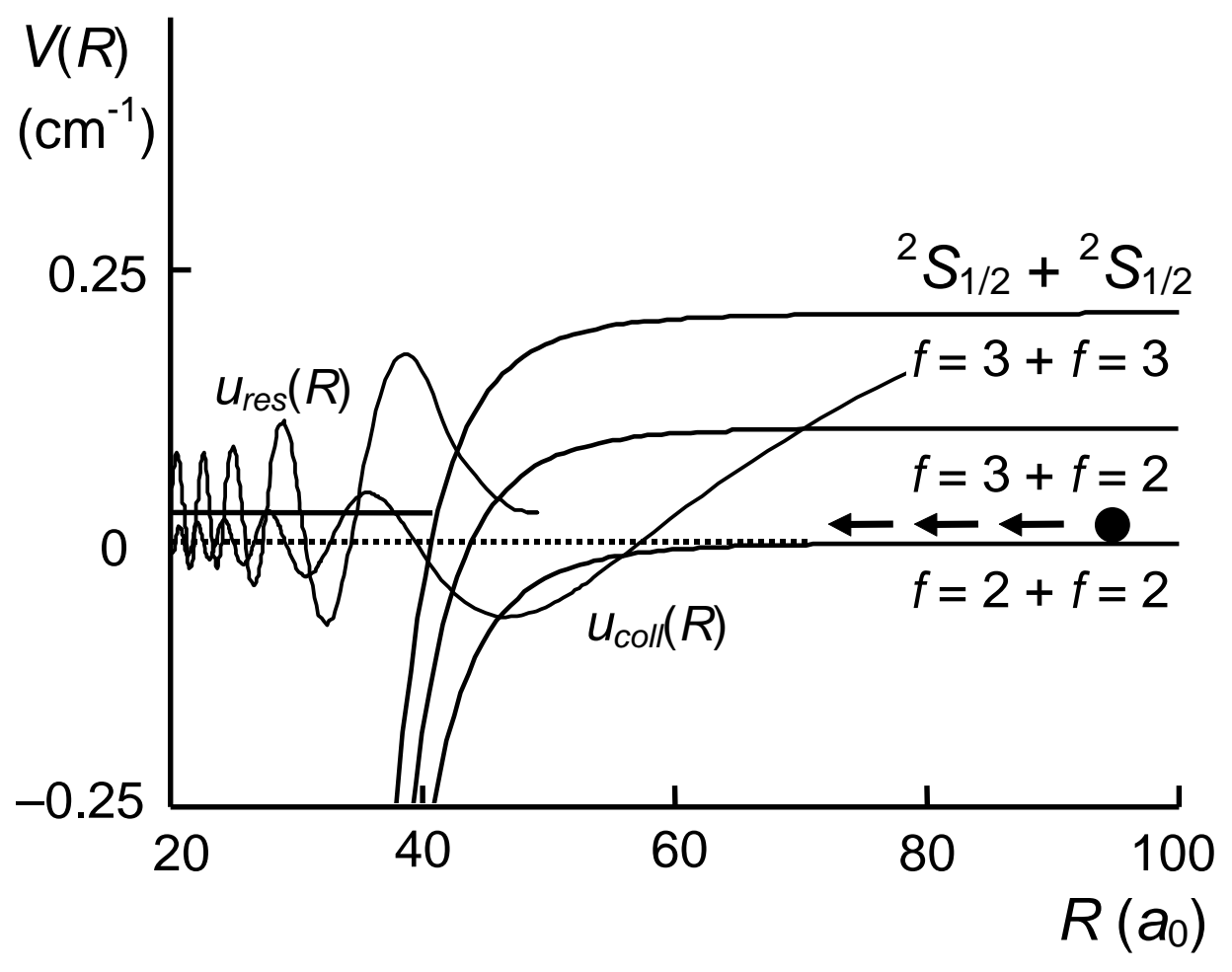




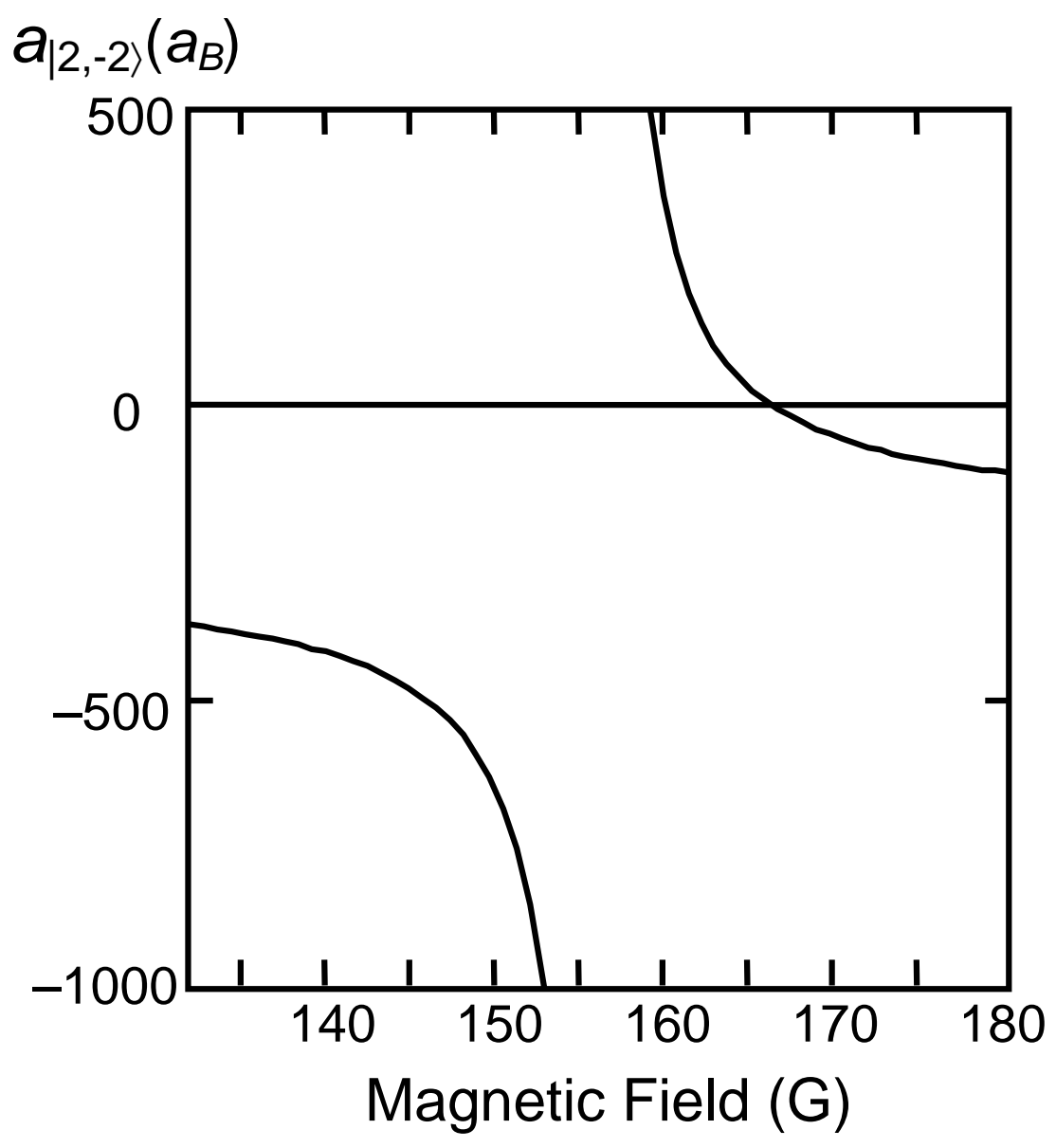




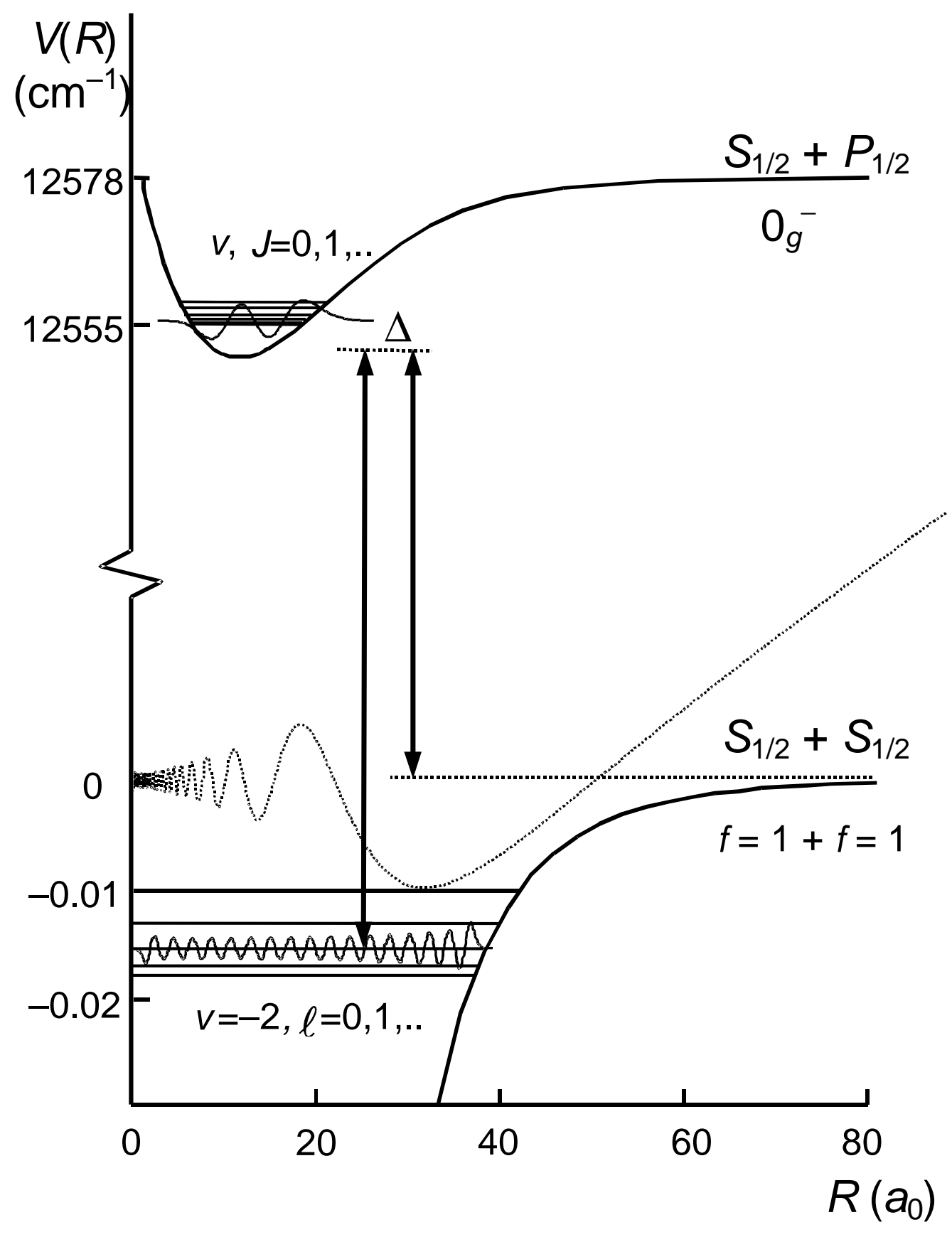

